\title{
Morphogenesis and Genetic Regulation of the Insect Head
}

\author{
Dissertation \\ for the award of the degree \\ "Doctor rerum naturalium" \\ Division of Mathematics and Natural Sciences \\ of the Georg-August-Universität Göttingen \\ within the doctoral program Genes \& Development \\ of the Georg-August University School of Science (GAUSS) \\ submitted by \\ Peter Kitzmann \\ from Lübeck, Germany
}

Göttingen 2016 


\section{Thesis Committee}

Prof. Dr. Gregor Bucher (advisor)

(Johann-Friedrich-Blumenbach Institute of Zoology \& Anthropology; Dpt. of Evolutionary Developmental Genetics)

Prof. Dr. Jörg Großhans

(University Medical Faculty Göttingen; Dpt. of Developmental Biochemistry)

Prof. Dr. Andreas Wodarz

(Institute I of Anatomy Cologne; Dpt. of Molecular Cellbiology)

\section{Members of the Examination Board}

First reviewer: Prof. Dr. Gregor Bucher

(Johann-Friedrich-Blumenbach Institute of Zoology and Anthropology; Dpt. of Evolutionary Developmental Genetics)

Second reviewer: Prof. Dr. Andreas Wodarz

(Institute I of Anatomy Cologne; Dpt. of Molecular Cellbiology)

\section{Further Members of the Examination Board}

Dr. Roland Dosch

(University Medical Faculty Göttingen; Dpt. of Developmental Biochemistry)

Prof. Dr. Ralf Heinrich

(Johann-Friedrich-Blumenbach Institute of Zoology and Anthropology; Dpt. of Cellular Neurobiology)

Prof. Dr. Daniel Jackson

(Courant Research Centre; Dpt. of Geobiology)

\section{Dr. Nico Posnien}

(Johann-Friedrich-Blumenbach Institute of Zoology and Anthropology; Dpt. of Developmental Biology) 


\section{DECLARATION}

I hereby declare that the doctoral thesis entitled,

"Morphogenesis and Genetic Regulation of the Insect Head"

has been written independently and with no other sources and aids than quoted.

Göttingen, May 30 ${ }^{\text {th }}, 2016$

Peter Kitzmann 
Für meine Eltern,

Sabine und Günter Kitzmann 


\section{Danksagung}

An dieser Stelle möchte ich mich bei allen bedanken, die mich auf dem Weg zu dieser Arbeit begleitet und unterstützt haben. Ich bin froh und dankbar für eine sehr schöne Zeit, und glücklich, dass viele dieser Begleiter, falls sie es nicht schon vorher waren, Freunde geworden sind.

Zuallererst möchte ich mich bei Prof. Gregor Bucher bedanken, der es mir überhaupt erst ermöglicht hat an diesem spannenden Projekt zu arbeiten und diese Arbeit zu verfassen. Jedoch hat er mir die Arbeit an dem Projekt nicht nur ermöglicht, sondern mich vor allem auch in jeglicher Hinsicht, zu jeder Zeit aktiv unterstützt. Ich bin sehr dankbar für den wissenschaftlichen Austausch, motivierende Gespräche und unzählige hitzige Diskussionen über Gott und die Welt. Großen Dank und Respekt an einen der besten Betreuer -überhaupt-!

Vielen Dank gilt auch den Mitgliedern meines "Thesis Committees“ Prof. Jörg Großhans und Prof. Andreas Wodarz für das Opfern von Zeit, einen stets unkomplizierten Umgang, aber vor allem für konstruktive Diskussion die diese Arbeit mit geformt haben.

Für das fortwährende Feintuning des Projektes während unzähliger Seminare möchte ich mich auch gerne bei Prof. Ernst A. Wimmer, Dr. Nikola-Michael Prpic-Schäper und Dr. Gerd Vorbrüggen bedanken.

Danken möchte ich auch meinen Kollaborationspartnern in Lyon, Dr. Michalis Averof, Dr. Johannes B. Schinko und vor allem meiner großen Schwester Anna Gilles, für die stets unkomplizierte und konstruktive Zusammenarbeit. Auch meinen Kollaborationspartnern in Dresden, Dr. Pavel Tomancak und Dr. Stefan Münster, möchte ich für die Möglichkeit mit dem Lichtblatt-Mikroskop zu arbeiten danken und für die Aufnahme und das Prozessieren einiger live-imaging Videos.

Vom ganzen Herzen möchte ich mich auch bei Dr. Nico Posnien bedanken, welcher das Projekt in vielen Seminaren, aber auch jederzeit außerhalb dieser durch stetigen Austausch und Diskussionen mit hat reifen lassen. Vor allem möchte ich mich aber auch noch für viele kritische, lustige und ernste Gespräche -egal ob im Stehen, Sitzen oder Joggen- bedanken. Nicos „Koalafications" haben es zu jeder Zeit und auch insbesondere zum Ende der Arbeit immer geschafft mich zu motivieren, nicht zuletzt durch feinste Musiktipps.

Sehr dankbar bin ich auch Dr. Sebastian Kittelmann, Dr. Bernhard G. M. Schmid und Dr. Georg Oberhofer, die alle stets Vorbild waren dazu beigetragen haben, dass ich zu dem Wissenschaftler geworden bin, der ich jetzt bin.

Großer Dank gilt aber vor allem auch Claudia Hinners, die mich jederzeit tatkräftig unterstützt hat und somit den Umfang dieser Arbeit erst mit ermöglicht hat. Danke, liebe Claudia! 
Danken möchte ich auch Elke Küster, die mich nur allzu oft mit Käfern jeglicher Art versorgt hat und für die unermüdliche Pflege der Stocks. Beate Preitz bin ich dankbar für die Einweisung in alle Mikroskope und die Instandhaltung dieser, ohne die ein Großteil dieser Arbeit nicht möglich oder nur erschwert möglich gewesen wäre.

Für die Versorgung mit so einigen Plasmiden und guten Tipps, bin ich Stefan Dippel zu Dank verpflichtet.

Für das Korrekturlesen der Arbeit, möchte ich mich herzlich bei Max Farnsworth bedanken!

Weiterer Dank gilt auch allen HiWis und Studenten an denen ich mich probieren durfte und die mit mir an diversen Projekten gearbeitet haben. Danke für die Mühen und Geduld Julia, Felix und Magdalena! Ihr ward eine große Bereicherung für mich und das Projekt!

Special thanks goes to my best Lab-fellas Georg and Salim, I really enjoyed the time with you and I am happy to call you buddies!

Besonderer Dank gilt auch Hanna, die mich gerade in der heißen Phase der Arbeit, nicht zuletzt mit einem bestechenden Humor, immer wieder motiviert hat und es geschafft hat mich von allem Stress abzulenken! Danke Hanna!

Für eine unnormal gute Stimmung und große Hilfsbereitschaft, möchte ich mich bei allen jetzigen und ehemaligen Mitgliedern des Labors 3 bedanken. Danke, Julia, Janna, Sebastian, Felix, Magdalena, Yonggang, Jürgen, Daniela und Max!

Ich möchte mich bei der ganzen Abteilung für eine super Zeit und fortwährende Unterstützung und Hilfestellung bedanken. Hier geht ein besonders herzlicher Dank an: Christian, Jonas, Beni, Christoph, Natascha, Jana und Kolja.

Dankbar bin ich auch dem ganzen TA-Team, Claudia Hinners, Katrin Kanbach, Helma Griess, Angelika Löffers und Bettina Hucke, die den Laden die ganze Zeit im Hintergrund am Laufen gehalten haben und mir sehr viel Arbeit erspart haben. Ich möchte mich auch herzlich bei den Damen des Sekretariats, Birgit Rossi, Bettina Hucke, Merle Eggers, Selen Pfändner, Inga Schild und Constanze Gerhards bedanken, die es immer unkompliziert geschafft haben jeden komplizierten bürokratischen Kram zu erledigen und immer für ein netten Schnack zu haben waren.

Zu guter Letzt möchte ich mich bei all jenen bedanken, bei denen ich mich glücklich schätzen darf sie Freunde zu nennen. Euch namentlich zu erwähnen würde den Rahmen dieser Arbeit sprengen. Aber Ihr wisst von wem ich spreche. Ihr seid ja alle Füchse! Ich bin Euch allen mega dankbar, da Ihr mich alle stets auf Eure Art unterstützt, bespaßt und, wenn nötig, mit allen Mitteln abgelenkt habt. 


\section{Contents}

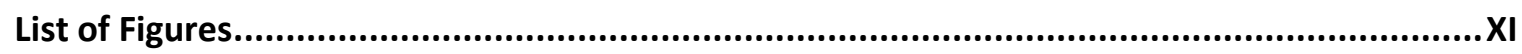

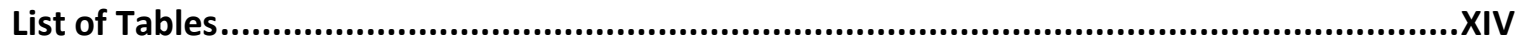

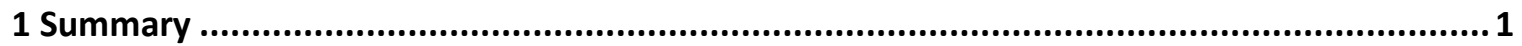

2 Introduction

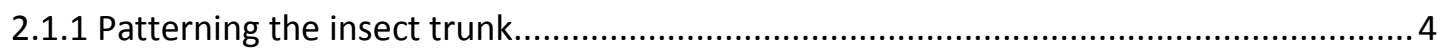

2.1.2 Genetic regulation of insect head development..........................................................

2.1.3 Tribolium - a model organism for insect head development .........................................

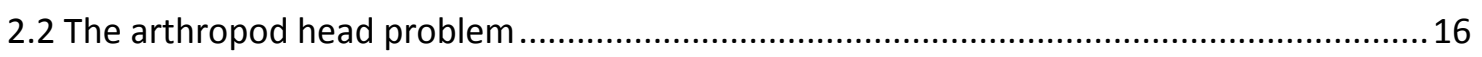

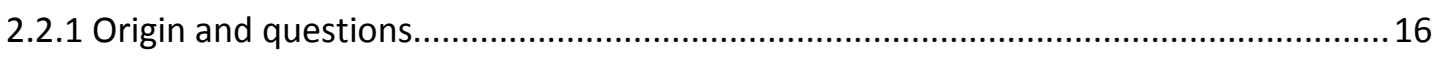

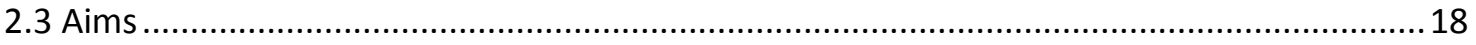

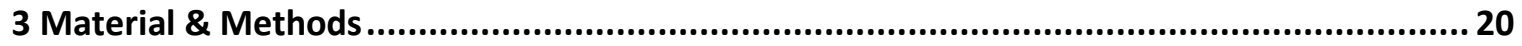

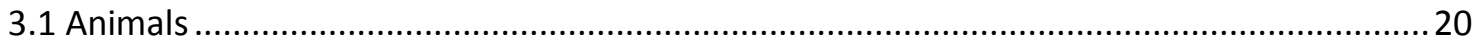

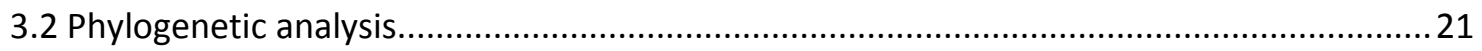

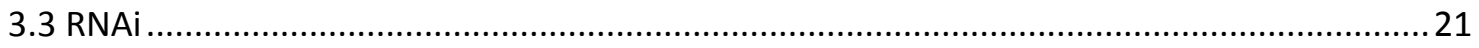

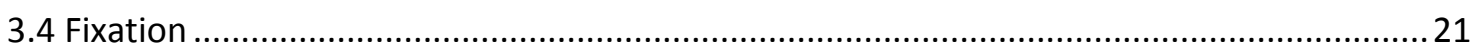

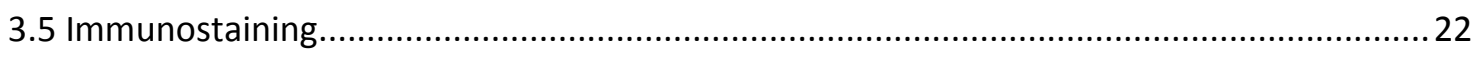

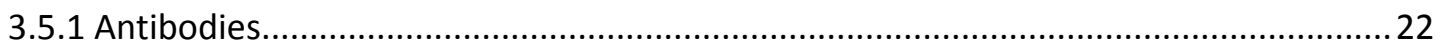

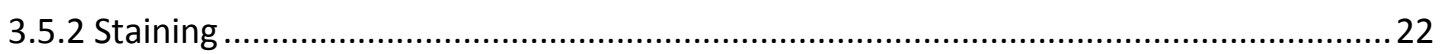

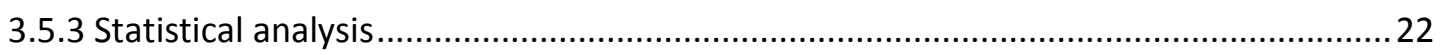

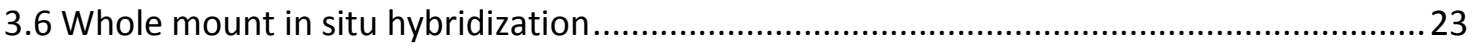

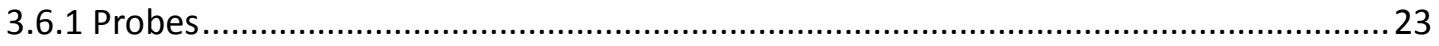

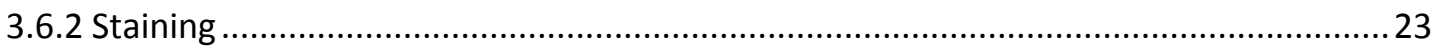

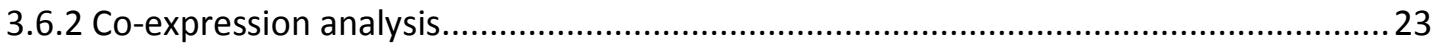

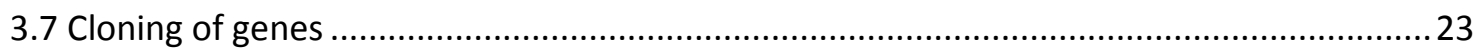

3.8 Generation of H2Av::EGFP and H2Av::C3PA-GFP chimeric reporter proteins ......................2. 24

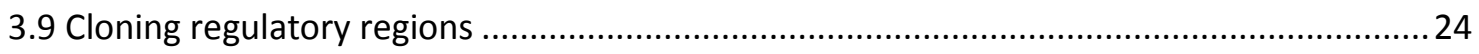

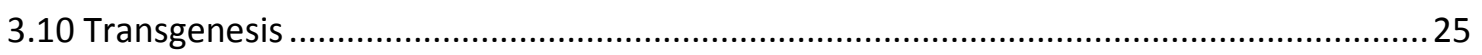

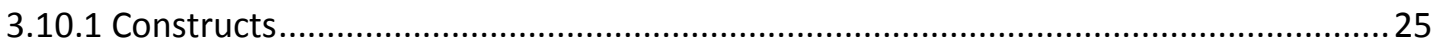

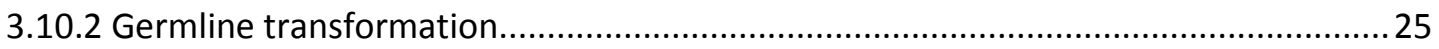

3.10.3 Transformation marker and marker detection ..........................................................25

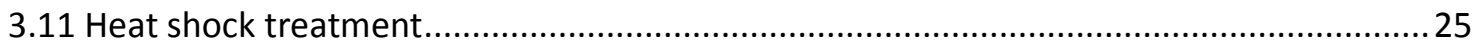


3.12 Photoactivation

3.13 Image documentation and processing............................................................ 26

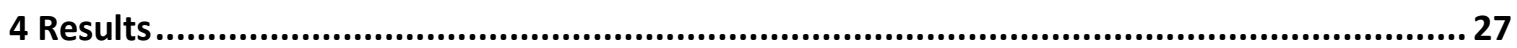

4.1 Tc-foxq2 - a novel player in anterior head development of Tribolium .............................. 27

4.1.1 iB_03837 targets the Tribolium ortholog of Foxq2 ............................................... 27

4.1.2 Tc-foxq2 knock-down phenotype in ectodermal tissue........................................ 28

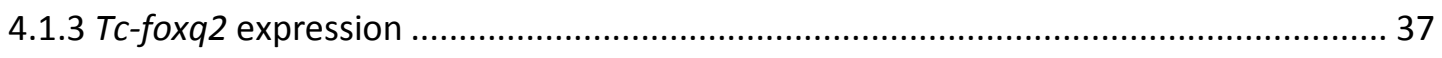

4.1.4 Uncovering the role of Tc-foxq2 within the gene regulatory network of the anterior pre-segmental region........................................................................................... 42

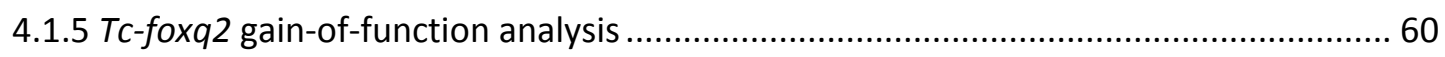

4.1.6 Tc-foxq2 is required for proper brain formation ...................................................... 72

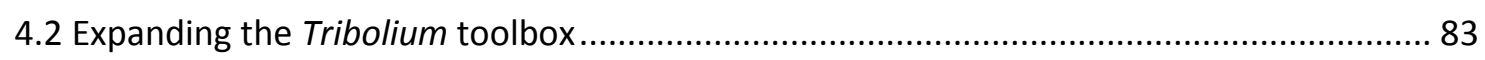

4.2.1 Generating transgenic lines driving strong and ubiquitous expression of a nuclear

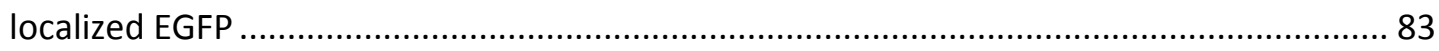

4.2.2 Generation of cell marking lines for tracking experiments to assemble an exact head

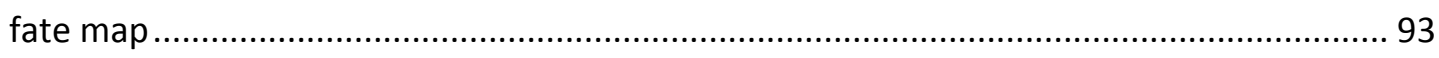

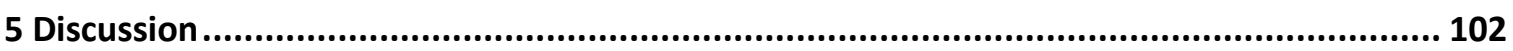

5.1 Tc-foxq2 is required for head and brain development ............................................ 102

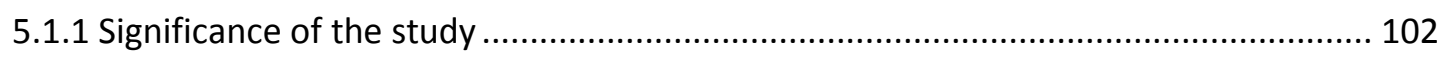

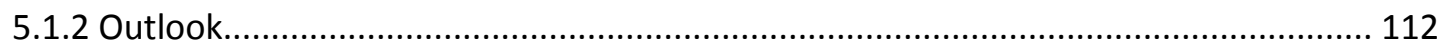

5.2 New tools to study morphogenetic movements ................................................. 117

5.2.1 The transgenic C3PA-GFP photoactivation lines are a powerful tool for cell marking and fate mapping ............................................................................................ 117

5.2.2 The genetic Cre/loxP cell marking system is a powerful tool, but has to be improved

5.2.3 The $\alpha$ Tub1/rps3/PUb promoters are ubiquitously active at all embryonic stages ..... 119

5.2.4 Utilization of the new imaging lines...................................................... 120

5.3 The arthropod head problem ........................................................................... 122

5.3.1 Tc-foxq2 - Implementations for the arthropod head problem ............................... 122

5.3.2 Imaging lines and implementations for the arthropod head problem.................... 122

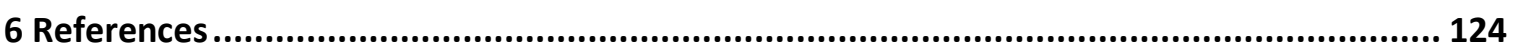

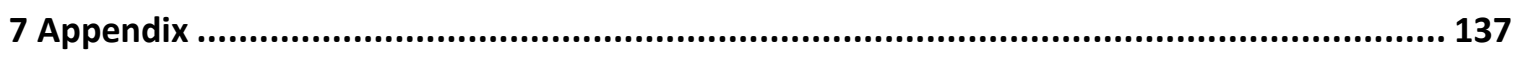

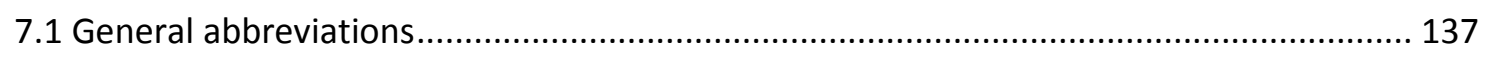

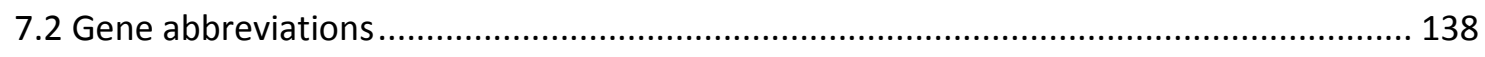

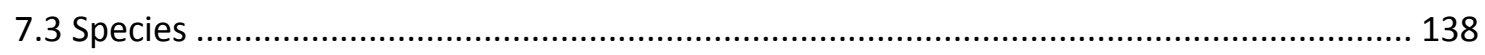


7.4 Supplementary tables, figures, sequences, and videos

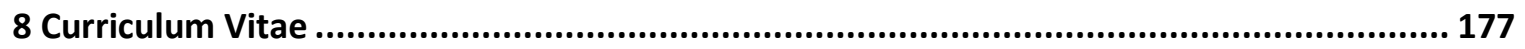




\section{List of Figures}

2.1 Gene regulatory network of the anteriormost region in Drosophila. 6

2.2 Composition and patterning of the Tribolium head. $\quad 7$

2.3 Gene regulatory network of the anteriormost head region in Tribolium. 10

2.4 Structure of a classical Fox protein domain (FoxQ1). 12

2.5 foxq2 gene subfamily members are found in almost all phyla of the metazoan kingdom. $\quad 14$

2.6 Conservation of an apical patterning gene set in different species across metazoan species. 16

$\begin{array}{ll}2.7 \text { Classical head fate map hypothesis. } & 17\end{array}$

$\begin{array}{ll}2.8 \text { The bend and zipper model predicts a new head fate map. } & 17\end{array}$

4.1 Phylogenetic tree of Foxq2 proteins within the Metazoa. 28

4.2 Qualitative analysis of Tc-foxq2 pRNAi reveals a labrum-specific phenotype in L1 larvae. $\quad 29$

4.3 Quantitative analysis of the $T c-f o x q 2^{\text {pRNAi }}$ epidermal L1 defects confirms the phenotype and $\begin{array}{ll}\text { excludes off-target effects. } & 30\end{array}$

4.4 Quantitative analysis of the $T c-f o x q 2^{\mathrm{pRNAi}}$ epidermal L1 phenotypes in two different strains shows no considerable strain specific effects.

4.5 Qualitative analysis of the embryonic $T c-f o x q 2^{\text {pRNAi }}$ phenotype and its correlation with cell death rates.

4.6.1 Overview of the location of the most relevant embryonic head structures for this study. 38

4.6 Tc-foxq2 is expressed in a highly dynamic pattern at the anterior pole. 39

4.7 Endogenous Tc-foxq2 mRNA is not completely abolished after Tc-foxq2 pRNAi. 41

4.8 Co-expression analyses of Tc-foxq2 and anterior head patterning genes I.

4.9 Co-expression analyses of $T c$-foxq2 and anterior head patterning genes II. 46

4.10Tc-foxq2 ${ }^{\text {pRNAi }}$ embryos show reduced Tc-six3 expression domains. $\quad 48$

4.11 Tc-foxq2 $2^{\text {PRNAi }}$ embryos show reduced Tc-cnc and Tc-croc expression domains. 49

4.12 Tc-foxq2 ${ }^{\text {pRNAi }}$ embryos show slightly altered Tc-scro and Tc-fkh expression profiles. $\quad 50$

4.13 Tc-foxq $2^{\text {pRNAi }}$ embryos show reduced $T c-c h x$ and $T c-s i x 4$ expression domains. 51

4.14 Tc-foxq2 ${ }^{\text {pRNAi }}$ embryos show reduced Tc-rx expression domains. 52 
4.15 Tc-foxq $2^{\text {pRNAi }}$ embryos show a reduction of the labral $T c-w g$ expression domains.

4.16 $T c-s i x 3^{\mathrm{pRNAi}}$ and $T c-$ croc $^{\mathrm{pRNAi}}$ embryos show altered $T c$-foxq2 expression profiles.

4.17Tc-arr ${ }^{\text {pRNAi }}$ and $T c-m i b 1^{\text {pRNAi }}$ embryos show altered $T c$-foxq2 expression profiles.

4.18 $T c-s c r O^{\text {pRNAi }}$ and $T c-c n c^{\text {pRNAi }}$ lead to a changed $T c$-foxq2 expression profile at late stages only.

4.19 $T c$-six $4^{\text {pRNAi }}$ and $T c$-ch $x^{\text {pRNAi }}$ embryos show an altered $T c$-foxq 2 expression profile at late stages.

4.20 $T c-f k h^{\text {pRNAi }}$ embryos show a secondary alteration of $T c-f o x q 2$ expression, whereas $T c-r x^{\text {pRNAi }}$ embryos show no considerable change in $T c$-foxq 2 expression profile.

4.21 Transgenesis construct for heat shock-inducible $T c$-foxq2 misexpression lines.

4.22 Tc-foxq2 gain-of-function lines show heat shock-induced ectopic Tc-foxq2 expression.

4.23 Embryonic Tc-foxq2 gain-of-function results in defects in L1 larval cuticles.

4.24 Larval epidermal defects after different onsets of embryonic Tc-foxq2 gain-of-function.

4.25 Ectopic Tc-foxq2 expression leads to an increased number of apoptotic cells.

4.26 Ectopic $T c$-foxq2 expression impacts head patterning gene expression profiles (strong effects).

4.27 Ectopic Tc-foxq2 expression impacts head patterning gene expression profiles (mild effects).

4.28 Embryonic knock-down of Tc-foxq2 function leads to defects in L1 larval brains.

4.29 Embryonic Tc-foxq2 knock-down leads to mushroom body defects in L1 larvae.

4.30 Embryonic gain of $T c$-foxq2 function leads to weak neural defects in L1 larvae.

4.31 Embryonic gain of Tc-foxq2 function leads to affected mushroom bodies in L1 larvae. $\quad 80$

4.32 Analysis of cell death rates within the neurogenic head region in $T c-f o x q 2^{\text {pRNAi }}$ embryos.

4.33 Qualitative comparison of signal intensities and localizations of three different ubiquitous nuclear reporter lines at early embryonic stages.

4.34 Qualitative comparisons of signal intensities of three different ubiquitous nuclear reporter lines at larval and pupal stages.

4.35 Qualitative comparison of signal intensity and localization of three different ubiquitous nuclear reporter lines in ovaries. 
4.37 Early embryonic development imaged using the transgenic $\alpha$ Tubulin1P-H2Av::EGFP line in combination with LSFM technique I.

4.38 Early embryonic development imaged with the transgenic $\alpha$ Tubulin1P-H2Av::EGFP line in combination LSFM technique II.

4.39 Comparison between conventional LSM and LSFM imaging of the anterior embryonic cap.

4.40 Test of C3PA photoactivation in different transgenic lines.

4.41 Test of C3PA photoactivation capacities at larval and pupal stages. 96

4.42 Scheme of the genetic cell marking system exploiting the Cre/loxP system. 98

4.43 Test of the genetic cell marking system. 100

5.1 Tc-foxq2 is an upstream player within the gene regulatory network of the anterior Tribolium head.

5.2 Late effects of and on Tc-foxq2. 105

5.3 Conserved expression pattern of foxq2 in metazoan kingdom-spanning species. 106

5.4 Co-expression of foxq2/six3 at different developmental stages of different Metazoa. 109

S7.15 pB[3xP3-gTc'v;Tc' $\alpha$ Tub1P-Tc'H2Av::EGFP].

S7.17 pB[3xP3-gTc'v;Tc'rpS3P-Tc'H2Av::EGFP].

S7.19 pB[3xP3-gTc'v;Tc'PUbP-Tc'H2Av::EGFP]. 156

S7.21 pB[3xP3-gTc'v;Tc' $\alpha$ Tub1P-C3PA-GFP]. 160

S7.23 pB[3xP3-gTc'v;Tc' $\alpha$ Tub1P-Tc'H2Av:: C3PA-GFP]. 164

S7.25 pB[3xP3-gTc'v;Tc'hsp68-Tc'foxq2]. 168

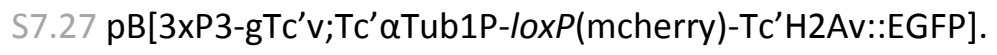




\section{List of Tables}

S7.1 Tc-foxq2 $2^{\text {RNAi_a }}$ general cuticle phenotype using $1 \mu \mathrm{g} / \mu \mathrm{l}$ dsRNA in SB. 139

S7.2 Tc-foxq $2^{\text {RNAi_a }}$ head defects using $1 \mu \mathrm{g} / \mu \mathrm{l}$ dsRNA in SB. $\quad 139$

S7.3 Tc-foxq $2^{\text {RNAi_b }}$ general cuticle phenotype using $1 \mu \mathrm{g} / \mu \mathrm{l}$ dsRNA in SB. 139

S7.4 Tc-foxq $2^{\text {RNAi_b }}$ head defects using $1 \mu \mathrm{g} / \mu \mathrm{l}$ dsRNA in SB. $\quad 140$

S7.5 Tc-foxq $2^{\text {RNAi_b }}$ general cuticle phenotype using $1 \mu \mathrm{g} / \mu \mathrm{l}$ dsRNA in pBa19 $\times$ black. $\quad 140$

S7.6 Tc-foxq $2^{\text {RNAi_b }}$ head defects using $1 \mu \mathrm{g} / \mu \mathrm{l}$ dsRNA in pBa19 $\times$ black. $\quad 140$

S7.7 Number (pre-normalization) of apoptotic cells per untreated SB embryo. $\quad 140$

S7.8 Number (pre-normalization) of apoptotic cells per Tc-foxq2 $2^{\text {pRNAi }}$ embryo. 142

S7.9 General cuticle phenotype after embryonic Tc-foxq2 gain-of-function (HS: 9-13 h AEL). 145

S7.10 Cuticle defects (\%) after embryonic Tc-foxq2 gain-of-function (HS: 9-13 h AEL). $\quad 145$

S7.11 General cuticle phenotype after embryonic Tc-foxq2 gain-of-function (HS: 14-20 h AEL). 146

S7.12 Cuticle defects (\%) after embryonic Tc-foxq2 gain-of-function (HS: 14-20 h AEL). $\quad 146$

S7.13 General cuticle phenotype after embryonic Tc-foxq2 gain-of-function (HS: 20-25 h AEL). 146

S7.14 Cuticle defects (\%) after embryonic Tc-foxq2 gain-of-function (HS: 20-25 h AEL). $\quad 147$ 



\section{1}

\section{Summary}

Natural selection and the struggle for ecological niches were the driving force for the origin of a tremendous number of animal groups with different body shapes. The segmental organization of insects enhanced their evolutionary specialization. Adaptations led to different morphologies, for instance with respect to the head. The head, which carries the feeding apparatus and the main sensory centers enabling interaction with the environment, is one prerequisite for evolutionary success. The segmental structure of the insect head facilitated evolution of adaptations with respect to morphology. However, although the head is of great importance for the evolutionary success, there is no comprehensive understanding of the gene network regulating anterior head development until now. Furthermore, the genetic basis for the different insect head morphologies is unknown. Moreover, there are open questions concerning the segmental structure of the head and how morphogenetic movements lead to the adult head.

During the last years the red flour beetle Tribolium castaneum was developed as a major model organism for studying insect head development. Recent studies were able to successively uncover the genetic interactions of anterior head development. However, so far the gene set and its interaction are not comprehensively unraveled. $T c$-foxq 2 is a novel regulator of head development identified with a highly specific head phenotype in the iBeetle screen. Hence, I studied its potential function in the anterior head gene regulatory network. Another open question is the contribution of the different embryonic head regions to the adult head. To provide new insights regarding this question I wanted to provide new features for the Tribolium toolbox.

In this study I was able to show that $T c-f o x q 2$ is specifically expressed in the anterior presegmental head region, similar to the conserved expression pattern, which is located at the anterior pole in metazoan species. Further, I was able to show that $T c$-foxq2 is an upstream player within the anterior head gene regulatory network, forming a conserved patterning unit together with $T c$-six3. $T c$-foxq2 knock-down results in an increased cell death rate within the clypeo-labral region, which consequently leads to reduced labral buds at embryonic stages. This 
defect is also reflected in L1 larval cuticles showing a labrum that is strongly reduced or completely absent. Further, using neurogenic in vivo imaging reporter lines I was able to show that embryonic knock-down of $T c$-foxq2 function leads to central body and mushroom body defects. Moreover, I was able to generate new reporter lines that drive expression of the chimeric H2Av::EGFP nuclear marker protein under control of the Tc- $\alpha$ Tubulin1 promoter or the Tc-ribosomal protein subunit3 promoter. I analyzed the lines with respect to localization of the marker protein, signal distribution within the embryo, signal intensity in different developmental stages and tissues, and viability of the different transgenic lines. I could show that the lines are functional and that the Tc- $\alpha$ Tubulin1 promoter line is best suited for being analyzed with lightsheet imaging. Finally, I was able to generate and to show functionality of new in vivo imaging lines for laser-induced cell marking and genetic cell marking. 


\section{2}

\section{Introduction}

\subsection{Patterning the insect body}

\subsubsection{Patterning the insect trunk}

With more than a million described species, insects are the most diverse and species-rich animal class (Chapman et al., 2009; Grimaldi and Engel, 2005). The modular segmental body plan was the prerequisite for dramatic radiations and the evolutionary success (Chipman, 2010; Tautz et al., 1994). The genetic basis for the development of a segmented body plan was studied for decades in the model organism Drosophila melanogaster (Drosophila). Thus, anteroposterior (AP) patterning is well understood and best known in Drosophila (Pick, 1998). It has been shown that a multi level hierarchical gene cascade is responsible for building the segments along the AP-axis. This cascade involves in the first place maternal factors, which set up the anterior and posterior system by localized mRNAs like bicoid (bcd; anterior) and nanos (nos; posterior), as well as the terminal and dorso-ventral systems involving signaling pathways like torso (tor; terminal) and decapentaplegic (dpp; dorsal). These systems determine the primary body axes and the terminal regions of the embryo. The body is further subdivided in to smaller regions by the expression of gap genes, which are regulated by the maternal system. These regions are then subdivided further by the pair-rule gene expression regulated by gap genes. In turn, pairrule genes regulate the expression of segment polarity genes, which determine segment boundaries and establish polarity in each segment. Eventually, all these levels are regulating the expression of homeotic selector (Hox) genes, which are required for segment identity specification (Akam, 1987; Cohen and Jürgens, 1991; Ingham, 1988; Johnston and NüssleinVolhard, 1992).

The insect head is of high importance due to its roles in sensory input integration, generation of adequate behavioral output, and feeding. But, in contrast to AP patterning, the genetic basis of head patterning is currently not understood comprehensively in any insect. However, due to the 
fact that neither Hox genes nor pair-rule genes are expressed at the anteriormost region, this region has to rely on a completely different gene regulatory network (Bucher and Wimmer, 2005; Posnien et al., 2010).

\subsubsection{Genetic regulation of insect head development}

\subsubsection{Head patterning in Drosophila}

The subsequent description is mainly referring to (Rogers and Kaufman, 1997). In Drosophila, it has been shown that the posterior part of the head, built by the segments bearing the gnathal appendages, is patterned like the trunk segments via the classical patterning cascade (Akam, 1987; Cohen and Jürgens, 1991; Ingham, 1988; Johnston and Nüsslein-Volhard, 1992).

However, the segmental part of the anterior head composed of the intercalary segment and the antennal segment is patterned in a different way. The maternal contribution in anterior head development is the same. The anterior maternal morphogen $b c d$, terminal factors like tor, and dorso-ventral factors like dorsal $(d l)$ are required for activating genes of the second level of hierarchy. The maternal factors are regulating head gap-like genes orthodenticle, empty spiracles, buttonhead, and sloppy paired, which are expressed in broad overlapping domains in the anterior head (Cohen and Jürgens, 1991, 1990; Dalton et al., 1989; Finkelstein et al., 1990; Grossniklaus et al., 1992; Mohler, 1995; Walldorf and Gehring, 1992; Wimmer et al., 1997, 1993). In contrast to the posterior head, there are no pair-rule genes expressed in the anterior head and consequently not involved in the development of this region. Instead, head gap-like genes are either directly acting on segment polarity genes, like engrailed, hedgehog, and wingless (Gallitano-Mendel and Finkelstein, 1998; Mohler, 1995; Rogers and Kaufman, 1997) or indirectly by regulation of second level regulators like collier (col; Crozatier et al., 1996) or segment identity genes like cap'n'collar (cnc; Crozatier et al., 1999).

\subsubsection{Anteriormost head patterning in Drosophila}

The subsequent description is mainly referring to Rogers and Kaufman, 1997. The anteriormost region of the head, which is composed of the clypeolabral, foregut, stomodeal, and endodermal anlagen (Rogers and Kaufman, 1997), depends on a very different gene regulatory network. The anteriormost region is patterned by the anterior, terminal, and dorsal maternal system 
(Grossniklaus et al., 1994). In this region the maternal factors bcd and torso are required for regulation of the terminal gap genes tailless $(t / l)$ and huckebein $(h k b)$. In turn, $t / l$ and $h k b$ regulate the transcription factors and second order regulators $c n c$ and crocodile (croc), which are involved in the formation of the clypeolabrum, forkhead $(\mathrm{fkh})$, which is required for foregut formation, and serpent (srp), which is necessary for proper formation of the anterior midgut (Figure 2.1; Brönner et al., 1994; Bronner and Jackle, 1991; Brönner and Jäckle, 1996; Häcker et al., 1995; Mohler, 1993; Reuter and Leptin, 1994; Rogers and Kaufman, 1997; Weigel et al., 1989). The transcription factor sine oculis homeobox homolog 3/optix (six3/optix) (six3) is required for the formation of clypeolabrum as well, but its position in the gene regulatory network has not been studied so far (Coiffier et al., 2007). However, due to several limitations (see next chapter), there is no comprehensive understanding about patterning of the anteriormost head region in Drosophila. Therefore, in order to get a comprehensive knowledge of the anterior head gene regulatory network another organism has to be exploited. Only comprehensive understanding of the gene regulatory networks can provide information about the basis of evolution and diversification of head morphologies.

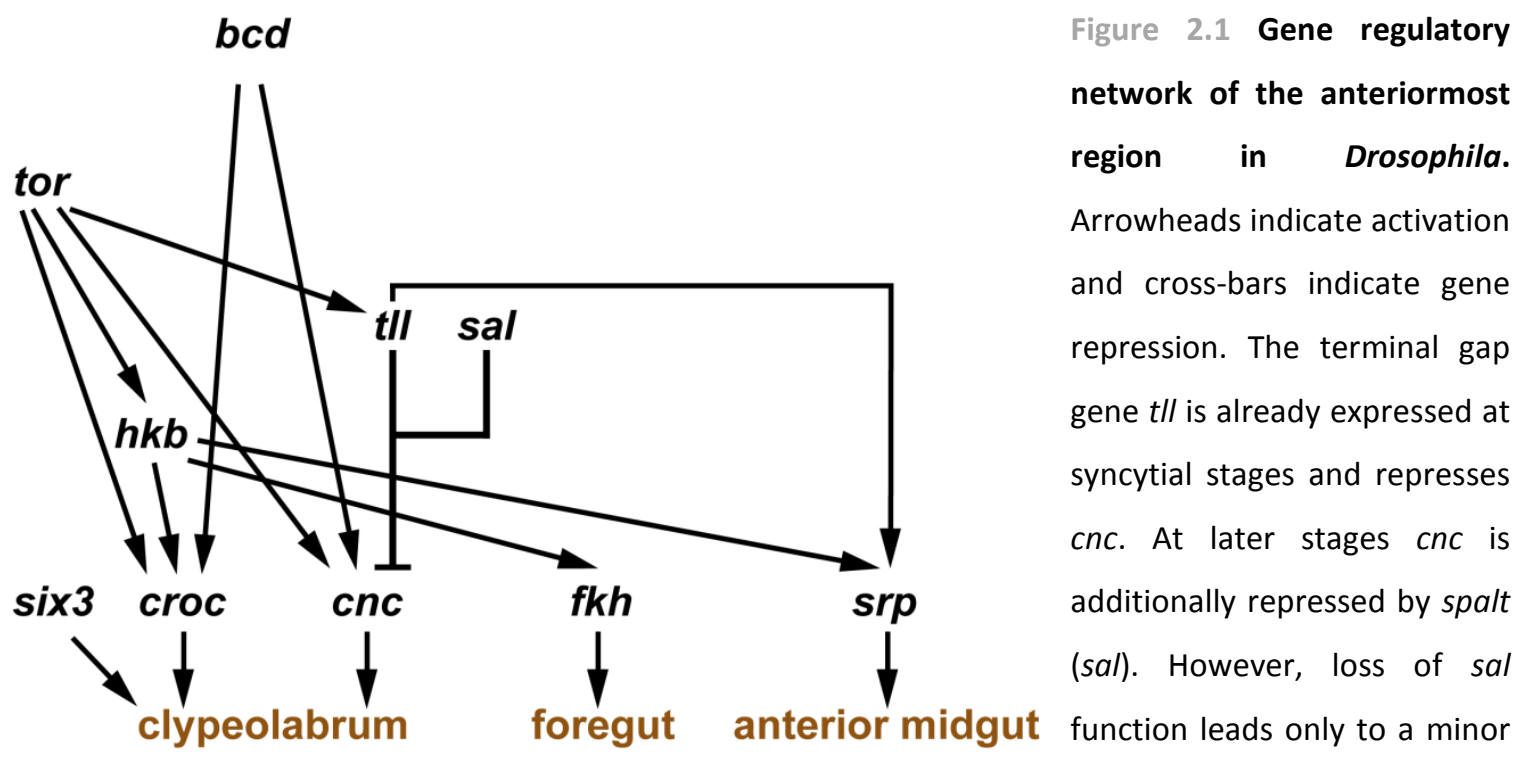

alteration of cnc expression. six 3 was shown to be required for clypeolabral patterning but interaction with other genes has not been studied. (Based on Coiffier et al., 2007; Rogers and Kaufman, 1997) 


\subsubsection{Tribolium - a model organism for insect head development}

Drosophila is, for plenty of reasons, the prime model organism for studying insect development (Bolker, 2012; Kohler, 1994; St Johnston, 2002). However, the suitability for studying insecttypical head development is limited due to several reasons. First, Drosophila develops as a longgerm insect (Davis and Patel, 2002). Thus, the head anlagen are located at the anterior pole of the egg and depend on anterior, terminal, and dorsal signaling, while most insects show head anlagen located in the ventral median region at blastoderm stages (Sander 1976). Second, the Drosophila larval head is turned from outside to the inside during embryonic development, in a process called head involution (Grossniklaus et al., 1994). This leads to a highly derived head morphology and hampers the phenotypic analysis due to lack of morphological markers. Further, developmental defects due to mutations often interfere with head involution, thus causing additional secondary defects (Merrill et al., 1989; Posnien et al., 2010; Rogers and Kaufman, 1997).

I used the red flour beetle Tribolium castaneum (Tribolium; HERBST 1797) as model organism for insect-typical head development for several reasons (Bucher and Wimmer, 2005; Klingler, 2004). Tribolium is a representative of the most diverse and species-rich order across the tree of life, i.e. the coleopterans (beetles; Beutel, 2000; Grimaldi and Engel, 2005) and a cosmopolitan pest of stored grain (Klingler, 2004; Sokoloff, 1974; Zettler, 1991). Tribolium passes through embryonic development in the short germ-mode, in which posterior segments are progressively added from a posterior growth zone, reflecting a more insect-typical mode of development (Davis and Patel, 2002; Klingler, 2004; Lynch and Desplan, 2003; Tautz et al., 1994). At early embryonic stages the head is located at a ventral sub-terminal position, thus depending on ventral signaling as well as signaling from the more anterior extra-embryonic tissue (Posnien et al., 2010). Furthermore, larval stages show a fully everted head, carrying all typical appendages and a highly specific head bristle pattern, which provides landmarks for phenotypic analysis (Chapman, 1982; Posnien et al., 2010; Schinko et al., 2008). Moreover, a large versatile toolbox for studying insect development has been set up during the last two decades. The genome of Tribolium is fully annotated, serving as basis for many different questions and experiments (Richards et al., 2008). Further, the Tribolium toolbox provides several techniques for genetic manipulation, e.g. robust and systemic RNA interference (RNAi) feasible for all developmental stages (Brown et al., 1999; Bucher et al., 2002; Tomoyasu et al., 2008; Tomoyasu and Denell, 2004), spatio-temporal control of RNAi (J. Ulrich, unpublished), heat shock-based gene misexpression (Schinko et al., 2012), and GAL4/UAS-based misexpression (Schinko et al., 2010). Transposon-mediated transgenesis (Berghammer et al., 1999), as well as CRISPR (clustered 
regularly interspaced short palindromic repeats) -mediated transgenesis has been established (Gilles et al., 2015; Gilles and Averof, 2014). Further, powerful in vivo imaging tools were generated, e.g. a nuclear reporter line (El-Sherif et al., 2012; Sarrazin et al., 2012), embryonic mRNA injection of reporter molecules (Benton et al., 2013), and protocols for light-sheet-based imaging and data processing (Strobl et al., 2015; Strobl and Stelzer, 2014). Moreover, the largescale and unbiased 'iBeetle' RNAi screen can be used to find new genes required for a process apart from the classical candidate gene approach (Schmitt-Engel et al., 2015). Finally, the transposon-based mutagenesis screen 'GEKU' provided numerous transgenic enhancer trap and embryonic lethal mutant lines (Trauner et al., 2009).

\subsubsection{Drosophila gene function is not conserved well in Tribolium}

With respect to the genetic regulation of head development in Tribolium it appears that the mechanisms for patterning the gnathocephalic part of the head are similar to Drosophila, especially concerning downstream levels, like pair-rule genes and segment polarity genes (Brown et al., 1994; Choe et al., 2006; Choe and Brown, 2009, 2007; Farzana and Brown, 2008; Oppenheimer et al., 1999; Peel et al., 2013; Schaeper et al., 2010). However, there are some major differences considering the upstream regulators of head development in Tribolium, reflecting the more ancestral and insect-typical regulation of development (Bucher and Wimmer, 2005; Klingler, 2004; Schröder et al., 2008). It was predicted that early patterning of the anterior head region must be very different in Tribolium compared to Drosophila, because of the different positions of the head anlagen in Drosophila (Posnien et al., 2010). For instance, tor is present but plays no role in head development (Schoppmeier and Schröder, 2005), whereas bcd is not existent in Tribolium (Brown et al., 2001; Stauber et al., 1999), instead Tc-axin (Fu et al., 2012) and Tc-mex3 (Schoppmeier et al., 2009) are required for specification of the head region. Regarding the anterior head it has been shown that the head gap-like genes diverged significantly in their function (Kittelmann et al., 2013; Schinko et al., 2008). The head patterning function of Tc-orthodenticle is conserved specifically during later stages. However, the early regionalization function is required for all segments formed at the blastoderm stage. Further, Tc-sloppy-paired affects only the head vertex in the procephalic region (Posnien et al., 2011b). Tc-empty spiracles shows a only partial loss of one segment upon deletion and Tc-buttonhead has no considerable function at all (Schinko et al., 2008). In turn, Tc-knirps (Tc-kni) is required for formation of the antennal and mandibular segments, but has no function in Drosophila head development (Cerny et al., 2008). Further, Tc-kni expression is regulated by the pair-rule gene 
engrailed indicating an evolutionary difference in the classical gene hierarchy (Peel et al., 2013). However, more downstream acting genes that are involved in differentiation seem to be conserved, e.g. Tc-cnc, Tc-croc, and Tc-fkh (Economou and Telford, 2009; Kittelmann et al., 2013;

\subsubsection{Anteriormost head patterning in Tribolium}

Recent studies tried to elucidate the gene regulatory network of the anteriormost pre-segmental head region and found out that this region is patterned by a unique gene regulatory network, which is independent of Hox genes and pair-rule patterning (Figure 2.2B, Kittelmann et al., 2013; Posnien et al., 2011b; Schaeper et al., 2010).

A
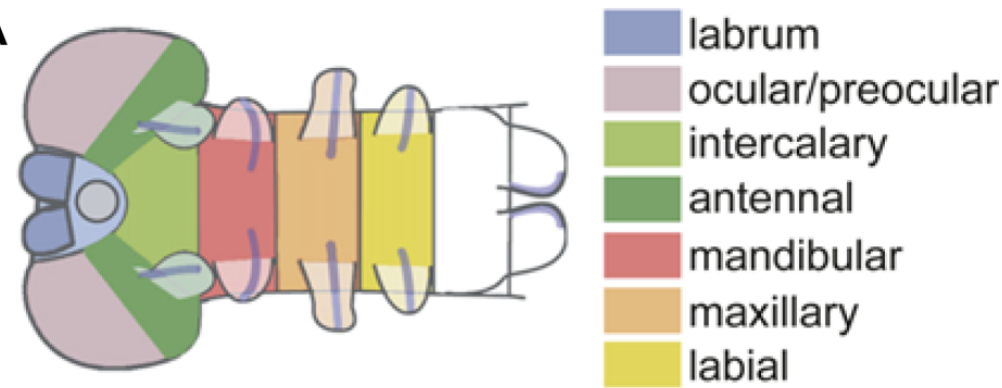

B

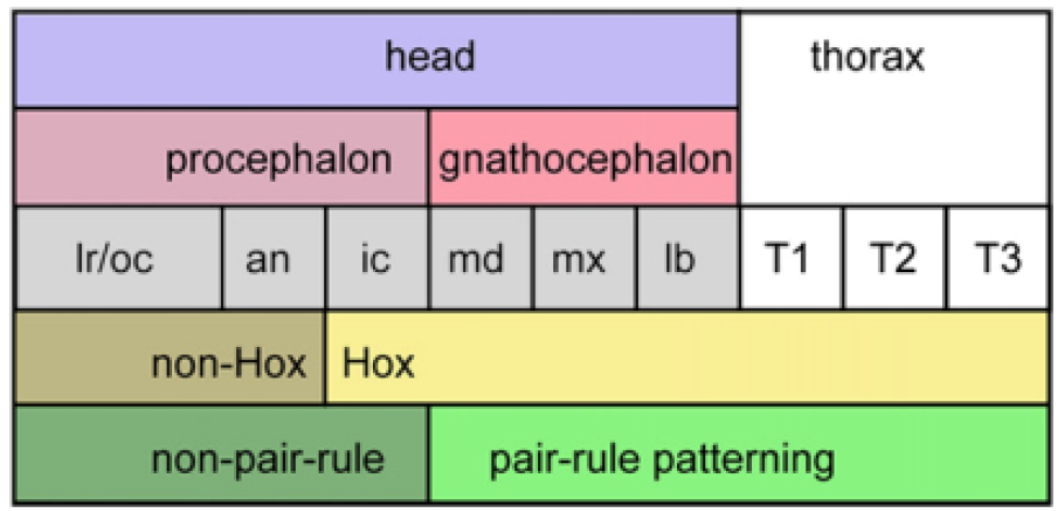

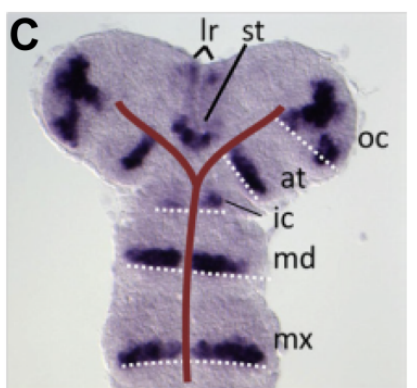

Figure 2.2 Composition and patterning of the Tribolium head. (A) The Tribolium head is composed of the posterior gnathocephalon and the anterior procephalon. The gnathocephalon comprises the gnathal segments, which give rise to the labium, maxillae, and mandibles, which are important for feeding. The procephalon is built by the intercalary segment, antennal segment, ocular/preocular region, and the labral region. This region gives rise to the antennae, compound eyes, ocelli, stomodeum, labrum, and the brain. The procephalon is mainly involved in sensing and subsequent integration of information. (B) The gnathocephalon is patterned like the trunk by Hox genes and pair-rule genes. However, the procephalon 
shows no expression of these genes, except for the intercalary segment. (C) Using molecular markers, the procephalon could be subdivided further into a segmental region, with trunk-like parasegment boundaries (marked with dashed lines) and an anterior pre-segmental region, which show no trunk-like parasegment boundaries. The red line indicates a split of the Tc-wg expression in the anterior part of the head enclosing the non-neurogenic anterior median region (AMR), which comprise the labral and stomodeal region. (Posnien 2011b, modified): Ir: labral region, oc: ocular region, an: antennal segment, ic: intercalary segment, md: mandibular segment, mx: maxillary segment, lb: labial segment, TS1-3: thoracic segments 13

The anterior pre-segmental region comprises the neurogenic ocular/preocular region and nonneurogenic 'anterior median region' (AMR, see Figure 4.6.1), which will give rise to the stomodeum and the labrum (Kittelmann et al., 2013, Figure 2.2A: blue region). This region was shown to rely on a highly conserved set of genes, which is also expressed in the vertebrate neural plate (Posnien et al., 2011b). Previous studies on the gene regulatory network of this region (Figure 2.3) were able to show that $T c$-six3 is an upstream regulator for this region (Kittelmann et al., 2013; Posnien et al., 2011b; Nico Posnien et al., 2009).

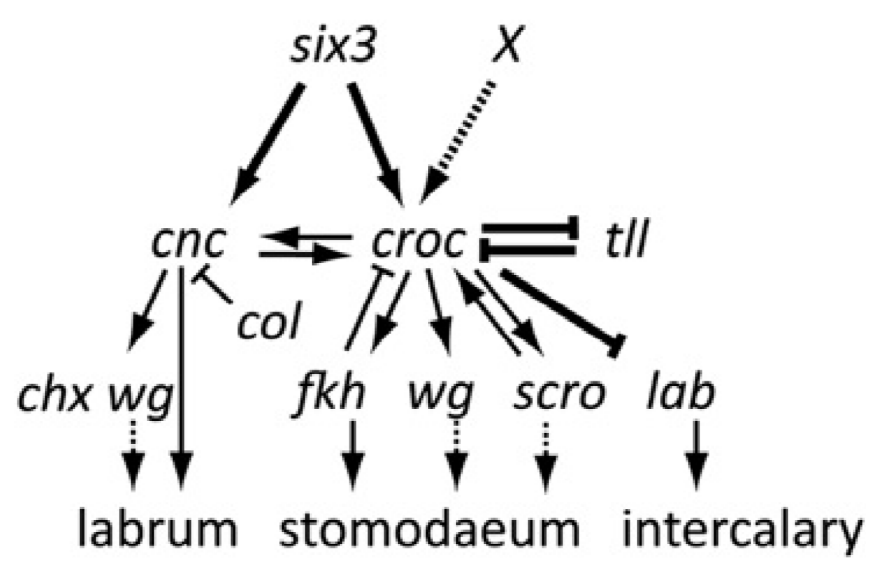

Figure 2.3 Gene regulatory network of the anteriormost head region in Tribolium. Arrowheads indicate activation and crossbars indicate gene repression. Dashed lines indicate hypothetical effects. Tc-six 3 is a cardinal factor for pattering of the anteriormost region. Tc-six 3 is activating the anterior expression of $\mathrm{Tc}-\mathrm{croc}$ within the AMR, while an unknown player ' $X$ ' for the posterior portion is still unknown. (Taken from Kittelmann et al., 2013)

$T c$-six3 activates $T c$-cnc, which is responsible for formation of the anterior portion of the AMR, and Tc-croc, which is responsible for formation of the posterior portion of the AMR. Both transcription factors are required for formation of the labrum. $T c-h k b$ and $T c-t l l$ show no 
terminal gap gene function and are not involved in the formation of the anteriormost region of the head. Further, $T c-c r o c$ regulates $T c-f k h$ and thereby the stomodeum formation instead of $T c-h k b$, as for Drosophila reported. Based on expression patterns it is also hypothesized that $T c$-wingless $(T c-w g)$ is involved in labrum and stomodeum formation. Tc-scarecrow (Tc-scro) is hypothesized to play a role in the development of the stomodeum based on its expression pattern. However, there are still gaps concerning the knowledge of the gene regulatory network of the anterior head region, while the candidate gene approach seems to be exhausted. For example, an unknown player ' $X$ ' that regulates the posterior part of the $T c$-croc domain within the AMR is still missing. Further, it has not been reported, which factor activates $T c$-six 3 (Kittelmann et al., 2013; Posnien et al., 2011b).

\subsubsection{The 'iBeetle' screen - trying to find novel genetic regulators for head}

\section{development}

The knowledge about anterior head pattering gained so far is mainly based on analyzing candidate genes known from Drosophila head development and vertebrate neural plate (Economou and Telford, 2009; Kittelmann et al., 2013; Posnien et al., 2011a, 2011b). However, the candidate gene approach is biased towards conserved gene function (Schmitt-Engel et al., 2015). This approach appears to be exhausted, while it is obvious that important players patterning the anterior head are missing (see above; Kittelmann et al., 2013). To overcome the limitations of the candidate gene approach, a large-scale unbiased RNAi screen was started in 2011 for Tribolium with the aim to identify unknown function of genes, which are involved in essential processes, e.g. head development, muscle formation, and odoriferous gland formation. Several thousands of dsRNA fragments were injected to interfere with gene functions at embryonic as well as at postembryonic stages. The iBeetle screen is bipartite and composed of (1) a larval injection screen addressing genes, which are involved in post-embryonic developmental processes, and (2) a pupal injection screen, which addresses genes involved in embryonic development. The target genes were chosen randomly. In the next years the screen is planned to be finalized, thereby achieving genome-wide coverage (Bucher, pers. communication). The resulting developmental phenotypes are searchable in the online "iBeetleBase" (http://ibeetle-base.uni-goettingen.de; Dönitz et al., 2015, 2013; Schmitt-Engel et al., 2015). In this database an interesting new candidate gene was annotated, which showed a labrum-specific cuticle phenotype, upon pRNAi. The labrum phenotype indicates a function in anterior head development. Thus, this was a promising candidate to further complement the 
anterior head gene regulatory network. Information provided by the iBeetle genome browser (http://bioinf.uni-greifswald.de/gb2/gbrowse/tcas5/) suggested that the targeted gene is an ortholog of the Drosophila fd102C (CG11152), described as foxq2, a member of the forkhead gene family (Lee and Frasch, 2004; Mazet et al., 2003).

\subsection{Forkhead box transcription factor family}

The first forkhead box family member was identified 1989 in Drosophila. Mutant loss-of-function flies showed ectopic spike-shaped head structures, which led to the name of the gene and eventually to the name of the gene family 'fork head' (Benayoun et al., 2011; Hannenhalli and Kaestner, 2009; Lam et al., 2013; Weigel et al., 1989). Forkhead proteins are known to function as transcription factors, which are required for initiation and regulation of transcription (Figure 2.4, Benayoun et al., 2011). All members of the Forkhead box family share the roughly 100 amino acid long Forkhead DNA-binding domain (Benayoun et al., 2011; Carlsson and Mahlapuu, 2002; Hannenhalli and Kaestner, 2009; Lai et al., 1990; Weigel and Jäckle, 1990).

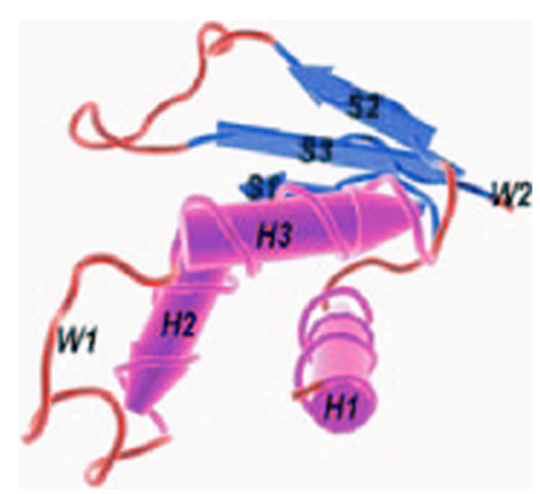

Figure 2.4 Structure of a classical Fox protein domain (FoxQ1). The classical FOX domain is composed of (N-terminal $\rightarrow \mathrm{C}$ terminal): three $\alpha$-Helices $(\mathrm{H} 1 / \mathrm{H} 2 / \mathrm{H} 3)$, three $ß$-strands (S1/S2/S3), and two loops (W1/W2). The conformation resembles butterfly wings and thus coined the nickname 'winged-helix'. (Benayoun et al., 2011, modified)

So far 19 fox subfamilies have been described (Benayoun et al., 2011; Hannenhalli and Kaestner, 2009; Kaestner et al., 2000; Larroux et al., 2008; Mazet et al., 2003; Shimeld et al., 2010), which are represented in more than hundred different species of animal and fungi with over 2000 members. Subfamilies are marked by an alphabetic character as suffix (fox $a-s)$. However, the total number of fox genes and the number of represented families within each species is variable (Benayoun et al., 2011; Shimeld et al., 2010). Fox transcription factors tend to bind to DNA as monomers. Their function in biological processes is diverse ranging from insulin-signaling, 
diabetes, ageing, cancer, vocal learning, chromatin remodeling, to nuclear receptor binding (Benayoun et al., 2011; Hannenhalli and Kaestner, 2009).

\subsection{1 foxq2}

Members of the foxq2 gene family have been first described in 2003 in two different species. The Caenorhabditis elegans gene C25A1.2., although at the time not referred being member of the foxq2 family, was shown to be expressed in nerve cells of the circumpharyngal nerve ring, but showed no phenotype, upon RNAi (Hope, 2003). Further, it has been shown that the foxq2 gene in amphioxus is expressed at the anterior pole at embryonic and larval stages. This was the first study showing the apical expression pattern of the foxq2 genes and, hence, suggesting an important role in AP patterning (Yu et al., 2003). foxq2 subfamily members have been found in a large number of species across the metazoan kingdom (see taxa in Figure 2.5; Chapman et al., 2010; Chevalier et al., 2006; Fritzenwanker et al., 2014; Hope, 2003; Hunnekuhl and Akam, 2014; Koziol et al., 2016; Larroux et al., 2008; Lee and Frasch, 2004; Marlow et al., 2014; Martín-Durán et al., 2015; Martín-Durán and Hejnol, 2015; Mazet et al., 2003; Santagata et al., 2012; Shimeld et al., 2010; Sinigaglia et al., 2013; Tu et al., 2006; Yaklichkin et al., 2007; Yu et al., 2008, 2003; Zhang et al., 2014). Intriguingly, placental mammals lack a foxq2 representative, whereas in other vertebrates, like Danio rerio and the monotreme Ornithorhynchus anatinus, representatives could be found (Shimeld et al., 2010; Yu et al., 2008). 


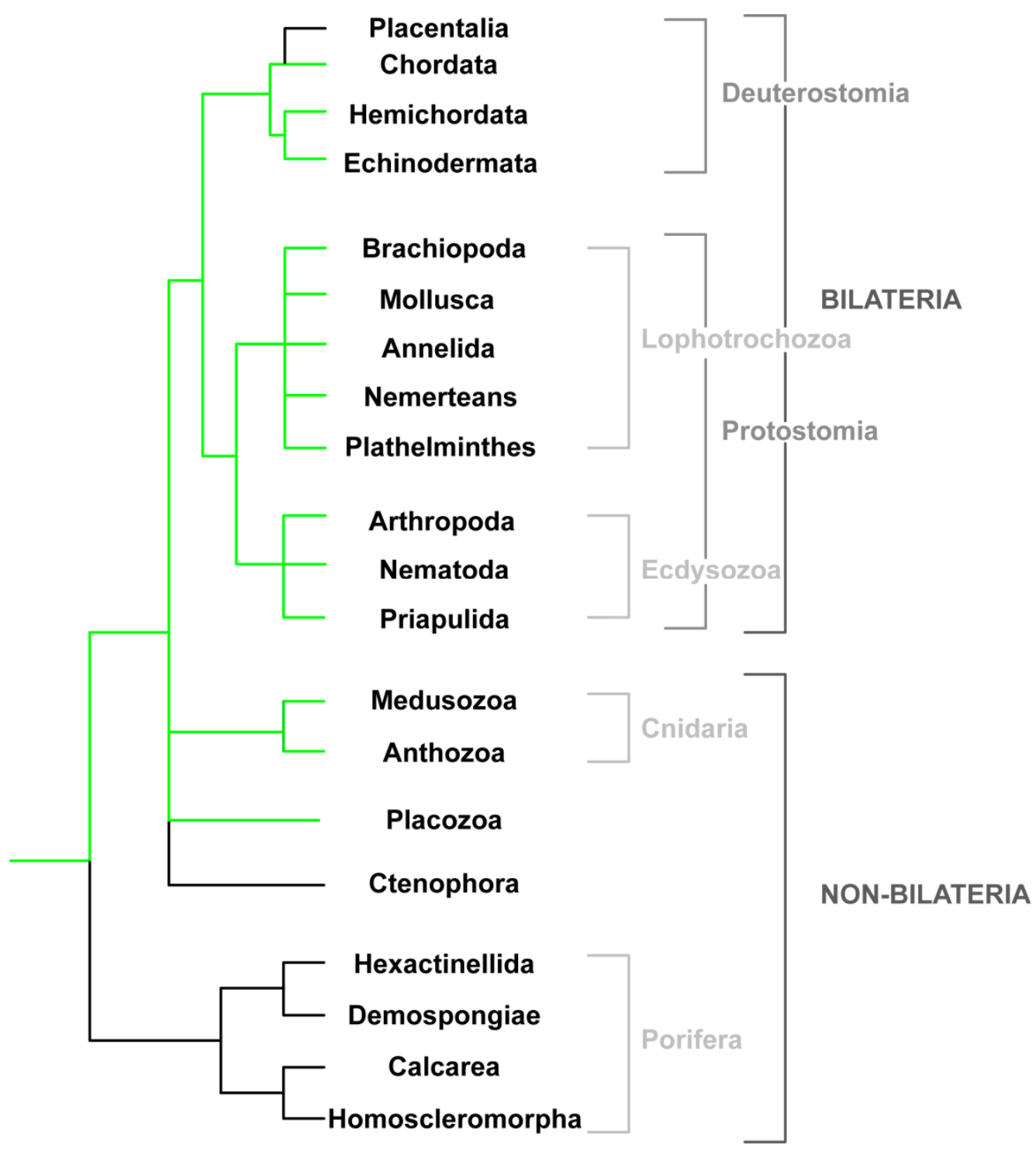

Figure 2.5 foxq2 gene subfamily members are found in almost all phyla of the metazoan kingdom. Phyla showing foxq2 gene subfamily members are marked in green. foxq2 is not represented in the Placentalia and Ctenophora. Pre-metazoan clades like the Porifera lack members of the foxq2 subfamily. (Tree is based on Dohrmann and Worheide, 2013; Prud'homme et al., 2003)

Most of the foxq2 representatives in these different species appear to show comparable expression profiles, patterning a region hypothesized to be homologous (Fritzenwanker et al., 2014; Yaguchi et al., 2008). This homologous region is marked by a set of genes including six3 and $r x$ being conserved across protostome lophotrochozoans, deuterostomes, and cnidarians (Figure 2.6). It has been shown in Nematostella and Platynereis dumerilii (Platynereis) that foxq2 is also part of this conserved pattering system (Marlow et al., 2014; Sinigaglia et al., 2013; Tosches and Arendt, 2013). Further, it has been shown that this conserved set of genes also 
patterns an ancient neurogenic region of different species. In aquatic larval stages of Nematostella, Strongylocentrotus, Terebratalia transversa, and Platynereis these genes are patterning the sensory-neurosecretory apical tuft sometimes also referred to as apical organ (Howard-Ashby et al., 2006; Marlow et al., 2014; Santagata et al., 2012; Sinigaglia et al., 2013; Wei et al., 2009; Yaguchi et al., 2012, 2010, 2008). In the arthropod Strigamia maritima it has been shown that these genes are patterning a neurogenic region similar to the apical organ of other invertebrate marine larvae (Hunnekuhl and Akam, 2014). In Tribolium, the postulated presegmental region contributes to the central complex and the mushroom bodies, which are parts of the protocerebrum (Scholtz and Edgecombe, 2006). It was already shown for Tc-six3 to play a major role in patterning and formation of the mushroom body and the central complex (Posnien et al., 2011b). However, data with respect to foxq2 function is so far only provided for the cnidarian Nematostella vectensis (Nematostella; Sinigaglia et al., 2013) and the echinoderm deuterostome Strongylocentrotus purpuratus (Strongylocentrotus; Range and Wei, 2016; Yaguchi et al., 2012, 2010, 2008). In Nematostella Nvfoxq2a is involved in the development of the aboral region by regulating genes like NvSix3/6 and NvHoxF. In knock-down experiments the overall larval morphology is unaffected but the apical organ size is reduced (Sinigaglia et al., 2013). In Strongylocentrotus, foxq2 is involved in ectodermal patterning by regulating the oralaboral axis specification, via repression by Wnt/ß-catenin signaling and repression of nodal. Knock-down of foxq2 leads only to minor morphological defects, characterized by a slight thickening of the animal plate ectoderm. However, foxq2 knock-down compromises the development of serotonergic neurons and the differentiation of long cilia in the apical organ/apical tuft (Yaguchi et al., 2010, 2008).

However, the expression and function of foxq2 has so far not been characterized in insects. This study promises to provide information about the expression and function of foxq2 in ectodermal and neural development in insects and thereby contribute to reconstruct the conserved anterior patterning gene regulatory network. 


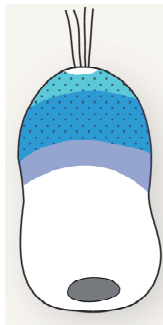

cnidarians

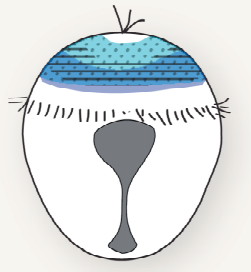

annelids

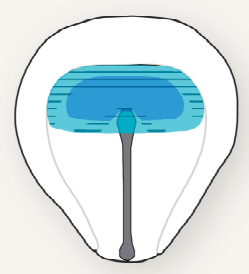

vertebrates

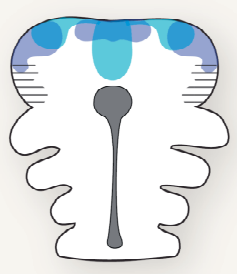

insects $\operatorname{six} 3$

$r x$

$\ominus$ fezf

foxQ2

Figure 2.6 Conservation of an apical patterning gene set in different species across metazoan species. Schematic representation indicating expression of six $3, r x, f e z f$, and foxq 2 at the apical pole of different metazoan species. Nematostella data represents cnidarians, Platynereis data represents annelids, Xenopus laevis data represents vertebrates, and Tribolium data represents the insects. (Taken from Tosches and Arendt, 2013)

\subsection{The arthropod head problem}

\subsubsection{Origin and questions}

The head and the brain were formed in a process called 'cephalization'. Starting point of this process was a common unsegmented bilaterian ancestor. Segments evolved separating the two non-segmental termini, forming the homonomously segmented ancestor of the arthropods. During arthropod cephalization, trunk segments were successively added to the anterior nonsegmental part of the homonomously segmented ancestor to form an anterior tagma. Simultaneously, the ganglia of each segment fused at the anterior pole, building the brain. The different arthropods have varying numbers of segments building the head (Budd and Telford, 2009; Ou et al., 2012; Snodgrass, 1960). This composite structure of the head enabled a high diversification of the arthropod head shape, which allowed the different species to adapt to their environmental conditions and different ecological niches (Posnien et al., 2010). However, the composition of the arthropod head is disputed since decades, a discussion, which is called the 'arthropod head problem' (Budd, 2002; Haas et al., 2001; Jürgens et al., 1986; Posnien et al., 2010; Rempel, 1975; Rogers and Kaufman, 1997; Schmidt-Ott et al., 1994; Schmidt-Ott and Technau, 1992; Scholtz and Edgecombe, 2006). This problem comprises several open questions, but I will focus on the following subset: Is there evidence for the existence of (1) a labral segment, and/or (2) a non-segmental region called 'acron'? (3) Which of them will give rise to the labrum? (4) If there is a non-segmental region, what is its contribution to the adult head? And what is the contribution of the segmental parts to the adult head? 


\subsubsection{Morphogenesis of the insect head}

In order to gain insights into the contribution of certain cells or tissues to a specific region were classically high-invasive experiments, e.g. hot needle cauterization or laser ablation, performed (Jürgens et al., 1986; Posnien et al., 2010; Sander, 1976; Wada, 1965). More recent studies used less invasive methods like analysis of mutants, RNAi phenotypes, marker gene in-situ hybridizations, fuchsin stainings, and membrane stainings to get insights into the Tribolium head fate map (Posnien et al., 2010; Posnien and Bucher, 2010). These analyses challenged the classical hypothesis about the head fate map. This hypothesis proposed that the gnathal segments of the embryonic head perform a dorsal closure similar to the trunk segments. Thus, the antennal, intercalary, and gnathal segments were suggested to contribute to lateral and dorsal parts of the adult head (Figure 2.7). However, the new findings led to the 'bend and zipper' model (Figure 2.8A-D), which predicts complex tissue movements resulting in a different prediction of the head fate map (Figure 2.8E). The new head fate map predicts that the maxillary and labial segment contribute to a minor extent to the dorsal capsule, whereas the other segments give only rise to lateral parts of the adult head capsule. Most of the dorsal head is built by the ocular/preocular region (Posnien et al., 2010).

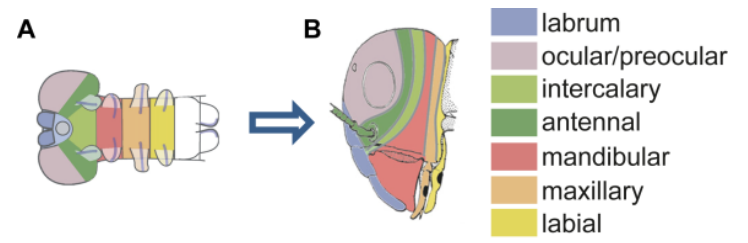

Figure 2.7 Classical head fate map hypothesis. (A) Embryonic segments posterior to the ocular/preocular region undergo trunk-like dorsal closure. (B) These segments each contribute to lateral and dorsal parts of the adult head capsule. The remaining portion of the dorsal head capsule is built by the ocular/preocular region. (Posnien et al., 2010, modified)
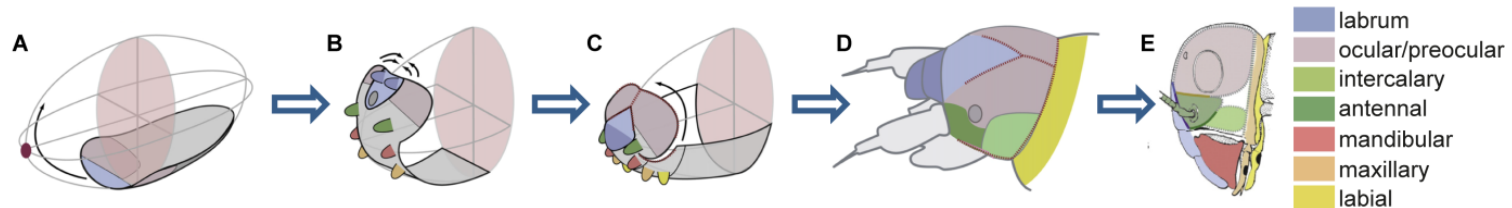

Figure 2.8 The bend and zipper model predicts a new head fate map. (A) The embryonic head anlagen are located at a ventral sub-terminal position within the egg. In the course of development, the germ band elongates towards the anterior pole of the egg. Thus, the head lobes will be bent upwards. (B) The lateral head lobes (ocular/preocular region) approach the embryos dorsal midline and start to fuse, in a zip-like 
manner from anterior to posterior. (C) The labral buds fuse and form the labrum underneath the fused head lobes. The maxillary and labial segment will undergo trunk-like dorsal closure and fuse anteriorly with the head lobes. (D) Preliminary larval head fate map, resulting from the morphogenetic movements predicted by the bend and zipper model and mutant/RNAi phenotype analysis. (E) The data extrapolated to an adult head fate map. In this newly established head fate map, only the post-mandibular gnathal segments contribute to dorsal parts of the cuticle, whereas the more anterior segments only contribute to lateral parts of the head capsule. The dorsal head capsule is primarily built by the ocular/preocular region. The origin of some parts of the head capsule remains unclear (white region). (Posnien et al., 2010; Posnien and Bucher, 2010, modified)

However, also the new head fate map prediction is only preliminary. The bend and zipper model and the resulting new head fate map are based on invasive methods and fixed materials. Moreover, the fate map lacks cellular resolution and is thus limited by its accuracy. Therefore, it was one of my tasks to generate new transgenic lines for in vivo imaging experiments. These new tools should provide the basis to study the morphogenetic movements leading to the adult insect head in non-invasive imaging experiments. Further, cell marking lines should be generated and in the future used to mark the embryonic pre-segmental region and to look for its contribution to the larval head. The ultimate aim is to use these lines to establish an exact and comprehensive head fate map at cellular resolution.

\subsection{Aims}

This study had two aims. On the one hand I wanted to gain more knowledge about the genetic regulation of insect head development, and on the other hand I wanted to shed more light on the embryonic morphogenetic movements, which lead to the larval head.

(I) In order to get new insights into the genetic regulation of the head development, I wanted to decipher the function of Tc-foxq2 in the gene regulatory network of the anterior pre-segmental region using in-situ hybridization in wild-type, pRNAi and gain-of-function embryos. Moreover, I intended to find out the function of $T c$-foxq2 in ectodermal and neural development, via pRNAi and gain-of-function experiments. Finally, I aimed to correlate this Tribolium data with the foxq2 data of other metazoan species in order to perhaps discover a conserved patterning system. 
(II) In order to reveal the morphogenetic movements, which lead to the adult head, it was my aim to provide new transgenic lines expanding the Tribolium in vivo imaging tool kit. Simultaneously, I intended to find a promoter, which is ubiquitously active and drives strong expression at all developmental stages. Further, to provide the tools for the generation of a head fate map at better resolution it was my aim to generate lines, in which a small population of cells could be marked and subsequently tracked. To this end, I wanted to exploit photoactivatable fluorescent proteins for laser-induced cell marking, and the Cre/loxP system for a genetic cell marking system. 
3

\section{Material \& Methods}

\subsection{Animals}

Animals were reared under standard conditions (Brown et al., 2009).

The San Bernadino (SB) wild-type (wt) strain was used for cDNA synthesis, RNAi experiments, whole mount in situ hybridizations and antibody stainings.

The black (Sokoloff, 1974) and the Pig-19 (based on the pearl strain;Lorenzen et al., 2003) strains were used for RNAi experiments only.

The Tc-vermillion ${ }^{\text {white }}\left(v_{w}\right)$ strain (Lorenzen et al., 2002) was used for transgenesis. The line is deficient for Tc-vermillion, which leads to white eyes.

The transgenic line EFA-nGFP drives expression of the green fluorescent protein (GFP) under the

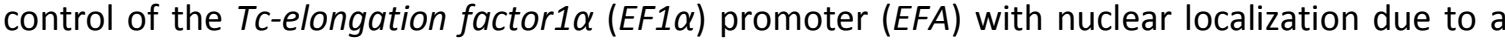
nuclear localization signal (nGFP; El-Sherif et al., 2012; Sarrazin et al., 2012). This line was used for comparison with my newly established nuclear reporter lines (see section 3.10.1).

The transgenic MB-green line (\#176, enhancer trap line G11410 (http://www.geku.base.unigoettingen.de)) was generated in a large-scale insertional mutagenesis screen (Trauner et al., 2009) and marks mushroom body tissue with enhanced GFP (EGFP; Binzer et al., 2014; Koniszewski et al., 2016; Posnien et al., 2011b).

The transgenic brainy line (\#174) marks glial tissue (6xP3-ECFP) and neural cells (EFA-dsRed; Koniszewski et al., 2016; Posnien et al., 2011b). These lines were used for in vivo analysis of larval brain defects.

The Cre (causes recombination) recombinase driver line \#16 (generated by J. Schinko) drives Cre expression under the control of the Tc-heat shock protein68 promoter (Tc-hsp68). This line was used to drive the loxP (locus of crossing over (x), P1) responder line, upon heat shock treatment. 


\subsection{Phylogenetic analysis}

Phylogenetic analysis of the Foxq2 proteins was done by using MEGA v.5 (Tamura et al., 2011). The multiple sequence alignment was conducted with the ClustalW algorithm with the preset parameters. Positions containing gaps were eliminated from the dataset. The phylogenetic tree was constructed using the Neighbor-Joining method with the Dayhoff matrix based substitution model (Schwartz and Dayhoff, 1979). Bootstrap tests (Felsenstein, 1985) were conducted using 1000 replicates to test the robustness of the phylogenetic tree.

\subsection{RNAi}

The templates for the non-overlapping double-stranded RNA (dsRNA) fragments were generated via standard PCR from a plasmid template using following primers (including T7 RNA polymerase $\begin{array}{llll}\text { promoter } & \text { sequence): } & \text { Fragment } & \text { Tc-foxq2 } \\ & \text { RNAi_a } & \text { (489 bp): }\end{array}$ 5'-GAATTGTAATACGACTCACTATAGGCTTACTTCAGGACCCGG-3' and 5'-GAATTGTAATACGACTCACTATAGGTCGCTTGTAACAATGCTTGA-3'; Fragment Tc-foxq2 ${ }^{\text {RNAi_b }}$, (197 bp): 5'-GAATTGTAATACGACTCACTATAGgATGTGCAGTAACGAGACTCC $\quad-3^{\prime}$ and 5'-GAATTGTAATACGACTCACTATAGGCTGGGGAAGAGCGGATAGC -3'.

The dsRNA was synthesized using the Ambion $^{\circledR}$ T7 ${ }^{\circledR}$ MEGAscript ${ }^{\circledR}$ kit (lifeTechnologies,Carlsbad,CA,USA). The transcribed dsRNA was extracted via isopropanol precipitation $\left(T c-f o x q 2^{\text {RNAi_a }^{2}}\right.$ ) or phenol/chloroform extraction $\left(T c-f o x q 2^{\text {RNAi_b }}\right.$ ) and dissolved in

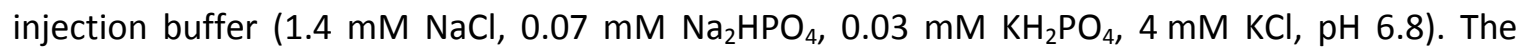
injected dsRNA concentrations for parental RNAi with $T c-f o x q 2^{\text {RNAi_a }}$ and $T c-f o x q 2^{\text {RNAi_b }}$ were $1.0 \mu \mathrm{g} / \mu \mathrm{l}, 1.5 \mu \mathrm{g} / \mu \mathrm{l}$ and $3.1 \mu \mathrm{g} / \mu \mathrm{l}$. If not stated differently, a dsRNA concentration of $1.5 \mu \mathrm{g} / \mu \mathrm{l}$ was used. Pupal injections were performed as previously described (Bucher et al., 2002; N. Posnien et al., 2009). The dsRNA was injected using the FemtoJet ${ }^{\circledR}$ express device (eppendorf, Germany). Cuticles of the L1 larval offspring were prepared as described (Wohlfrom et al., 2006). Head bristle patterns of cuticles were analyzed as described (Schinko et al., 2008).

\subsection{Fixation}

Embryos at an age of $6-26 \mathrm{~h}\left(32^{\circ} \mathrm{C}\right) \mathrm{AEL}$ (for wt single whole mount in situ hybridization (ISH), wt double whole mount in situ hybridization (DISH) and loss-of-function ISH) or 14-18 h AEL (for 
gain-of-function ISH) were fixed using standard protocols (Schinko et al., 2009) with slight modifications: $180 \mu \mathrm{m}$ meshes were used and $2 \mathrm{ml}$ PEMS buffer (0.1 M PIPES, $2 \mathrm{mM} \mathrm{MgCl}, 5 \mathrm{mM}$ EGTA, pH 6.9) in place of fixation buffer.

\subsection{Immunostaining}

\subsubsection{Antibodies}

Immunostaining was performed using the cleaved Drosophila Dcp-1 (Asp216) rabbit antibody (Cell Signaling Technology, Germany) with 1:100 dilution. Anti-rabbit coupled with Alexa Fluor 488 was used for detection with 1:1000 dilution.

\subsubsection{Staining}

The fixed embryos (see section 3.4) were successively rehydrated and freed from methanol, by washing with PBT. The embryos were blocked for one hour at room temperature with $3 \%$ BSA (Fraction V). Afterwards, the primary antibody (in 3\% BSA) was added and incubated $\mathrm{O} / \mathrm{N}$ at $4^{\circ} \mathrm{C}$. After several washing steps with PBT the secondary antibody was added and incubated for 90 min at room temperature. The antibody was removed and the nuclei were afterwards stained with DAPI. The stained embryos were mounted in VECTASHIELD ${ }^{\circledR}$ (Vector Laboratories) to prohibit photobleaching.

\subsubsection{Statistical analysis}

The regions of interest (Figure 4.5: region1, 3 and Figure 4.32: region 2, 3; dashed lines) were all set on the basis of morphological traits. Cell counting was performed using the Fiji cell counter plug-in (Schindelin et al., 2012). The number of apoptotic cells is positively correlated with the age (data not shown). To circumvent potential staging errors, the apoptotic cell number in the posterior procephalon (Figure 4.5 and Figure 4.32: region 3) was counted to normalize the data of interest (Figure 4.5: region 1 and Figure 4.32: region 2). This region was chosen, because it should be unaffected by RNAi experiments based on the expression data and the epidermal L1 larval phenotype. The correction value was calculated by dividing the mean number of apoptotic cells of RNAi embryos (region 3) by the mean number of apoptotic cells in wt embryos (region 3). For the normalization was each single data point for the region 1 (respectively region 2 ) divided by the correction value. 
The normalized data was visualized in a box plot and statistically tested with $R$ v.2.14.2 (http://www.R-project.org/). The dataset was tested for the homogeneity of the variances, via the box plot, and for normal distribution, via the Shapiro-Wilk test. To test for significance, three statistical tests were conducted: Welch t-test, two sample t-test and the Wilcoxon rank-sum test. All three tests show the same levels of significance. Stated $p$-values are based on the Wilcoxon rank-sum test results.

\subsection{Whole mount in situ hybridization}

\subsubsection{Probes}

ISH probes were synthesized with the DIG (Digoxegenin-UTP; ${ }^{D I G}$ ) RNA labeling kit (Roche, Germany) and the Fluorescin (Fluorescin-UTP; ${ }^{\text {FLO }}$ ) labeling mix (Roche, Germany) using the T7 RNA polymerase.

\subsubsection{Staining}

ISH (alkaline phosphatase + NBT/BCIP) and DISH (alkaline phosphatase + NBT/BCIP \& horseradish peroxidase mediated tyramide signal amplification (TSA) reaction: horseradish peroxidase + tyramide-Dylight550 conjugate) were performed as described previously (Oberhofer et al., 2014; Schinko et al., 2009; Siemanowski et al., 2015).

\subsubsection{Co-expression analysis}

The embryos, in the wt DISH assay (Figure 4.8 and Figure 4.9), were staged based on morphological traits and Tc-foxq2 expression pattern. The dashed lines indicating gene coexpression are based on comparisons of the $T c-f o x q 2^{D I G}$ signal (Figure 4.8A) with the signal of $T c-f o x q 2^{\text {FLUO }}$ in the other panels (Figure 4.8B-E and Figure 4.9).

\subsection{Cloning of genes}

Tc-foxq2 full coding sequence (1633 bp; Gen bank accession number: XM_008202469) was obtained from the Tribolium genome browser (http://bioinf.unigreifswald.de/gb2/gbrowse/tcas5/). The following primers were used to amplify the full coding sequence from an embryonic CDNA pool (0-72 h AEL) via standard PCR: 5'- 
ATGTGCAGTAACGAGACTCC-3' and 5'-TTAAGAGTCTGTGGTGTCGG-3'. The Tc-foxq2 full coding sequence was cloned into the PJET1.2 vector (Thermo SCIENTIFIC ${ }^{\mathrm{TM}}$ ).

The Tribolium ortholog of histone $2 A$ variant $(T c-H 2 A v, 387 \mathrm{bp}$, bank accession number: XM_970375) was amplified lacking the stop codon, via standard PCR, with the following primers: 5'-ATGGCTGGTGGCAAAGCAGG-3' and 5'-GACGGGCTGTGAGTGG-3'. The partial coding sequence was cloned into the pJET1.2 vector.

\subsection{Generation of H2Av::EGFP and H2Av::C3PA-GFP}

\section{chimeric reporter proteins}

To generate the chimeric H2Av::EGFP and H2Av::C3PA-GFP nuclear marker proteins, overlap extension PCR was used as described previously (Yolov and Shabarova, 1990; Yon and Fried, 1989).

\subsection{Cloning regulatory regions}

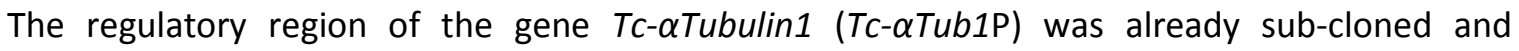
described (Siebert et al., 2008). The regulatory region of Tc-polyubiquitin (Tc-PUbP; 948 bp fragment upstream of the start codon; Primer: 5'-TGTACTTTTCTTTGTCCCAAATGACC-3'and 5'-CTGCAACGACACAAAAAATTACTT-3') and Tc-ribosomal protein subunit3 (Tc-rps3P; 700 bp upstream of the start codon; Primer: 5'-TGTCAAACCACAAACATAAAAAATAG-3' and 5'-TTTGACGTTCTAAATGGAAAAGG-3') were obtained from the Tribolium genome browser (see section 3.7) and isolated from genomic DNA of $S B$ adults. Amplified sequences were sub-cloned in the pSLfa1180fa shuttle vector. The regulatory regions were then cloned into the transformation vector, $5^{\prime}$ upstream of the H2Av::EGFP chimeric marker protein and the SV4O polyadenylation signal. For detailed sequences and vector maps: see section 7.15-.20. 


\subsection{Transgenesis}

\subsubsection{Constructs}

All donor constructs were stably integrated into the genome using the piggyBac vector pBac[3xP3-gTc'v] (Lorenzen et al., 2003, 2002). Plasmids:

[3xP3-gTc'v;Tc'hsp68-Tc'foxq2], [3xP3-gTc'v;Tc' $\alpha$ Tub1P- Tc'H2Av::EGFP], [3xP3-gTc'v;Tc'PUbPTc'H2Av::EGFP], [3xP3-gTc'v;Tc'rpS3P-Tc'H2Av::EGFP], [3xP3-gTc'v;Tc' $\alpha$ Tub1P-C3PA-GFP], [3xP3-

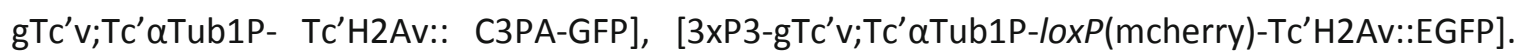
For detailed sequences and vector maps: see section 7.15-.28.

\subsubsection{Germline transformation}

Germline transformation was performed as described previously (Berghammer et al., 1999; Schinko et al., 2012), with slight modifications: the injection buffer was different (see section 3.3) and the TcasHylper (generated by S. Dippel) was used as helper construct. The TcasHylper helper construct carries the mammalian codon-optimized hyperactive transposase open reading frame (Yusa et al., 2011), which is flanked by the regulative sequence of the Tribolium Tc-hsp68 promoter.

\subsubsection{Transformation marker and marker detection}

Tc-vermilion was used and detected as transformation marker as described previously (Lorenzen et al., 2002; Schinko et al., 2012).

\subsection{Heat shock treatment}

All lines for heat shock experiments were kept at $32^{\circ} \mathrm{C}$. Heat shock treatments of embryos (for gain-of-function cuticle preparations: 0-24 h AEL, 9-13 h AEL, 14-20 h AEL, 20-25 h AEL; for gainof-function ISHs: 9-13 h AEL) and pupae were performed as described previously (Schinko et al., 2012) for ten minutes at $48^{\circ} \mathrm{C}$. 


\subsection{Photoactivation}

Photoactivation was performed by using the LSM510 (ZEISS) with the corresponding ZEISS software (v.3.2 SP2). Larvae and pupae were fixed on a microscope slide with Fixogum (Marabu, Germany) and photoactivated using a 20x (air) objective. Embryos were mounted in Voltalef $10 \mathrm{~S}$ Halocarbonoil and photoactivated using a 40x (oil) objective. For photoactivation the $351 \mathrm{~nm}$ and the $364 \mathrm{~nm}$ (both 100\% transmission) UV-Laser line were used simultaneously. Using the software embedded 'Bleach control' tool were the region of interest (ROI), laser lines ( $351 \mathrm{~nm}$, $364 \mathrm{~nm}$ ), scan number (1), and the number of iterations (30x) set. The experiment was at the end conducted with single scans (scan speed 1) with a frame size of $512 \times 512$ pixels, resulting in a total excitation of $207.73 \mu \mathrm{s} /$ pixel per activation cycle. The signal induced by photoactivation was observed using the $488 \mathrm{~nm}$ argon laser with a $505 \mathrm{~nm}$ long pass filter for detection.

\subsection{Image documentation and processing}

Cuticle preps were imaged as described (Wohlfrom et al., 2006). L1 larval brain imaging was performed as described previously (Posnien et al., 2011b). Immuno- and DAPI stainings were imaged using the LSM510 (ZEISS). Stacks of DAPI stained embryos, and stacks of in vivo imaged L1 larval brains were processed in Amira v.5.3.2 (FEI) and displayed as 'voltex' projections. ISHs were imaged using the Axioplan2 (ZEISS, Germany), 10x and 40x objectives, and recorded with the ImagePro v.6.2 software (Media Cybernetics). Individuals of the cell marking lines and the different nuclear reporter lines were imaged with the LSM510 (ZEISS). In vivo imaging videos were recorded using either the ZEISS Lightseet Z.1 (Strobl et al., 2015; Strobl and Stelzer, 2014) or the LSM780 (ZEISS) with the ZEN software (ZEISS). If not stated differently stacks were processed and visualized as average or maximum projections using Fiji (Schindelin et al., 2012). All images were level-adjusted and assembled in Photoshop CS (Adobe). All figures were imported into Illustrator CS5 (Adobe) for labeling and formatting. 


\section{4}

\section{Results}

\subsection{Tc-foxq2 - a novel player in anterior head development of Tribolium}

\subsection{1 iB_03837 targets the Tribolium ortholog of Foxq2}

The iBeetle screen identified an RNAi-induced L1 larval cuticle phenotype which showed labrum specific defects at a high penetrance (Dönitz et al., 2015; Schmitt-Engel et al., 2015). This phenotype was caused by parental RNAi by injecting the iB_03837 dsRNA fragment into female pupae of the pig19 strain, which were crossed with black males. The iB_03837 dsRNA fragment targets a part of the coding sequence of the gene TC004761 (Tcas_OGS 3.0; GenBank accession number: XM_008202469) In order to identify the orthologs of the targeted gene, a phylogenetic tree was built. Phylogenetic analysis revealed that TC004761 is the Tribolium ortholog of Foxq2 (Tc-Foxq2; Figure 4.1). As expected, Tc-foxq2 clustered together with the protostome orthologs. Interestingly, the Tribolium ortholog is more similar to the Platynereis ortholog, compared to the arthropod (Drosophila and Strigamia) orthologs. However, support for the respective branches is comparably low. 


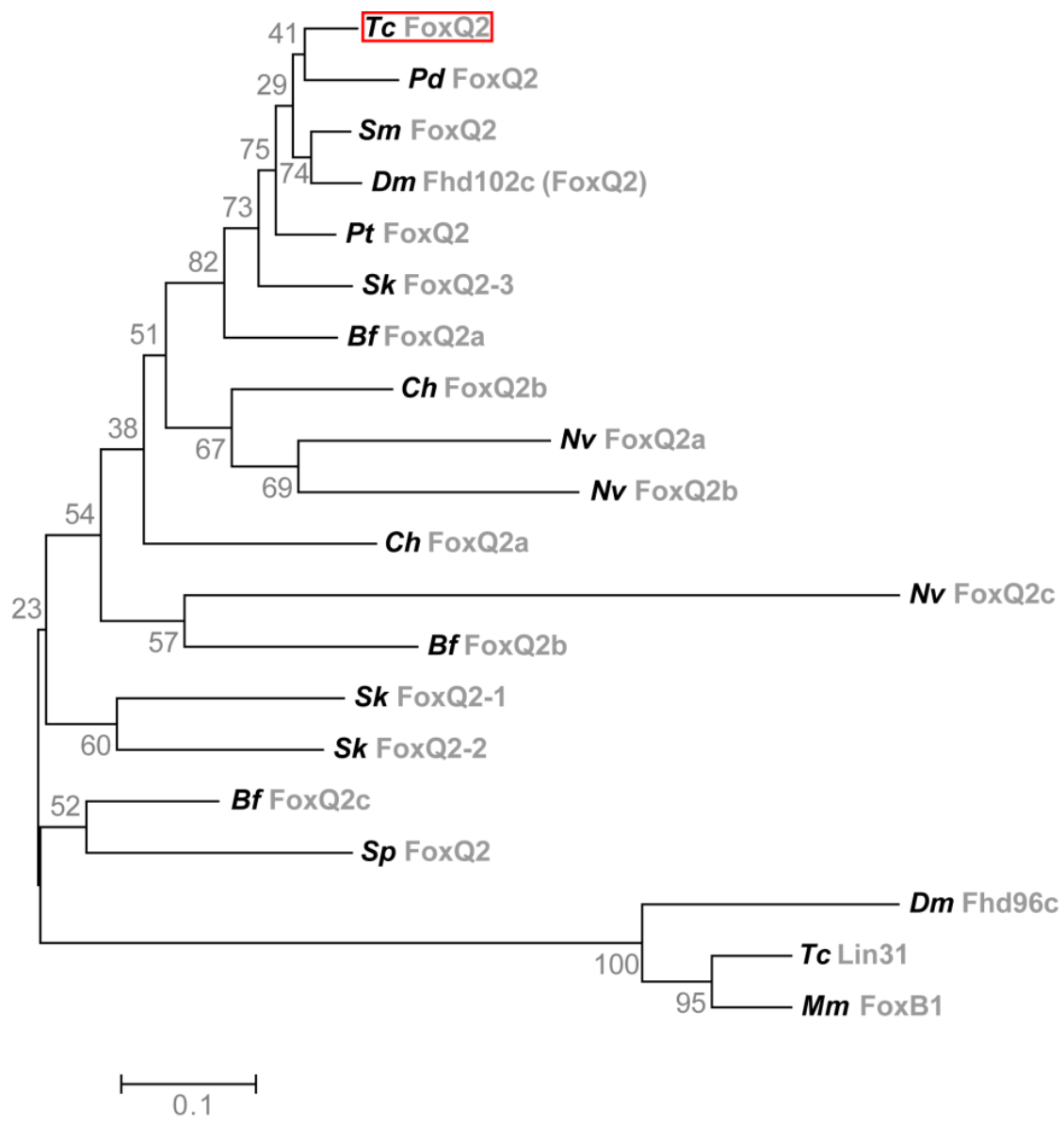

Figure 4.1 Phylogenetic tree of Foxq2 proteins within the Metazoa. Tc-foxq2 encodes for a Foxq2 protein, which clusters together with the protostome orthologs. Interestingly, Tc-Foxq2 is related more closely to the annelid ortholog than to the orthologs of arthropods, which Tribolium is a member of. Shown is a Neighbor-Joining tree with bootstrap values (grey numbers). Tc: Tribolium castaneum, Pd: Platynereis dumerilii, Sm: Strigamia maritima, Dm: Drosophila melanogaster, Pt: Parasteatoda tepidariorum, Sk: Saccoglossus kowalevskii, Nv: Nematostella vectensis, Ch: Clytia hemisphaerica, Bf: Branchiostoma floridae, Sp: Strongylocentrotus purpuratus, Mm: Mus musculus.

\subsubsection{Tc-foxq2 knock-down phenotype in ectodermal tissue}

\subsubsection{Confirmation of the epidermal $T c$-foxq2 phenotype found in the iBeetle screen}

I performed $T c$-foxq2 pRNAi using the $S B$ strain to test the reproducibility of the labrum phenotype that was found under high-throughput conditions in the iBeetle screen. All of the analyzed L1 knock-down cuticles showed the same epidermal phenotype that had been previously described in the iBeetle screen. The cuticles showed either a size-reduced or 
completely absent labrum (Figure $4.2 B^{\prime}, C^{\prime}$ ). Thus, the repetition of this experiment could confirm the Tc-foxq2 pRNAi induced labrum phenotype that was annotated in the iBeetle screen. No other specific cuticle phenotypes were detected.
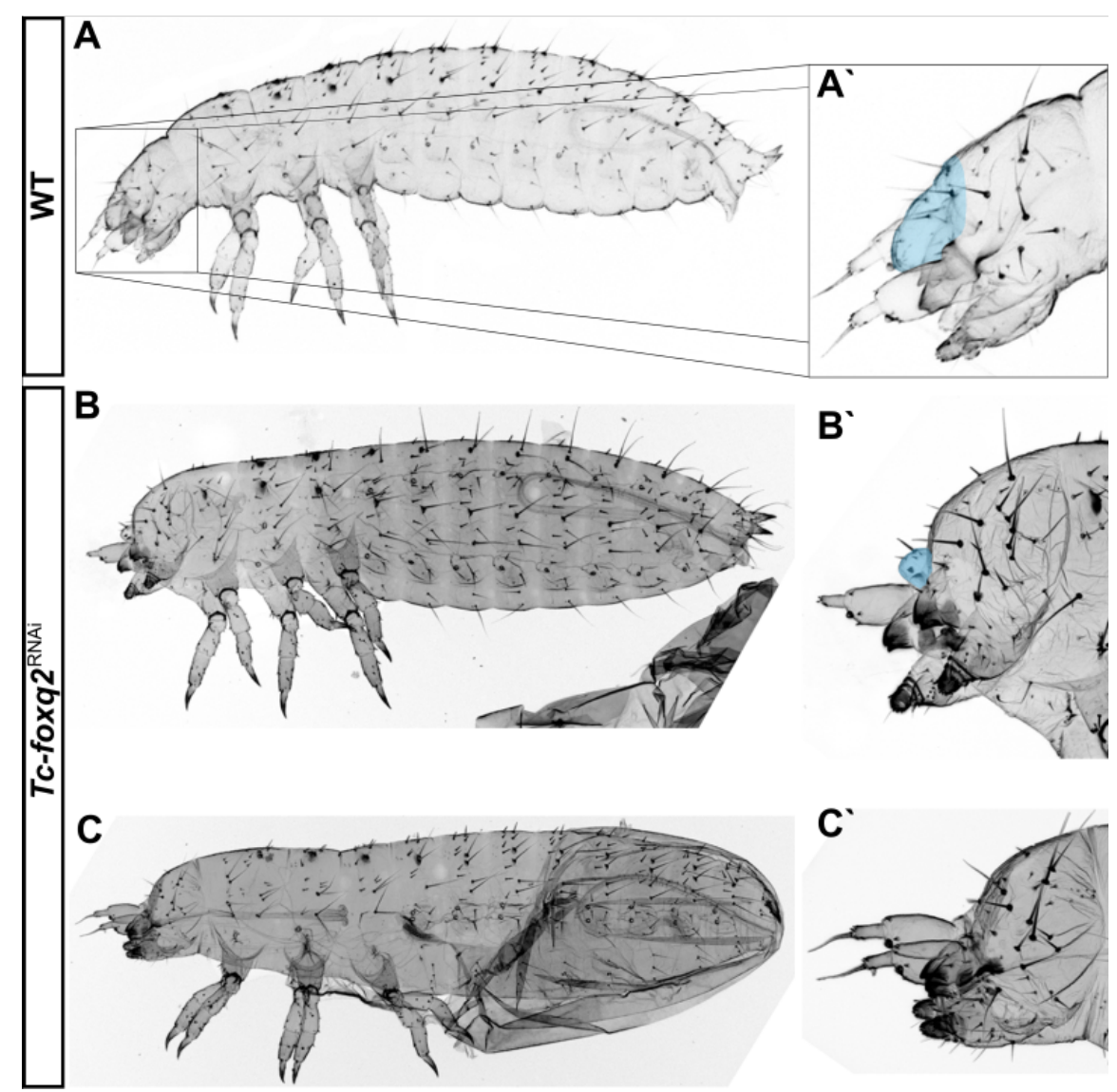

Figure 4.2 Qualitative analysis of Tc-foxq2 pRNAi reveals a labrum-specific phenotype in L1 larvae. All

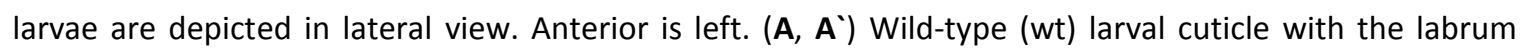
marked in blue (A'). (B-C') $T C$-foxq $2^{\text {pRNAi }}$ cuticles show a labrum, which is either strongly reduced in size (B, B') or completely absent (C, C').

\subsubsection{Tc-foxq2 pRNAi affects the labrum and anteriormost setae}

To get a precise and quantitative data set of the epidermal L1 phenotype, I performed detailed Tc-foxq2 pRNAi experiments (raw counts and exact percentages are displayed in Table S7.1-.4). Further, I generated two new non-overlapping dsRNA fragments (Tc-foxq2 $2^{\text {RNAi_a }}$ and $T c-f o x q 2^{\text {RNAi_b }}$ ) to exclude off-target effects (Qiu, 2005). Both are targeting parts of the $T c$-foxq2 coding sequence. Comprehensive analysis of $L 1$ larval cuticles (Figure 4.3A: Tc-foxq $2^{\text {RNAi_a }}$ : $n=838$; B: $\left.T c-f o x q 2^{\text {RNAi }-b}: n=313\right)$ showed that most of the larvae have defects specific to the head 
(Figure 4.3A, B). The proportion of eggs, which did not contain cuticles and eggs that only contained cuticle remnants were comparable (Figure 4.3A, B) and within in the range that is seen in RNAi experiments. Notably, the proportion of wt cuticles upon pRNAi using Tc-foxq $2^{\text {RNAi_b }}$ was considerably higher (Figure 4.3B) compared to Tc-foxq2 ${ }^{\text {RNAi_aa }}$ (Figure 4.3A). This observation indicates that the $T c-f o x q 2^{\text {RNAi_a }}$ dsRNA fragment led to a more efficient knock-down of the endogenous Tc-foxq2 mRNA. In cuticles showing the head-specific phenotype (Figure 4.3E, bluemarked region), the labrum setae (setae=long trichoid sensilla; Figure 4.3E, yellow dots), the clypeus setae (Figure 4.3E, orange dots), and the anterior setae of the vertex triplet were affected (Figure 4.3E, red dots). I used these setae as marker indicating the grade of the labrum phenotype and to evaluate the overall strength of the defects. I grouped the head defects into three different classes and quantified them (Figure 4.3C, D). Weak phenotypes showed a decreased labrum size, and one or both of the labral setae were missing (Figure 4.3F). Intermediate phenotypes were marked by a reduced labrum size and one or both of the anterior vertex triplet bristles were missing, indicating additionally more posterior defects (Figure 4.3G). Strong phenotypes ranged from cuticles with a strongly reduced labrum size, with deleted anterior vertex setae, and with maximal one labrum seta and one clypeus seta left to cuticles with a completely absent labrum (Figure 4.3H). The quantification indicates that the quality of head phenotypes was the same for both dsRNA fragments. Only the frequency of each of the head phenotypes was slightly different for both fragments, again indicating that the $T c$-foxq $2^{\text {RNAi_a }}$ dsRNA fragment leads to a stronger RNAi effect.

Asking whether the strongest phenotypes may have been missed, I also tested higher dsRNA concentrations ( $2 \mu \mathrm{g} / \mu \mathrm{l}$ and $3.1 \mu \mathrm{g} / \mu \mathrm{l}$; data not shown) as well double RNAi using both dsRNA fragments together ( $T c-f o x q 2^{\text {RNAi_a }}$ and $T c-f o x q 2^{\text {RNAi }}{ }^{\text {b }}$, each $1.5 \mu \mathrm{g} / \mu \mathrm{l}$; data not shown). None of these variations resulted in a stronger cuticle phenotype.

Taken together, these results showed that knock-down of $T c$-foxq2 results in a highly specific epidermal head phenotype, with defects restricted to the anteriormost region of the head. Furthermore, both non-overlapping dsRNA fragments lead to qualitatively equal phenotypes, indicating that the described phenotype is not due to off-target effects. 


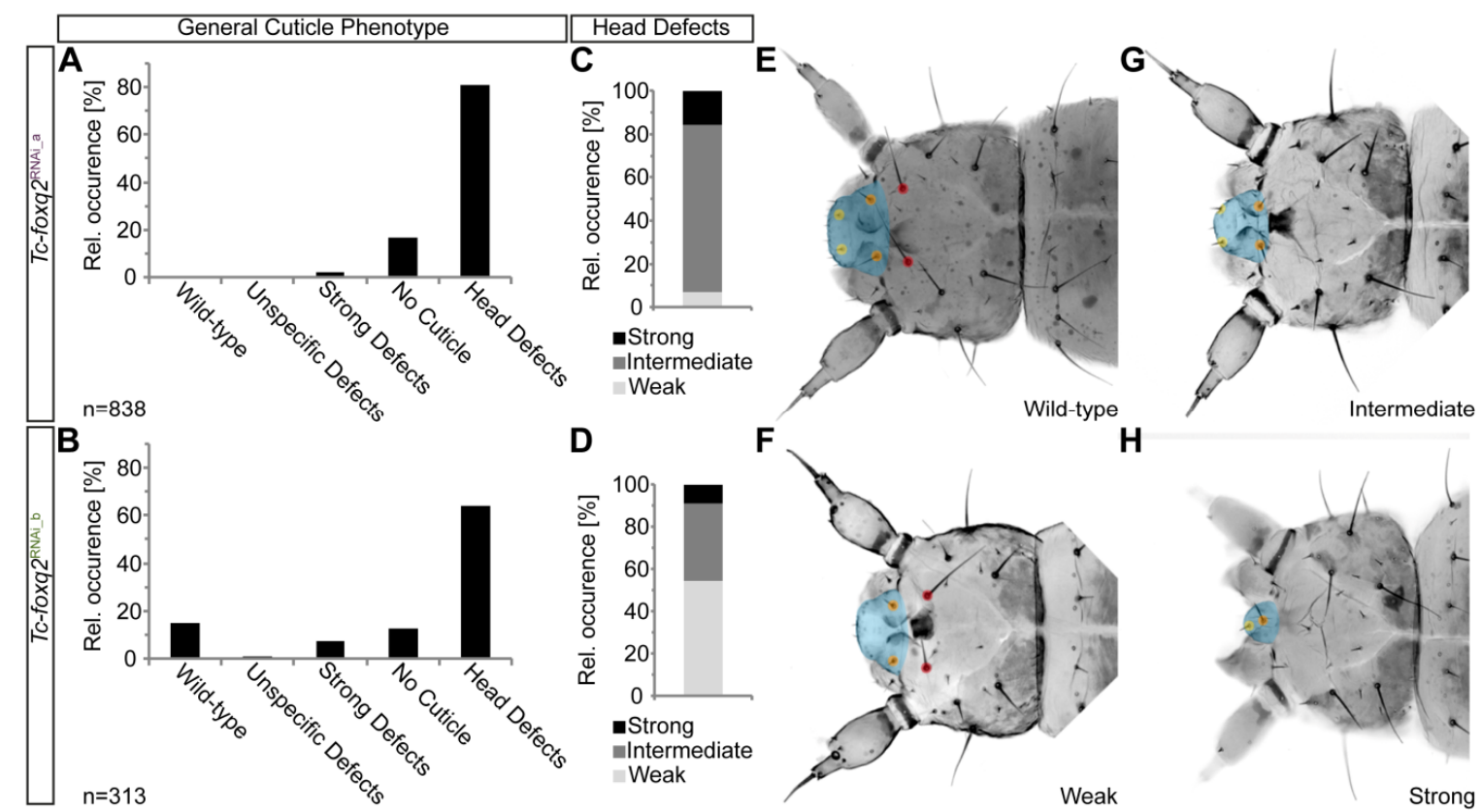

Figure 4.3 Quantitative analysis of the $T c$-foxq $2^{\text {pRNAi }}$ epidermal L1 defects confirms the phenotype and excludes off-target effects. (A, B) Knock-down of $T c$-foxq2 with two non-overlapping dsRNA fragments Tc-foxq $2^{\text {RNAi_a }}(\mathbf{A})$ and $T c-f o x q 2^{\text {RNAi_b }}$ (B) leads to comparable portions of cuticle phenotypes. (C, D) Detailed analysis of head defects shows that the $T c-f o x q 2^{\text {RNAi_a }}$ fragment leads to a qualitatively comparable but quantitatively stronger phenotype, marked by more intermediate $(\mathbf{G})$ and strong $(\mathbf{H})$ head defects. $(\mathbf{E}-\mathbf{H})$ L1 cuticle heads, depicted in a dorsal view, are representing the different classes of head defects. Anterior is left. (E) Wt cuticle with the labrum marked in blue, two labrum setae (yellow dots), two clypeus setae (orange dots) and two anterior vertex setae (red dots). (F) Weak head defect with a reduced labrum and at least one deleted labrum seta. (G) Intermediate head defect is additionally lacking at least one of the anterior vertex seta. (H) Strong head defect with a strongly reduced labrum, one labrum seta and one clypeus seta. The strongest phenotypes show a completely absent labrum and deleted anterior vertex setae.

\subsubsection{The Tc-foxq2 pRNAi L1 epidermal phenotype is not strain specific}

It has been previously described that RNAi-induced phenotypes could depend on the used strain, hence the given genetic background (Kitzmann et al., 2013). To test whether the observed phenotype is only specific to the used strain, I performed Tc-foxq2 pRNAi in two different genetic backgrounds (raw counts and exact percentages are displayed in Table S7.1-.6). To this end, I injected the Tc-foxq $2^{\text {RNAi_b }}$ dsRNA fragment into female pupae of the pig19 strain (see section 3.1) and crossed them with black strain (see section 3.1) males (n=796; Figure 4.4), like 
in the iBeetle screen. The repetition, within the changed genetic background, revealed a comparable general cuticle phenotype, showing high proportions of cuticles with head defects (Figure 4.4B). Furthermore, the quality of observed head defects was the same as in previous experiments. The $T c$-foxq $2^{\text {RNAi_b }}$ dsRNA fragment led to higher frequencies of intermediate and strong head defects in the pig19/black genetic background compared to the results in the $S B$ genetic background (Figure 4.4A, compare D to E). However, compared to the pRNAi experiment using the $T c$-foxq $2^{\text {RNAi_a }}$ dsRNA fragment in $S B$, the expressivity of the head defect was very similar (Figure 4.4 compare E to F). The observed defects in different genetic backgrounds are qualitatively equal and appeared in comparable proportions indicating that the epidermal phenotype after Tc-foxq2 pRNAi is not strain specific. 

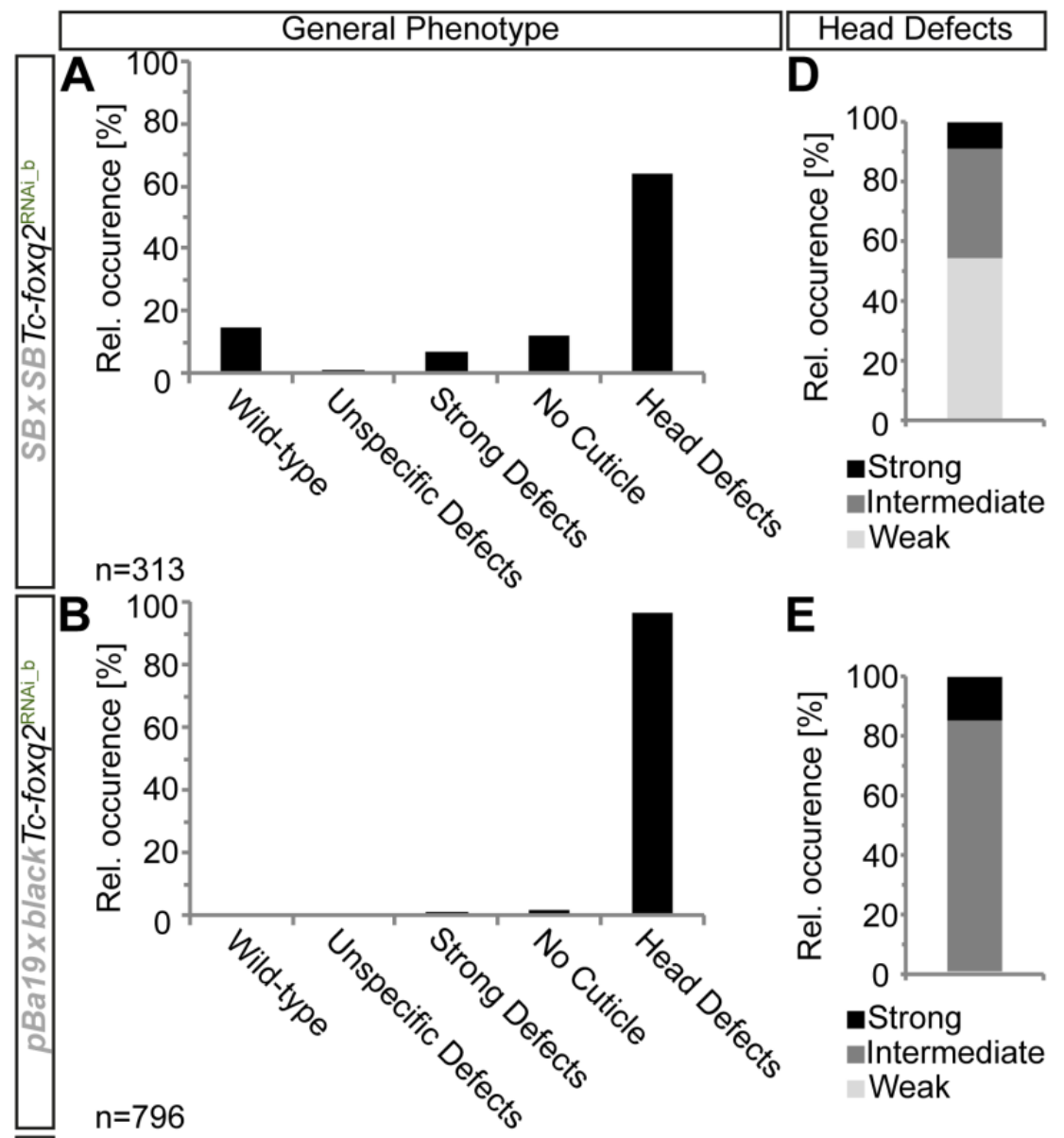

E
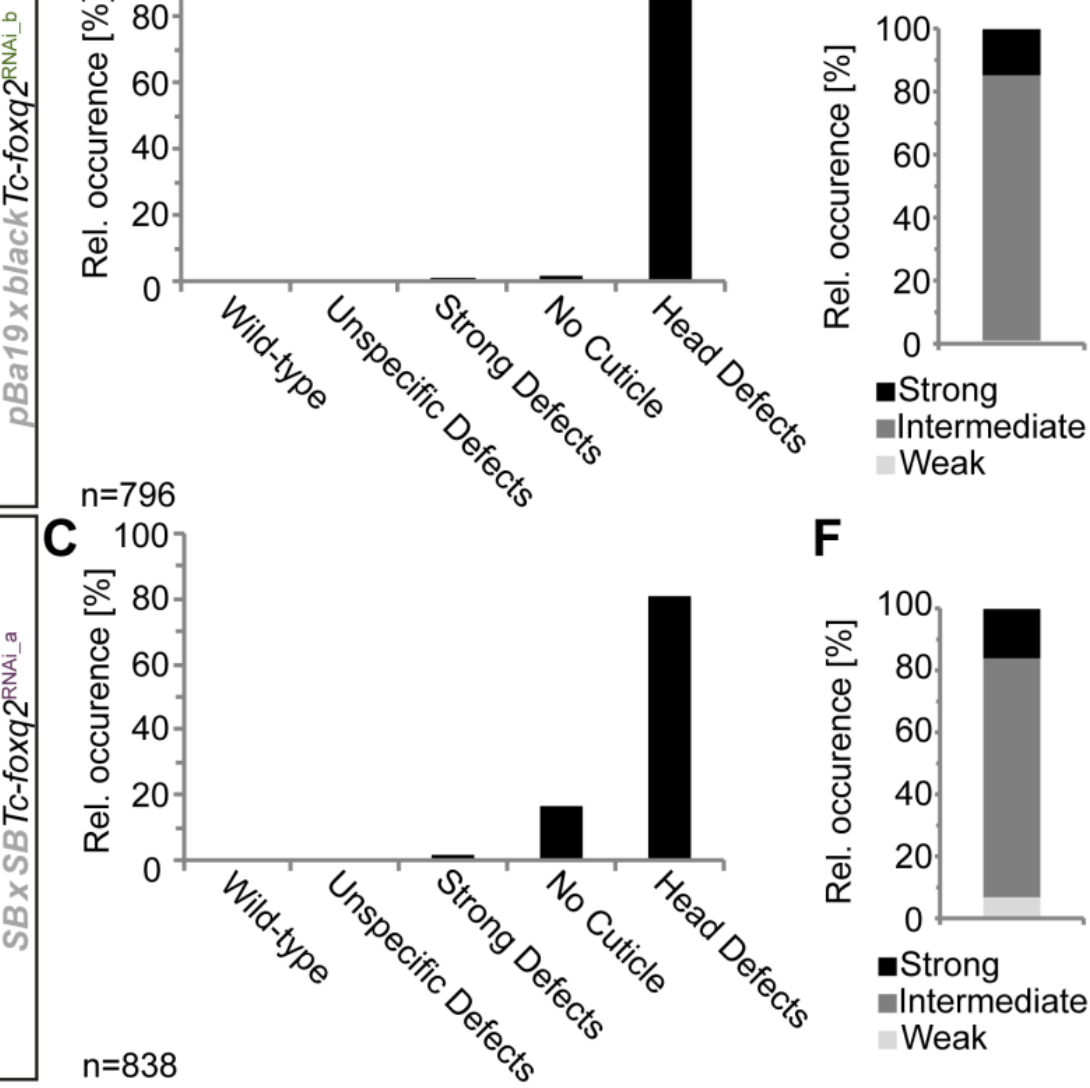

F

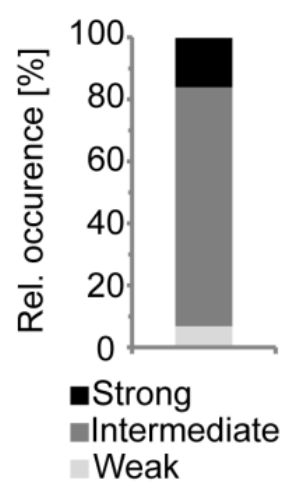

Figure 4.4 Quantitative analysis of the $T c-f o x q 2^{\text {pRNAi }}$ epidermal L1 phenotypes in two different strains shows no considerable strain specific effects. (A, B) Knock-down of $T c-f o x q 2$ using the $T c-f o x q 2^{\text {RNAi_b }}$ dsRNA fragment in the SB strain leads to a distribution of L1 larval general cuticle phenotypes (A), which is comparable to the distribution of phenotypes in L1 offspring, when the same dsRNA fragment is injected in pupal pig19 females, which were crossed to black males (B). (B, C) The distribution of the general phenotype classes gets even more similar when compared to the RNAi experiment using the Tc-foxq $2^{\text {RNAi_a }}$ dsRNA fragment in the $S B$ strain (compare $\mathbf{C}$ and $\mathbf{B}$ ). (D-F) Also the frequency and quality of head defects is comparable throughout the RNAi experiments in different genetic backgrounds. (Data from A, D, C, F taken from Figure 4.3) 


\subsubsection{Tc-foxq2 pRNAi epidermal phenotype results presumably from an increased} apoptotic cell death rate

In order to find out how Tc-foxq2 pRNAi acts to interfere with labrum development, I asked when the labrum is decreased in size. To this end, I visualized the embryonic morphology by staining the nuclei with DAPI and compared labral bud sizes of $T c-f o x q 2^{\text {pRNAi }}$ embryos versus wt embryos (Figure $4.5 \mathrm{~A}$, blue marker). The comparison revealed that $T c$-foxq $2^{\text {pRNAi }}$ embryos showed a decreased labral bud size from fully elongated germ band stages onwards (Figure $\left.4.5 A_{b^{\prime}, d^{\prime}}\right)$. 
A

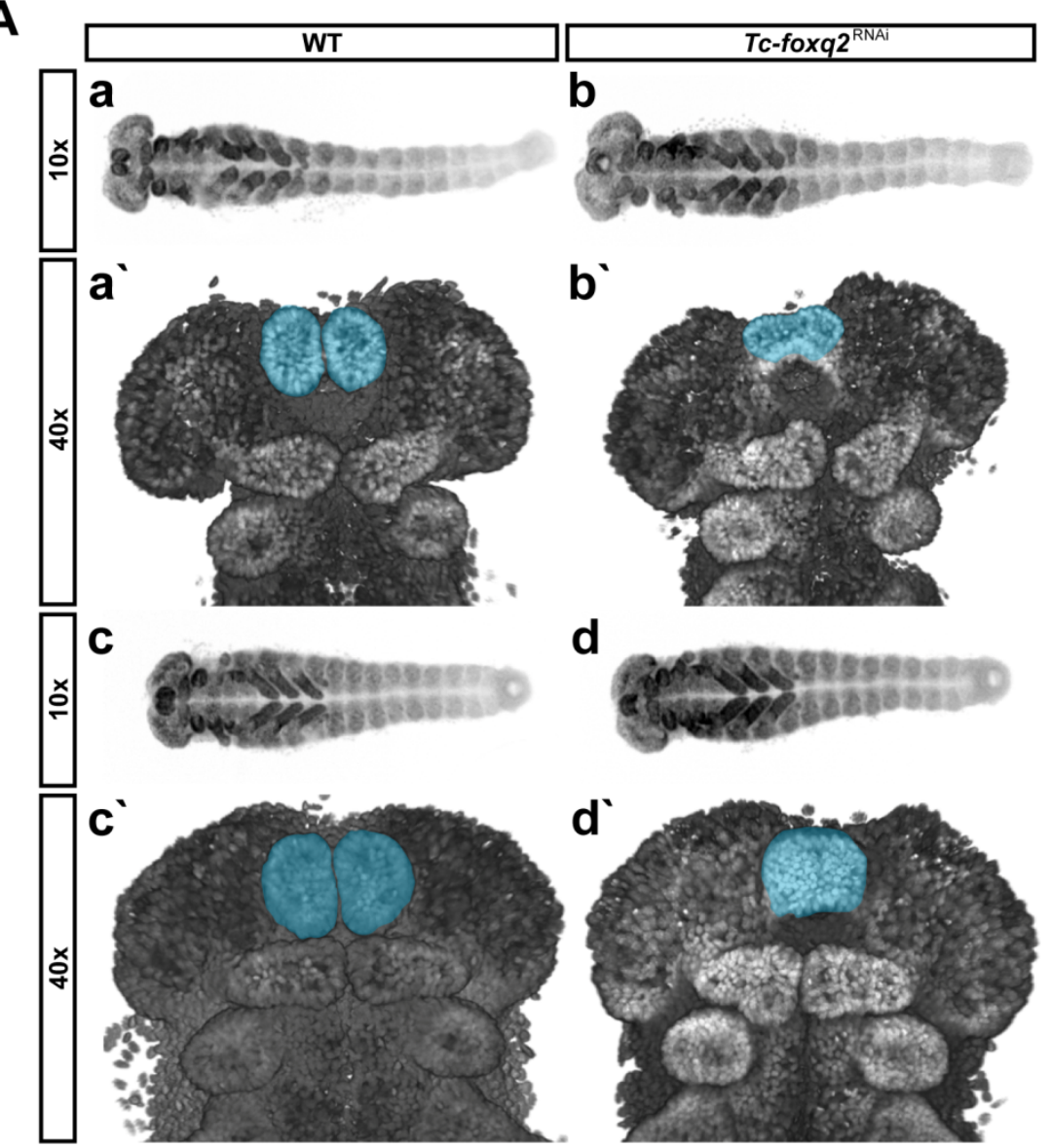

B
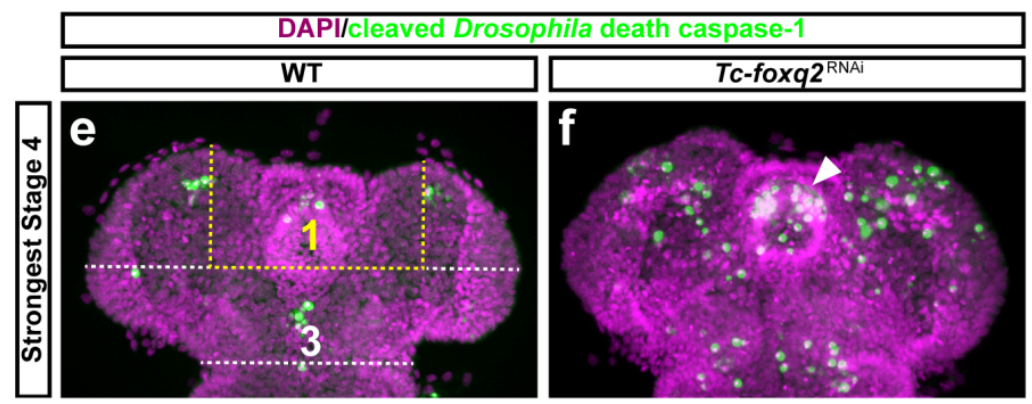

C

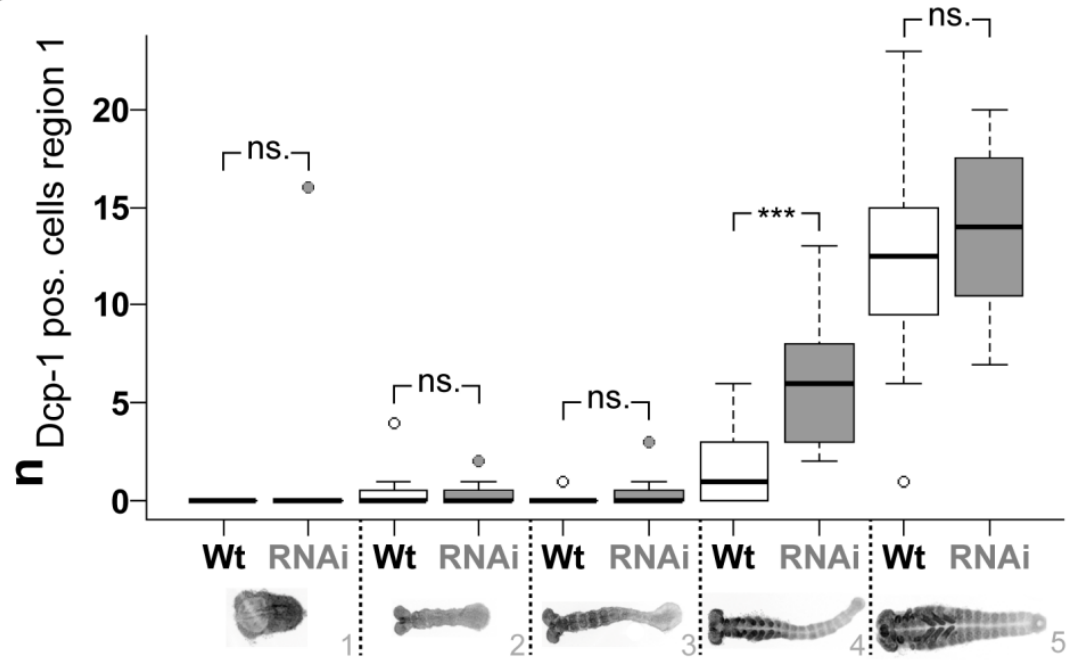

Stage 
Figure 4.5 Qualitative analysis of the embryonic $T c$-foxq $2^{\text {pRNAi }}$ phenotype and its correlation with cell death rates. (A) Morphology of wt $\left(\mathbf{A}_{\mathbf{a}}, \mathbf{A}_{\mathbf{a}}, \mathbf{A}_{\mathbf{c}}, \mathbf{A}_{\mathbf{c}}\right)$ and $T c-f o x q 2^{\text {pRNAi }}\left(\mathbf{A}_{\mathbf{b}}, \mathbf{A}_{\mathbf{b}}, \mathbf{A}_{d}, \mathbf{A}_{\mathbf{d}}\right)$ embryos is visualized by nuclear staining (DAPI, grey). Anterior is left in 10x panels $\left(\mathbf{A}_{\mathrm{a}-\mathrm{d}}\right)$ and up in $40 \mathrm{x}$ panels $\left(\mathbf{A}_{\mathrm{a}}\right)$. The labrum is

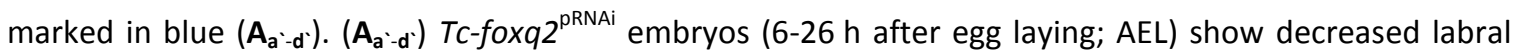
buds, which appear to fuse prematurely $\left(\mathbf{A}_{\mathbf{b}}, \mathbf{A}_{\mathbf{d}^{\prime}}\right)$. (B) For quantification of cell death rates, a region of interest and a control region were defined. The region of interest is the labral region (region $1, \mathrm{ROI} 1$, yellow dashed lines). The posterior procephalic region was used for data normalization (region 3, white dashed lines). Morphology of wt $\left(\mathbf{B}_{\mathrm{e}}\right)$ and $T c-f o x q 2^{\text {pRNAi }}\left(\mathbf{B}_{\mathrm{f}}\right)$ embryos is visualized by nuclear staining (DAPI, magenta). Apoptotic cells are monitored by antibody staining (Cleaved Drosophila death caspase-1 (Dcp-1) - Alexa Fluor 488; green). ( $\mathbf{B}_{\mathrm{e}}, \mathbf{B}_{\mathrm{f}}$ ) The fully elongated $T c$-foxq $2^{\text {pRNAi }}$ germ band with the most apoptotic cells within the labral region (ROI 1, marked with dashed lines) show apparently more marked cells $\left(\mathbf{B}_{\mathrm{f}}\right)$ than the strongest representative of the wt embryos within this stage and region $\left(\mathbf{B}_{\mathrm{e}}\right)$. $\left(\mathbf{B}_{\mathrm{f}}\right)$ The $T c-f o x q 2^{\text {pRNAi }}$ embryo show an accumulation of apoptotic cells within the labral buds (arrowhead). (C) Box plot depicting the normalized number of apoptotic cells (y-axis) versus five different embryonic stages, subdivided in untreated wt and $T c$-foxq $2^{\text {pRNAi }}$ embryos. The ROI 1 values are normalized with the region 3 values (Be). Brackets display grade of significance. Germ rudiments (stage 1) to intermediate elongating germ bands (stage 3), as well as early retracting germ bands (stage 5) showed no significant increase in the number of apoptotic cells within the ROI 1 of $T c$-foxq $2^{\text {pRNAi }}$ embryos (stage $1: p=0.33$ (wt: $\mathrm{n}=3, \mathrm{RNAi}: \mathrm{n}=7$ ), stage 2: $p=0.63$ (wt: $\mathrm{n}=11$, RNAi: $\mathrm{n}=12$ ), stage 3: $p=0.19$ (wt: $\mathrm{n}=9$, RNAi: $\mathrm{n}=19$ ), stage 5: $p=0.15$ (wt: $\mathrm{n}=12$, RNAi: $\mathrm{n}=11)$ ). However, fully elongated germ bands (stage 4) showed significantly more apoptotic cells $(p=0.00041)$ in $T c$-foxq $2^{\text {pRNAi }}$ embryos $(n=15)$ compared to untreated ones $(n=17)$. ns.: not significant

RNAi-induced labrum phenotypes could be due to altered embryonic cell proliferation or cell death rates (Kittelmann et al., 2013; Siemanowski et al., 2015). It has been shown that decreased cell proliferation rates, controlled by Notch signaling were the reason for a decreased labral bud size in one case (Siemanowski et al., 2015). To this end, I performed Tc-serrate (Tc-ser; marker for Notch signaling) whole mount in situ hybridization (ISH) in Tc-foxq2 $2^{\text {pRNAi }}$ embryos (6$26 \mathrm{~h}$ after egg laying; AEL) and looked for an alteration of the expression profile. However, ISH experiments in $T c$-foxq $2^{\text {pRNAi }}$ embryos showed no considerable difference in $T c$-ser expression compared to wt embryos (data not shown).

Thus, I asked whether altered embryonic cell death rates could be involved in the observed labrum phenotype. I performed cleaved Drosophila death caspase-1 (Dcp-1) antibody staining in embryos (6-26 h AEL) to visualize and quantify apoptotic cells (Florentin and Arama, 2012; Figure $4.5 B, C$ ). On the basis of morphological traits I defined two regions: Region 1 (Figure $4.5 \mathrm{~B}_{\mathrm{e}}$ : yellow dashed lines, 1 ) was the actual region of interest (ROI), encompassing the labral 
region, while region 3 (Figure $4.5 \mathrm{~B}_{\mathrm{e}}$ : white dashed lines, 3) was the control region, which should show no RNAi-induced changes in cell death rates. The region 3 data set was used to normalize the actual data set in order to exclude unspecific changes of cell death rates (see section 3.5.3 for details). Furthermore, I set up five different groups, representing different embryonic stages (stage 1: germ rudiments, stage 2: early elongating germ bands, stage 3: intermediate elongating germ bands, stage 4: fully elongated germ bands, and stage 5: retracting germ bands (Figure 4.5C, $\mathrm{x}$-axis)). The analysis revealed that fully elongated germ bands showed a significantly increased number of apoptotic cells, upon $T c$-foxq2 pRNAi ( $p=0.00041, \mathrm{n}=15$; Figure 4.5C: stage 4, $\mathrm{B}_{\mathrm{f}}$ : arrowhead; for raw counts see Table S7.7-.8).

These data indicate that not an altered cell proliferation rate but an increased apoptotic cell death rate at early embryonic stages could be a reason for the reduced embryonic labral bud size and the L1 larval cuticle phenotype.

\subsubsection{Tc-foxq2 expression}

\subsubsection{Tc-foxq2 is expressed in a highly dynamic pattern in the anterior head}

It has been previously shown that foxq2 shows a highly conserved expression pattern at the anterior pole of embryonic stages (Chevalier et al., 2006; Fritzenwanker et al., 2014; Hunnekuhl and Akam, 2014; Lee and Frasch, 2004; Martín-Durán and Hejnol, 2015; Santagata et al., 2012; Sinigaglia et al., 2013; Tu et al., 2006; Yu et al., 2003). To test whether this holds true for Tribolium, I conducted different ISH experiments to identify the onset and course of the Tc-foxq2 expression pattern. To facilitate orientation during subsequent data description, a schematic representation of on embryonic head is shown in Figure 4.6.1.

The expression of $T c-f o x q 2$ starts at early embryonic stages at the anterior terminus (Figure 4.6A-C). At later stages it is expressed within the non-neural anterior median region (AMR: for orientation see Figure 4.6.1; Figure 4.6D, E). Subsequent stages show $T c$-foxq2 expression in the labral buds, enclosing parts of the stomodeum, and in the putative neuroectodermal region (Figure 4.6F-L).

These data show that $T c$-foxq 2 is expressed within the AMR, in a highly dynamic expression pattern, and the adjacent neuroectodermal region. Its expression at the anterior pole of the 
embryo confirms the conservation of the anterior function of foxq2. Further, the expression is in line with a direct function in the labrum and indicates a function in neural development.

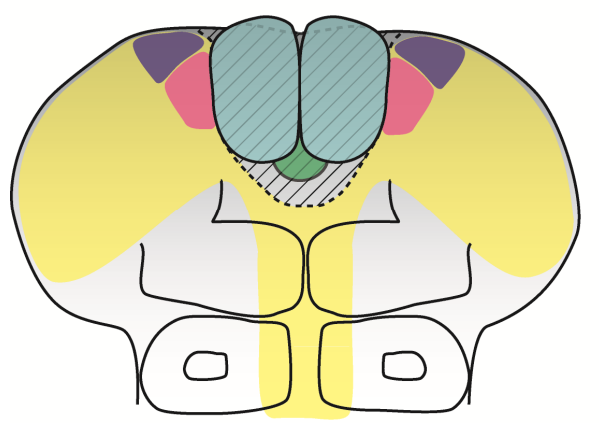

Labrum

\section{Stomodeum}

YJAMR

Pars lateralis

Pars intercerebralis

Neuroectoderm
Figure 4.6.1 Overview of the location of the most relevant embryonic head structures for this study. Anterior is up. The non-neural anterior median region (AMR, striped region) harbors the labral buds (blue)

and the stomodeum (mouth precursor, green). This region is enframed by the neuroectoderm (yellow), in which the central neuroendocrine centers, the pars lateralis (purple) and the pars intercerebralis (pink), are located (de Velasco et al., 2007). (Based on Kittelmann et al., 2013; Posnien et al., 2011b, 2010) 

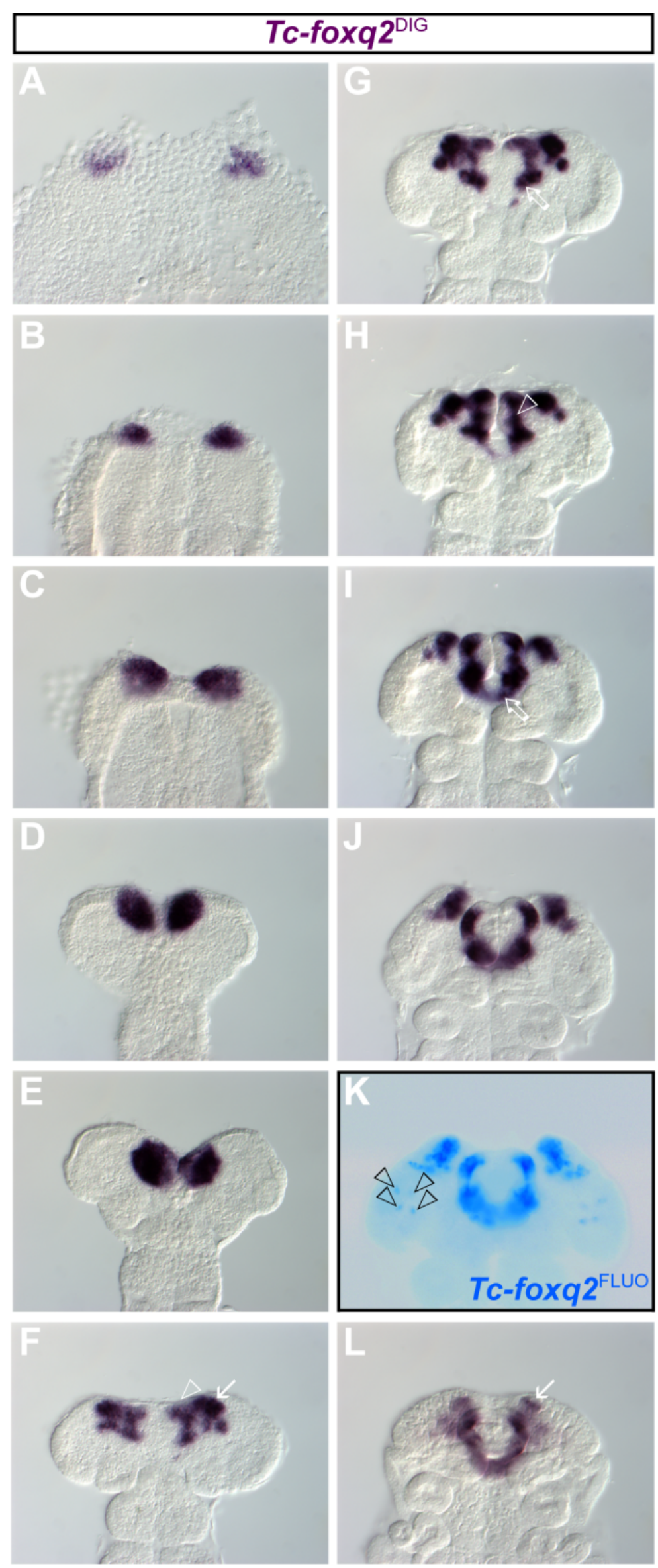
Figure 4.6 Tc-foxq2 is expressed in a highly dynamic pattern at the anterior pole. Anterior is up. Expression of $T c$-foxq2 in wt embryos is monitored by whole mount in situ hybridization (ISH). (A) Tc-foxq2 expression starts with formation of the germ rudiment. (A-E) Early Tc-foxq2 expression is marked by two domains located at the anterior pole, which successively approach each other, probably due to morphogenetic movements, at the embryonic midline. (F) The expression pattern splits into several domains in late elongating germ bands, with expression domains in the putative neuroectoderm (white arrow, presumably including parts of the pars intercerebralis; Posnien et al., 2011b) and in the labral/stomodeal region (white empty arrowhead). (G) The expression domains flanking the prospective stomodeum become more prominent (empty arrow). (H) The anterior median expression domain frames the lateral parts of the labral buds (white empty arrowhead). (I, J) At fully elongated to early retracting germ band stages the two expression domains flanking the stomodeum are linked posteriorly to each other (empty arrow) and the expression domains within the labral buds are getting narrower. (K) Staining with the more sensitive TSA-Dylight550 (Tc-foxq $\left.2^{\mathrm{FLUO}}\right)$ reveals four dot-like expression domains in the ocular region (black empty arrowheads). (L) At retracting germ band stages $T c$-foxq 2 is expressed in a narrow $U$-shape pattern and the neuroectodermal expression domains are reduced in size (white arrow).

\subsubsection{Tc-foxq2 expression might involve a positive autoregulatory feedback loop}

In order to get an impression of the knock-down efficiency upon Tc-foxq2 pRNAi, I performed ISH experiments in Tc-foxq2 ${ }^{\text {pRNAi }}$ embryos. ISH indicated that the signal intensity of $T c$-foxq2 expression seemed to be strongly reduced (Figure 4.7). However, increasing the exposure time revealed that there was an unequal efficiency of the endogenous Tc-foxq2 mRNA knock-down. In early elongating embryos Tc-foxq2 mRNA was almost completely abolished (Figure 4.7B). At later stages the anterior part of the $T c-f o x q 2$ expression domain within the neuroectoderm was highly reduced, whereas the posterior part of the neurogenic $T c$-foxq2 expression domain was still detectable (Figure 4.7F: white arrow). Tc-foxq2 mRNA was still detectable around the stomodeum (Figure 4.7F: empty arrow) and in parts of the labrum (Figure 4.7H: empty arrowhead). Wt expression appeared to be rather equal in strength (Figure 4.7A, C, E, G). Therefore, the difference in knock-down efficiency might result from autoregulation in the respective domains. 


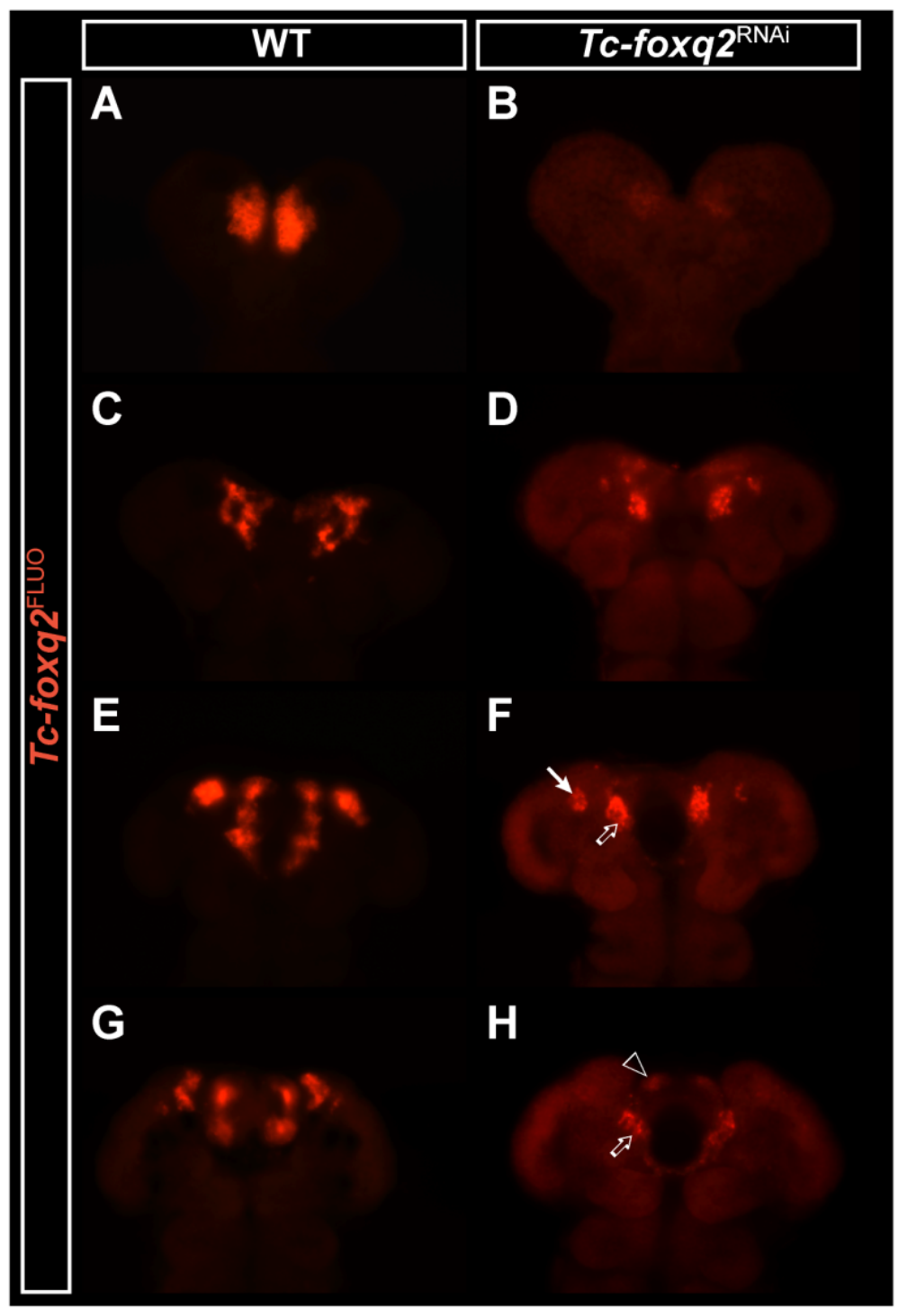

Figure 4.7 Endogenous Tc-foxq2 mRNA is not completely abolished after Tc-foxq2 pRNAi. Anterior is up. Expression of $T c$-foxq2 in wt $(\mathbf{A}, \mathbf{C}, \mathbf{E}, \mathbf{G})$ and $T c-f o x q 2^{\mathrm{pRNAi}}(\mathbf{B}, \mathbf{D}, \mathbf{F}, \mathbf{H})$ embryos is monitored by ISH. Shown are $T c-f o x q 2^{\text {pRNAi }}$ embryos with typical staining. Note that the exposure time had to be strongly increased in order to detect residual staining (see elevated background in B, D, F, H). (B) Upon RNAi treatment $T c$-foxq2 expression is almost completely deleted at early elongating germ band stages. (D) At $T c-f o x q 2^{\text {pRNAi }}$ late elongating germ band stages the posterior portion of the Tc-foxq2 expression is still detectable. (F) Fully elongated germ band stages still show expression in the neurogenic region (arrow) and the stomodeal flanking region (empty arrow). (H) In early retracting $T c$-foxq $2^{\text {pRNAi }}$ embryos is $T c$-foxq2 expression in the anterior part of the labral buds (empty arrowhead) and the stomodeal region (empty arrow) detectable. 


\subsubsection{Uncovering the role of $T c$-foxq 2 within the gene regulatory network of the anterior pre-segmental region}

\subsubsection{Co-expression of Tc-foxq2 with other head patterning genes}

To test for potential $T c$-foxq2 interaction partners within the gene regulatory network of the anterior pre-segmental head development, I looked for mutual co-expression. Genes coexpressed with $T c$-foxq2 are potentially regulating or are regulated by $T c-f o x q 2$. To visualize coexpression, I performed double in situ hybridization (DISH) in wt embryos (6-26 h AEL). For better comparability I grouped these embryos according to their embryonic stage. Expression overlaps are marked with dashed lines.

To test whether $T c$-foxq 2 is potentially interacting with the Wnt/ß-catenin signaling pathway, I analyzed Tc-wingless/wnt1 (Tc-wg, Nagy and Carroll, 1994; Figure 4.8A $\mathrm{A}_{0-6}$ ) and Tc-arrow (Tc-arr, (Bolognesi et al., 2009); data not shown) for co-expression with Tc-foxq2.

$T c-w g$ is a segment polarity gene and ligand of the canonical Wnt signaling pathway. The coexpression analysis of $T c$-foxq 2 and $T c-w g$ showed that there is only an expression overlap at retracting germ band stages in the anterior portion of the labral buds (Figure $4.8 A_{5,6}$ ). $T c$-arr is a co-receptor of $T c-w g$ and therefore also member of the segment polarity genes. In contrast to $T c-w g, T c$-arr is ubiquitously expressed throughout the embryonic development and therefore is covering the $T c$-foxq2 expression pattern entirely (data not shown).

Previous studies showed that sine oculis homeobox homolog 3 (six 3 ) is co-expressed with foxq2 in various species (Fritzenwanker et al., 2014; Hunnekuhl and Akam, 2014; Marlow et al., 2014; Martín-Durán et al., 2015; Santagata et al., 2012; Sinigaglia et al., 2013; Tu et al., 2006; Wei et al., 2009). Thus, I tested whether Tc-optix/six3 (Tc-six3), a transcription factor and major regulator of AMR and central complex development in Tribolium (Nico Posnien et al., 2009; Posnien et al., 2011b), shares also expression domains with $T c$-foxq2 (Figure 4.8B $\mathrm{B}_{0-6}$ ).

$T c$-foxq 2 and $T c$-six 3 expression are largely overlapping from early stages on (Figure 4.8B $\mathrm{B}_{0}$ ). In intermediate elongating germ bands, Tc-foxq2 shows no co-expression with Tc-six3 (Figure $4.8 \mathrm{~B}_{2}$ ). During the course of development they are co-expressed in parts of the AMR, the labral 
buds, and in the putative neurogenic region (Figure $4.8 \mathrm{~B}_{0-6}$ ). The previously described conserved co-expression of foxq2 and six 3 is also represented at most of the analyzed Tribolium developmental stages. However, this holds not true for intermediate elongating germ bands, showing mutually exclusive expression of both factors.

I also tested Tc-cap'n'collar (Tc-cnc), a transcription factor involved in labrum development and proper mandible formation (Coulcher and Telford, 2012; Economou and Telford, 2009; Kittelmann et al., 2013), for co-expression with $T c$-foxq2 (Figure 4.8C $\mathrm{C}_{0-6}$ ). $T c$-cnc and $T c$-foxq2 show an almost complete overlap in their expression profile at elongating germ band stages (Figure $4.8 \mathrm{C}_{0-2}$ ). At later embryonic stages the expression overlap of these genes is restricted to the non-neural part the labral/stomodeal region (Figure $4.8 \mathrm{C}_{3-6}$ ).

TC-scarecrow (Tc-scro ( $n k 2.1$ ortholog)), a transcription factor with roles in proper labrum formation and foregut development (Kittelmann et al., 2013; Posnien et al., 2011b), was also tested for overlapping expression with $T c$-foxq2 (Figure 4.8D $\mathrm{D}_{0-6}$ ). $T c-f o x q 2$ expression covers the complete $T c$-scro expression domains at early elongating germ band stages (Figure $4.8 \mathrm{D}_{0}$ ). In further elongated embryos $T c$-foxq2 expression is partially overlapping with the anterior parts of the Tc-scro domains (Figure $4.8 \mathrm{D}_{1-2}$ ). At later stages Tc-scro expression shows overlaps with $T c$-foxq2 expression only in the lateral part of the AMR (Figure $4.8 D_{3}$ ) or additionally in small areas of the anterior neurogenic region (Figure $4.8 \mathrm{D}_{4-6}$ ).

I tested Tc-crocodile ( $T c$-croc) for co-expression with $T c$-foxq2, due to its role as a transcription factor in AMR formation, and formation of the stomodeum (Economou and Telford, 2009; Kittelmann et al., 2013; Figure 4.8 $\left.\mathrm{E}_{0-5}\right)$. At early germ band stages Tc-croc mRNA is co-localized with $T c$-foxq2 mRNA in latero-anterior parts of the AMR (Figure 4.8 $\mathrm{E}_{0-3}$ ). At later stages they are co-expressed in the posterior portion of the labral buds and around lateral and posterior parts of the stomodeum (Figure $4.8 \mathrm{E}_{4-5}$ ). 


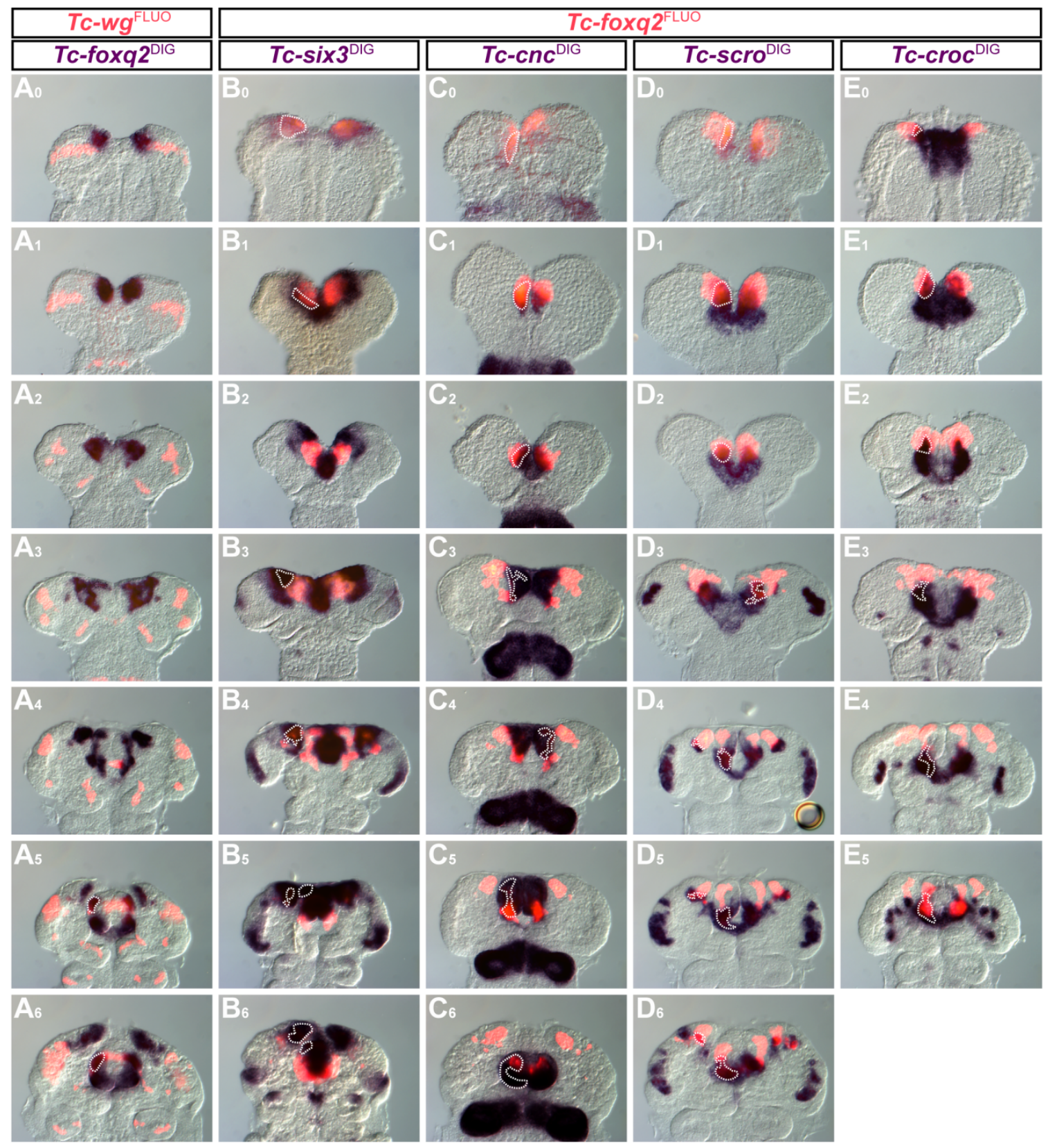

Figure 4.8 Co-expression analyses of $T c$-foxq2 and anterior head patterning genes I. Anterior is up.

Expression is visualized by double-ISH (DISH), using NBT/BCIP (blue) and TSA-Dylight550 (red). For better comparison and potential TSA signal quenching effects by NBT/BCIP staining, Tc-foxq2 was stained using NBT/BCIP in $\mathbf{A}_{\mathbf{0}-6}$. Co-expression is indicated with dashed lines. $\left(\mathbf{A}_{\mathbf{0}-6}\right)$ Until fully elongated germ band stages no $T c$-foxq2/Tc-wg co-expression is detectable $\left(\mathbf{A}_{0-4}\right)$. At later stages $T c$-foxq2 is co-expressed with $T c-w g$ in the anterior portion of the labral buds $\left(\mathbf{A}_{5-6}\right) .\left(\mathbf{B}_{0-6}\right) T c-f o x q 2$ and $T c-s i x 3$ are completely overlapping in their expression at germ rudiment stages $\left(\mathbf{B}_{0}\right)$. In early elongating germ bands the coexpression is limited to a narrow lateral stripe of the AMR $\left(\mathbf{B}_{1}\right)$. Intermediate germ bands show a mutually

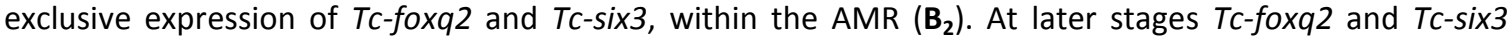
expression are overlapping within the neurogenic region $\left(\mathbf{B}_{\mathbf{3}-4}\right)$. In early retracting germ bands and at later stages $T c$-foxq2 and Tc-six3 expression additionally overlap in the anterior portion of the labral buds $\left(\mathbf{B}_{5-6}\right)$. 
$\left(\mathbf{C}_{0-6}\right) T c-c n c$ expression is almost completely covering the expression of $T c-f o x q 2$ at early embryonic stages $\left(\mathbf{C}_{\mathbf{0 - 2}}\right)$. At later stages the co-expression is restricted to parts of the labral/stomodeal region $\left(\mathbf{C}_{3-6}\right)$. $\left(\mathbf{D}_{0-6}\right)$ Tc-scro is partially co-expressed with Tc-foxq2 within the anterior part of the AMR at early embryonic stages $\left(\mathbf{D}_{\mathbf{0 - 2}}\right)$. In late elongating germ bands the co-expression is restricted to a narrow lateral stripe of the AMR $\left(\mathbf{D}_{3}\right)$. Later stages show co-expression of Tc-scro and Tc-foxq2 in the posterior portion of the labral buds, the stomodeum flanking region, and small areas of the neurogenic region $\left(\mathbf{D}_{4-6}\right)$. Tc-croc expression is partially overlapping with $T c$-foxq2 within antero-lateral parts of the AMR at early stages $\left(\mathbf{E}_{0-2}\right)$. At later stages of development the $T c-f o x q 2 / T c$-croc co-expression is restricted to the stomodeum flanking region and the posterior portion of the labral buds $\left(\mathbf{E}_{3-5}\right)$.

I tested Tc-retinal homeobox (Tc-rx), a transcription factor involved in neuroectodermal development and labrum differentiation (Posnien et al., 2011b), for co-expression with Tc-foxq2 (Figure 4.9A $\mathrm{A}_{-6}$ ). Co-expression of $T c-f o x q 2$ and $T c-r x$ starts at late elongating germ band stages within parts of the neurogenic region (Figure $4.9 A_{3}$ ). Later stages additionally show expression overlaps in the anterior part of the labral buds (Figure 4.9A $A_{5-6}$ ).

Tc-chx is known to be a transcription factor marking parts of the AMR, marking the neuroendocrine pars intercerebralis and being involved in anterior brain development (Koniszewski, 2011; Posnien et al., 2011b), therefore I tested co-expression with Tc-foxq2 (Figure $\left.4.9 \mathrm{~B}_{0-5}\right)$. Tc-chx/Tc-foxq2 co-expression starts at early stages within the AMR, and is later on located in the labral region/labral buds, and within the neuroectodermal region (Figure $\left.4.9 \mathrm{~B}_{0-5}\right)$. The partial expression overlap of $T c-c h x$ and $T c-f o x q 2$ in the neuroectoderm is confirming that $T c-f o x q 2$ is also at least partially marking the pars intercerebralis.

Potential co-expression of Tc-forkhead (Tc-fkh (foxa ortholog)) with Tc-foxq2 was analyzed (Figure $\left.4.9 \mathrm{C}_{0-4}\right)$, because of its role as marker gene for the stomodeal part of the AMR, and its connection to the stomodeum formation (Kittelmann et al., 2013; Schoppmeier and Schröder, 2005). Only retracting germ bands show a small overlap of expression, flanking the stomodeum (Figure $4.9 C_{4}$ ).

Tc-six4 co-expression was analyzed (Figure $4.9 \mathrm{D}_{0-4}$ ), because it is marking the insect head placode, and it is involved in proper formation of the anterior larval head (Posnien et al., 2011a). At the onset of Tc-six4 expression, there is no overlap with the Tc-foxq2 expression domains (Figure 4.9Do). However, at later stages, Tc-six4 activity is partially overlapping with Tc-foxq2 activity at the anterior part of the neuroectoderm (Figure $4.9 D_{1-4}$ ). 
In order to ask whether Tc-foxq2 is interacting with the Notch signaling pathway, I analyzed coexpression of $T c$-foxq2 with $T c$-ser (Figure 4.9 $\mathrm{E}_{0-4}$ ), and Tc-mindbomb 1 (Tc-mib1; data not shown). Tc-ser is a ligand of Tc-Notch within the Notch signaling pathway and required for proper labrum formation (Siemanowski et al., 2015). The analysis revealed that there is a partial overlap of $T c$-ser and $T c$-foxq2 within the AMR at early germ band stages (Figure $4.9 \mathrm{E}_{0-1}$ ). At later stages $T c$-ser is co-expressed with $T c$-foxq2 in the lateral part of the labral buds (Figure $4.9 \mathrm{E}_{2-4}$ ).

Tc-mib1 is an E3 ubiquitin ligase of the Notch signaling pathway and required for proper labrum formation as well (Siemanowski et al., 2015). Tc-mib1 is expressed ubiquitously throughout embryonic development (Siemanowski et al., 2015) and therefore at all embryonic stages of interest overlapping and potentially interacting with $T c$-foxq2 (data not shown).

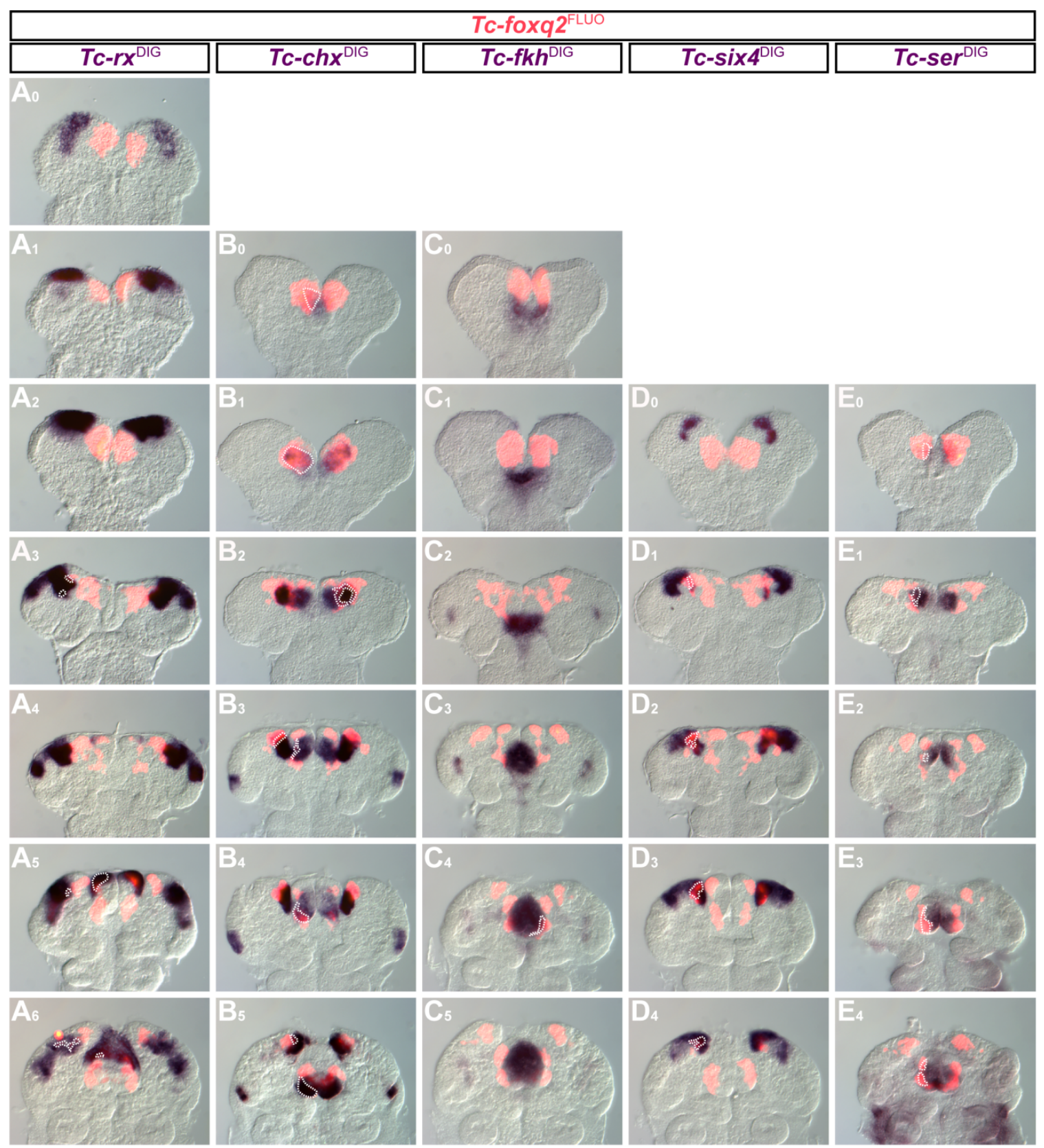


Figure 4.9 Co-expression analyses of $T c$-foxq2 and anterior head patterning genes II. Anterior is up. Expression is visualized by DISH, using TSA-Dylight550 (red) and NBT/BCIP (blue). Co-expression is indicated with dashed lines. $\left(\mathbf{A}_{0-6}\right) T c-r x$ is not co-expressed with $T c$-foxq2 until fully elongated germ band stages $\left(\mathbf{A}_{\mathbf{0}-\mathbf{4}}\right)$, except for two little spots in the neurogenic region in late elongating germ bands $\left(\mathbf{A}_{\mathbf{3}}\right)$. In retracting germ bands both genes are partially overlapping within the neurogenic region and in anterior parts of the labral buds $\left(\mathbf{A}_{5-6}\right)$. ( $\left.\mathbf{B}_{0-5}\right)$ Tc-chx expression is partially $\left(\mathbf{B}_{0}\right)$ and later almost completely $\left(\mathbf{B}_{1}\right)$ overlapping with $T c-f o x q 2$ expression. At later stages the co-expression is restricted to narrow stripes within the outer lateral labral and the neurogenic region $\left(\mathbf{B}_{2-3}\right)$. In early retracting germ bands $T c$-chx expression shows only a little overlap within the posterior portion of the labral buds $\left(\mathbf{B}_{4}\right)$, and at later stages an additional overlap within the neurogenic region $\left(\mathbf{B}_{5}\right) \cdot\left(\mathbf{C}_{\mathbf{0}-5}\right) T c-f k h$ shows almost no co-expression with $T c$-foxq2, except for a small domain in the stomodeal region in early retracting germ bands $\left(\mathbf{C}_{5}\right)$. $\left(\mathbf{D}_{\mathbf{0}-4}\right)$ $T c$-six4 is not co-expressed with $T c$-foxq2 in intermediate elongating germ bands $\left(D_{0}\right)$. Co-expression starts in late elongating germ bands and is throughout the depicted stages restricted to a domain within the neurogenic region $\left(D_{1-4}\right)$. $\left(E_{0-1}\right) T c$-ser is partially co-expressed with $T c$-foxq2 within a small sub-region of the $A M R$ at elongating germ band stages $\left(E_{0-1}\right)$. $\left(E_{2-4}\right)$ At later stages co-expression is restricted to outer lateral parts of the labral buds.

\subsubsection{Knock-down of $T c-f o x q 2$ leads to changed expression profiles of several anterior head patterning genes}

In order to gain more insights with respect to $T c$-foxq2 function within the gene regulatory network of the anterior head, I analyzed expression profiles of the previously described genes (see section 4.1.4.1) in Tc-foxq2 knock-down embryos. Changes in their expression profile indicate a potential regulation by $T c$-foxq2. To test this, I performed $T c-f o x q 2$ pRNAi and visualized the expression pattern of these genes in the embryonic offspring (6-26 h AEL), via ISH.

Only in the cnidarian Nematostella and the deuterostome Strongylocentrotus it has been described that six 3 and foxq 2 are interacting in the gene regulatory network of the apical pole (Range and Wei, 2016; Sinigaglia et al., 2013; Wei et al., 2009). In Tribolium Tc-foxq2 ${ }^{\text {pRNAi }}$ germ rudiments $T c$-six3 expression was strongly reduced (Figure 4.10B, empty arrowhead). At later stages the labral/median expression domain was reduced in size (Figure 4.10D, F, H: empty arrowheads), but the neurogenic/lateral domains were affected even more (Figure 4.10D, F, H: arrows). 


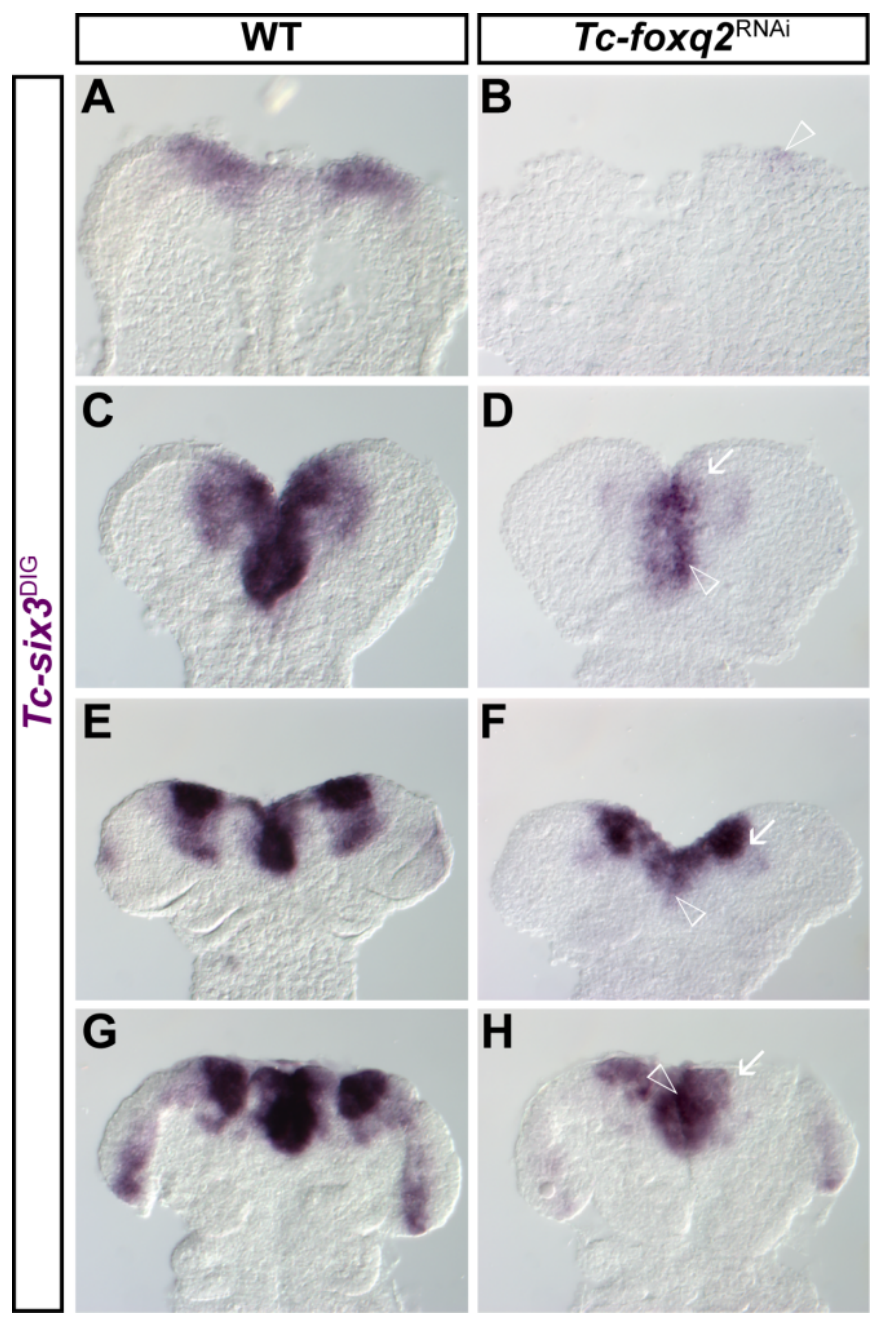

Figure 4.10 $T c$-foxq $2^{\text {pRNAi }}$ embryos show reduced $T c$-six3 expression domains. Anterior is up. Expression pattern of $T c-s i x 3$ in wt $(\mathbf{A}, \mathbf{C}, \mathbf{E}, \mathbf{G})$ and $T c-f o x q 2^{\mathrm{pRNAi}}(\mathbf{B}, \mathbf{D}, \mathbf{F}, \mathbf{H})$ embryos is monitored by ISH. (B) $T c-s i x 3$ expression is strongly reduced at $T c-f o x q 2^{\text {pRNAi }}$ germ rudiment stages (empty arrowhead). (D, F) At later stages the median (empty arrowhead) and neurogenic (arrow) Tc-six3 expression domains are reduced in size. (H) In fully elongated germ bands the labral (empty arrowhead) and the neuroectodermal (arrow) aspects of expression are strongly reduced, while the posterior median domain persists. The ocular domain appears unchanged at late stages.

The anterior median expression domain of Tc-cnc, which marks the labrum, was strongly reduced in size after knock-down of Tc-foxq2 (Figure 4.11B, D, F, H). Prior to early germ band stages no considerable changes in the expression pattern were observable. At early germ band stages the expression domain was almost completely vanished, except for an anterior remnant (Figure 4.11B, D: empty arrowhead). Subsequent stages showed predominantly reduced Tc-cnc expression within the labral buds (Figure 4.11D, F, H). 
The anterior median expression domain of $T c-c r o c$ was reduced in size throughout the analyzed stages (Figure 4.11J, L, N, P: empty arrowhead). However, the rest of the Tc-croc expression profile was not considerably affected.
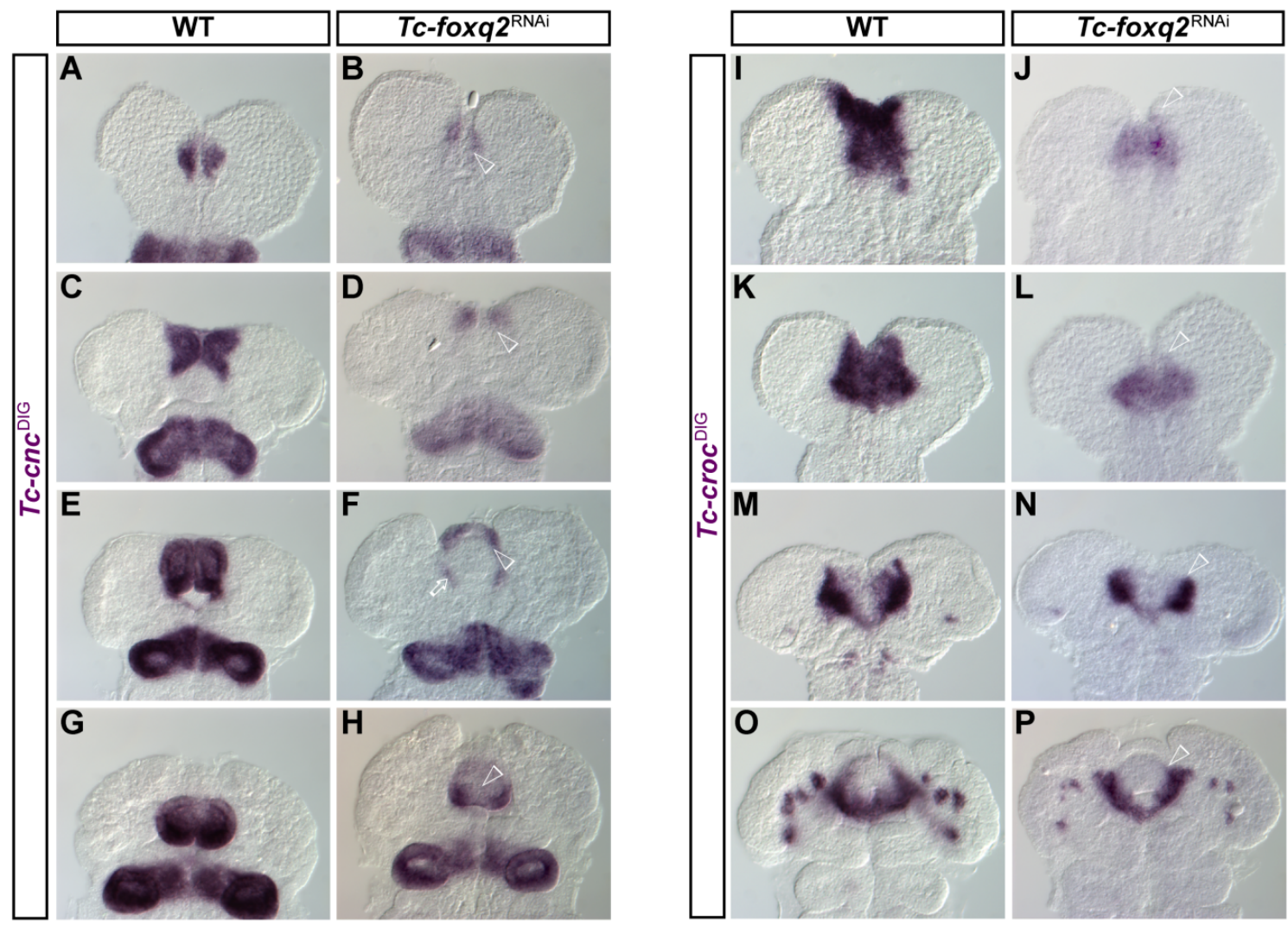

Figure 4.11 $T c$-foxq $2^{\text {pRNAi }}$ embryos show reduced $T c$-cnc and $T c$-croc expression domains. Anterior is up. Expression pattern of $T c-c n c$ in wt $(\mathbf{A}, \mathbf{C}, \mathbf{E}, \mathbf{G})$ and $T c-f o x q 2^{\mathrm{pRNAi}}(\mathbf{B}, \mathbf{D}, \mathbf{F}, \mathbf{H})$ embryos as well as expression of $T c-c r o c$ in wt (I, K, M, O) and $T c$-foxq $2^{\text {pRNAi }}(\mathbf{J}, \mathbf{L}, \mathbf{N}, \mathbf{P})$ embryos monitored by ISH. (B, D) In Tc-foxq $2^{\text {pRNAi }}$ embryos the AMR expression domain of Tc-cnc is reduced posteriorly during germ band elongation (empty arrowheads). (F) Fully elongated germ bands show reduction of expression in the labral buds as well, whereas the stomodeal expression domain appears to be only slightly decreased (empty arrow). (H) In retracting germ bands expression of $T c-c n c$ in the anterior and median region of the labral buds is strongly reduced (empty arrowhead). (J, L, N, P) Throughout the depicted stages, the Tc-croc expression pattern is lacking the anterior portion of its AMR domain (empty arrowhead).

$T c$-scro expression was laterally reduced in $T c-f o x q 2^{\mathrm{pRNAi}}$ early elongating germ bands (Figure 4.12B: empty arrowhead). In contrast to wt, Tc-foxq2 ${ }^{\text {pRNAi }}$ embryos showed a stomodeal/labral expression domain that was connected to the expression domain in the 
neurogenic tissue (Figure 4.12D, F, H: empty arrow). The ocular Tc-scro expression domains were not affected in $T c-f o x q 2^{\text {pRNAi }}$ embryos.

The expression of $T c-f k h$ was not considerably altered in $T c-f o x q 2^{\text {pRNAi }}$ embryos (Figure 4.12J, L, N, P).
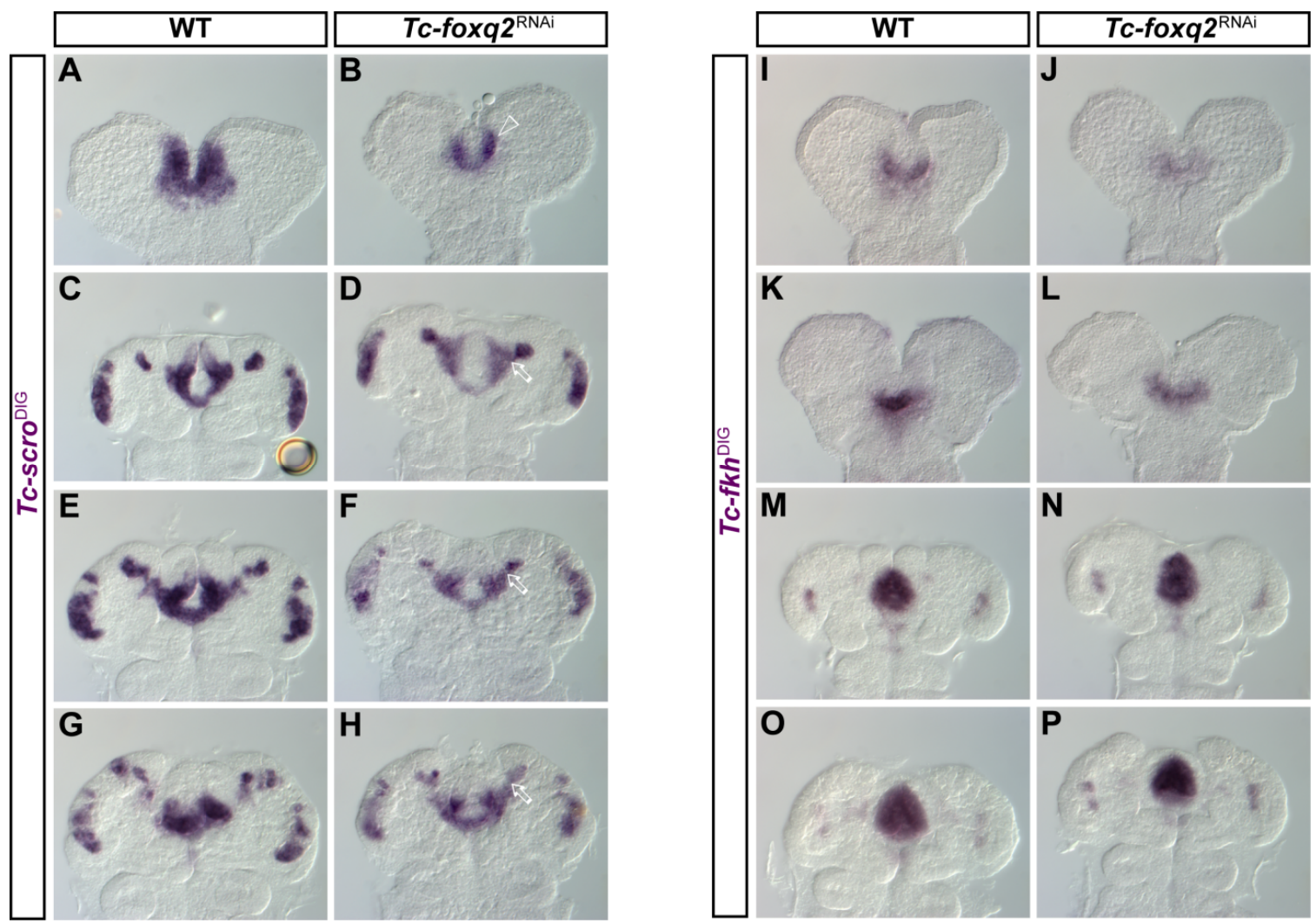

Figure 4.12 $T c$-foxq2 ${ }^{\text {pRNAi }}$ embryos show slightly altered $T c$-scro and $T c$-fkh expression profiles. Anterior is up. Expression pattern of $T c$-scro in wt $(\mathbf{A}, \mathbf{C}, \mathbf{E}, \mathbf{G})$ and $T c-f o x q 2^{\text {pRNAi }}(\mathbf{B}, \mathbf{D}, \mathbf{F}, \mathbf{H})$ embryos as well as expression of $T c-f k h$ in wt $(\mathbf{I}, \mathbf{K}, \mathbf{M}, \mathbf{O})$ and $T c-f o x q 2^{\text {pRNAi }}(\mathbf{J}, \mathbf{L}, \mathbf{N}, \mathbf{P})$ embryos monitored by ISH. (B, D, F, H) Expression of $T C$-scro is reduced to a narrow strip residing along the anterior fold (empty arrowhead) in $T c-f o x q 2^{\text {pRNAi }}$ embryos. Later stages show an atypical bridging between the labral/stomodeal and the neurogenic Tc-scro expression domains (empty arrows). (J, L, N, P) Tc-fkh expression is not considerably altered after Tc-foxq2 pRNAi.

$T c-c h x$ expression was completely lost in early elongating $T c-f o x q 2^{\text {pRNAi }}$ germ bands (Figure 4.13B: empty arrowhead). At later stages the expression domains in the labral buds (Figure 4.13D, F, H: empty arrowheads) and in the neurogenic region were reduced to a high degree (Figure 4.13D, F, H: arrow). 
The $T c$-six4 neuroectodermal expression domain was highly reduced in early $T c-f o x q 2^{\text {pRNAi }}$ embryos, showing only small expression spots at the anterior rim (Figure 4.13J: arrow). Later embryonic stages showed a reduction of the inner lateral part of the Tc-six4 expression domains (Figure 4.13L, N, P: arrows).
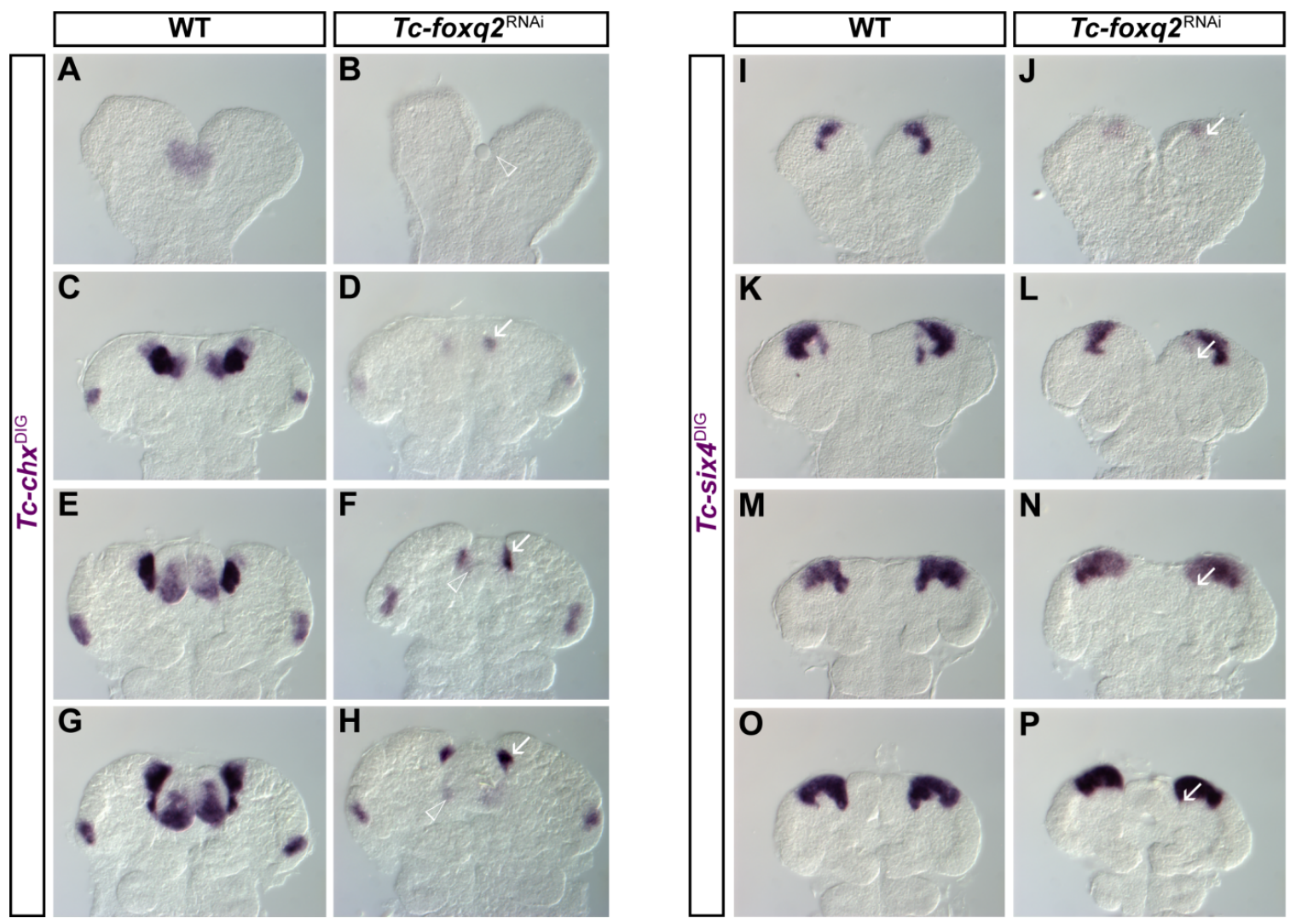

Figure 4.13 $T c$-foxq $2^{\text {pRNAi }}$ embryos show reduced $T c$-chx and $T c$-six4 expression domains. Anterior is up. Expression pattern of $T c-c h x$ in wt $(\mathbf{A}, \mathbf{C}, \mathbf{E}, \mathbf{G})$ and $T c-f o x q 2^{\text {pRNAi }}(\mathbf{B}, \mathbf{D}, \mathbf{F}, \mathbf{H})$ embryos as well as expression of $T c$-six4 in wt (I, K, M, O) and Tc-foxq2 $2^{\text {pRNAi }}(\mathbf{J}, \mathbf{L}, \mathbf{N}, \mathbf{P})$ embryos monitored by ISH. (B) Tc-chx expression is completely absent in early elongating $T c-f o x q 2^{\text {pRNAi }}$ germ bands (empty arrowhead). (D-H) At later stages the labral Tc-chx expression domains are strongly reduced (empty arrowheads) as well as the anterior neurogenic expression domains, which show only a small leftover adjacent to the labral region (arrows). The ocular Tc-chx expression domains remain unaffected. (J) Expression of Tc-six4 is strongly reduced in early elongating germ bands (arrow). (L, N, P) At later stages the inner postero-lateral parts of the neurogenic Tc-six4 expression domains are reduced (arrows).

$T c-r x$ expression at early elongating germ band stages was either reduced or completely absent after Tc-foxq2 pRNAi (Figure 4.14B). However, at later stages only the labral expression domain 
of Tc-rx was reduced or lost (Figure 4.14D, F: empty arrowheads), but the neuroectodermal domains showed no considerable changes.

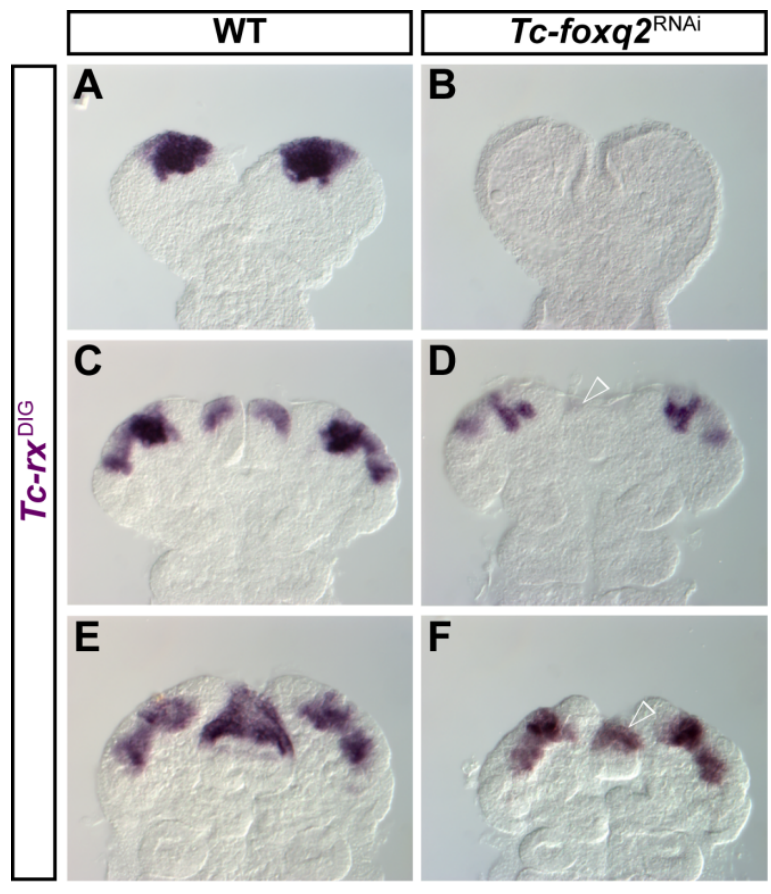

Figure 4.14 $T c$-foxq2 $2^{\text {pRNAi }}$ embryos show reduced Tc-rx expression domains. Anterior is up. Expression of $T c-r x$ in wt $(\mathbf{A}, \mathbf{C}, \mathbf{E})$ and $T c-f o x q 2^{\mathrm{pRNAi}}$ (B, D, F) embryos is monitored by ISH. (B) TC-rX expression is strongly reduced or completely absent in early elongating Tc-foxq2 $2^{\text {pRNAi }}$ germ bands. (D, F) At later stages the neurogenic Tc-rx expression pattern appears unaffected, but the labral expression domains are absent (D: empty arrowhead) or reduced in size (F: empty arrowhead).

$T c-f o x q 2^{\text {pRNAi }}$ embryos showed strongly reduced labral $T c$-wg expression domains (Figure 4.15B, D: empty arrowheads). The other segmental Tc-wg expression domains were not considerably changed.

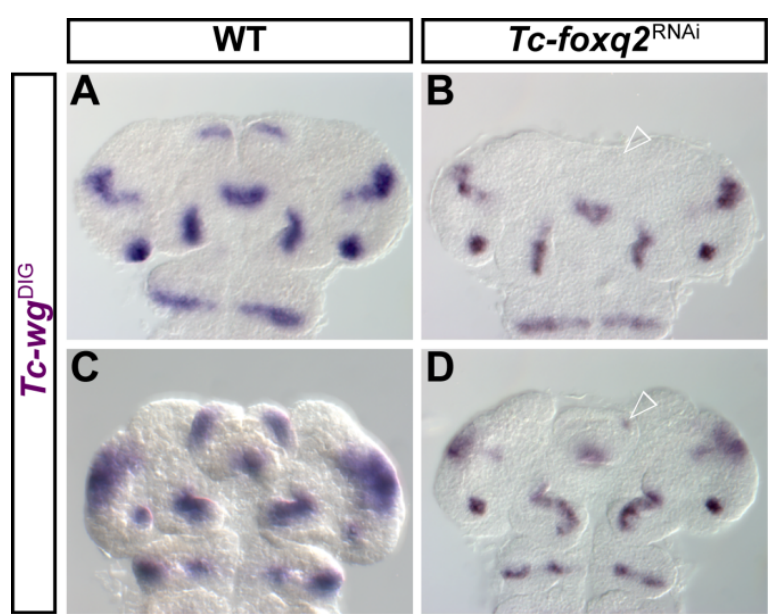

Figure 4.15 Tc-foxq2 $2^{\text {pRNAi }}$ embryos show a reduction of the labral Tc-wg expression domains. Anterior is up. Expression of $T c-w g$ in wt $(\mathbf{A}, \mathbf{C})$ and $T c$-foxq $2^{\text {pRNAi }}(\mathbf{B}, \mathbf{C})$ embryos is monitored by ISH. (B, D) TC-wg expression within the labral region is completely absent (B: empty arrowhead) or strongly reduced (D: empty arrowhead), after Tc-foxq2 pRNAi. 
The expression profiles of Tc-arr, Tc-ser and Tc-mib1 were not different comparing wt and Tc-foxq2 ${ }^{\text {pRNAi }}$ embryos (data not shown).

Taken together, $T c$-foxq2 knock-down caused altered expression of several genes, which are involved in patterning of the AMR and the adjacent neurogenic region. Considering the unchanged cell death rate at these early stages and the co-expression analysis, most of the early effects of $T c-f o x q 2$ on these genes are presumably primary effects on gene regulation. However, the deleted expression of $T c-r x$ at early embryonic stages (Figure 4.14B) is an exceptional case, because of the lack of co-expression with $T c-f o x q 2$ at this stage (Figure $4.9 \mathrm{~A}_{0-2}$ ). This observation points to secondary effects, which could be responsible for the loss of the Tc-rx expression domain.

\subsubsection{Knock-down of anterior head patterning genes altered Tc-foxq2 expression}

\section{profile}

To get hints regarding the regulation of $T c$-foxq 2 by other factors, within the anterior head gene regulatory network, I knocked-down the anterior head patterning genes and looked for altered $T c$-foxq 2 expression. Alterations in the $T c-f o x q 2$ expression profile would point to a potential regulation of Tc-foxq2 by these factors. To this end, I performed pRNAi of the head patterning genes and visualized the expression pattern of $T c-f o x q 2$ in embryonic offspring (6-26 h AEL) via ISH.

In $T c$-six $3^{\text {pRNAi }}$ embryos Tc-foxq2 expression was completely vanished (Figure 4.16B, D, F, H), except for the posterior portion of the stomodeal $T c-f o x q 2$ expression domain in retracting germ bands (Figure 4.16J). The loss of $T c$-foxq2 expression was most likely due to a mixture of real genetic interaction and secondary effects, caused by a loss of neurogenic and anterior median tissue in Tc-six3 ${ }^{\text {pRNAi }}$ embryos (Kittelmann, 2012; Posnien et al., 2011b). Based on the finding, that loss of tissue after Tc-six3 pRNAi starts in elongating germ bands (Kittelmann, 2012), the altered $T c-f o x q 2$ expression was, at earlier stages, most likely caused by primary interactions.

In early elongating $T c-c r o c^{\text {pRNAi }}$ germ bands the bilaterally expressed $T c$-foxq2 domains were expanded towards the posterior pole and fused at the embryonic midline (Figure 4.16L). Earlier stages showed no considerable expression alteration. At late elongating germ band stages, the Tc-foxq 2 expression pattern was slightly misarranged and fused at the embryonic midline as well (Figure 4.16N). Following stages showed a deletion of the stomodeal domain (Figure 4.16P, R: 
empty arrowheads) and an altered arrangement of the $T c$-foxq 2 expression domains presumably caused by the previously described misplacement of the labrum (Kittelmann et al., 2013; Figure 4.16P, R: dashed arrows).
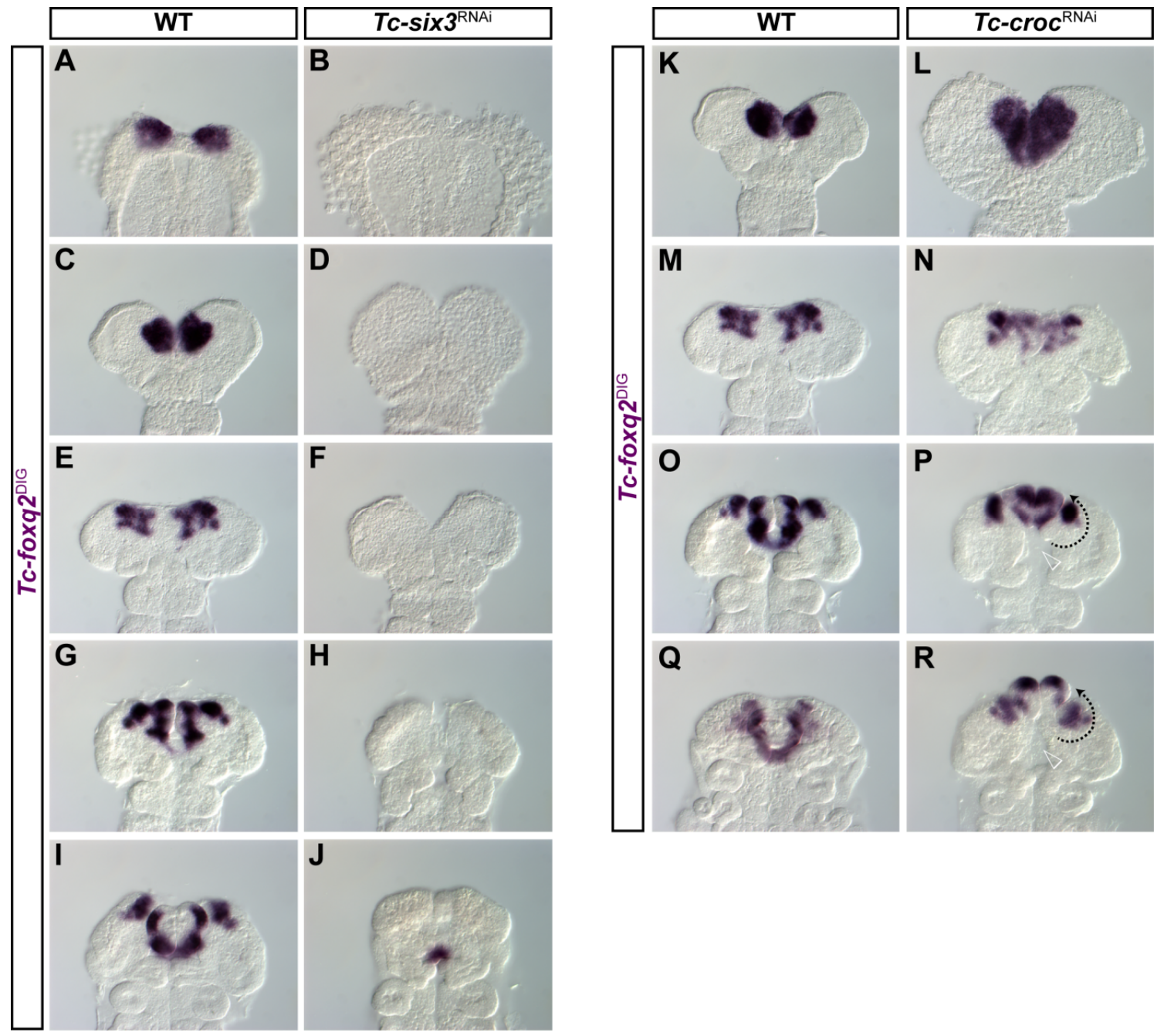

Q

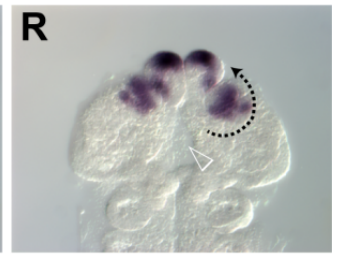

Figure 4.16 $T c$-six ${ }^{\text {pRNAi }}$ and $T c$-croc ${ }^{\text {pRNAi }}$ embryos show altered $T c$-foxq2 expression profiles. Anterior is up. Expression of $T c$-foxq2 in wt (A, C, E, G, I, K, M, O, Q), Tc-six $3^{\text {pRNAi }}(\mathbf{B}, \mathbf{D}, \mathbf{F}, \mathbf{H}, \mathbf{J})$, and $T c-\operatorname{croc}^{\text {pRNAi }}(\mathbf{L}, \mathbf{N}, \mathbf{P}$, R) embryos is monitored by ISH. (B, D, F, H) In $T c$-six $3^{\text {pRNAi }}$ embryos the $T c$-foxq2 expression is completely abolished. (J) Only the posterior part of the stomodeal $T c$-foxq2 domain remains in retracting germ bands after Tc-six3 pRNAi. Loss of $T c$-foxq2 is most likely due to a combination of primary effects and loss of anterior tissue after $T c$-six 3 pRNAi. (L) In early elongating germ bands $T c-c r o c^{\text {pRNAi }}$ embryos show a posterior expansion of $T c-f o x q 2$ expression. The embryo appears larger due to preparation. (N) Late elongating embryos show a reduced and disarranged expression pattern. $(\mathbf{P}, \mathbf{R})$ Later stages show a postero-medial deletion of $T c$-foxq2 expression (empty arrowheads). Additionally, the composition of the $T c-f o x q 2$ expression is altered due to a misplacement of the labral buds (indicated by dashed arrows). 
The expression domains of $T c-f o x q 2$ at early embryonic stages were expanded laterally after Tc-arr pRNAi (Figure 4.17B, D, F). In late elongating and fully elongated Tc-arr ${ }^{\text {pRNAi }}$ embryos appear the neurogenic expression domains expanded towards posterior (Figure 4.17H, J: arrows), whereas the labral expression domains are reduced anteriorly (Figure 4.17H, J: empty arrowheads). I assume that the early effects are genetic effects due to an ectopic de-repression, because the head morphology showed no dramatic changes (Figure 4.17B, D). Also the later changes in expression pattern are presumably due to genetic effects may as well as secondary effects due to tissue loss (Figure 4.17F, H, J).

In early and intermediate elongating $T c-m i b 1^{\text {pRNAi }}$ embryos the $T c-f o x q 2$ expression domains appear laterally decreased in size (Figure 4.17L, N: empty arrowheads). Prior to this stage expression was not altered considerably. At following stages the neurogenic Tc-foxq 2 expression domains (Figure 4.17P, $\mathrm{T}$ : arrows) as well as the anterior portion of the labral bud expression domain were reduced (Figure 4.17P, R, T: empty arrowheads). Disturbance of Tc-foxq2 expression caused by Tc-mib1 pRNAi is most likely based on a combination of genetic interaction and secondary effects. The early defects are considered being primary effects. However, for these stages it is unknown whether head tissue is lost after Tc-mib1 pRNAi treatment. Loss of the $T c$-foxq2 expression domain in the labral bud is due to the absence of the labral tissue caused by decreased cell proliferation rates (Siemanowski et al., 2015). The changes in the neuroectoderm are considered as genetic interaction of $T c-m i b 1$ on $T c-f o x q 2$, because the tissue of this region is not affected upon Tc-mib1 pRNAi (Siemanowski et al., 2015). 

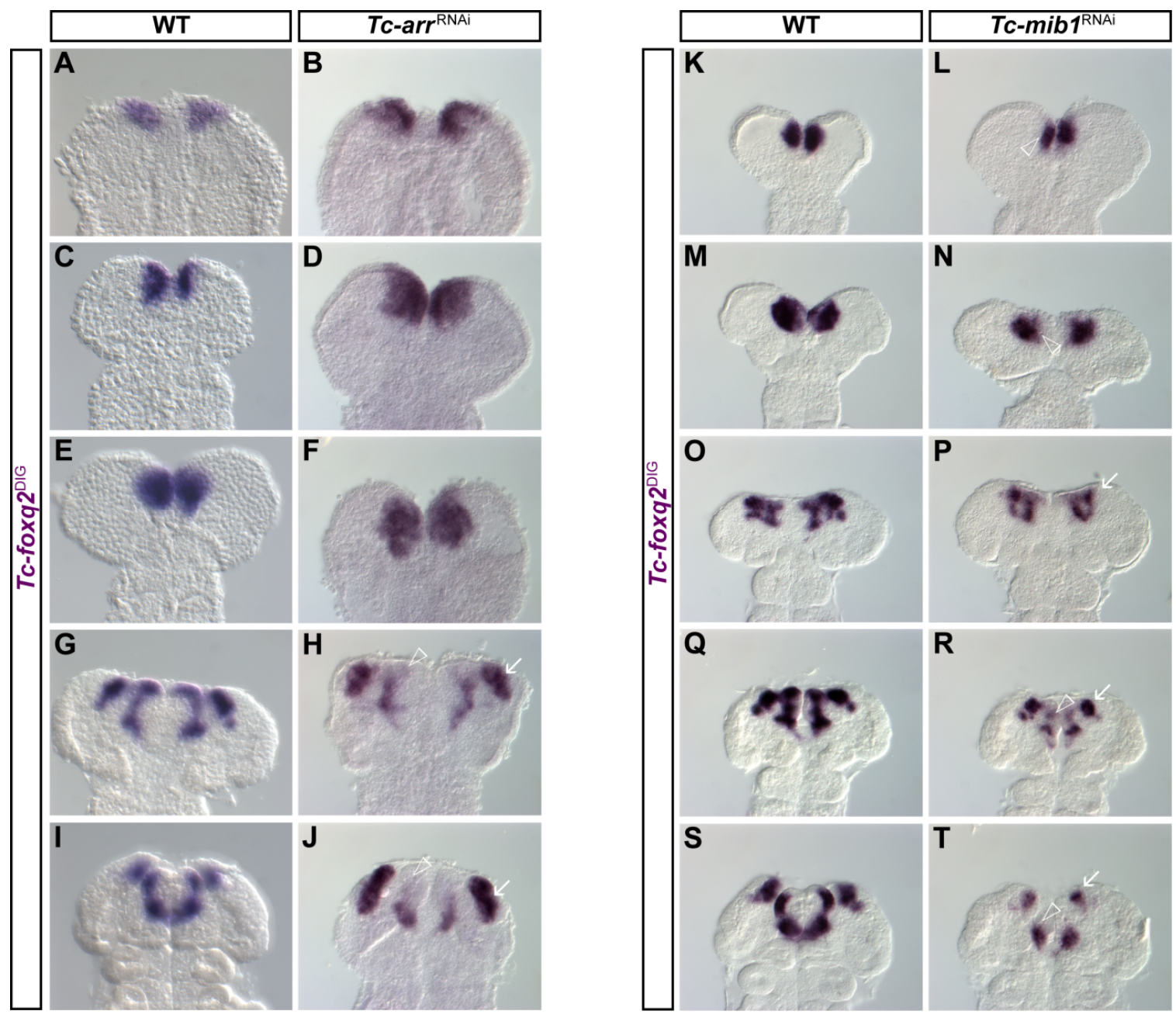

Figure 4.17 $T c$ - $a r r^{\mathrm{pRNAi}}$ and $T c-$ mib $^{\mathrm{pRNAi}}$ embryos show altered $T c$-foxq2 expression profiles. Anterior is up. Expression of $T c-f o x q 2$ in wt $(\mathbf{A}, \mathbf{C}, \mathbf{E}, \mathbf{G}, \mathbf{I}, \mathbf{K}, \mathbf{M}, \mathbf{O}, \mathbf{Q}, \mathbf{S}), T c-a r r^{\mathrm{pRNAi}}(\mathbf{B}, \mathbf{D}, \mathbf{F}, \mathbf{H}, \mathbf{J})$, and $T c-m i b 1^{\mathrm{pRNAi}}(\mathbf{L}, \mathbf{N}$, $\mathbf{P}, \mathbf{R}, \mathbf{T})$ embryos monitored by ISH. (B, D, F) Early elongating $T c-a r r^{\mathrm{pRNAi}}$ embryos show expanded $T c-f o x q 2$ expression domains. $(\mathbf{H}, \mathbf{J})$ At later stages, the neurogenic $T c$-foxq2 expression domains are posteriorly expanded (arrows), whereas the anterior portion of the labral bud expression domain is deleted (empty arrowhead). (L, N) Early elongating $T c-m i b 1^{\text {pRNAi }}$ embryos show medially reduced $T c-f o x q 2$ expression domains (empty arrowhead). (P) In late elongating $T c-m i b 1^{\text {pRNAi }}$ embryos is the neurogenic part of the Tc-foxq2 expression reduced (arrow). (R, T) Later stages show a reduction of the neurogenic expression domains and a reduction of the anterior portion of the labral bud domains (empty arrowhead). The late effect, in the labral buds $(\mathbf{R}, \mathbf{T})$, is most likely due to a loss of tissue caused by Tc-mib1 pRNAi.

Late $T c-s c r O^{\text {pRNAi }}$ germ band stages showed a reduced and misarranged Tc-foxq2 expression pattern (Figure 4.18B, D: empty arrowheads). Earlier stages showed no considerable alteration of the expression profile. In retracting $T c-s c r o^{\text {pRNAi }}$ embryos the $T c$-foxq 2 expression domain 
within the labral buds seemed to be altered in its shape and in its composition, caused by malformation of the labrum (Posnien et al., 2011b; Figure 4.18F: white arrowhead).

$T c-c n c^{\text {pRNAi }}$ embryos showed a complete loss of the $T c$-foxq2 expression domains within the labral buds (Figure 4.18H, J, L: arrowheads). This loss of Tc-foxq2 expression is due to the absence of labral tissue, caused by Tc-cnc pRNAi (Kittelmann et al., 2013). Prior to late elongating germ band stages expression was not altered considerably.
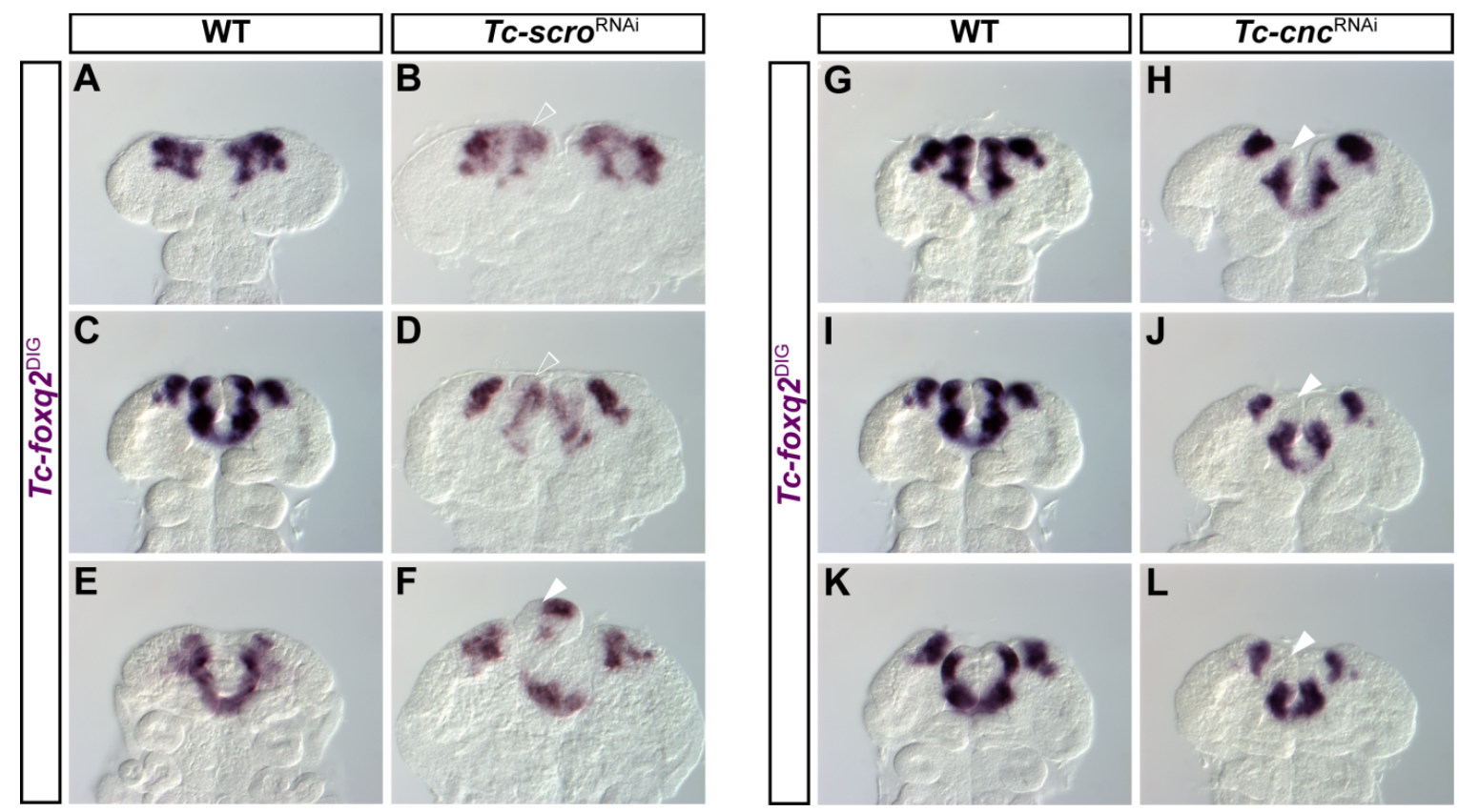

Figure 4.18 $T c$-scro ${ }^{\text {pRNAi }}$ and $T c-c n c^{\text {pRNAi }}$ lead to a changed $T c$-foxq2 expression profile at late stages only. Anterior is up. Expression of $T c-f o x q 2$ in wt $(\mathbf{A}, \mathbf{C}, \mathbf{E}, \mathbf{G}, \mathbf{I}, \mathbf{K}), T c-s c r 0^{\text {pRNAi }}(\mathbf{B}, \mathbf{D}, \mathbf{F})$, and $T c-c n c^{\text {pRNAi }}(\mathbf{H}, \mathbf{J}, \mathbf{L})$ embryos is monitored by ISH. (B) Tc-scro ${ }^{\text {pRNAi }}$ embryos show a slightly distorted $T c$-foxq2 expression pattern at late elongating germ band stages within the prospective labral/stomodeal region (empty arrowhead). (D) Fully elongated germ bands show a residual labral Tc-foxq2 expression domain, which appears to be misplaced (empty arrowhead). (F) Retracting $T c$-scro ${ }^{\text {pRNAi }}$ germ bands show a $T c$-foxq2 expression that appears to be altered mainly due to a disarrangement of the labral buds (arrowhead). $(\mathbf{H}, \mathbf{J}, \mathbf{L})$ Late $T c-c n c^{\text {pRNAi }}$ embryos show no $T c-f o x q 2$ expression within the labral buds, caused by an RNAiinduced loss of tissue (arrowhead).

Embryos prior to late elongating germ band stages showed no considerable expression alteration. $T c$-six $4^{\text {PRNAi }}$ embryos appear to show a slight reduction of the $T c$-foxq2 expression domains within the neurogenic region (Figure 4.19B, D, F, H, J: arrows). It is known that 
Tc-six $4^{\text {PRNAi }}$ leads to slight malformations of the anterior L1 larval head (Posnien et al., 2011a), however the embryonic origin of this phenotype is unknown. Based on the overall intact head morphology, this effect is unlikely to be secondary.

Also $T c-c h x^{\text {pRNAi }}$ had only an effect on the neurogenic $T c-f o x q 2$ expression domains, from late elongating germ band stages onwards. However, in this case the neurogenic expression domain was expanded, which resulted in a bridging between the neurogenic and the stomodeal $T c-f o x q 2$ expression domains (Figure 4.19L, N, P: arrows). The observed effect was most likely due to a de-repression of $T c$-foxq 2 activity, after $T c-c h x^{\text {pRNAi }}$. Secondary effects are less likely due to the fact that Tc-chx pRNAi is not associated with epidermal defects and causes only a loss of the central body in the L1 larval brain (Koniszewski, 2011).

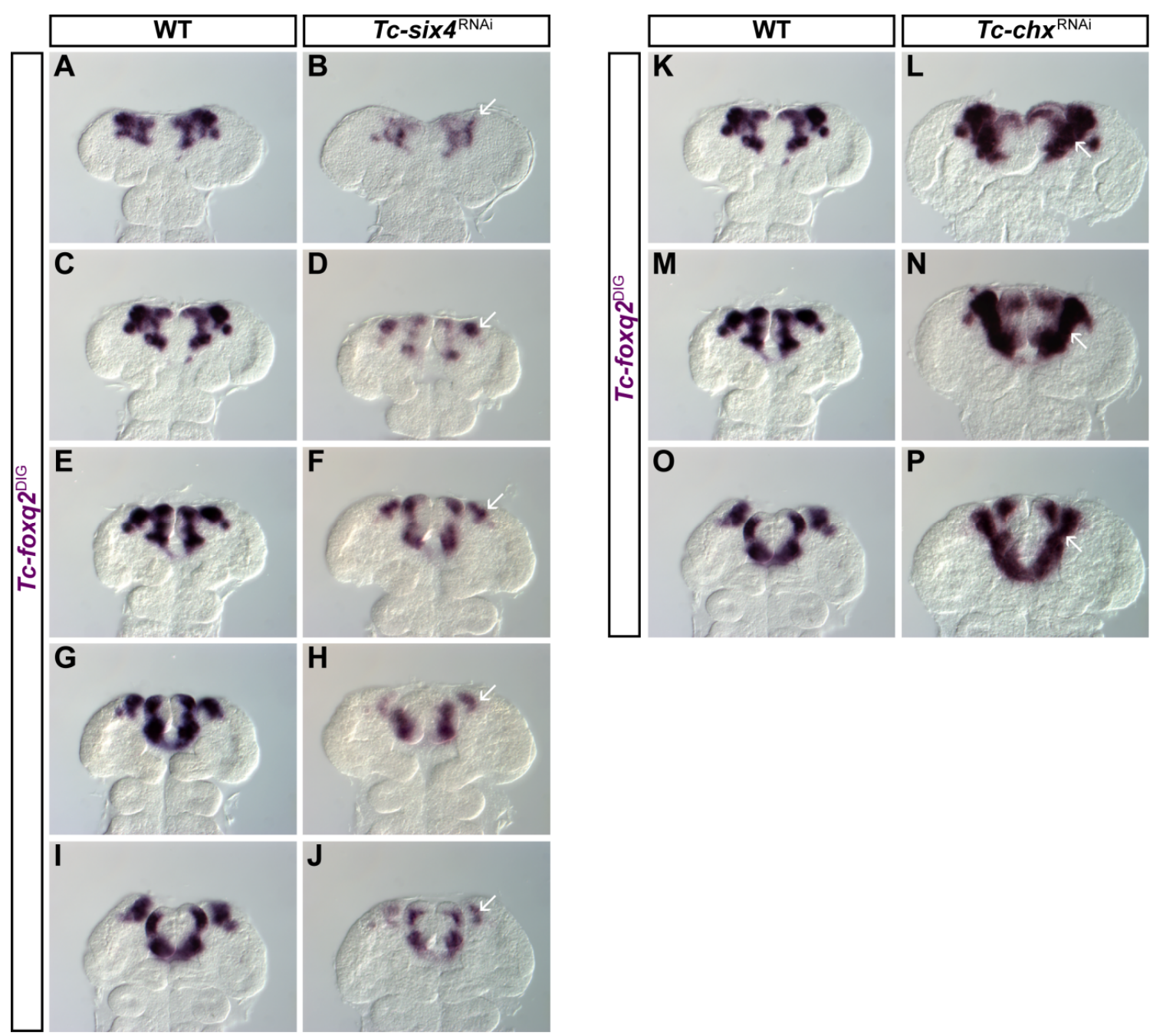


Figure 4.19 $T c-s i x 4^{\text {pRNAi }}$ and $T c$ - $c h x^{\text {pRNAi }}$ embryos show an altered $T c$-foxq2 expression profile at late stages. Anterior is up. Expression of $T c-f o x q 2$ in wt $(\mathbf{A}, \mathbf{C}, \mathbf{E}, \mathbf{G}, \mathbf{I}, \mathbf{K}, \mathbf{M}, \mathbf{O}), T c-s i x 4^{\mathrm{pRNAi}}(\mathbf{B}, \mathbf{D}, \mathbf{F}, \mathbf{H}, \mathbf{J})$, and $T c-c h x^{\text {pRNAi }}(\mathbf{L}, \mathbf{N}, \mathbf{P})$ embryos is monitored by ISH. (B, D, F, H, J) Tc-six4 ${ }^{\text {pRNAi }}$ embryos show a reduction of the neurogenic $T c$-foxq2 expression domains (arrows). (L, N, P) Late elongating to retracting $T c$ - $c h x^{\text {pRNAi }}$ germ bands show an expansion of the neurogenic $T c$-foxq2 expression domains (arrows), which leads to a fusion with the stomodeal expression domain.

The expression pattern of $T c-f o x q 2$ at late elongating $T c-f k h^{\text {pRNAi }}$ germ band stages was misarranged, caused by a turning-in of the head lobes towards the midline (Figure 4.20B, C, E, F). The misarranged $T c-f o x q 2$ expression domains in $T c-f k h^{\text {pRNAi }}$ embryos were most likely caused by a malformation of the tissue. Although it has been reported for $T c-f k h$ to have only an embryonic function in hindgut development (Schoppmeier and Schröder, 2005) the embryos appeared to have also defects in the stomodeal tissue.

$T c-r x^{\mathrm{pRNAi}}$ embryos showed no considerable change of the Tc-foxq2 expression pattern (Figure 4.20H, J, L).
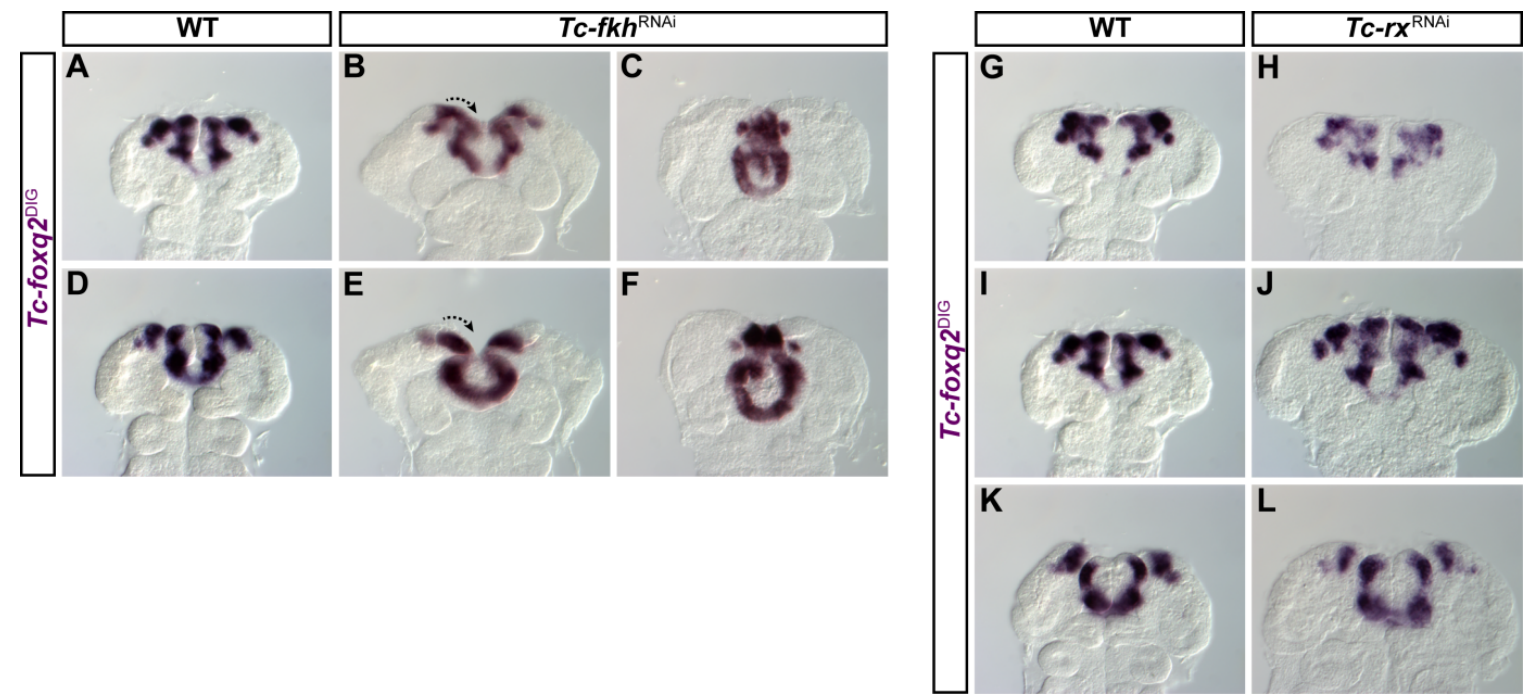

Figure 4.20 $T c-f k h^{\text {pRNAi }}$ embryos show a secondary alteration of $T c$-foxq2 expression, whereas $T c-r x^{\text {pRNAi }}$ embryos show no considerable change in Tc-foxq2 expression profile. Anterior is up. Expression of $T c$ foxq2 in wt (A, D, G, I, K), Tc-fk $h^{\text {pRNAi }}(\mathbf{B}, \mathbf{C}, \mathbf{E}, \mathbf{F})$ and $T c-r x^{\mathrm{pRNAi}}(\mathbf{H}, \mathbf{J}, \mathbf{L})$ embryos is monitored by ISH. (B, C, E, F) Fully elongated $T c-f k h^{\text {pRNAi }}$ embryos show an altered $T c$-foxq2 expression pattern. Change of the expression pattern is probably a secondary effect and caused by an intermediate (B, E) or strong (C, F) turning of the antero-lateral head tissue towards the embryonic midline (B, E: turning indicated by dashed arrows). (H, J, L) In Tc-rx ${ }^{\text {pRNAi }}$ embryos no considerable change of the $T c$-foxq2 expression pattern is detectable. 


\subsubsection{Tc-foxq2 gain-of-function analysis}

The knock-down experiments provided already some information about the function of Tc-foxq2 and its role within the gene regulatory network of the anterior pre-segmental head. In order to substantiate these findings and to gain more information about $T c-f o x q 2$, I exploited the heat shock system to drive ectopic Tc-foxq2 expression (Lindquist, 1986; Schinko et al., 2012). To this end, I generated a construct, which ubiquitously drives the expression of $T c$-foxq2 (full coding sequence) under the control of the Tc-heat shock protein 68 promoter (hsp68; Figure 4.21).

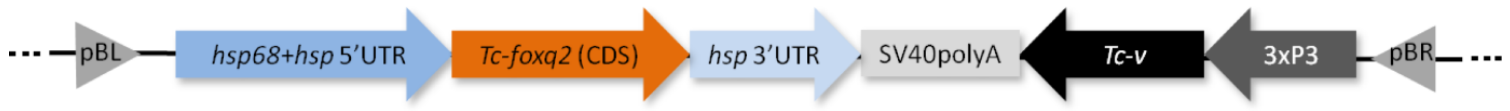

Figure 4.21 Transgenesis construct for heat shock-inducible $T c$-foxq2 misexpression lines. $p B L$ and $p B R$ : piggyBac sites for transposon based integration, hsp68+hsp 5'UTR: heat shock protein 68 promoter + heat shock protein $5^{\prime}$ untranslated region, $T c$-foxq2 (CDS): full coding sequence of $T c-f o x q 2$, hsp 3'UTR: heat shock protein 3' untranslated region, SV40polyA: polyadenylation signal, Tc-v: Tc-vermillion (Transgenesis marker), 3xP3: eye-specific promoter.

\subsubsection{Ectopic expression of Tc-foxq2 - Proof of principle}

In order to test whether the generated transgenic lines were functional, I performed a heat shock treatment with embryos (0-24 h AEL) of all independent transgenic lines, fixated them after $30 \mathrm{~min}$, and visualized the Tc-foxq2 expression via ISH. This control experiment showed that all independent transgenic lines $(n=8)$ were functional, but the degree of ectopic expression was highly variable. Some lines showed a very patchy expression pattern, with only a few Tcfoxq2 expressing cells (Figure 4.22B-B' ${ }^{\prime \prime}$ ). In contrast, other lines showed a spotty but an equally distributed Tc-foxq2 expression throughout the embryo (Figure 4.22C-D $\left.{ }^{\cdots}\right)$ ). It has to be noted that all lines showed ectopic expression, while the actual $T c$-foxq2 expression domains appeared not to be increased in their signal. For further analysis I used the transgenic line hsp68-Tc-foxq $2_{w 5}$, which showed the most even signal distribution. 


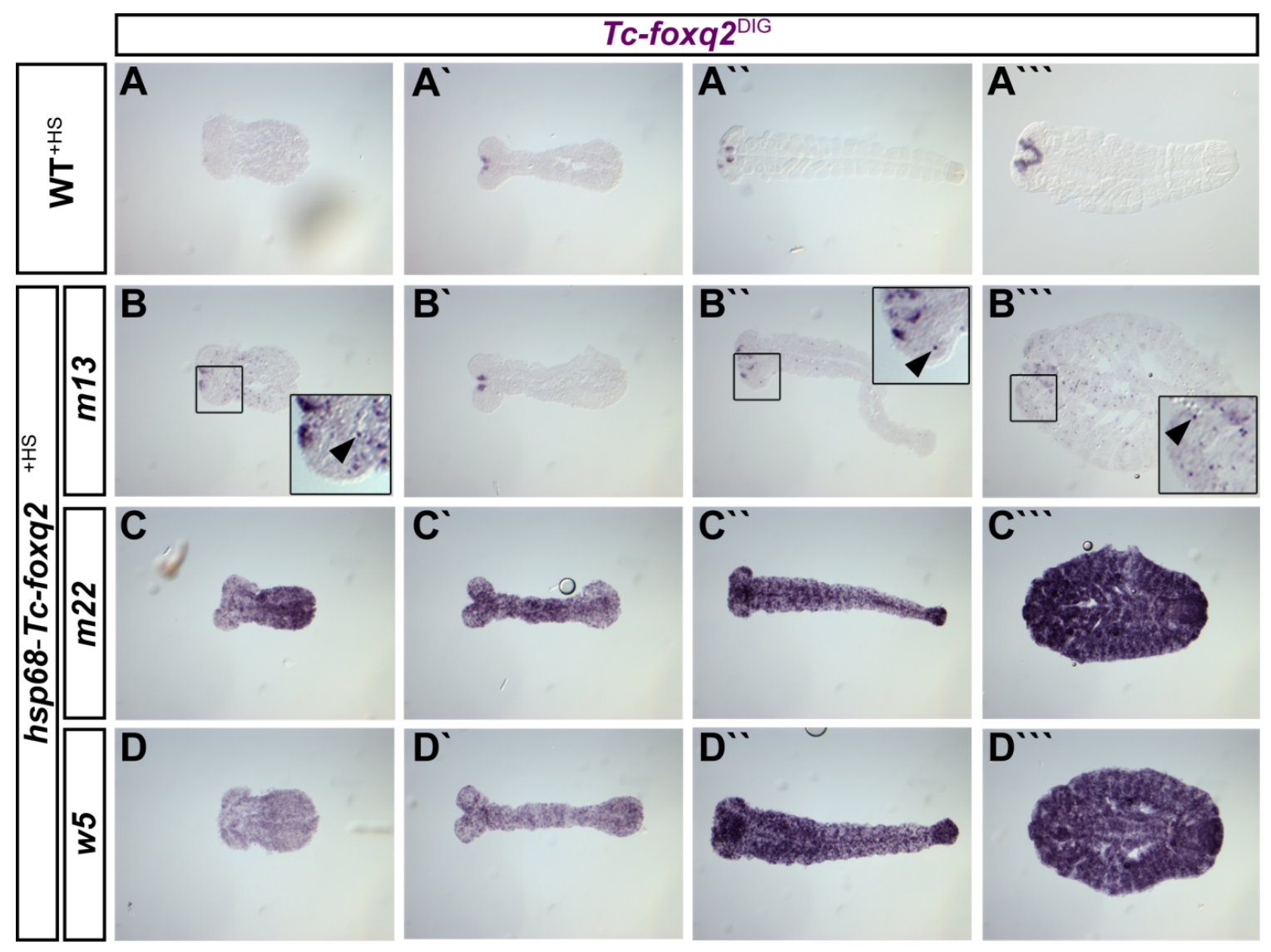

Figure 4.22 Tc-foxq2 gain-of-function lines show heat shock-induced ectopic Tc-foxq2 expression. Anterior is left. Expression of $T c-f o x q 2$ in heat shock-treated wt (A-A $\left.{ }^{\cdots}\right)$, hsp68-Tc-foxq2 ${ }_{m 13}$ (B-B $\left.{ }^{\cdots \cdot}\right)$, hsp68-Tc-foxq2 $2_{m 22}\left(\mathbf{C}^{\left.-\mathbf{C}^{\cdots}\right)}\right)$, and $h s p 68-T c-f o x q 2_{w 5}\left(\mathbf{D}-\mathbf{D}^{\cdots}\right)$ embryos is monitored by ISH. (A-A $\left.{ }^{\cdots}\right) \mathrm{Wt}$ embryos show no change in the Tc-foxq2 expression pattern upon heat shock treatment. (B-B '“) Individuals of the transgenic line $h s p 68-T c-f o x q 2_{m 13}$ show ectopic $T c$-foxq2 expression in some cells that are scattered sparsely across embryo (boxes, arrowheads). (C-D $\left.{ }^{\cdots}\right)$ Individuals of the transgenic lines $h s p 68-T c-f o x q 2_{m 22}$ and $h s p 68-T c-f o x q 2_{w 5}$ show a strong activation of ectopic Tc-foxq2 expression throughout the embryo. For the following experiments the line $h s p 68-T c-f o x q 2_{w 5}$ was used, because of the more even distribution of ectopic Tc-foxq2 expression (D-D $\left.{ }^{\cdots}\right)$.

\subsubsection{Analysis of the epidermal $T c$-foxq2 gain-of-function phenotype}

\subsection{Ectopic Tc-foxq2 expression resulted in a pleiotropic but specific epidermal L1} phenotype

To gain more insights about $T c$-foxq 2 function, I was interested in the epidermal phenotype after ectopic expression of $T c$-foxq2 at early embryonic stages. For this gain-of-function experiment, I 
collected and heat shocked embryos (0-24 h AEL) from the $h s p 68-T c$-foxq2 line. After four days, I analyzed the L1 larval cuticles.

All of the analyzed cuticles showed various defects. However, two distinct classes of phenotypes were observed consistently. The first class showed defects in all three tagmata (Figure 4.23B, $\left.B^{\prime}\right)$. The head capsule and the gnathal appendages appeared to be unaffected. However, the antennal flagellum was completely absent in almost all cuticles of this class and at times the head bristle pattern was partially disrupted (Figure 4.23B': left panel). The legs showed a reduced number of podomeres, probably lacking the femur and tibia (Figure 4.23B': mid panel). The abdomen lacked a variable number of segments and the remaining segments were dorsally fused (Figure 4.23B': right panel). The urogomphi and pygopods were frequently affected (Figure 4.23B': right panel). The second class of phenotypes showed defects that were restricted to the pre-abdominal region (Figure 4.23D, D'). Most of the cuticles showed defects in the head capsule, the head appendages, and very often defects in the head bristle pattern. (Figure 4.23DD": left panel). Notably, these defects were found in regions where Tc-foxq2 pRNAi leads to loss of structures (e.g. clypeus bristle and anterior vertex setae). The legs showed the same defects as described above (Figure 4.23D": right panel). Taken together, these results show that the phenotype is characterized by several defects affecting several epidermal structures, but that within this variation two different classes of phenotypes with specific sets of defects are recurring. The two different phenotype classes show a subdivision with the emphasis on more posterior (Class I) versus more anterior defects (Class II). This may reflect different phenotypic outcomes resulting from different timing of the ectopic $T c$-foxq2 expression. 


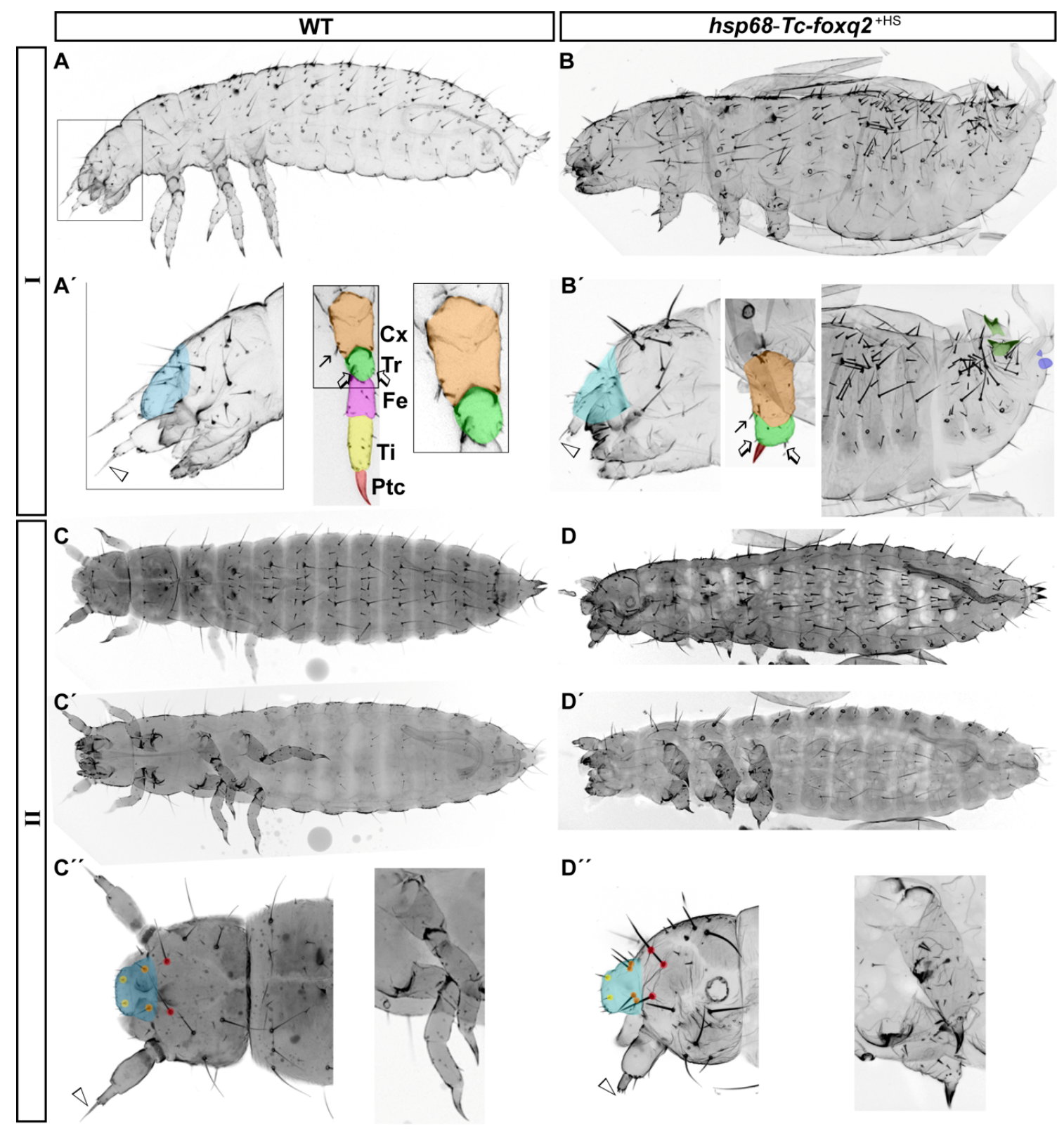

Figure 4.23 Embryonic Tc-foxq2 gain-of-function results in defects in L1 larval cuticles. Anterior is left. Wt (A, A', C-C'") and heat shock-treated hsp68-TC-foxq2 (B, B', D-D“') L1 larval cuticles, grouped into two classes (I and II). (B, B') L1 cuticles of the phenotype class I lack the antennal flagellum (B', left panel: arrowhead) and a slightly disrupted bristle pattern. The legs (middle panel) have a reduced number of podomeres. Presumably only the coxa (orange), trochanter (green) and the pre-tarsal claw (red) are left (compare bristles marked by arrows in $\mathbf{A}^{\mathbf{*}}$ and $\mathbf{B}^{\mathbf{}}$ ). Abdominal segments (right panel) are reduced in number and remaining segments are dorsally fused. The urogomphi (green, duplicated in this specimen) and pygopods (blue, reduced in this specimen) are sometimes affected. (D-D') L1 cuticles of the phenotype class II show defects restricted to the head and thoracic region. (D“) A larval head (left panel) of the phenotype class II lacking an antennal flagellum (arrowhead) and showing affected head appendages. The head bristle pattern is disrupted and frequently shows a duplication of the clypeus setae 
(orange dots) and the anterior vertex setae (red dots). The legs (right panel) show comparable defects as the legs of the first phenotype class. Cx: coxa, Tr: trochanter, Fe: femur, Ti: tibia, Ptc: pre-tarsal claw

\subsection{2.2 Epidermal phenotype - Separating late and early Tc-foxq2 function}

Based on the finding that the $T c-f o x q 2$ misexpression experiment results in distinct classes of epidermal L1 phenotypes, I tried to determine whether these differences are related to a late versus early $T c$-foxq 2 function during development. Trying to separate these aspects, I collected embryos from the $h s p 68-T c-f o x q 2$ line and grouped them in three different cohorts according to the timing of heat shock treatment (9-13 h AEL, 14-20 h AEL, and 20-25 h AEL). Subsequently, I collected and analyzed the L1 larval cuticles (exact percentages are listed in Table S7.9-.14).

The three different cohorts (1: 9-13 h AEL, germ rudiment stage in Figure $4.5 \mathrm{C}$ : stage 1; 2: $14-20 \mathrm{~h}$ AEL, elongating germ band stages in Figure 4.5C: stage 2-3; 3: $20-25 \mathrm{~h}$ AEL, fully elongated germ band stages in Figure 4.5C: stage 4-5) showed no drastic phenotypic differences (Figure 4.24A-C). Analyzing the cuticles with regard to their particular defects, it turned out that the first cohort ( $n=42$, Figure $4.24 A^{\prime}$ ) often had severe defects within the head region, showing a loss of gnathal appendages (Md: 63.1\%, Mx: 64.3\%, Lb: 76.2\%) and a disrupted head bristle pattern. This cohort also showed a high penetrance in more posterior defects (leg pair 1-3: $82.2 \%$, and abdominal segment 1-8: $48.1 \%)$. The second cohort $(n=122$, Figure 4.24B') showed minor defects in the head region, especially the gnathal appendages were only rarely affected (Md: $0.8 \%, M x: 0.8 \%, L b: 1.6 \%)$. More posterior regions were frequently affected within this cohort (Leg pair 1-3: 65.6\%, abdominal segment 1-8: 95.9\%). The last cohort ( $n=132$, Figure 4.24B') showed comparable amounts of defects in the gnathocephalic region (Md: $2.9 \%, \mathrm{Mx}$ : 4.9\%, Lb: $5.0 \%$ ), less frequent leg defects (Leg pair 1-3: 44.2\%) and less severe and frequent abdominal defects (Abdominal segment 1-8: 89.7\%). These data indicate that the timing of ectopic Tc-foxq2 expression leads to different phenotypes. Due to my interest in the head phenotype, I decided to focus on the early cohort for subsequent experiments.

In order to get an impression of the basis of the cuticle phenotype, I tested whether the phenotype resulted from an increased cell death rate. To this end, I collected eggs of the hsp68-Tc-foxq2 line and applied a heat shock treatment (9-13 h AEL), fixed the heat shocked embryos (14-18 h AEL), and performed a Dcp-1 antibody staining to mark apoptotic cells. Finally I investigated qualitative differences between wt and $T c$-foxq 2 gain-of-function embryos. Heat shock-treated wt embryos showed a slight increase in the number of apoptotic cells compared 
to untreated embryos (data not shown). Tc-foxq2 gain-of-function embryos showed a dramatically increased number of apoptotic cells when compared to heat shock-treated wt embryos (Figure 4.25A-F'). The apoptotic cells were distributed all over the embryo independent of the analyzed stage. This result suggests that the increased cell death rate is one factor for the pleiotropic larval cuticle phenotype caused by ectopic $T c$-foxq2 expression.

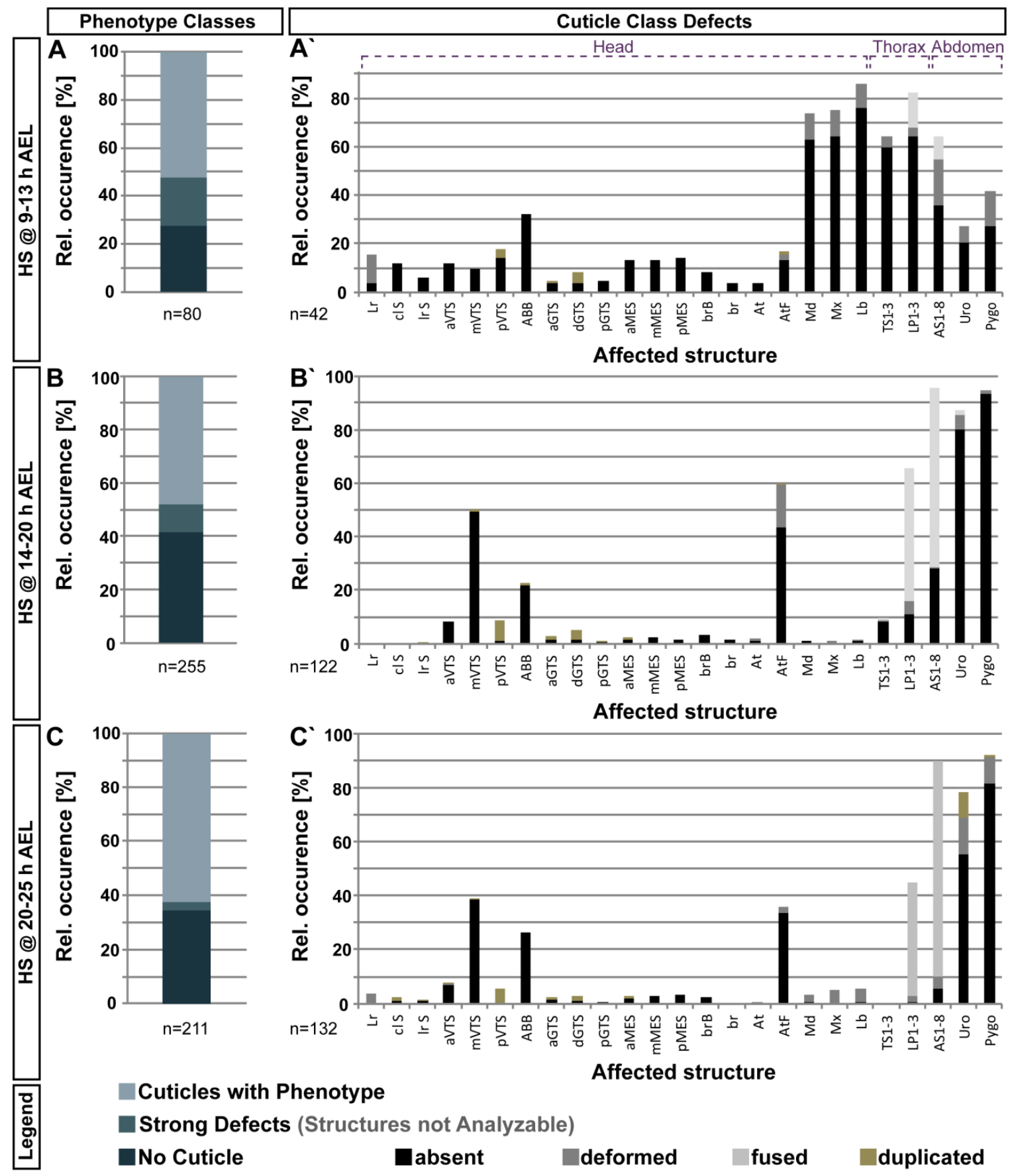


Figure 4.24 Larval epidermal defects after different onsets of embryonic Tc-foxq2 gain-of-function. Relative occurrence of eggs without cuticle (dark blue), cuticles with strong defects (blue), and cuticles with an analyzable phenotype (light blue; $\mathbf{A}-\mathbf{C}$ ) and the defects present in the 'cuticle class' $\left(\mathbf{A}^{\prime}-\mathbf{C}^{\prime}\right)$, resulting from heat shock treatment of $h s p 68-T c-f o x q 2$ embryos at different stages. (A-C) The penetrance of eggs without cuticle (no cuticle) is highest within the second cohort, where the portion of analyzable cuticles is the lowest (B). (A') Ectopic Tc-foxq2 expression in 9-13 h AEL old embryos leads to cuticles that show defects in all three body parts frequently, with the strongest defects in the gnathal appendages. (B') Heat shock-induced Tc-foxq2 gain-of-function in 14-20 h AEL old embryos leads to minor head defects, showing a loss of the antennal flagellum or a disrupted bristle pattern. The thoracic structures are frequently but mostly slightly affected. Predominant are post-cephalic defects. (C') Heat shock-induced Tc-foxq2 gain-of-function in 20-25 h AEL old embryos leads to cuticles that show the fewest and weakest defects in all three body parts. Abdominal defects are predominant. Lr: labrum, cl S: clypeus setae, Ir S: labrum setae, aVTS: anterior vertex setae, mVTS: median vertex setae, pVTS: posterior vertex setae, ABB: antenna basis bristles, aGTS: anterior gena triplet setae, dGTS: dorsal gena triplet setae, pGTS: posterior gena triplet setae, aMES: anterior maxilla escort setae, mMES: median maxilla escort setae, pMES: posterior maxilla escort setae, brB: bell row bristles, br: bell rows, At: antennae, AtF: antennal flagella, Md: mandibles, Mx: maxillae, Lb: labium, TS1-3: thoracic segments 1-3, LP1-3: leg pair 1-3, AS18: abdominal segments 1-8, Uro: urogomphi, Pygo: pygopods 


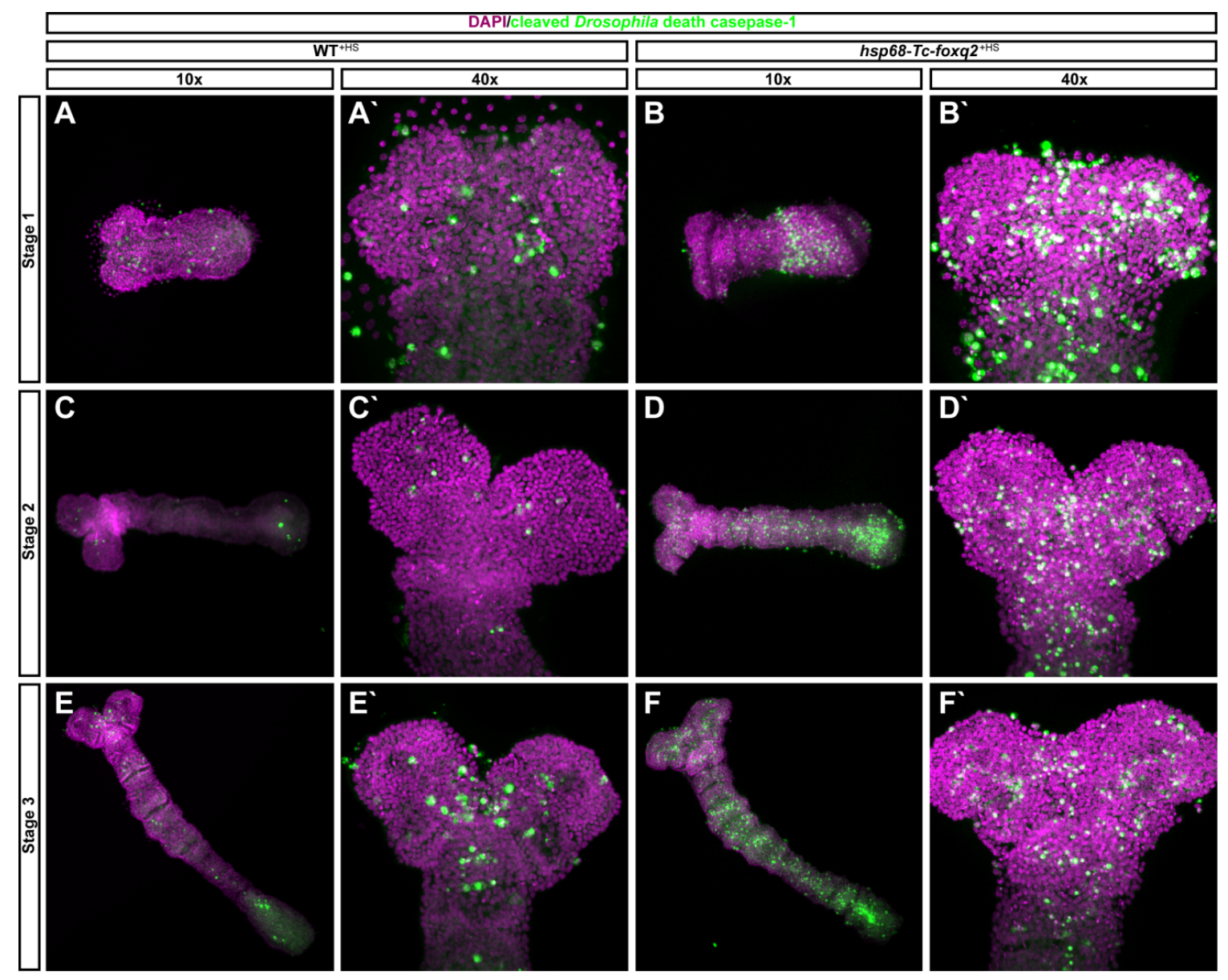

Figure 4.25 Ectopic $T c$-foxq2 expression leads to an increased number of apoptotic cells. Anterior is left in $\mathbf{A}-\mathbf{F}$ and up in $\mathbf{A}^{\prime}-\mathbf{F}^{\prime}$. Apoptotic cells of heat shock-treated wt $\left(\mathbf{A}, \mathbf{A}^{\prime}, \mathbf{C}, \mathbf{C}^{\prime}, \mathbf{E}, \mathbf{E}^{\mathbf{}}\right)$ and hsp68-Tc-foxq2 (B, $\left.\mathbf{B}^{\prime}, \mathbf{D}, \mathbf{D}^{\prime}, \mathbf{F}^{\prime}, \mathbf{F}^{\prime}\right)$ embryos are monitored by cleaved DCP-1 antibody staining (green). Nuclei are stained with DAPI (magenta) to visualize the embryonic morphology. Embryos are depicted as maximum projections. (B, B', D, D', F, F') Ectopic Tc-foxq2 expression in embryos (14-18 h AEL) leads to a strong increase in the number of apoptotic cells in all three body parts throughout the analyzed developmental stages compared to heat shocked wt embryos (B, $\left.\mathbf{B}^{\prime}, \mathbf{D}^{\prime}, \mathbf{D}^{\prime}, \mathbf{F}^{\prime}, \mathbf{F}^{\prime}\right)$.

\subsubsection{Tc-foxq2 gain-of-function and the impact on the anterior head gene regulatory}

\section{network}

With the aim to complement the picture of $T c-f o x q 2$ function in the gene regulatory network of the anteriormost head region, I analyzed $T c$-foxq 2 gain-of-function embryos for changes in expression profiles of head patterning genes. To this end, I collected embryos from the hsp68-Tc-foxq2 line, applied a heat shock treatment to the embryos (9-13 h AEL), fixed them 
(14-18 h AEL) and performed ISH to visualize potential alteration of the expression profile. The same procedure was performed simultaneously using wt embryos as control.

$T c-r x$ expression was considerably reduced to a small or spotty domain upon ectopic $T c-f o x q 2$ expression (Figure 4.26A-C). Ectopic Tc-foxq2 expression caused a premature onset of Tc-six4 expression (compare Figure 4.26D, D': younger and older with E-F'). Additionally, Tc-six4 expression domains were enlarged (Figure 4.26D: right panel and Figure 4.26E, F: arrow). Further, $h s p 68-T c-f o x q 2^{+H S}$ embryos showed novel $T c$-six4 expression domains, which seem to be located within the antennal segment (Figure 4.26E, F: white arrowhead). The Tc-scro expression pattern was altered in two different ways depending on the developmental stage (Figure 4.26GL). In $h s p 68-T c-f o x q 2^{+H S}$ germ rudiments the onset of expression Tc-scro was premature and the domains were uniformly enlarged and posteriorly elongated (Figure 4.26G-I). In contrast, elongating $h s p 68-T c-f o x q 2^{+H S}$ germ bands showed a spotty reduction of the $T c$-scro expression domain compared to the wt expression domain (Figure 4.26K, L). The effect of ectopic Tc-foxq2 expression at elongating germ band stages may be a modulated secondary effect elicited by apoptosis (see Figure 4.25). The anterior median domain of Tc-cnc expression appeared to be slightly expanded, after ectopic Tc-foxq2 expression (Figure 4.260: white empty arrowhead) and spread posteriorly (Figure 4.26N: black empty arrowhead). The Tc-cnc mandibular expression domain was reduced in a spotty manner (Figure 4.26N, O: black arrowheads). 


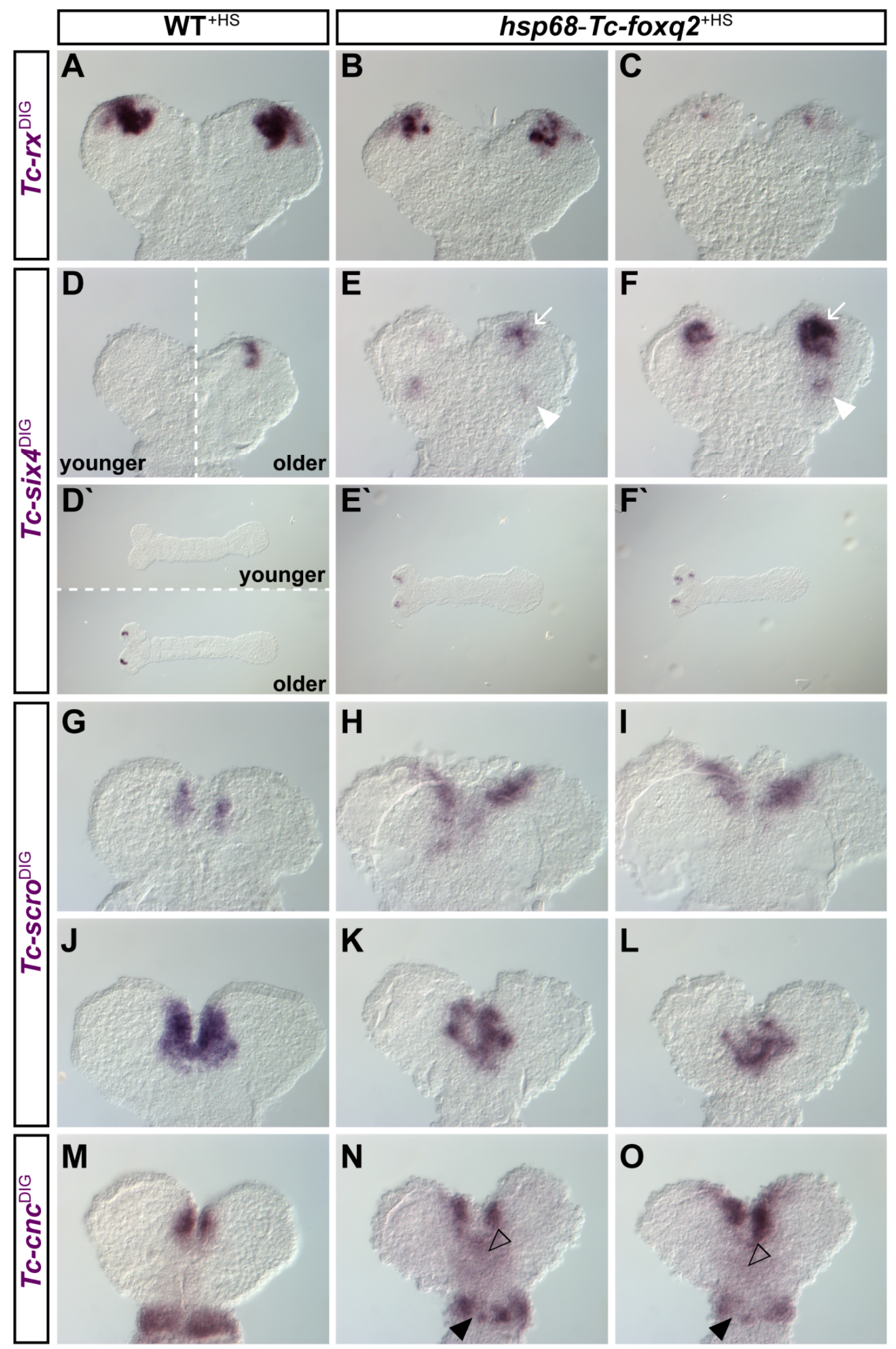


Figure 4.26 Ectopic Tc-foxq2 expression impacts head patterning gene expression profiles (strong effects). Anterior is up (left in $\mathbf{D}^{`}-\mathbf{F}^{\prime}$ ). Expression of head patterning genes in heat shock-treated wt (A, D, $\left.\mathbf{D}^{\prime}, \mathbf{G}, \mathbf{J}, \mathbf{M}\right)$ and $h s p 68-T c-f o x q 2$ (B, C, E, E', F, F', H, I, K, L, N, O) embryos (14-18 h AEL) is monitored by ISH. (B, C) Ectopic Tc-foxq2 expression leads to reduced Tc-rx expression domains (B, C). $\left(\mathbf{E}, \mathbf{E}^{\prime}, \mathbf{F}, \mathbf{F}^{\prime}\right)$ TC-six4 expression shows a premature onset (compare $\mathbf{E}^{\prime}, \mathbf{F}^{\prime}$ with $\mathbf{D}^{\prime}$ : younger and older) at the anterior tip (arrows). These premature expression domains are expanded (F: arrow) compared to the size of the wt domains. Further, hsp68-Tc-foxq $2^{+\mathrm{HS}}$ embryos show an additional Tc-six4 expression domain within the antennal segment (white arrowhead). (H, I) The Tc-scro expression domains are prematurely expressed and expanded in $h s p 68-T c-f o x q 2^{+\mathrm{HS}}$ germ rudiments. (K, L) In contrast, early elongating germ bands show reduced and aberrant $T c$-scro expression domains in $h s p 68-T c-f o x q 2^{+H S}$ embryos. (N, O) The anterior median Tc-cnc expression domains appear to be spread to the posterior ( $\mathbf{N}, \mathbf{O}$ : black empty arrowheads). The mandibular Tc-cnc expression domain is reduced in an irregular manner after ectopic Tc-foxq2 expression (black arrowheads).

$T c-w g, T c-s i x 3$, and Tc-croc showed comparably mild or less relevant alterations in expression profiles, after ectopic $T c$-foxq2 expression. $h s p 68-T c-f o x q 2^{+ \text {HS }}$ embryos showed a slight reduction of the ocular Tc-wg domain (Figure 4.27B, C: empty arrowheads). The antennal $T c-w g$ stripes were heavily reduced or completely absent in almost all hsp68-Tc-foxq2 ${ }^{\text {+HS }}$ embryos (Figure 4.27B, C: white arrowheads). The subsequent segmental expressed $T c-w g$ domains were either reduced (Figure 4.27F: black empty arrowhead), absent (Figure 4.27F: white empty arrowhead) or collapsed (Figure 4.27E: white arrowhead). This abnormal expression of the segment polarity gene $T c$-wg could relate to the cuticle phenotype, which showed deformed, absent, or fused segments (Figure 4.23). Neurogenic Tc-six3 expression was anteriorly reduced in hsp68-Tc-foxq2 $2^{\text {+HS }}$ embryos (Figure 4.27H, I: white arrows). The expression pattern of Tc-croc appears to be enlarged (Figure 4.27K, L: empty arrowheads) and spread posteriorly (Figure 4.27K, L: white arrowheads) in hsp68-Tc-foxq $2^{\text {HHS }}$ embryos. 

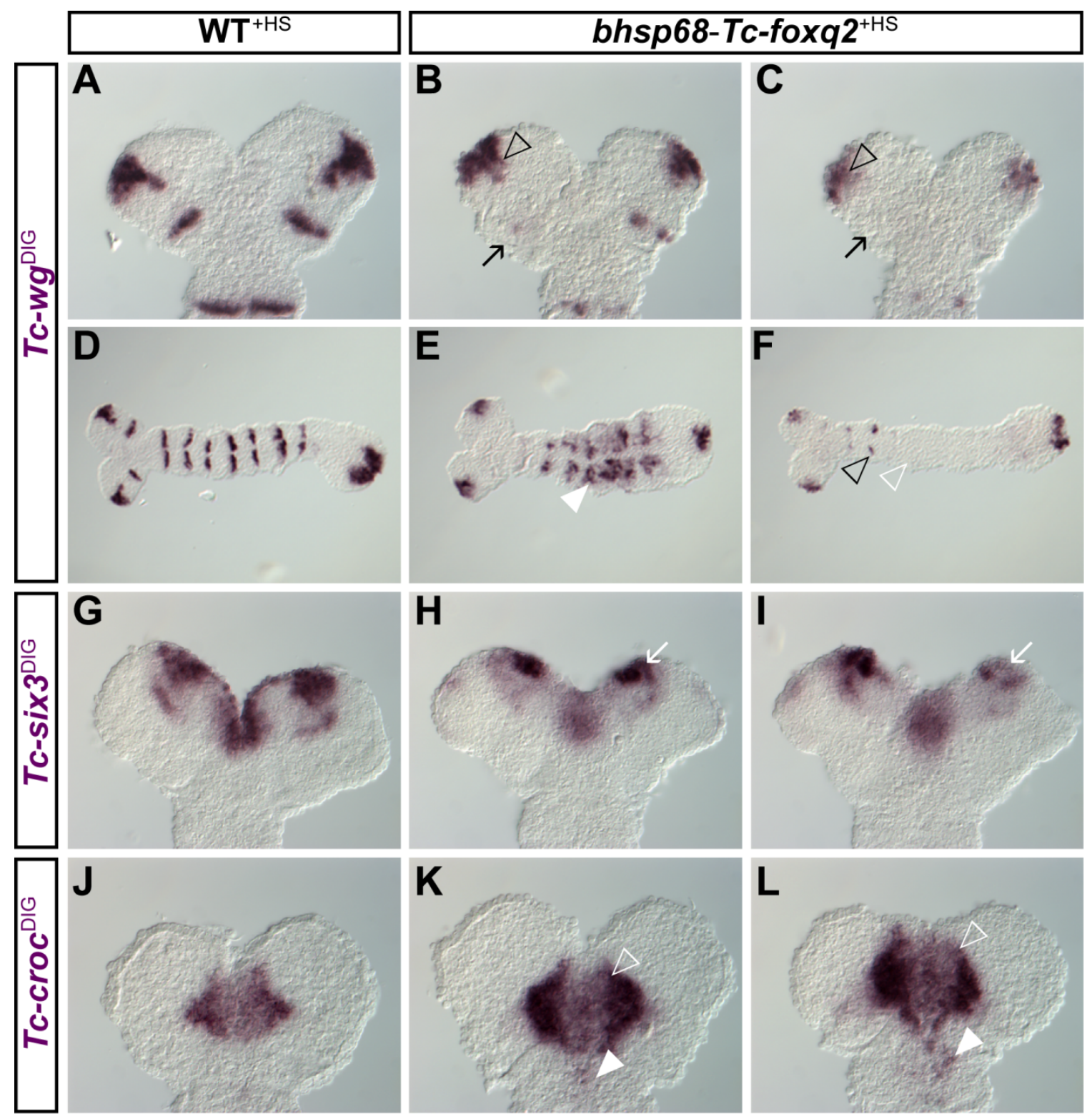

Figure 4.27 Ectopic Tc-foxq2 expression impacts head patterning gene expression profiles (mild effects).

Anterior is up (left in D-F). Expression of head marker genes in heat shock-treated wt (A, D, G, J) and hsp68-Tc-foxq2 (B, C, E, F, H, I, K, L) embryos (14-18 h AEL) is monitored by ISH. (B, C) The ocular Tc-wg expression domain is slightly (B) or heavily (C) reduced (empty arrowheads). The antennal expression domains are heavily reduced (B: arrow) or completely absent (C: arrow). (E, F) The Tc-wg stripes posterior to the procephalon are collapsed (E: white arrowhead), reduced (F: black empty arrow) or completely absent (F: white arrowhead), in hsp68-Tc-foxq2 ${ }^{+\mathrm{HS}}$ embryos. $(\mathbf{H}, \mathbf{I})$ The $T c-s i x 3$ expression domains within the neurogenic region are reduced (arrows). (K, L) The Tc-croc expression pattern appears to be slightly expanded (empty arrowheads) and posteriorly spread (arrowheads). 


\subsubsection{Tc-foxq2 is required for proper brain formation}

\subsubsection{Embryonic Tc-foxq2 knock-down causes neural phenotypes in L1 larvae}

Tc-six3 is known to be required for the proper formation of the brain (Posnien et al., 2011b). Embryonic Tc-six3 knock-down experiments resulted in defects of the central body, convergence of the brain hemispheres and reduction of the mushroom bodies in L1 larval stages (Posnien et al., 2011b).

Further, it has been shown for Tc-chx, a neuroendocrine marker of the pars intercerebralis (Posnien et al., 2011b), to be essential for the proper formation of the central body (Koniszewski, 2011). The findings that $T c$-foxq2 is co-expressed and interacting with $T c-s i x 3$ and $T c-c h x$ in neurogenic regions during embryogenesis raised the question whether $T c-f o x q 2$ has a function in neural development as well. In order to proof this hypothesis, I performed Tc-foxq2 pRNAi within the brainy reporter line and analyzed L1 larvae regarding neural defects, via confocal in vivo imaging (Posnien et al., 2011b). The brainy line is a reporter line that visualizes the neuropils and a subset of glial tissue (Koniszewski et al., 2016; Posnien et al., 2011b).

The knock-down of embryonic Tc-foxq2 function resulted in L1 larvae that showed different levels of neural defects $(n=13)$. The weakest phenotypes showed mushroom bodies with shorter medial lobes (Figure 4.28B', magenta marker) that appeared to be fused $(61.5 \%$; Figure 4.28: compare A and B: empty arrows). Further, the central complex appeared to be shortened (61.5\%; Figure 4.28B', yellow marker) and the brain hemispheres appeared to be more closely together or even fused (61.5\%; Figure $4.28 A^{\prime}, B^{\prime}:$ empty arrowheads). In stronger phenotypes of $T c$-foxq2 knock-down larvae the central complex was reduced in size as well, and the mushroom bodies were not detectable (23.1\%; Figure $\left.4.28 \mathrm{C}^{\prime}\right)$. Also the brain hemispheres in this phenotype were fused at the midline (Figure $4.28 \mathrm{C}, \mathrm{C}^{\prime}$ ). The strongest phenotype appeared to have no central complex, mushroom body, and antennal lobe at all $(n=7.7 \%$; Figure 4.28D, D'). The entire brain hemispheres seem to be heavily reduced in this phenotype. However, this class of phenotypes was hard to interpret as secondary effects may blur the primary phenotype. Interestingly, the strength of neural defects correlated with the strength of the epidermal defects. Weak neural defects also appeared with a size-reduced labrum, whereas strong defects lacked the complete labrum. Taken together, these data suggest that Tc-foxq2 has indeed a neural function, presumably required for correct formation of the central body and the mushroom bodies. Further, the observed neural defects caused by knock-down of Tc-foxq2 
function and the neural defects reported for Tc-six3 knock-down larvae resemble each other (Posnien et al., 2011b).
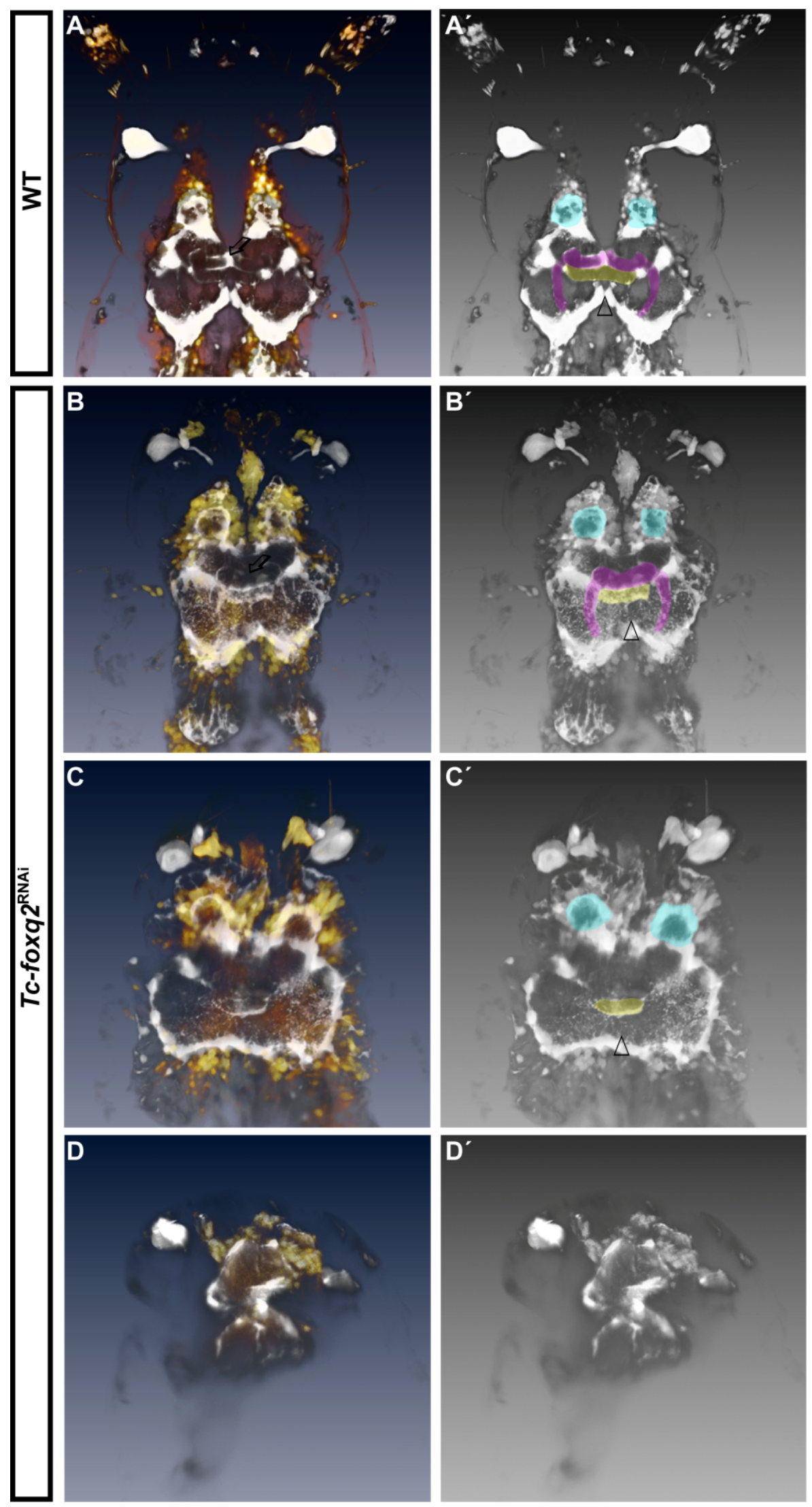
Figure 4.28 Embryonic knock-down of Tc-foxq2 function leads to defects in L1 larval brains. L1 larval brains are shown with anterior up, visualized with the transgenic brainy reporter line. In A (wt) and B-D $\left(T c-f o x q 2^{\text {pRNAi }}\right)$ neural cells are shown in yellow and glial cells in white. In $\mathbf{A}^{`} \mathbf{D}^{`}$ glial cells are shown in white and neuropils are color-coded. (A, $\mathbf{A}^{*}$ ) Wt L1 larval brain with two brain hemispheres, each with a mushroom body (magenta), an antennal lobe (cyan), and the mid-line spanning central body (yellow). (B, B') A weak Tc-foxq $2^{\text {pRNAi }}$ larval brain phenotype showing the loss of the boundary between the medial lobes of the mushroom bodies (compare arrows in $\mathbf{B}$ and $\mathbf{A}$ ). The central complex appears to be slightly reduced in size. $\left(\mathbf{C}, \mathbf{C}^{\prime}\right)$ Intermediate $T c-f o x q 2^{\text {pRNAi }}$ larval brains appear to lack the complete mushroom bodies. The central body is reduced in size. (D, D') Strong $T c-f o x q 2^{\text {pRNAi }}$ neural phenotypes show a completely disarranged and strongly reduced brain in L1 larvae. Furthermore the brain hemispheres appear to be more closely together or fused ( $\mathbf{A}^{\prime}-\mathbf{C}^{\prime}$ : empty arrowheads).

The Tc-foxq2 knock-down experiment in the brainy reporter line showed that the most pronounced neural defects were found in the mushroom bodies. To get a better view on these defects, I performed Tc-foxq2 pRNAi within the mushroom body reporter line ('MB-green'), which visualizes the overall structure of the mushroom body (Binzer et al., 2014; Koniszewski et al., 2016; Posnien et al., 2011b).

The experiment with the MB-green reporter line revealed different grades of phenotype strength. Only phenotypes that were observed at least twice are discussed. Knock-down of embryonic Tc-foxq2 most frequently led to L1 larvae that lacked the border between the two medial lobes, indicating a fusion of the medial lobes (Figure 4.29B: empty arrowhead, compare with Figure 4.28B). Moreover, L1 larvae were frequently observed, which appeared to have intact but misarranged mushroom bodies, resulting in a loss of contact between the medial lobes from the two hemispheres (Figure 4.29C: arrowheads). In intermediate phenotypes, the mushroom bodies were twisted with each other at the midline (Figure 4.29D). In strong phenotypes appeared the mushroom bodies to be completely absent (Figure 4.29E). Intermediate and strong phenotypes were scarcely found. These findings substantiate the result observed within the brainy reporter line.

Taken together, the experiments using the neural reporter lines indicate that $T c-f o x q 2$ is required for the correct formation and arrangement of the mushroom bodies, for the proper formation of the central body and led upon knock-down to fused brain hemispheres. Interestingly, the neural defects observed in Tc-foxq2 larvae are similar to the defects in $T c$-six 3 knock-down larvae (Posnien et al., 2011b). 


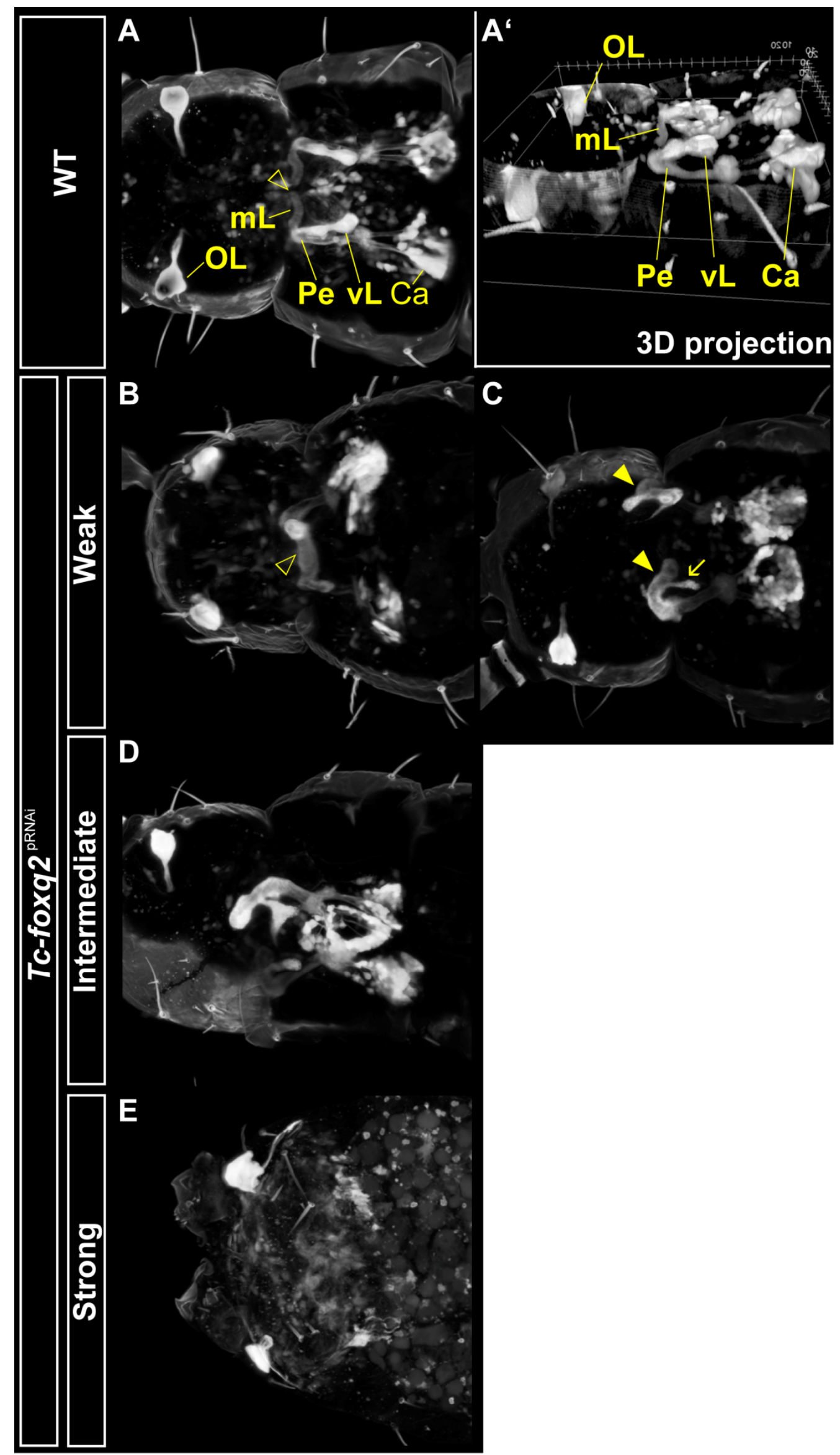


Figure 4.29 Embryonic Tc-foxq2 knock-down leads to mushroom body defects in L1 larvae. Anterior is left. (A-F) Mushroom bodies are in L1 larvae visualized by using the transgenic MB-green reporter line. (A) Maximum projection of wt L1 larval mushroom bodies in a dorsal view. (A') 3D projection of wt L1 larval mushroom body in a lateral view, providing a better overview of the organization of the structures. (B) A weak Tc-foxq2 mushroom body phenotype, which lacks the border at the midline between the two medial lobes (compare empty arrowheads in A and B). (C) Tc-foxq2 ${ }^{\text {pRNAi }}$ L1 larval mushroom bodies, which show distorted pedunculi, leading to a loss of contact between the two medial lobes (arrowheads), and slightly reduced vertical lobes (arrow). (D) Intermediate $T c-f o x q 2^{\mathrm{pRNAi}}$ mushroom body phenotypes are either marked by an interdigitation of the two mushroom bodies (D). (E) In strong Tc-foxq $2^{\text {pRNAi }}$ larval brain phenotypes the mushroom body structures are highly reduced or absent. OL: optical lobe, mL: medial lobe, Pe: pedunculus, vL: vertical lobe, Ca: calyx

\subsubsection{Embryonic Tc-foxq2 gain-of-function results in larvae showing neural}

\section{phenotypes}

I was also interested whether Tc-foxq2 gain-of-function embryos result in larvae with neural defects. Thus, I crossed the ectopic Tc-foxq2 expression line hsp68-Tc-foxq2 with the brainy as well as with the MB-green line. Embryonic offspring (0-24 h AEL) of these hybrids was then heat shocked and the emerged L1 larvae were analyzed regarding neural deficiencies.

Heat shock treatment of both the brainy and the MB-green reporter lines alone did not lead to an increase of specific neural defects in larvae (data not shown). Heat shock-treated double heterozygous animals (reporter lines crossed with the $h s p 68-T c$-foxq2 line) showed mild neural defects. The hemispheres looked normal (Figure 4.30A-D'), but the medial lobes of the mushroom bodies appeared to be slightly elongated and the pedunculi appeared to be slightly misarranged (Figure 4.30B', magenta marker). In some cases the central body was elongated or its shape slightly altered (Figure 4.30C', D', yellow marker) and sometimes the medial lobes lost contact (Figure 4.30C', magenta marker). The observed mushroom body phenotype was confirmed in the MB-green line. The mushroom bodies of these larvae appeared to have all units. However, the medial lobes of the mushroom bodies had lost contact to each other (Figure 4.31, compare B with A: yellow brackets) or were folded posteriorly (Figure 4.31C, empty arrowheads). In some cases he pedunculi appeared slightly dislocated (Figure 4.31D, arrowheads).

Taken together, the neural defects of the $T c$-foxq 2 gain-of-function assay were less severe than the defects in the Tc-foxq2 knock-down assay. However, both assays showed affected 
mushroom bodies and central bodies, while the antennal lobes were not affected. Both assays showed dislocated medial lobes, which had lost contact, while only in the knock-down assay the mushroom bodies appeared to be fused. Further, loss of the central body and mushroom bodies were only found in the knock-down assay, while the gain-of-function assay showed enlarged medial lobes. Moreover, the midline fusion of the two brain hemispheres was only observed in the knock-down assay. 

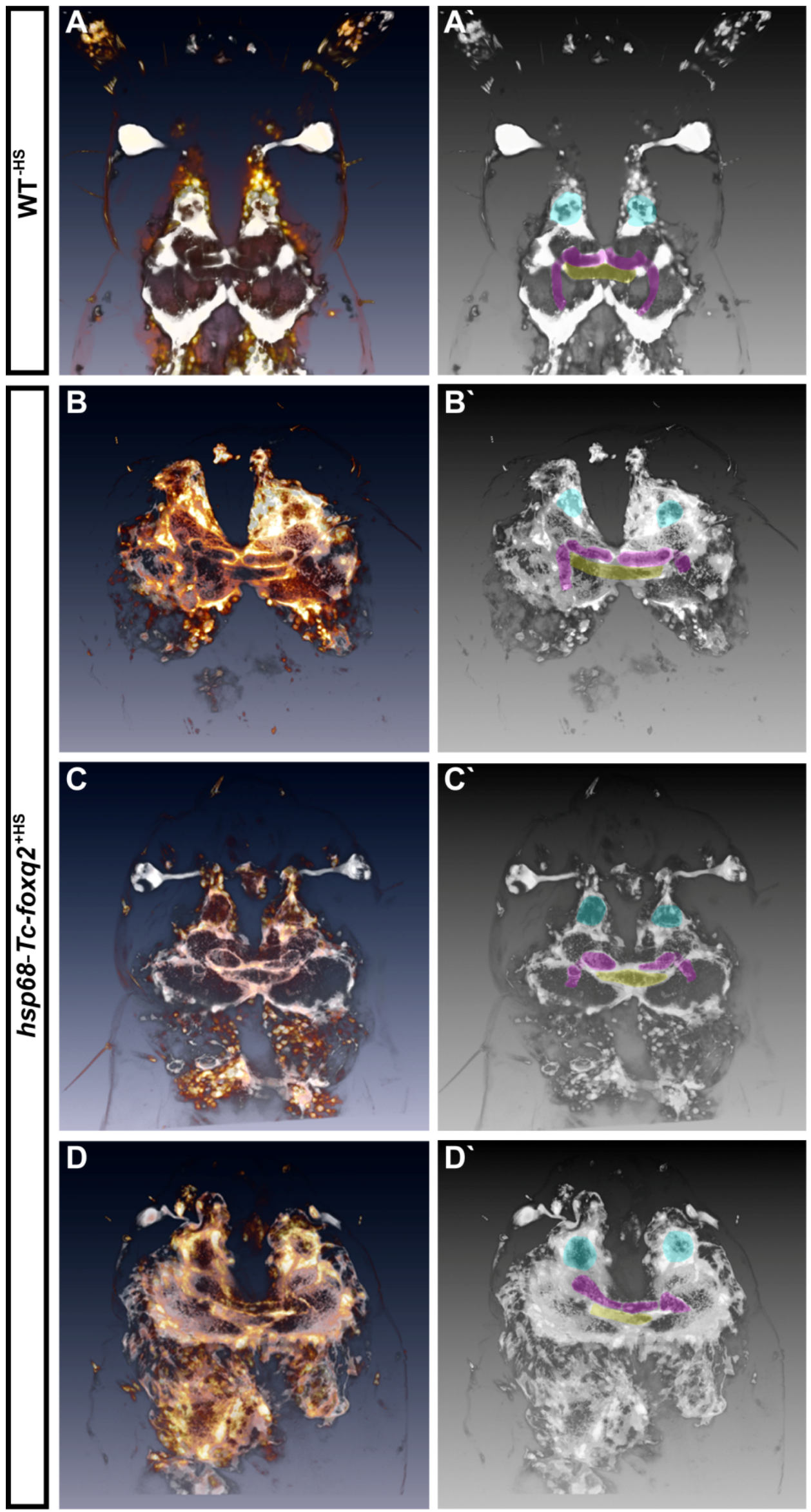
Figure 4.30 Embryonic gain of Tc-foxq2 function leads to weak neural defects in L1 larvae. L1 larval brains are shown with anterior up, visualized using the transgenic brainy reporter line. In A (wt) and B-D (hsp68-Tc-foxq2 ${ }^{+\mathrm{HS}}$ ) neural cells are shown in yellow and glial cells in white. In $\mathbf{A}^{`}$ - $\mathbf{D}^{`}$ glial cells are shown in white and neuropils are color-coded. (A, $\left.\mathbf{A}^{\prime}\right)$ Wt L1 larval brain with two brain hemispheres, each with a mushroom body (magenta), an antennal lobe (cyan), and the mid-line spanning central body (yellow). (B, B') L1 larval brain that shows enlarged medial lobes (magenta) and a slightly misarranged pedunculus after ectopic Tc-foxq2 expression (magenta). (C, C') L1 larval brain showing dislocated medial lobes (magenta) and a shape-altered central body (yellow). (D, D') L1 larval brain with a slightly reduced central body (yellow) and dislocated pedunculi (magenta). 


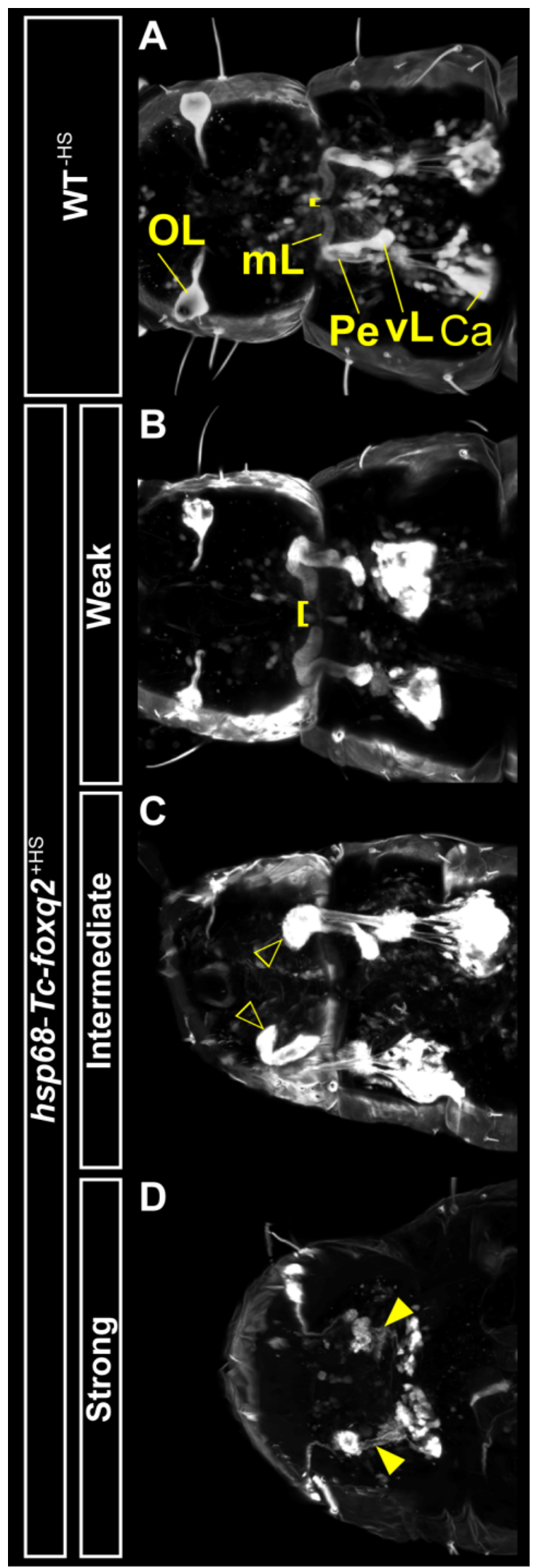

Figure 4.31 Embryonic gain of Tc-foxq2 function leads to affected mushroom bodies in L1 larvae. Anterior is left. (A-F) L1 larval mushroom bodies are visualized by using the transgenic MB-green line. (A) Maximum projection of wt L1 larval mushroom bodies in a dorsal view. (B) Weak phenotypes are marked 
by an enlarged gap between the medial lobes of the two hemispheres (compare brackets in $\mathbf{A}$ and $\mathbf{B}$ ). (C) Intermediate phenotypes show strongly dislocated medial lobes (empty arrowheads). (D) The strongest $h s p 68-T c-f o x q 2^{\text {HS }}$ phenotypes show reduced and misarranged mushroom bodies, in which the medial lobes appear to be absent. OL: optical lobe, $m L$ : medial lobe, Pe: pedunculus, vL: vertical lobe, Ca: calyx

\subsubsection{Tc-foxq2 misregulation leads to increased cell death rates within the}

\section{neurogenic head region}

Trying to track down possible reasons for the neural phenotype in Tc-foxq 2 knock-down experiments, I analyzed the cell death rate in this particular region for several embryonic stages ( $<30 \mathrm{~h} \mathrm{AEL}$ ). To this end, I performed virtually the same experiment as in 4.1.2.2, with the sole difference that the ROI was not the labral but the neurogenic head region (Figure 4.32 $A_{a}$, region 2).

The first embryonic stages (germ rudiment to fully elongated stages) showed no differences in cell death rates in $T c$-foxq $2^{\text {pRNAi }}$ embryos compared to wt embryos (Figure 4.32B: stage 1-4). However, retracting germ bands showed a significant increase in the cell death rate within the neurogenic head region (Figure 4.32B: stage $5 ; p=0.023$ ). Within the neurogenic region, the vast majority of apoptotic cells were located at the anterior rim of the head lobes (Figure 4.32 $\mathrm{A}_{b}$, empty arrowhead). This region is important for neural development. Tc-six3 and Tc-chx are markers of the pars intercerebralis, both are expressed at this region, and lead to neural defects resembling the $T c$-foxq2 neural defects, upon knock-down (Posnien 2011). Further, it is known that neuroblasts, which are involved in central complex formation, form within this region in retracting embryos (Boyan and Reichert, 2011; Koniszewski, 2011). This indicates that increased cell death rates could be related to the observed $T c$-foxq 2 knock-down neural phenotype. 


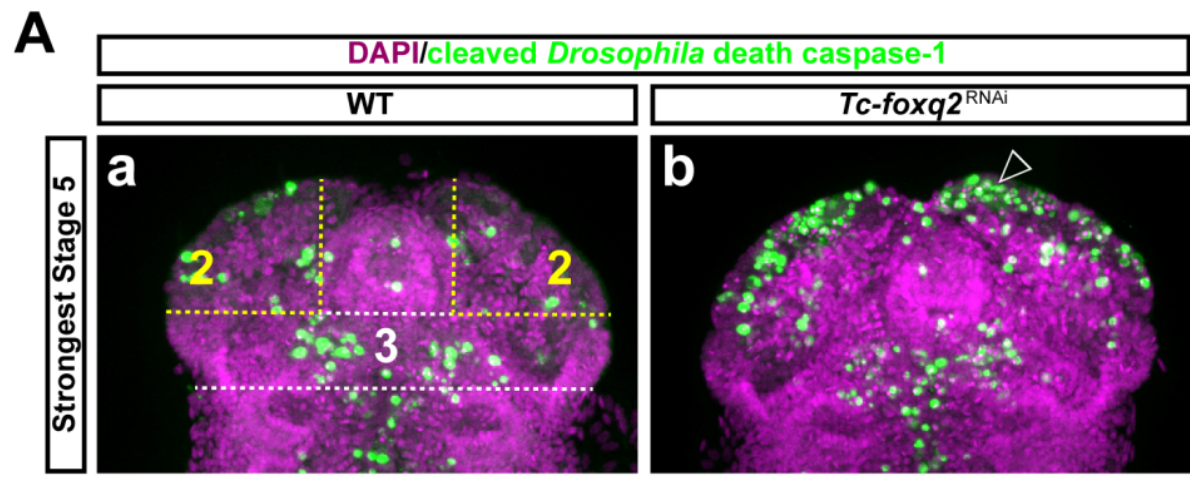

B

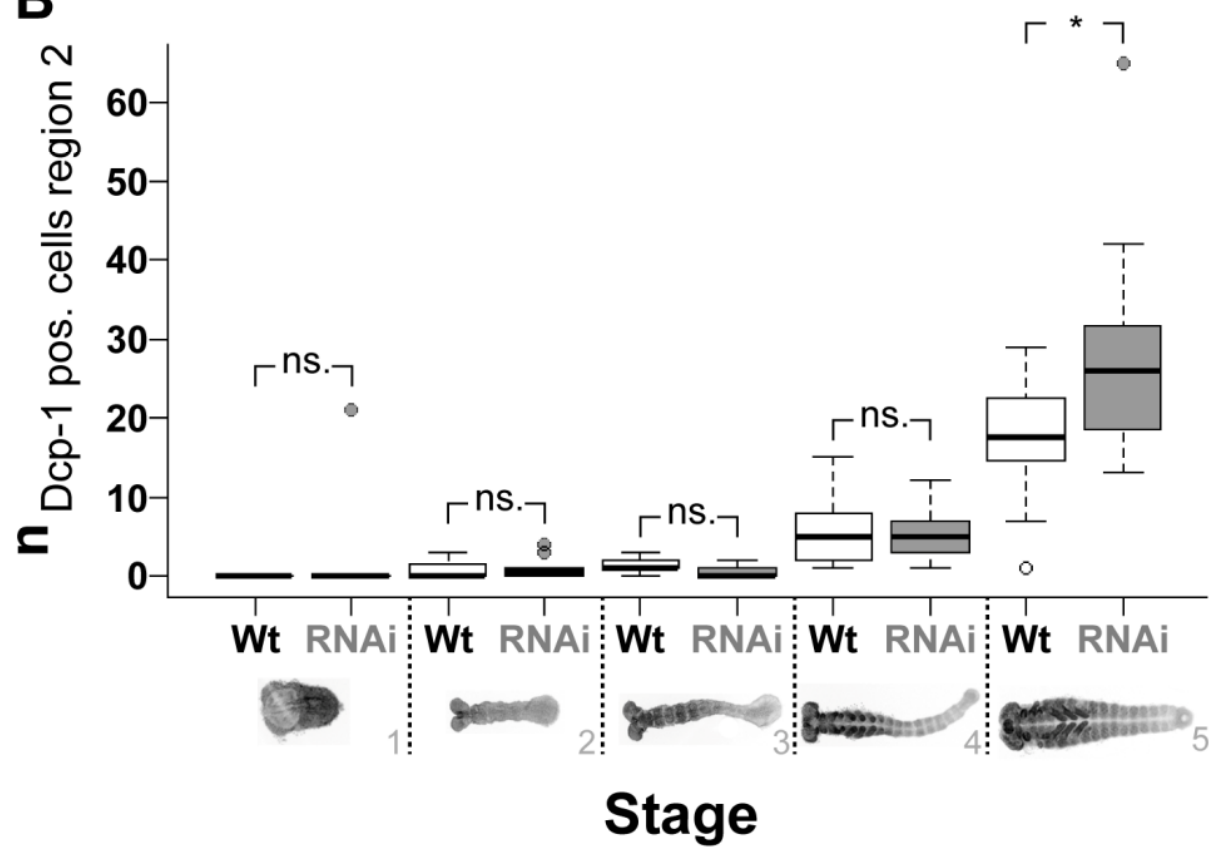

Figure 4.32 Analysis of cell death rates within the neurogenic head region in $T c$-foxq2 $2^{\text {pRNAi }}$ embryos. Anterior is up $\left(\mathbf{A}_{a}, \mathbf{A}_{b}\right)$. Apoptotic cells, in wt $\left(\mathbf{A}_{a}\right)$ and in $T c$-foxq2 ${ }^{\text {pRNAi }}\left(\mathbf{A}_{b}\right)$ embryos, are monitored by antibody staining (Dcp-1 - Alexa Fluor 488, green). Nuclei are stained (DAPI, magenta) to visualize embryonic morphology. $\left(\mathbf{A}_{a}, \mathbf{A}_{b}\right)$ Retracting germ bands with the highest number of apoptotic cells in wt $\left(\mathbf{A}_{\mathbf{a}}\right)$ and $T c$-foxq $2^{\text {pRNAi }}\left(\mathbf{A}_{\mathbf{b}}\right)$ embryos. Indicated are the neurogenic region (ROI 2, yellow dashed lines) and the region, which was used for normalization of the data set (region 3, white dashed lines). The $T c-f o x q 2^{\text {pRNAi }}$ retracting germ band shows a strong accumulation of apoptotic cells within the neurogenic region $\left(\mathbf{A}_{b}\right)$. (B) Box plot depicting the number of apoptotic cells ( $y$-axis) versus five different embryonic stages. The ROI 2 values are normalized with the region 3 values. Brackets display grade of significance. Germ rudiments (stage 1) to fully elongated germ bands (stage 4) show no significant increase of apoptotic cells (stage 1: $p=0.33$ (wt: $n=3$, RNAi: $n=7$ ), stage 2: $p=0.35$ (wt: $n=11$, RNAi: $n=12$ ), stage 3: $p=0.99$ (wt: $\mathrm{n}=9$, RNAi: $\mathrm{n}=19$ ), stage 4: $p=0.23$ (wt: $\mathrm{n}=17$, RNAi: $\mathrm{n}=15)$ ). However, retracting germ bands showed in the ROI 2 significantly more apoptotic cells ( $p=0.023)$ in RNAi embryos $(n=11)$ compared to untreated embryos ( $n=12)$. ns.: not significant 


\subsection{Expanding the Tribolium toolbox}

\subsubsection{Generating transgenic lines driving strong and ubiquitous expression of a nuclear localized EGFP}

Nuclear reporter lines are well-suited for in vivo imaging experiments (Clarkson and Saint, 1999; El-Sherif et al., 2012; Pauls et al., 2001; Sarrazin et al., 2012; Strobl et al., 2015). However, for embryonic in vivo imaging experiments the signal of the reporter line needs to (1) be strong enough for imaging, (2) mark all nuclei, and (3) be always localized to the nucleus. The first reported transgenic Tribolium nuclear reporter line used for in vivo imaging was the EFA-nGFP line (El-Sherif et al., 2012; Sarrazin et al., 2012). This line encodes the green fluorescent protein (GFP) with a nuclear localization signal (nls or n; nGFP) under the control of the Tc-elongation factor $1 \alpha$ (TC-EFA) promoter, which should drive ubiquitous expression. This line was well-suited for some questions, but it has also two major drawbacks. First, the nGFP signal gets blurry during the Prophase of mitosis (Figure 4.33F: box), because of the breakdown of the nuclear envelope (Smoyer and Jaspersen, 2014) and the efflux of the nGFP into the cytoplasm. Furthermore, the Tc-EFA promoter does not drive complete ubiquitous expression, showing an uneven signal distribution throughout the embryo (Figure 4.330: box). On the basis of these findings, I tried to generate transgenic lines, which effectively drive ubiquitous and strong expression of a fluorescent protein, which is DNA-bound, and does therefore not show a blurry signal during mitotic stages. To this end, I generated a chimeric protein, which consists of the histone Tc-H2A variant (Tc-H2Av) and the fluorescent protein enhanced GFP (EGFP; H2Av::EFGP). Afterwards I cloned three different constructs each with a different promoter ( $T c$-alpha tubulin 1 promoter (aTub1P; Siebert et al., 2008), Tc-polyubiquitin promoter (PUbP) and Tc-ribosomal protein subunit 3 promoter (rps3P)), which should drive strong and ubiquitous expression of the chimeric H2Av::EGFP protein. I cloned these reporter cassettes into a transgenesis vector and generated three different imaging lines. Subsequently I analyzed them concerning their expression profile and compared them to the EFA-nGFP imaging line.

\subsubsection{Qualitative promoter comparisons}

I compared the three generated imaging lines (rps3P-H2Av::EGFP, $\alpha$ Tub1P-H2Av::EGFP, and PUbP-H2Av::EGFP) with each other and with the already published EFA-nGFP line. To this end, I 
imaged all lines with the same microscope settings and processed them all in same the way. Afterwards, I analyzed these lines regarding the signal localization, intensity, and distribution as well as with regard to their viability. However, the PUbP-H2Av::EGFP imaging line died after some weeks. Thus, the detailed description is restricted to the lines, which survived. The new lines were a pool of hetero- and homozygous animals, while the EFA-nGFP line was completely homozygous. For the comparison only individuals of each line with the strongest signal intensity were used.

At first I compared the different lines regarding the promoter-based activation intensity and the signal localization at embryonic stages (Figure 4.33A-O). The comparison revealed that the $\alpha T u b 1 \mathrm{P}-H 2 A v:: E G F P$ line showed the strongest signal intensity at blastoderm stages (Figure 4.33B). However, in the course of development the signal intensity of the $\alpha$ Tub1P-H2Av::EGFP line got weaker (Figure 4.33B, E, H, K, N) and showed signal intensities comparable to the signal of the rps3P-H2Av::EGFP line (Figure 4.33M). In contrast, the signal intensity of the EFA-nGFP line became stronger during these stages (Figure 4.33C, F, I, L, O). As a consequence, retracting germ band stages showed the strongest signal intensity in the EFA-nGFP line (Figure 4.330). The aTub1P-H2Av::EGFP and the rps3P-H2Av::EGFP line showed an ubiquitous signal with no obvious expression gaps. In contrast, the EFA-nGFP line showed regions where the signal was not detectable (Figure 4.33 compare M: box with O: box). The signal of the fluorescent chimeric protein of both of the new lines was always sharp and tightly localized to the DNA (Figure 4.33D: box), whereas the signal of the EFA-nGFP line was blurry and distributed over the whole cell during mitosis (Figure 4.33F: box). The blurry, equally distributed signal is due to the breakdown of the nuclear envelope in the Prophase (Smoyer and Jaspersen, 2014), leading to an efflux of the unbound nuclear localized nGFP.

I also compared signal intensities of the different lines at larval (Figure 4.34A-C), pupal (Figure 4.34D-F), and adult stages (data not shown). At larval as well as at pupal stages the $\alpha T u b 1 \mathrm{P}-H 2 A v:: E G F P$ line showed the strongest signal intensities (Figure 4.34B, E). Signal intensities of the rps3P-H2Av::EGFP (Figure 4.34A, D), and the EFA-nGFP (Figure 4.34A, D and C, F) line had comparable signal intensities. In adult stages the $\alpha$ Tub1P-H2Av::EGFP and the EFA-nGFP line showed the best signal intensities (data not shown).

Comparison of the distribution, localization, and intensities of the signal showed no considerable differences in ovaries among the different lines. In all imaging lines a signal was found within the follicle cells, the nurse cells as well as in the pro-nucleus in germ cells (Figure 4.35A-C'). 
However, in all lines the signal of the pro-nucleus was not always detectable (Figure 4.35B-C'). The basis of this phenomenon was not further analyzed.

Taken together, the PUbP-H2Av::EGFP line showed the strongest signal intensities at all developmental stages (Figure 4.36), but the line was not viable. Comparing the aTub1P-H2Av::EGFP line with the rpS3P-H2Av::EGFP line, the $\alpha$ Tub1P-H2Av::EGFP line showed comparable or even better signal intensities at the analyzed developmental stages (Figure 4.36). Both lines showed similar viabilities (Figure 4.36). In contrast, the EFA-nGFP line showed the best viability and the strongest signal intensities at post-elongation germ band stages, with the drawback of signal gaps in the embryonic tissue and blurry signals in dividing cells (Figure 4.36). It is possible that the strong expression of histone-tagged EGFP is interfering with viability (see section 5.2.3), which could restrict the maximal signal intensity. 


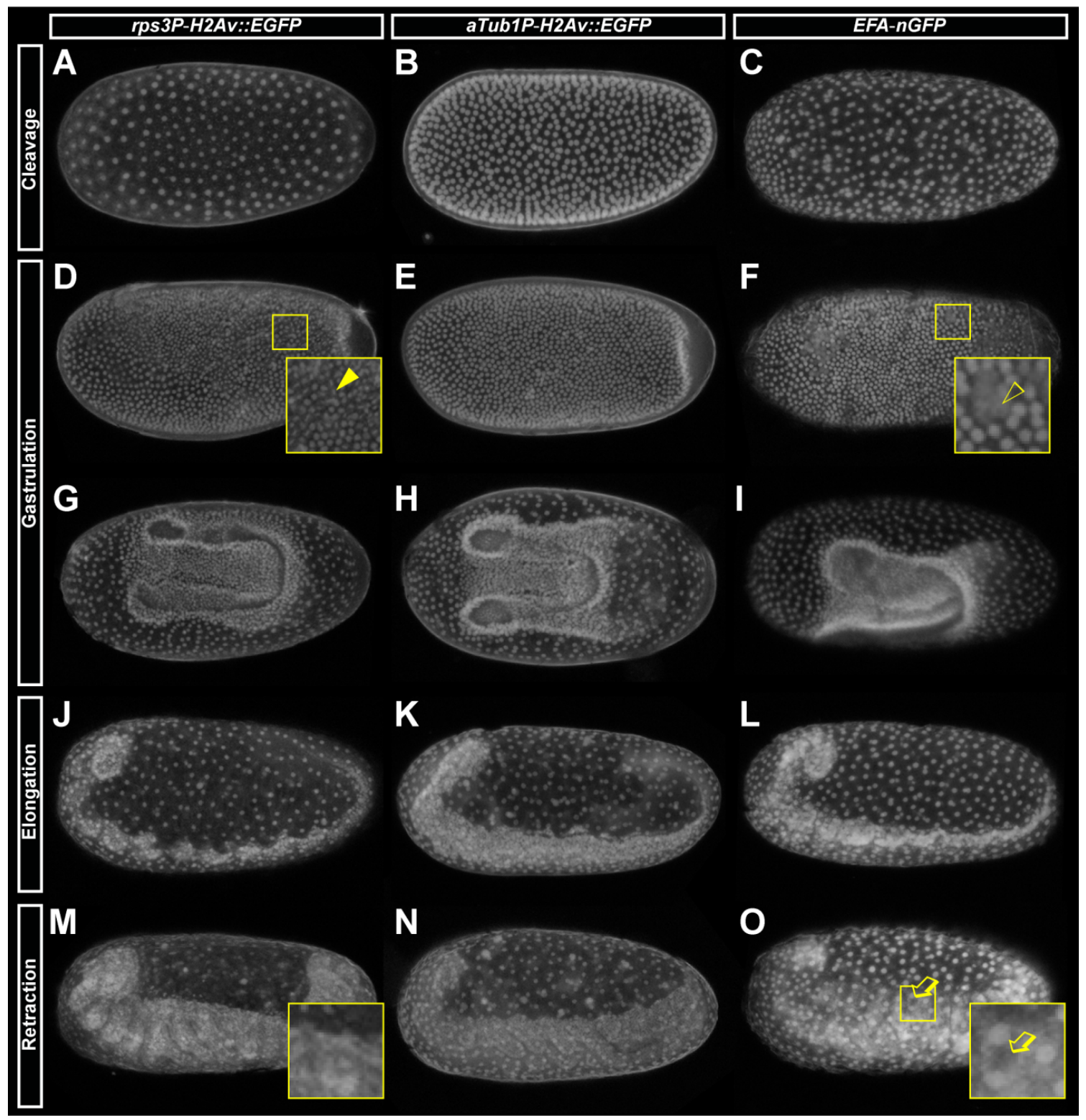

Figure 4.33 Qualitative comparison of signal intensities and localizations of three different ubiquitous nuclear reporter lines at early embryonic stages. Anterior is left. Nuclei are visualized by using the transgenic imaging lines rps3P-H2Av::EGFP (A, D, G, J, M), $\alpha \operatorname{Tub1P-H2Av::EGFP}(\mathbf{B}, \mathbf{E}, \mathbf{H}, \mathbf{K}, \mathbf{N})$, and EFA-nGFP (C, F, I, L, O, El-Sherif et al., 2012). The embryos are depicted as average projections. (A-C) Blastoderm stages of the $\alpha$ Tub1P-H2Av::EGFP line (B) show the strongest and blastoderm stages of the rps3P-H2Av::EGFP line (A) the weakest signal intensity. (D-F) With the onset of the amniotic fold all three lines show comparable signal intensities. (G-0) At later stages the signal of the EFA-nGFP line becomes successively stronger and shows the strongest signal intensity in all subsequent stages (I, L, $\mathbf{O})$. In contrast, the $\alpha$ Tub1P-H2Av::EGFP line shows a successive decrease of the signal intensity $(\mathbf{H}, \mathbf{K}, \mathbf{N})$, and consequently shows the weakest signal intensity in retracting germ bands $(\mathbf{N})$. However, the advantages of the two new nuclear reporter lines, rps3P-H2Av::EGFP and $\alpha$ Tub1P-H2Av::EGFP, are that they show a crisp and DNA-associated signal also during mitosis (compare D: box, yellow arrowhead with F: box, yellow 
empty arrowhead) and that the signal is ubiquitously detectable, without signal gaps in the embryonic tissue (compare $\mathbf{M}$ : box with $\mathbf{0}$ : box, empty arrow).

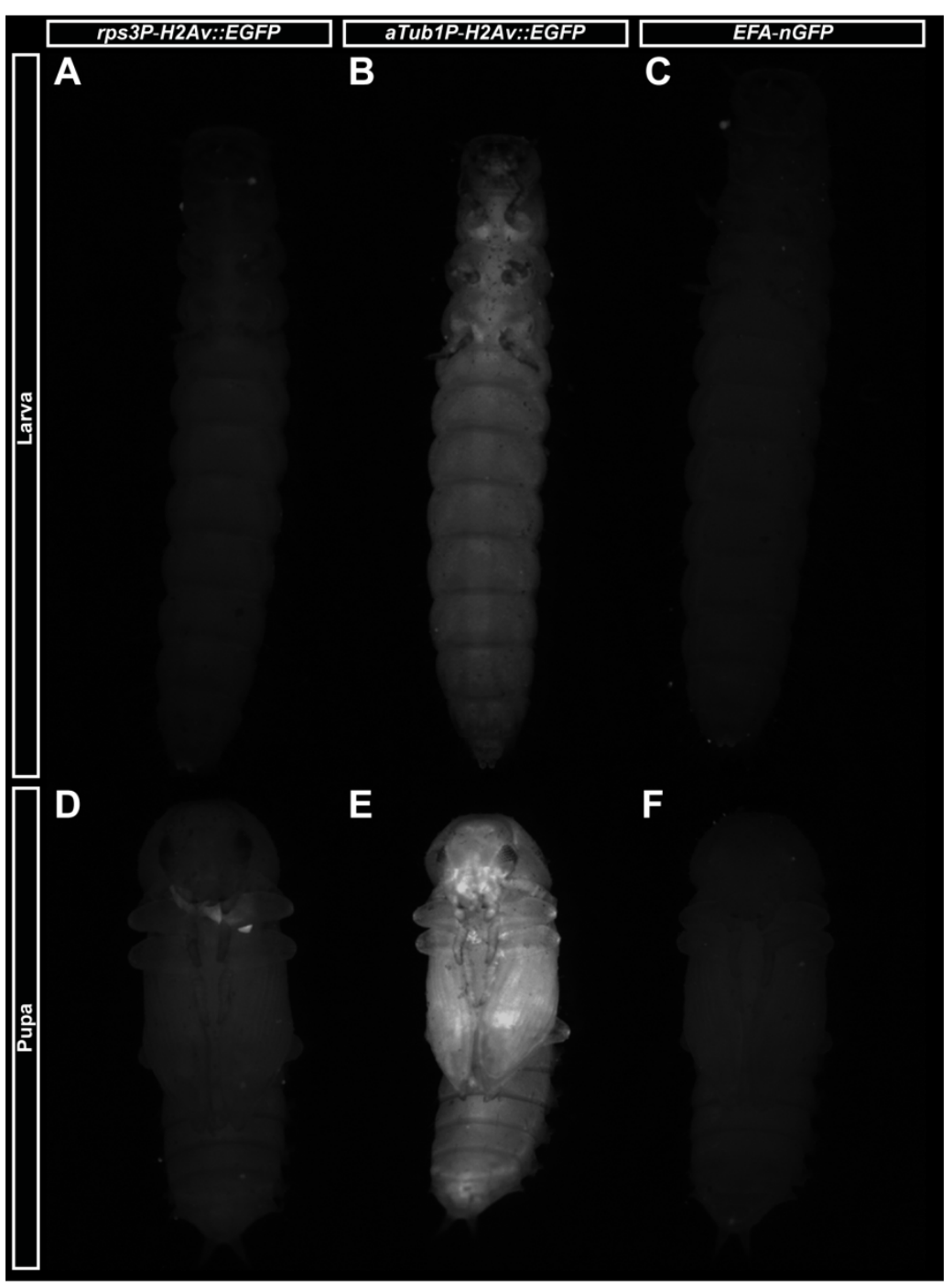

Figure 4.34 Qualitative comparisons of signal intensities of three different ubiquitous nuclear reporter lines at larval and pupal stages. Anterior is up. Larvae and pupae are monitored by using the transgenic imaging lines rps3P-H2Av::EGFP (A, D), $\alpha$ Tub1P-H2Av::EGFP (B, E) and EFA-nGFP (C, F, El-Sherif et al., 2012). (A-F) The $\alpha$ Tub1P-H2Av::EGFP line shows the strongest signal intensity at larval (B) as well as at pupal stages (E). The other lines show weak signals, which are comparable with respect to intensity. 


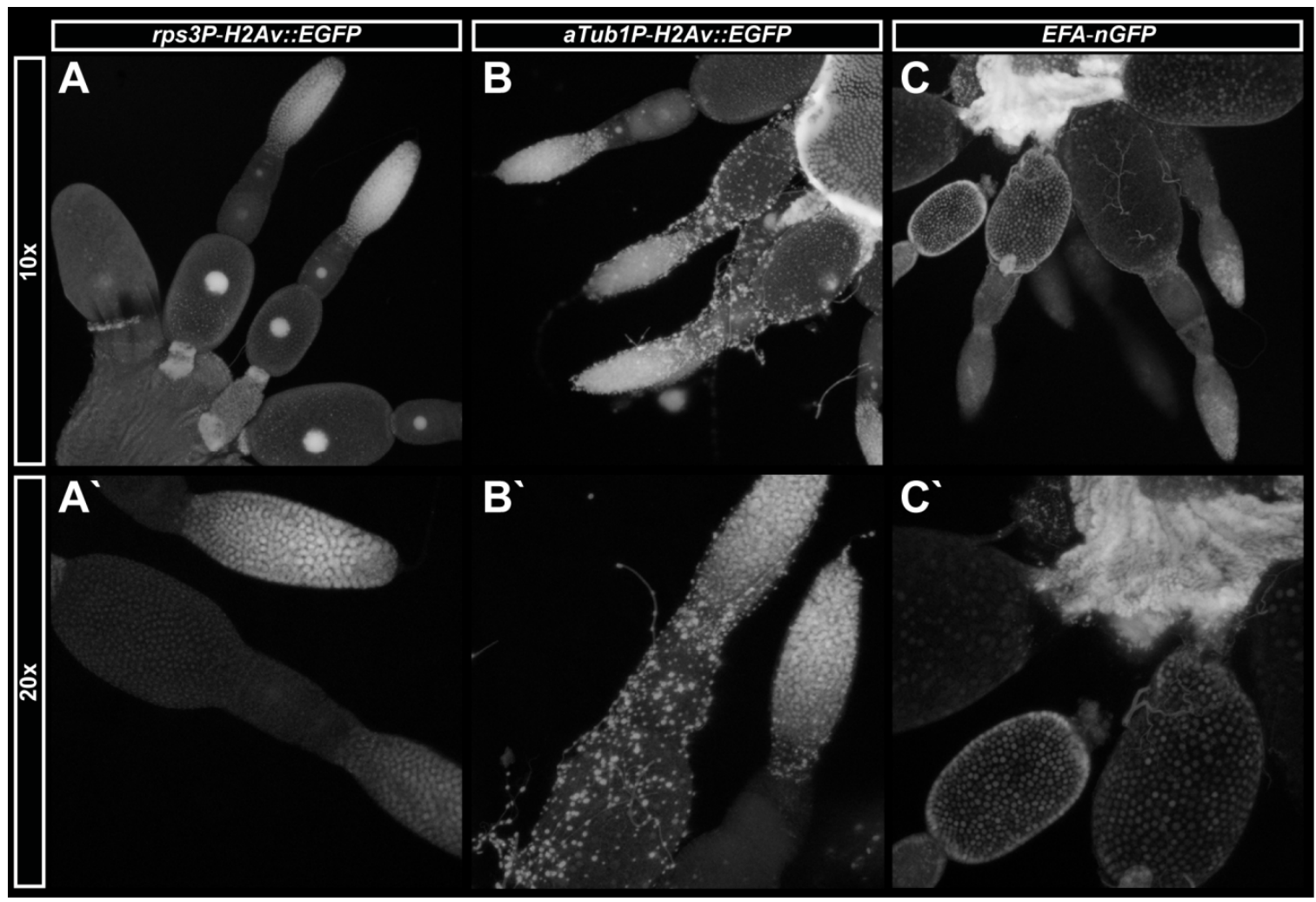

Figure 4.35 Qualitative comparison of signal intensity and localization of three different ubiquitous nuclear reporter lines in ovaries. Nuclei are visualized using the transgenic imaging lines rps3P-H2Av::EGFP (A, A'), $\alpha$ Tub1P-H2Av::EGFP (B, B') and EFA-nGFP (C, C'; El-Sherif et al., 2012). The dissected ovaries are depicted as maximum projections. (A-C') All three transgenic lines show comparable signal intensities in all structures of the ovaries. Although, only in the ovary of the rps3P-H2Av::EGFP line depicted $(\mathbf{A})$, could be in all three lines ovaries observed that show a signal in the pro-nucleus of germ cells.

\begin{tabular}{|c|c|c|c|c|}
\hline Promoter & Embryonic & $\begin{array}{l}\text { Signal } \\
\text { Larval/Pupal }\end{array}$ & Adult & Viability \\
\hline$\alpha T u b 1 P-H 2 A v:: E G F P$ & $\Rightarrow$ & $\pi$ & $\lambda$ & 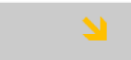 \\
\hline rps3P-H2Av::EGFP & 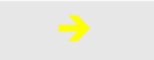 & 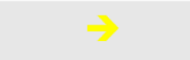 & 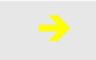 & $y$ \\
\hline PUbP-H2AV::EGFP & $\uparrow$ & 个 & 个 & $\downarrow$ \\
\hline$E F A-n G F P$ & 个* & $*$ & त्र* & $\uparrow$ \\
\hline
\end{tabular}

Figure 4.36 Summary of the qualitative analysis of four different ubiquitous nuclear reporter lines. The strongest signal was detectable in the transgenic PUbP-H2Av::EGFP nuclear reporter line. However, due to low viability the line died out before a detailed analysis could be started. The $\alpha$ Tubulin1P-H2Av::EGFP line showed the best quality, due to stronger signals especially at post-embryonic stages. The already published EFA-nGFP line (El-Sherif et al., 2012) was the best, considering the signal at early embryonic stages and the viability. However, a patchy expression pattern and the blurry signal during mitosis are drawbacks (asterisks). 


\subsubsection{Ubiquitous H2Av::EGFP expression - Proof of principle}

In order to test whether the new imaging lines are suitable for future in vivo imaging experiments, I performed a first proof of principle experiment using the aTub1P-H2Av::EGFP imaging line. Collaborating with Dr. Sven Poppelreuther (Carl Zeiss Microscopy GmbH), I had the opportunity to image the line with the Lightsheet Z.1 microscope (ZEISS) for several hours (18 $\mathrm{h}$, 3-21 h AEL). We imaged simultaneously from a dorsal and ventral view in time intervals of three minutes. The video (see section S7.29 and the representative time frames: Figure 4.37) shows that the $\alpha$ Tub1P-H2Av::EGFP imaging line is functional, showing a signal, which is localized to the DNA and is equally distributed over the embryo throughout the stages (Figure 4.37A-J). Each nucleus appears to have a strong signal, which reveals the overall morphology of the embryo and key steps of early embryonic development like gastrulation (Figure 4.37B), the amniotic fold (Figure 4.37C), germ band elongation (Figure 4.37D-J) and limb bud growth (Figure 4.37J). Based on this result, I also collaborated with Dr. Stefan Münster (Tomancak Lab; MPI-MCBG in Dresden), trying to further exhaust imaging using the Lightsheet Z.1 microscope, and specifically in order to record videos, which are well suited for cell tracking and fate mapping. To this end, we tried to record several in vivo imaging videos of developing embryos. However, most of them died during the process only one embryo survived the treatment. The video was taken from three different views (with $180^{\circ}$ spacing each) and a time interval of three minutes. Afterwards, the video was completely processed by Dr. Stefan Münster, including registration and rendering (see section S7.30 and representative time frames: Figure 4.38). The video depicts a rendered $3 \mathrm{D}$ projection of an embryo (1.5-24 $\mathrm{h} \mathrm{AEL}$ ) from a lateral view. The video shows that light-sheetbased fluorescence microscopy (LSFM) is in combination with the $\alpha$ Tub1P-H2Av::EGFP imaging line a powerful tool, but the recorded video is so far not well suited for fate mapping and especially not for automatic cell tracking, due to a bad signal to noise ratio, resolution and the signal of the extra-embryonic tissue, which partially covers the embryonic signal.

More interested in the morphogenetic movements of the head, I also tried to image only the head in order to perform cell tracking and fate mapping experiments. To this end, I imaged the anterior cap using the $\alpha$ Tub1P-H2Av::EGFP line with the LSM 780 microscope (ZEISS) and Dr. Stefan Münster did the same with the Lightsheet Z.1 microscope. These two videos (see section S7.31 and S7.32, representative frames: Figure 4.39) give a short impression about the morphogenetic movement of the head. Both microscopes are suitable for this purpose in principle, but the LSFM technique allows following up complete morphogenesis of the head with its potential to image from different angles. With head development starting at a mid-ventral region of the egg (Figure 4.37C) and ending dorsally at the anterior pole (Figure 4.37J), it is only 
possible to record certain time frames of the development by using the LSM. Thus, the LSFM technique is more suitable for long-term cell tracking of the embryonic head development, whereas conventional confocal imaging could be used to image certain aspects of development.

Taken together, these proof of principle experiments indicate that the $\alpha$ Tub1P-H2Av::EGFP imaging line is suitable for in vivo imaging experiments as well as in combination with LSFM.

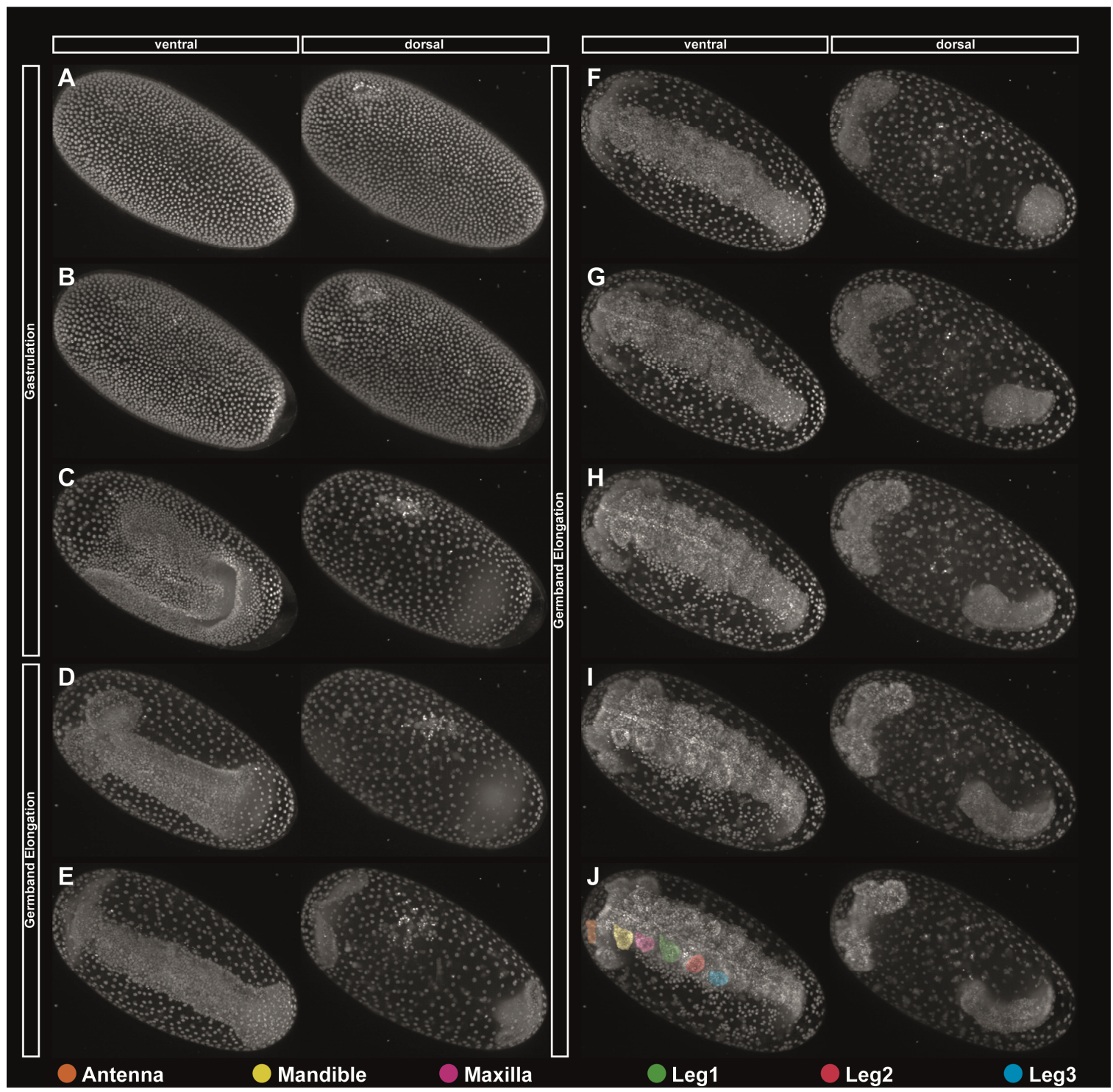

Figure 4.37 Early embryonic development imaged using the transgenic $\alpha$ Tubulin1P-H2Av::EGFP line in combination with LSFM technique I. Anterior is left. Nuclei are visualized by using the transgenic $\alpha$ Tub1PH2Av::EGFP nuclear reporter line. Ventral and dorsal views were simultaneously imaged and are depicted as maximum projections. (A) Blastoderm stage, which has finished the last round of cell division. (B) Start of amniotic fold. (C) Formation of the germ rudiment. (D-J) Germ band elongation. Note that the anterior head is first localized at a ventral sub-terminal position within the egg (C) and ends up at the anterior cap of the egg $(\mathrm{J})$. Limb buds start to grow out $(\mathbf{G})$ and became later clearly recognizable $(\mathrm{J})$. 


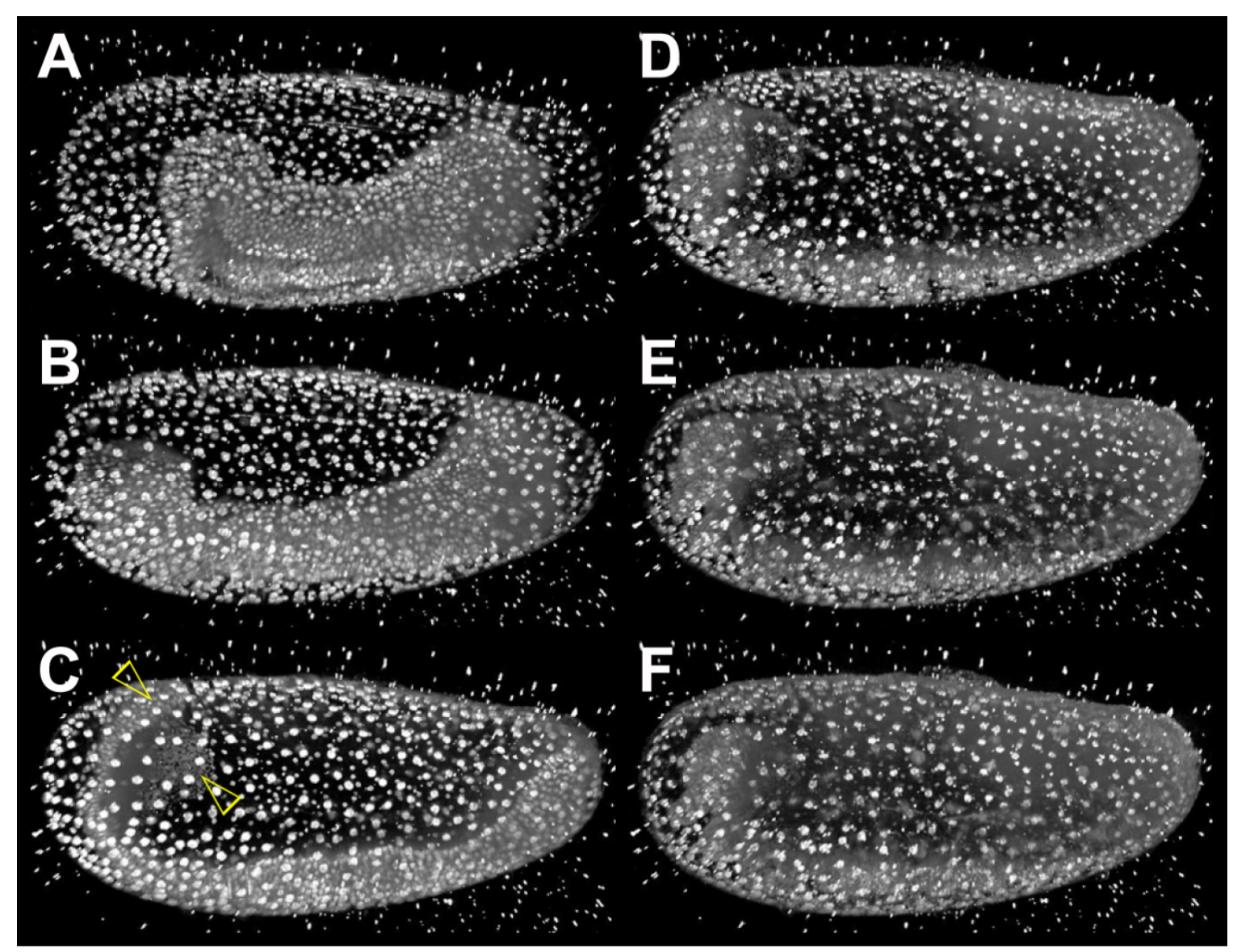

Figure 4.38 Early embryonic development imaged with the transgenic $\alpha$ Tubulin1P-H2Av::EGFP line in combination LSFM technique II. Anterior is left. The embryo was simultaneously imaged from three different angles. The embryo is depicted as rendered 3D projection. Spotty signals, outside of the embryo, are resulting from fluorescent beads, which are added to the mounting medium. These beads are used as landmarks to reconstruct the embryo by registration and fusion of the three separately imaged angles into one. (A-F) The imaging of three different angles and the subsequent reconstruction of the data by beadbased registration and rendering allows having a look through the entire embryo (C: empty arrowheads). 


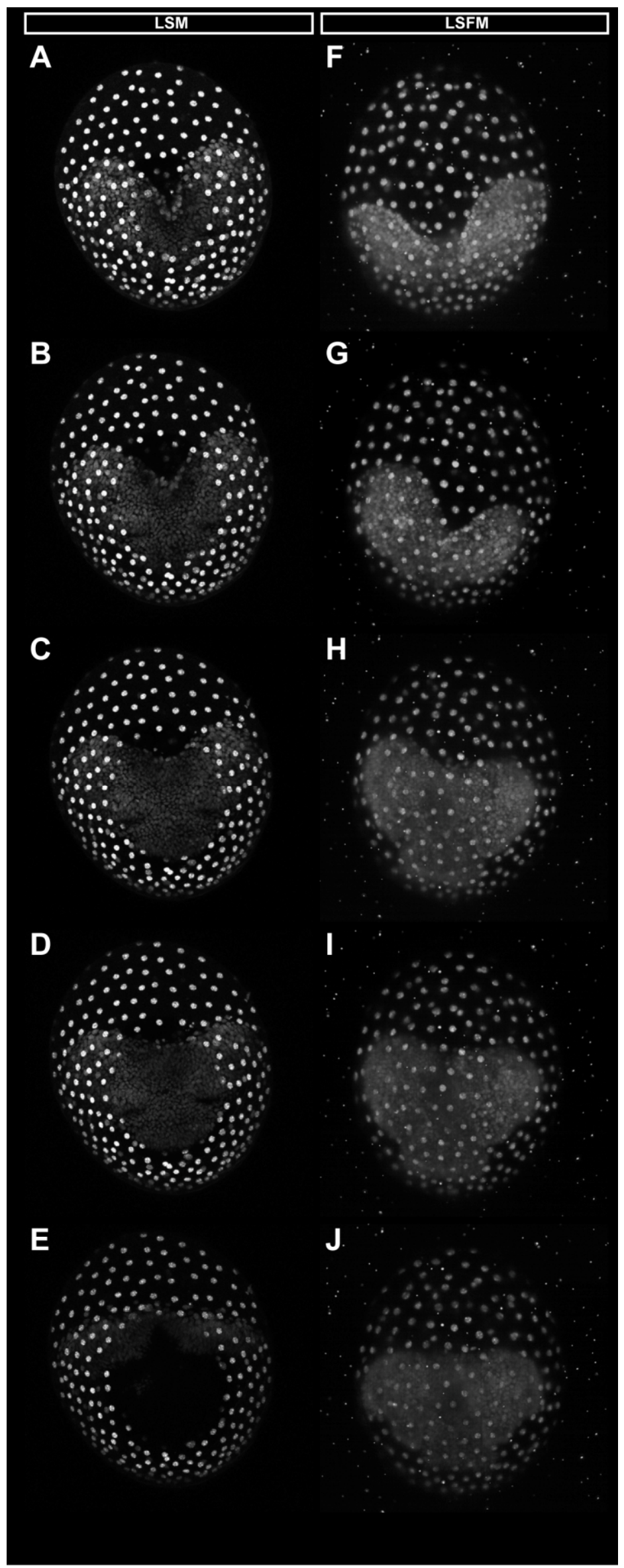

Figure 4.39 Comparison between

conventional LSM and LSFM imaging of the anterior embryonic cap. Anterior is up. Nuclei are visualized by using the transgenic aTub1P-H2Av::EGFP line and imaged either by laser scanning microscopy (A-E, LSM) or light-sheet-based fluorescence microscopy (F-J, LSFM) imaging techniques. The embryos are depicted as maximum projections. (AE) The movement of the anterior head from the ventral sub-terminal position of the egg to the anterior cap impedes tracing of the complete embryonic head development until larval stages via conventional LSM microscopy. (F-J) A proof of principle experiment shows that the possibility of different imaging views could be also exploited trying to trace the complete embryonic head development. The embryo was imaged from three different frontal views (1. ventrofrontal, 2. frontal, and 3. dorsofrontal). 


\subsubsection{Generation of cell marking lines for tracking experiments to}

\section{assemble an exact head fate map}

In order to get a better understanding of the morphogenetic movements of the head, I wanted to generate stable transgenic lines, which allow non-invasive cell marking and tracking. With this new tool it would be possible to precisely track down the movement of cells from early embryonic to L1 larval stages. This information would allow to assemble an exact head fate map and to get information about the cellular basis of head defects in loss-of-function or gain-offunction experiments. I tried to generate two different cell marking systems. (1) I wanted to generate a targeted laser-induced cell marking system on the basis of a photoactivatable fluorescent protein. This system allows transient but precise marking of single cells or small cell groups. Laser-induced cell marking in Tribolium, has so far only been shown by injecting mRNA of the actin-binding peptide fused to the photoconvertible Eos fluorescent protein (Benton et al., 2013; |zeddin et al., 2011) into embryos. Drawbacks of this transient system are that (I) the mRNA synthesis is expensive in the long-term, (II) embryonic injection is time-consuming, and (III) the method is invasive and could interfere with normal development. The generation of stably transgenic lines that allow laser-inducible cell marking would help to overcome these obstacles. (2) In addition, I wanted to generate a cell marking system on the basis of genetically marked cell clones, which lead to a random but permanent marking of small cell groups, which can be then traced throughout development. To this end, the Cre/loxP based brainbow cell marking system (Livet, 2007) for permanently genetic cell marking was already established in Tribolium (Averof, pers. communication). However, the system was so far suffering from low numbers of marked cells, and weak signal intensity of these marked cells (Bucher pers. communication). On this basis, I also wanted to exploit the Cre/loxP system (Metzger, 1999; Sternberg and Hamilton, 1981), with the following modifications concerning the responder line: (I) Use of another, more active ubiquitous promoter (instead of EFA); (II) Use of a stronger fluorescent marker protein (instead of m-RFP, m-YFP, and m-CFP); (III) Use of only one loxP flanked marker cassette (instead of IoxP, IoxN, and lox2272); (IV) Use of a fluorescent body marker expressed in unmarked cells (instead of no body marker). 


\subsection{Photoactivatable GFP lines for precise cell marking}

For the transient laser-induced cell marking system, I decided to use the photoactivatable fluorescent protein 'Cycle 3' mutant of GFP (C3PA-GFP; Ruta et al., 2010). The C3PA-GFP is an advancement of the first generation of photoactivatable-GFP (PA-GFP; Patterson, 2002) and shows a better and more stable signal after photoactivation (Ruta et al., 2010). These photoactivatable GFPs are mutants of the wt GFP (Tsien, 1998), marked by several amino acid substitutions. These substitutions lead to an altered, non-fluorescent confirmation of the GFP molecule. Upon laser treatment (appr. $400 \mathrm{~nm}$ ) the molecule undergoes a conformational change leading to strong fluorescence after excitation (appr. $488 \mathrm{~nm}$; Patterson, 2002). I generated two different transgenic lines with C3PA-GFP as fluorescent marker. Both lines drive ubiquitous expression under the control of the Tc- $\alpha$ Tub1 promoter. However, for one line I generated a chimeric C3PA-GFP, which is histone-tagged (H2Av::C3PA-GFP) and in consequence nuclear localized, whereas the other line carries the untagged cytosolic C3PA-GFP version.

\subsection{Photoactivatable GFP lines - Proof of principle}

I was able to generate several independent lines for both variants of the transgenic lines ( $\alpha$ Tub1P-C3PA-GFP $(\mathrm{n}=6)$ and $\alpha$ Tub1P-H2Av::C3PA-GFP $(\mathrm{n}=7)$ ). Afterwards, I tested all lines for functionality at embryonic stages. I collected eggs (0-24 h AEL) of all lines (pool of hetero- and homozygotes), and performed photoactivation via LSM for several times in an arbitrarily chosen region of the embryo. Before photoactivation and after each activation cycle I imaged the embryos to analyze changes regarding the fluorescent signal. The results of these lines showing the most efficient photoactivation capacity are shown in Figure 4.40A-D … Embryos of the two independent $\alpha$ Tub1P-C3PA-GFP lines ( $\alpha$ Tub1P-C3PA-GFP ${ }_{1 \_1}$ and $\alpha$ Tub1P-C3PA-GFP $2_{2_{-}}$) showed a considerable activation of the cytosolic fluorescent signal (Figure $4.40 \mathrm{~A}-\mathrm{B}^{\prime \prime \prime}$ ) already after the first cycle of photoactivation within the activated region (Figure 4.40A, B: dashed circles). However, the $\alpha$ Tub1P-C3PA-GFP ${ }_{23}$ line showed a better signal-to-noise ratio after each photoactivation cycle (Figure 4.40, compare $A^{\prime}$ and $B^{\prime}$ ). The two independent transgenic lines carrying the histone-tagged C3PA-GFP ( 2 Tub1P-H2Av::C3PA-GFP ${ }_{w 7}$ and aTub1P-H2Av::C3PA-GFP w5 ) showed a weaker photoactivation capacity (Figure 4.40C-D '.") compared to the lines with the untagged C3PA-GFP (Figure $4.40 A-B^{\cdots \cdots}$ ). Furthermore, the experiment showed -with the used laser lines ( $351 / 361 \mathrm{~nm}$ ) and settings (see section 3.12) - that the signal of the $\alpha T u b 1 \mathrm{P}-H 2 A v:: C 3 P A-G F P$ lines unexpectedly was localized in the cytosol but not 
in the nucleus (Figure 4.40C'”: yellow box). However, Anna Gilles (Averof lab, IGFL in Lyon (France)) was able to reveal, with a different laser line $(405 \mathrm{~nm})$, that the lines show a nuclear localized fluorescent signal upon photoactivation (Figure 4.40E).

Additionally, I was asking whether photoactivation is also feasible at larval and pupal stages. To this end, I performed a proof of principle experiment using all independent lines of aTub1P-C3PA-GFP and $\alpha$ Tub1P-H2Av::C3PA-GFP (data not shown). The analysis revealed that only the lines expressing the cytosolic C3PA-GFP showed a detectable photoactivationdependent fluorescent signal (data not shown). The line $\alpha$ Tub1P-C3PA-GFP $2_{-3}$ showed the best results, with a clear photoactivation-dependent fluorescent signal at larval and pupal stages (Figure 4.41A-B'). However, the signal was not uniformly distributed indicating an uneven distribution of cells or uneven expression (Figure 4.41A', B').

Taken together, these proof of principle experiments show that the transgenic laser-inducible cell marking lines are functional concerning their photoactivation capacity as well as the localization of the activated fluorescent signal. However, first long-term experiments revealed that the embryos were arrested in development upon photoactivation at 350-400 nm.

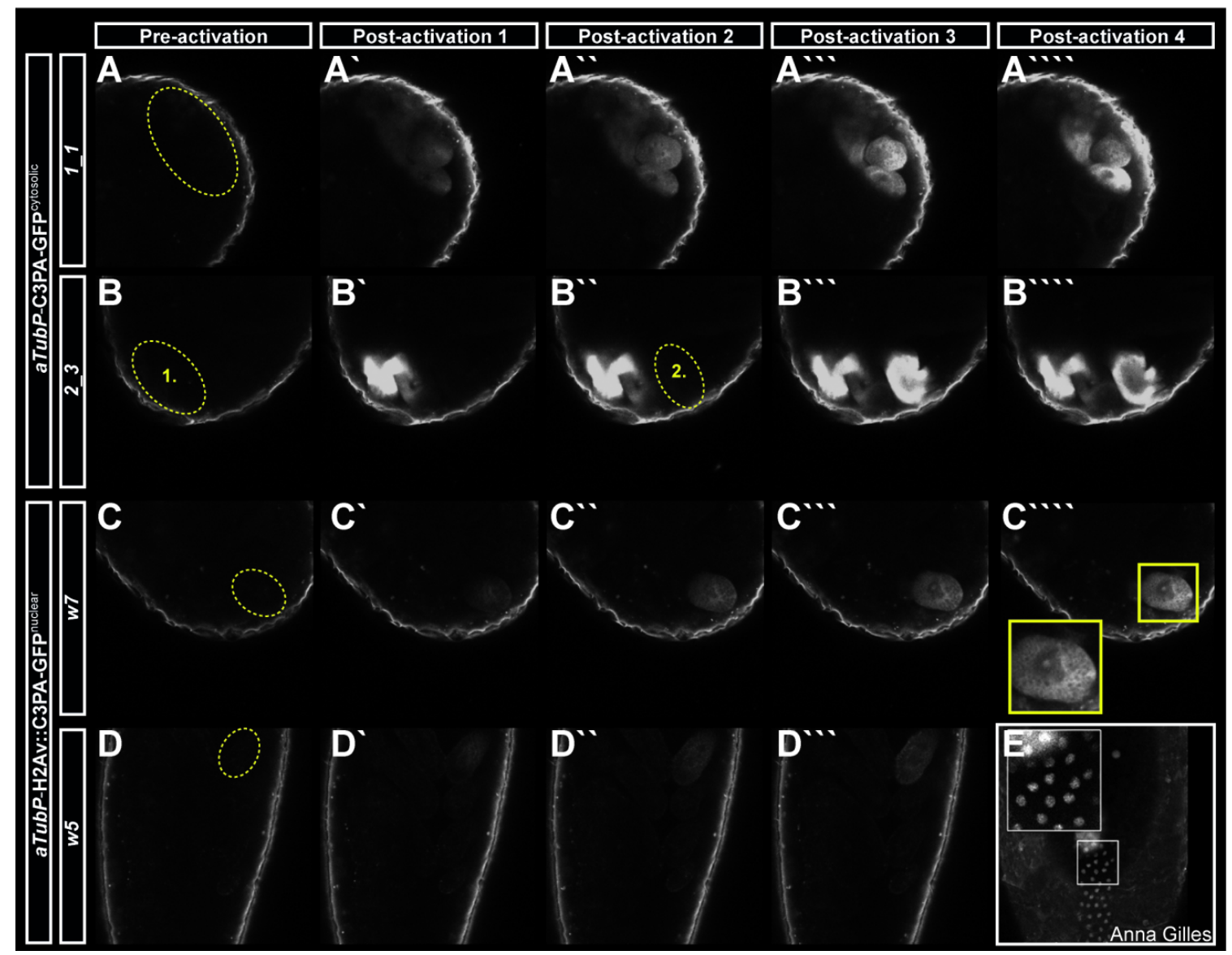


Figure 4.40 Test of C3PA photoactivation in different transgenic lines. Depicted are embryos from two independent lines, carrying the $\alpha$ Tub1P-C3PA-GFP construct (A-B $\left.{ }^{\cdots \cdots}\right)$, and two independent lines carrying the $\alpha$ Tub1P-H2Av::C3PA-GFP construct (C-E), before (A, B, C, D) and after photoactivation. Regions of photoactivation are marked by dashed circles. (A, B, C, D) Before photoactivation no C3PA-GFP signal is

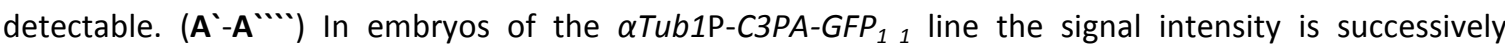
increases after each cycle of photoactivation. ( $\mathbf{B}^{\prime}-\mathbf{B}^{\cdots \cdot \cdots}$ ) Embryos of the $\alpha$ Tub1P-C3PA-GFP ${ }_{23}$ line show already strong fluorescent signal intensity after one cycle of photoactivation (B': ROI 1 and B'": ROI 2).

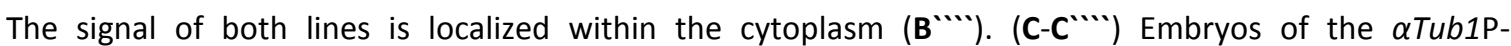
H2Av::C3PA-GFP ${ }_{w 7}$ line show a weak, but increasing, signal intensity after each cycle of photoactivation. (D-D '.) Embryos of the $\alpha$ Tub1P-H2Av::C3PA-GFP ${ }_{\text {w5 }}$ line show the weakest photoactivation capacity. With the used laser line $(351 / 364 \mathrm{~nm})$ and settings nuclear localization of the signal could not be obtained (C'”: box). However, Anna Gilles was able with the same transgenic H2Av::C3PA-GFP line, but a different laser line (405 nm), to get a nuclear localized signal upon photoactivation (E: box).

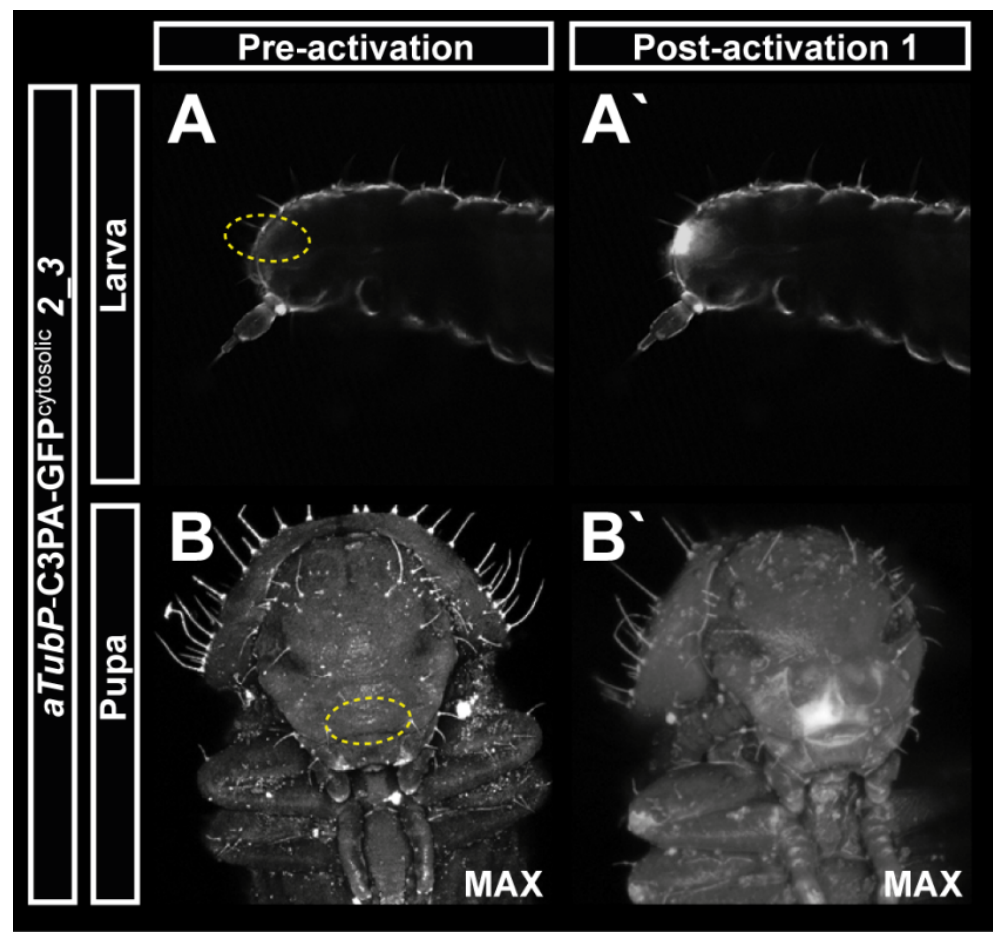

Figure 4.41 Test of C3PA photoactivation capacities at larval and pupal stages. Depicted is a larva, in single plane section $\left(\mathbf{A}, \mathbf{A}^{\prime}\right)$ and a pupa, as maximum projections (B, $\left.\mathbf{B}^{\prime}\right)$ from the line $\alpha$ Tub1P-C3PA-GFP $2_{3}$ before $(\mathbf{A}, \mathbf{B})$ and after photoactivation $\left(\mathbf{A}^{\prime}, \mathbf{B}^{\prime}\right)$. Regions of photoactivation are marked by dashed circles. (A, B) Before photoactivation no C3PAGFP signal is detectable. (A-B') Photoactivation of the C3PA-GFP in larvae and pupae of the transgenic line $\alpha T u b 1 P-C 3 P A-G F P_{23}$ is feasible, but shows an uneven signal distribution. 


\subsubsection{Responder line for a genetic cell marking system}

Embryonic development of Tribolium takes several days (at $32^{\circ} \mathrm{C}$ about three days; (Brown et al., 2009)). It is questionable whether the amount and photo-stability of the photoactivated molecules will be high enough to image the development repeatedly for a longer period of time. Trying to overcome this potential drawback of the photoactivation based cell marking system, I also generated transgenic responder lines, which are able, with the adequate driver line, to genetically and therefore permanently mark cells. For this purpose I exploited the Cre/loxP system (Metzger, 1999; Sternberg and Hamilton, 1981). The driver line was already established in my lab (unpublished). This transgenic driver line is expressing the Cre (causes recombination) recombinase under the control of the Tc-hsp68 promoter. Upon heat shock treatment the Cre recombinase is ubiquitously expressed. However, stochastic distribution of Cre expressing cells and level-dependent activity of the Cre recombinase lead only in a small subset of cells to excision events of loxP (locus of crossing over (x), P1) flanked sequences (Bucher, pers. communication). My aim was to generate a transgenic loxP responder line (Figure 4.42). In the off-state, the loxP responder line should drive ubiquitous expression of cytosolic monomeric Cherry (mCherry, red flourescent protein; Figure 4.42A) as body marker. After crossbreading with the driver line and heat shock treatment cells should express the Cre recombinase (Figure 4.42B, C). In consequence, cells expressing the Cre recombinase should stop the mCherry expression and start the expression of H2Av::EGFP, due to a cyclization and excision event of the loxP-flanked cassette (Figure 4.42C, D, E). Thus, a small cell population should be marked by a green nuclear localized signal (Figure 4.42E), whereas the unmarked cells should still express mCherry marked by a red cytosolic signal (Figure 4.42A). 


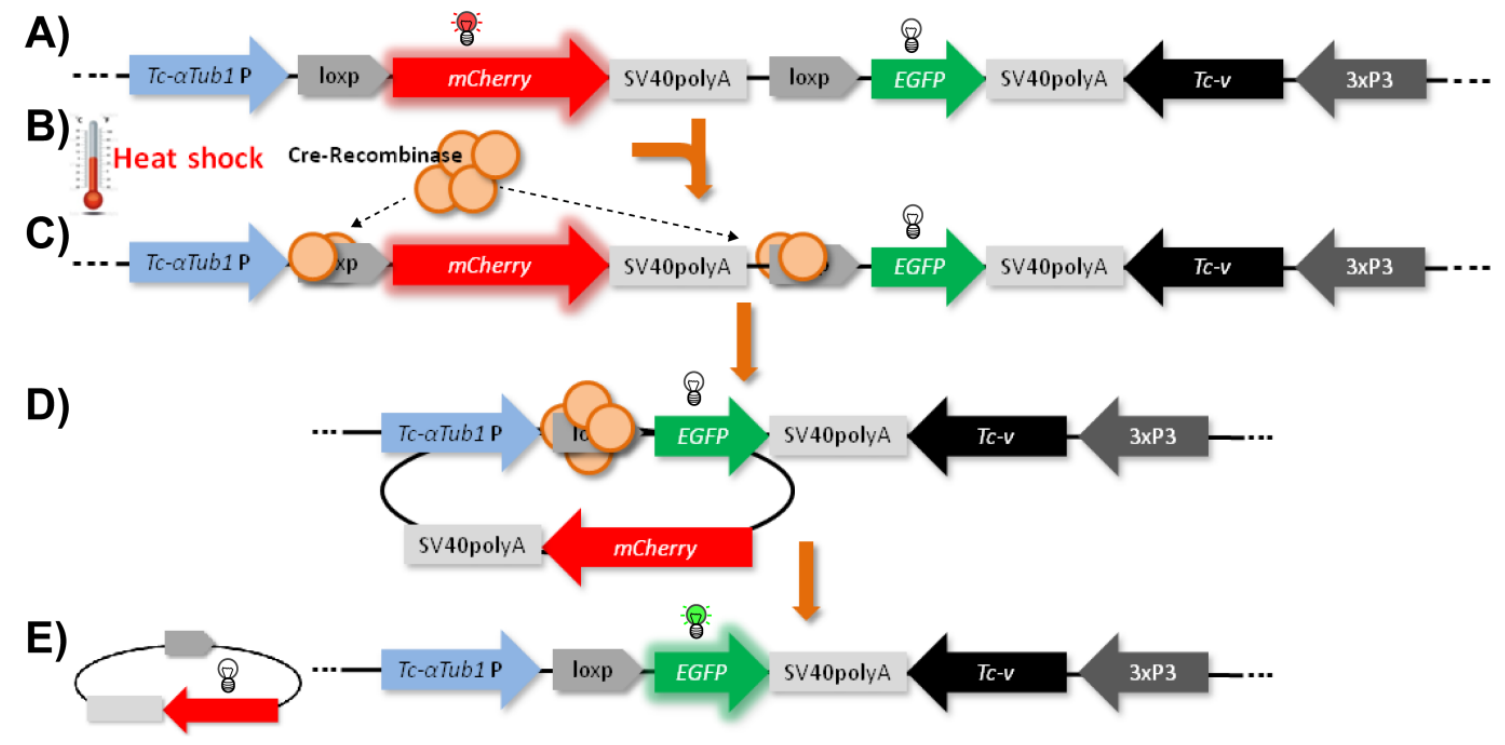

Figure 4.42 Scheme of the genetic cell marking system exploiting the Cre/loxP system. (A) In the off state, the loxP responder line carrying the ubiquitous promoter (Tc- $\alpha T u b 1 \mathrm{P})$, which drives ubiquitous expression of the red body marker (mCherry, cytosolic). The body marker is flanked by two loxP sites, which are oriented in the same direction. The body marker cassette is followed by the green cell clone marker (EGFP, histone-tagged for a nuclear localization), which is not expressed in the off state. The transgenesis marker $(T c-v)$, which is driven by an eye-specific promoter, rescues the eye color in transgenic individuals within the $T c-v^{w}$ strain (white eyes switch to black eyes). The Cre driver line drives expression of the Cre recombinase, upon heat shock treatment (not depicted here). (B) Crossing of the loxP responder line with the Cre driver line and the subsequent heat shock treatment of the offspring leads to random expression of Cre recombinase. (C) The Cre recombinase enzyme binds to the loxP sites. (D) Due to the orientation of the loxp sites, the Cre recombinase starts the cyclization and excision of the loxP-flanked sequence. (E) The excision abolishes expression of the body marker, but induces expression of the green cell clone marker. Thus, cells will be marked by a green signal within the nuclei.

\subsubsection{Genetic cell marking system - Proof of principle}

In order to test whether the generated loxP responder lines are functional, I performed two proof of principle experiments. The first experiment should reveal whether the uncrossed loxP responder line shows only the red body marker signal for unmarked cells. The second experiment should show whether, in offspring of the crossbred driver and the responder lines, a small population of cells is marked by a green signal upon heat shock treatment. To this end, I collected eggs (0-48 h AEL) from the loxP responder line and from crossbreds of the independent loxP responder lines and the Cre driver line. Offspring were either untreated (neg. control) or treated with one heat shock. The individuals were analyzed the following day. 
Analysis of the offspring of the uncrossed independent loxP responder lines revealed that most of the lines showed the red body marker $(n=4 / 6)$ but no green signal (data not shown) as expected. Heat shock treatment of the crossbred offspring (Cre driver $x$ loxP responder lines) led to marked cells showing a green signal in all four lines (result of the independent line aTub1P-Lox(mcherry);H2Av::EGFP 7 as representative: Figure 4.43A-B"'). Further, the signals were correctly localized. The red signal was, although weak and granular, localized to the cytosol (Figure 4.43A, B) and the green signal was nuclear localized (Figure 4.43A', B': white box). Both signals could be traced in embryos (Figure 4.43A", C") as well as in L1 larvae (Figure 4.43B", D"). However, the number of genetically marked cells was very high (Figure 4.43A", B"). Asking whether those embryos that received no heat shock treatment, showed a lower number of marked cells, I analyzed them regarding the number of cells expressing H2Av::EGFP. The analysis revealed that also the untreated individuals showed a very high number of marked cell clones (Figure 4.43C-D").

In summary, the generated loxP responder lines are functional and are able to respond to the Cre expression of the driver line. However, the number of marked cells was very high, even without heat shock treatment, and therefore not suitable for cell tracking and fate-mapping experiments. 


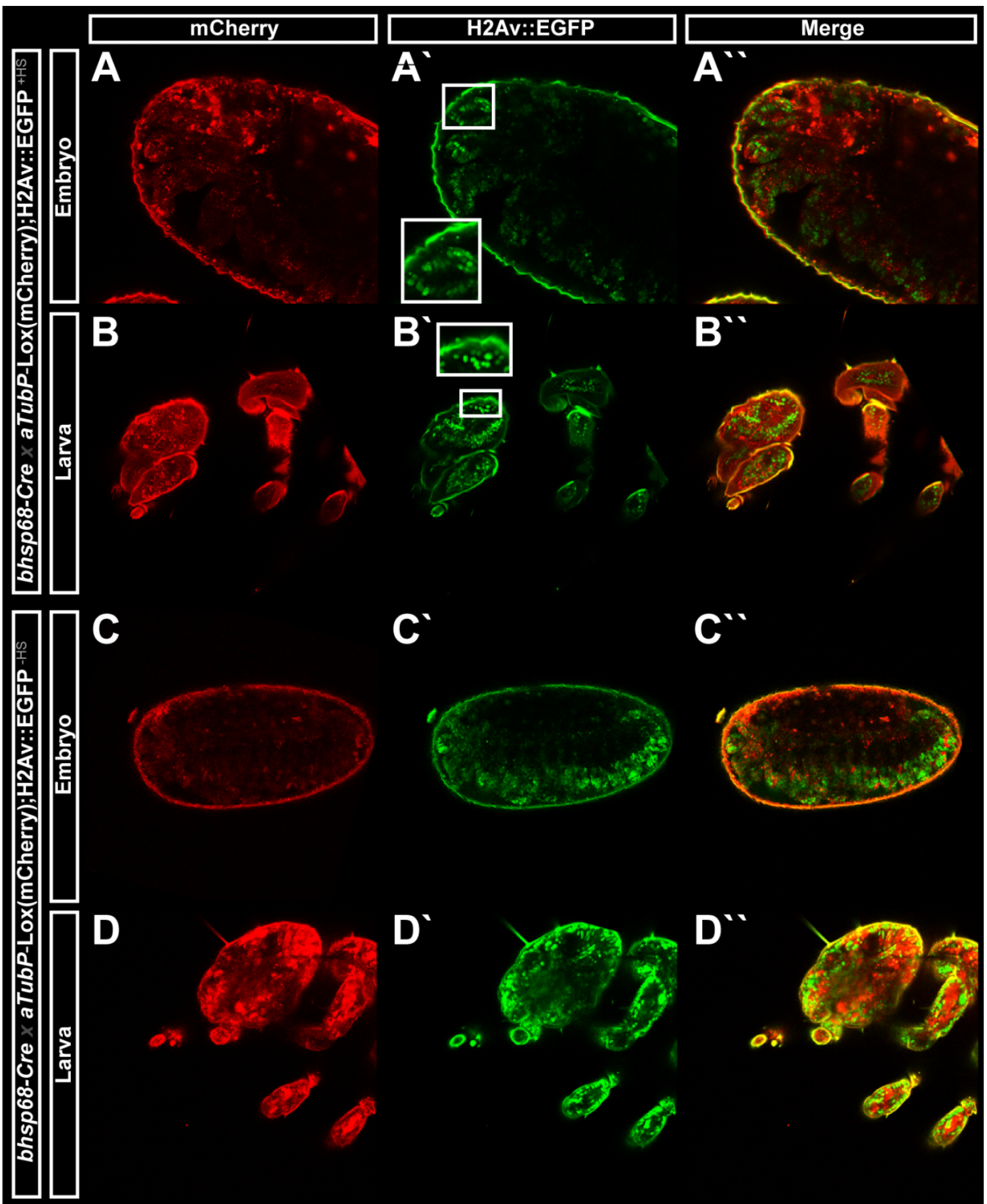

Figure 4.43 Test of the genetic cell marking system. Depicted are embryos and larvae from the crossbreeding of the hsp68-Cre driver and the $\alpha$ Tub1P-Lox(mcherry);H2Av::EGFP, responder line, which were either heat shocked during embryonic stages (0-48 h AEL; A-B “) or remained untreated (C-D“). (AA") Embryos of the crossbred show a weak but ubiquitous mCherry expression (A). Further, a subset of cell clones is marked by the expression of H2Av::EGFP (B) which is nuclear localized (white box). These findings suggest functionality of the loxP responder line and the cell marking system. However, the number of marked cell clones is very high. (B-B") The body marker and marked cell clones are detectable 
also at larval stages. (C-D') The number of marked cell clones $\left(\mathbf{C}^{\prime}, \mathbf{D}^{\mathbf{}}\right)$ is also very high in crossbred offspring, which were not heat shocked. 
5

\section{Discussion}

\subsection{Tc-foxq2 is required for head and brain development}

\subsubsection{Significance of the study}

foxq2 was shown to be expressed at the anterior pole in many metazoan species. Within arthropods, foxq2 expression was so far only reported for Drosophila and Strigamia, being located at the anterior pole, as well. Furthermore, foxq2 was suggested to form together with six3 a conserved core regulatory module, which is required for anterior patterning. So far functional data was only reported for the deuterostome Strongylocentrotus with having a function in ectodermal and neural development, and for the cnidarian Nematostella with having a function in neural development as well. However, data on foxq2 function in protostomes was completely missing. In this work, I was able to show that foxq2 function is required for proper epidermal and neural development. Further, I was able to reveal a comprehensive gene regulatory network that shows that foxq2 is acting -together with six3- as a core regulatory module for anterior head development.

\subsubsection{Gene regulatory network of anterior head development}

One of the main aims of this study was to identify the function of $T c-f o x q 2$ in the gene regulatory network responsible for pattering the anterior head. This study revealed that $T c$-foxq2 expression is localized at the apical pole (Figure 5.3D), and is antagonized by Wnt/ß-catenin signaling (Figure 5.1), as expected from previous data. Its function is essential for the correct formation of the anterior ectoderm. On the basis of the expression pattern of Tc-foxq2, coexpression studies with head patterning genes as well as knock-down and gain-of-function studies I integrated $T c$-foxq 2 into the already established interaction network (Based on Kittelmann et al., 2013; Posnien et al., 2011b; Siemanowski et al., 2015). The hierarchal order of the genes and their interactions shown in Figure 5.1 were based on: 1. Expression onset, 
2. time/region of co-expression, 3. expression data gathered from knock-down/gain-of-function experiments, and 4. extent of defects in L1 larvae after knock-down. It is noteworthy that both cell death rate and cell proliferation showed no significant differences during early patterning of the anterior head in $T c-f o x q 2^{\text {pRNAi }}$ embryos compared to wt embryos. Therefore, the early effects of $T c$-foxq 2 on the head patterning genes are very likely primary regulatory interactions and not secondary effects due to tissue loss. My data revealed that $T c$-foxq2 is a key upstream factor in pattering the anterior ectoderm and neuroectoderm (Figure 5.1).

I was able to show that $T c$-foxq 2 and $T c$-six 3 are forming a core regulatory unit responsible for patterning the anterior head. This finding is based on the fact that both show similar epidermal and neural phenotypes, in knock-down experiments. Further, Tc-foxq2 is co-expressed with $T c$-six3 at the anterior pole, at most developmental stages (Figure 5.4). Both together share an upstream position in the anterior head gene regulatory network and show a highly similar regulation of several downstream targets. Moreover, $T c$-foxq2 and $T c$-six 3 are regulating each other mutually from early stages onwards. This is similar to the eye network where mutual activation by eyeless, eyes absent, dachshund, twin of eyeless, and sine oculis is required for eye development and where mutations in either of those genes lead to loss of eyes (Wagner, 2007).

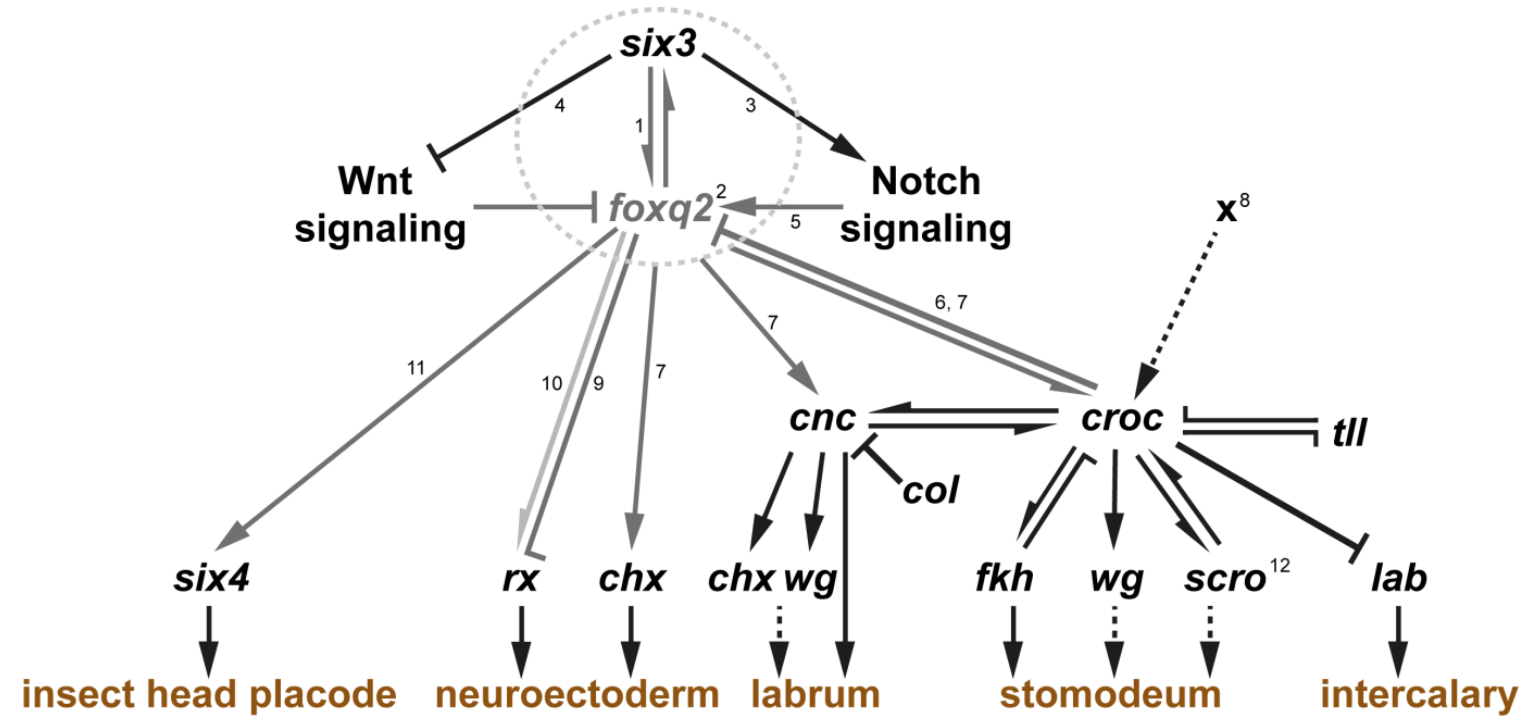

Figure 5.1 Tc-foxq2 is an upstream player within the gene regulatory network of the anterior Tribolium head. Black lines indicate previously reported parts of the network (Based on Kittelmann et al., 2013; Posnien et al., 2011b; Siemanowski et al., 2015). Grey lines represent new data based on Tc-foxq2 experiments. Arrows represent gene activation, and cross-bars gene repression. Dashed lines indicate hypothetical effects. (1) Tc-six3 is the major factor for patterning the anterior head due to the early onset of expression and the strongest knock-down cuticle phenotypes with epidermal defects spanning the 
labral to the ocular regions and neural defects marked by a loss of the central body, defective mushroom bodies and fused brain hemispheres. (2) $T c$-foxq2, like $T c$-six3, is a key player in anterior head development with a somewhat later onset of expression than $T c-s i x 3$, but similar cuticle phenotypes (epidermal \& neural), and comparable activities in patterning of the anterior head. Mutual activation and similar phenotypes suggest that they form a regulatory module (indicated by a dashed circle). (3) Tc-six3 acts on Notch signaling via Tc-ser. However, it is not clear whether the effect is primary or secondary. (4) Tc-six3 prevents the ocular Tc-wg domain from expansion into the AMR, but is not acting on other $T c$-wg domains. (5) Notch signaling-dependent activation of $T c-f o x q 2$ is only partial and restricted to lateral parts of the anterior median $T c$-foxq2 domains (Tc-mib1 data). (6) Tc-foxq2 like $T c$-six3, only regulates the anterior (ectodermal) part of the Tc-croc expression domain. (7) Regulative activity on several downstream targets is essentially indistinguishable between $T c$-foxq2 and $T c$-six3 knock-down experiments. (8) An unknown factor ' $X$ ' is predicted to regulate the posterior (mesodermal) part of the $T c$-croc expression, because $T c$-six 3 and $T c$-foxq2 regulate only the anterior (ectodermal) portion of the $T c$-croc AMR domain $(6)$. $(9,10)$ Results of $T c$-foxq2 gain-of-function and knock-down experiments are conflictive. Since, $T c-r x$ is repressed when $T c-f o x q 2$ is ectopically expressed (9), simultaneously $T c-r x$ expression vanishes in $T c$-foxq2 knock-down experiments (10), indicating activation by $T c$-foxq2. However, in the relevant stages both genes are mutually exclusively expressed, which argues against direct activation (10, indicated by light grey arrow) and points to interaction via diffusible signaling molecules. (11) Data for interaction of Tc-six3 with Tc-six4 is missing, and thus it is impossible to judge whether it is regulated by the $T c-f o x q 2 / T c-s i x 3$ regulatory module so far (dashed line). (12) The late effect of $T c-f o x q 2$ on $T c$-scro, observed in gain-of-function experiments, is most likely secondary and is, hence, not considered.

\subsubsection{Late $T c$-foxq2-associated effects}

The genetic interaction studies on $T c$-foxq 2 do not only reveal alterations in the expression pattern and morphology at early embryonic stages, but also at stages from limb formation onwards.

Figure 5.2 gives an overview of the later effects of $T c$-foxq2 knock-down. However, most of these interactions could not be clearly determined as being a primary or secondary effect. This is due to the fact that in knock-down experiments most of the genes at these stages show an altered cell death rate, cell proliferation rate or tissue malformation (Figure 4.5, Kittelmann et al., 2013; Posnien et al., 2011b; Siemanowski et al., 2015). 


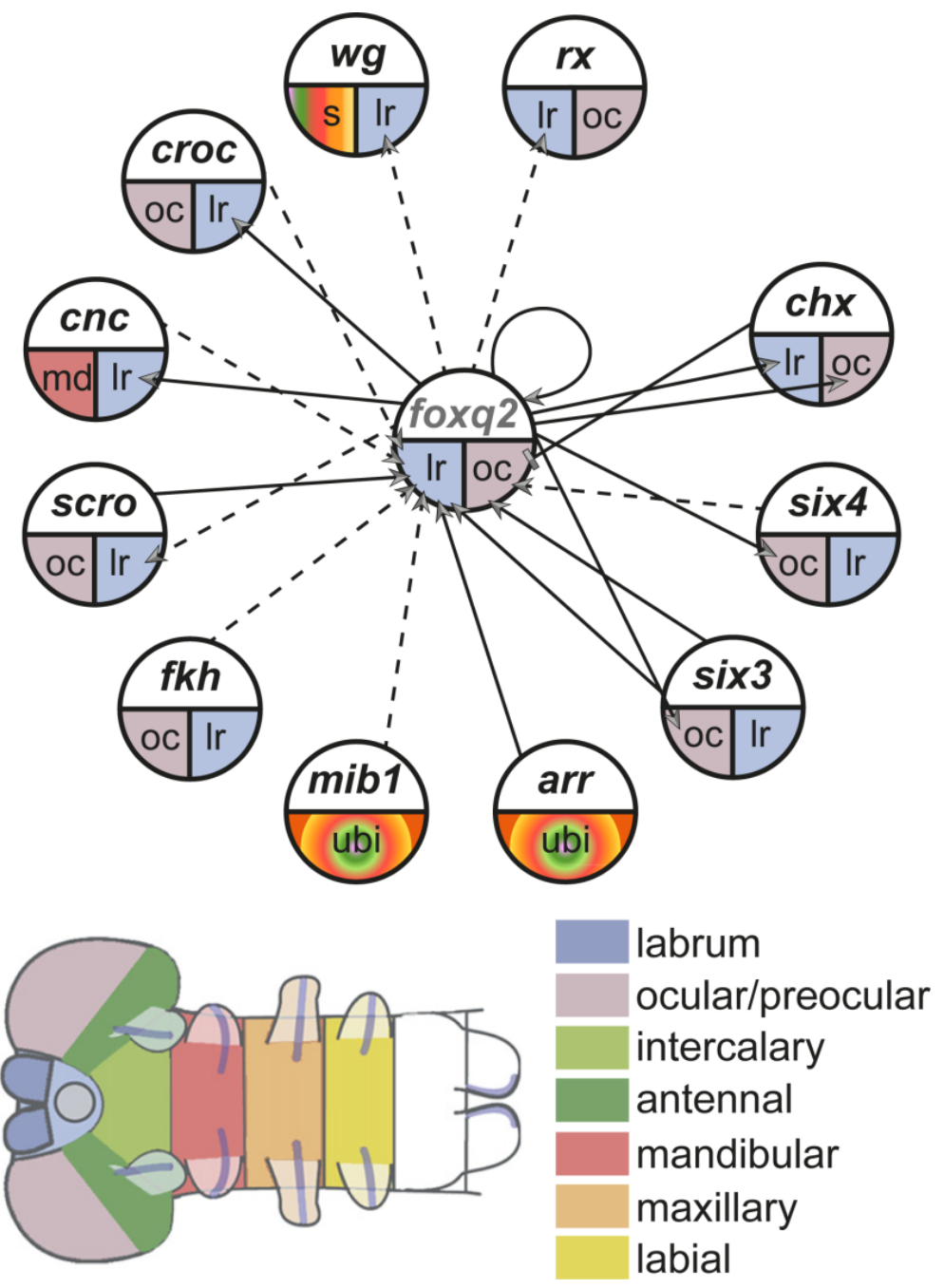

Figure 5.2 Late effects of and on Tc-foxq2. The upper part shows the non-hierarchical interaction network at late embryonic stages. Each circle shows in the top part the gene name and in the bottom part the regions, in which the respective gene shows expression. The continuous lines indicate interactions, which are most probably primary. Dashed lines indicate interaction, which could be primary or secondary. Ends of the lines indicate activation (arrowheads) or repression (crossbar) and point to the affected expression domain. The lower part of the figure serves as legend demonstrating the location of the affected region (Embryonic scheme taken from Posnien and Bucher, 2010). Ir: labrum, md: mandibular, oc: ocular/preocular, s: segmental, ubi: ubiquitous

\subsubsection{4 foxq2 and six 3 - a highly conserved signaling center for pattering the apical pole}

Representatives of the foxq2 subfamily were found in various species distributed over the metazoan kingdom (Chapman et al., 2010; Chevalier et al., 2006; Darras et al., 2011; 
et al., 2008; Lee and Frasch, 2004; Marlow et al., 2014; Martín-Durán et al., 2015; Martín-Durán and Hejnol, 2015; Mazet et al., 2003; Santagata et al., 2012; Shimeld et al., 2010; Sinigaglia et al., 2013; Tu et al., 2006; Yaklichkin et al., 2007; Yu et al., 2008, 2003; Zhang et al., 2014). The majority of these species show a well-conserved expression of foxq2 at the anterior/apical pole (Figure 5.3; Chevalier et al., 2006; Darras et al., 2011; Fritzenwanker et al., 2014; Hunnekuhl and Akam, 2014; Lee and Frasch, 2004; Martín-Durán et al., 2015; Santagata et al., 2012; Sinigaglia et al., 2013; Tu et al., 2006; Yu et al., 2003). Furthermore, some recent studies showed coexpression of foxq2 and six3 at the apical pole (Figure 5.4; Fritzenwanker et al., 2014; Hunnekuhl and Akam, 2014; Marlow et al., 2014; Martín-Durán et al., 2015; Santagata et al., 2012; Sinigaglia et al., 2013; Tu et al., 2006; Wei et al., 2009). So far mutual co-regulation for foxq2 and six3 was only shown in Strongylocentrotus. In Strongylocentrotus, six3 activates foxq2, while foxq2 is repressing six3 (Range and Wei, 2016; Wei et al., 2009; Yaguchi et al., 2010). Further, it was shown that both together form a conserved regulatory unit for pattering the anterior ectoderm, antagonized by Wnt/ß-catenin signaling (Darras et al., 2011; Fritzenwanker et al., 2014; Marlow et al., 2014, 2013; Range and Wei, 2016; Sinigaglia et al., 2013; Wei et al., 2009; Yaguchi et al., 2008).

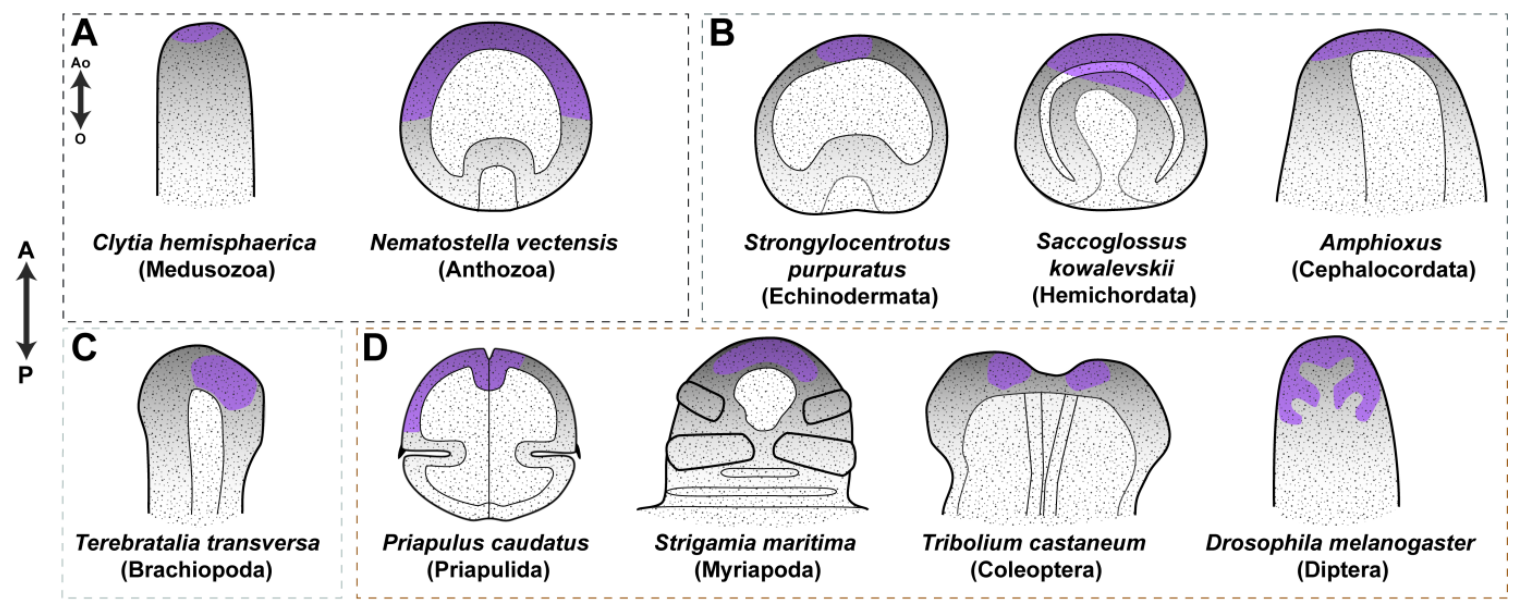

Figure 5.3 Conserved expression pattern of foxq2 in metazoan kingdom-spanning species. Schematic representation of foxq2 expression (purple) in cnidarians (A), deuterostomes (B), lophotrochozoans (C), and ecdysozoans (D). All species are oriented with the anterior/aboral pole to the top. Ectodermal expression of foxq2 at the most anterior part is highly conserved in different members distributed across the metazoan kingdom. (Based on Chevalier et al., 2006; Fritzenwanker et al., 2014; Hunnekuhl and Akam, 2014; Lee and Frasch, 2004; Martín-Durán and Hejnol, 2015; Santagata et al., 2012; Sinigaglia et al., 2013; Tu et al., 2006; Yu et al., 2003); A: anterior, P: posterior, Ao: aboral, O: oral 
I showed that foxq2 and six3 in Tribolium show the previously described co-expression pattern, characterized by largely overlapping expression domains of both factors (Figure 5.4, left column). However, in Tribolium elongating germ bands the expression of both factors becomes mutually exclusive to each other (Figure $5.4 \mathrm{H}^{\prime \prime}$ ), which so far has only been reported for late gastrulae of Strongylocentrotus (Figure 5.4B`; Range and Wei, 2016; Tu et al., 2006). Also other species show diversification of the foxq2/six 3 expression/co-expression pattern at later stages, ranging from a complete six3/foxq2 co-expression with a six3-free region at the most apical part (Figure 5.4A , E) to an absence of both factors in the most apical part, being only co-expressed in more posterior regions (Figure 5.4D', F). At later Tribolium embryonic stages co-expression of $T c$-foxq2 and Tc-six3 starts again, but with a quite complex expression profile compared to other species at later stages (Figure $5.4 \mathrm{H}^{\cdots}, \mathbf{H}^{\cdots \cdots}$ ). Taken together, at least at early stages the coexpression of foxq2 and six 3 appear to be the ancestral condition. From the distribution of coexpression it is even unclear what the ancestral condition was for later stages. In Nematostella and Platynereis both factors are co-expressed at later stages, while Strongylocentrotus, Terebratalia, Lineus, and Tribolium show only minor expression overlaps at later stages.

With respect to the co-regulation of both factors it is known that six 3 is repressed by foxq 2 in Strongylocentrotus (Range and Wei, 2016). In contrast, in Tribolium foxq2 and six3 both regulate each other positively forming an upstream regulatory module (see Figure 5.1). Due to the lack of functional data in other taxa statements about the ancestral condition remain impossible.

On the one hand, these data support the notion that the set of factors acting on the regulation of anterior tissue is well-conserved (Range and Wei, 2016; Sinigaglia et al., 2013). On the other hand, the exact expression dynamics, co-expression, and interactions of these conserved factors appear to have evolved substantially depending on the evolutionary situation within the metazoan kingdom, culminating in a complete loss of foxq2 representatives in the Placentalia. This differential regulation reflects a high degree of plasticity of these factors in evolutionary processes (Sinigaglia et al., 2013). This suggests that the core apical patterning gene set evolved to a significant degree and being involved in head shape diversification. This is similar to trunk patterning by Hox genes. The highly conserved Hox genes of the conserved Hox gene cluster play a crucial rule in AP patterning and building the body. However, evolutionary changes of these genes influence development and in consequence trunk morphology (Carroll, 1995; Lemons, 2006; Pearson et al., 2005). 
The importance of Hox genes is due to the fact that these genes are involved in many processes of development e.g. cell cycle control, cell adhesion, cell division rates, cell death, and cell movement (Pearson et al., 2005; Weatherbee et al., 1998). 

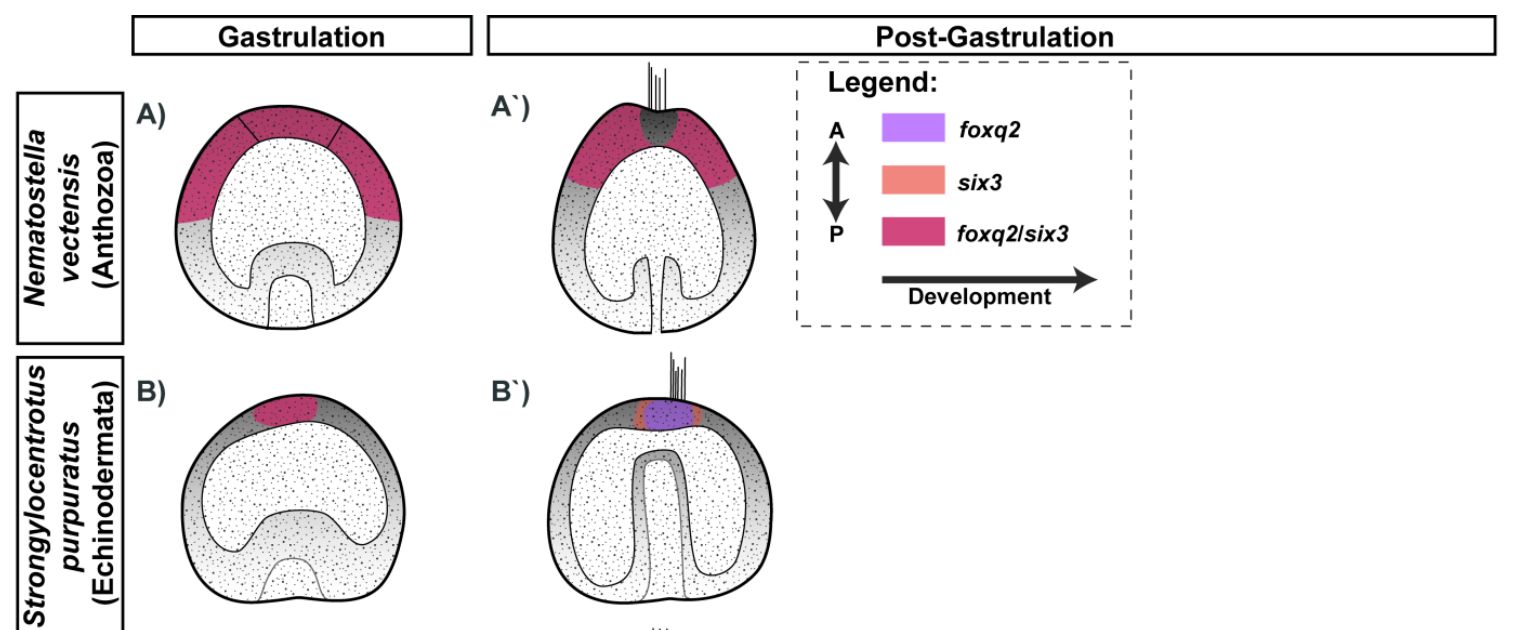

B')
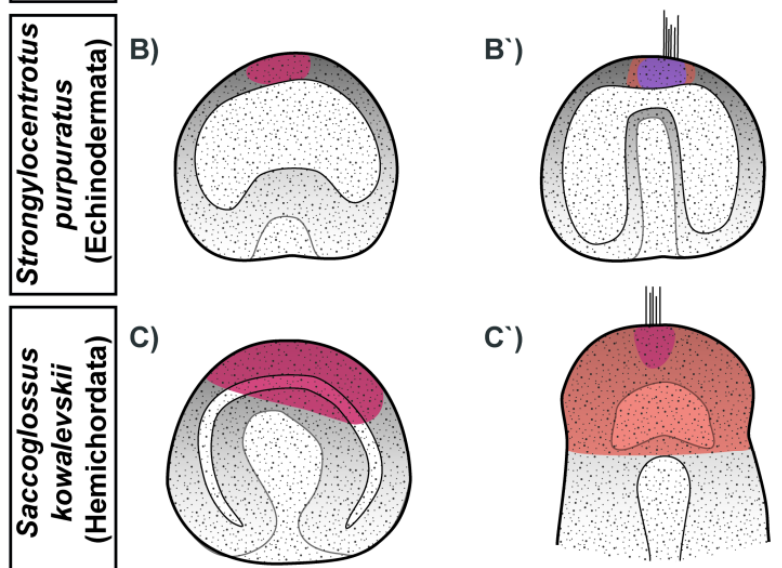

c)
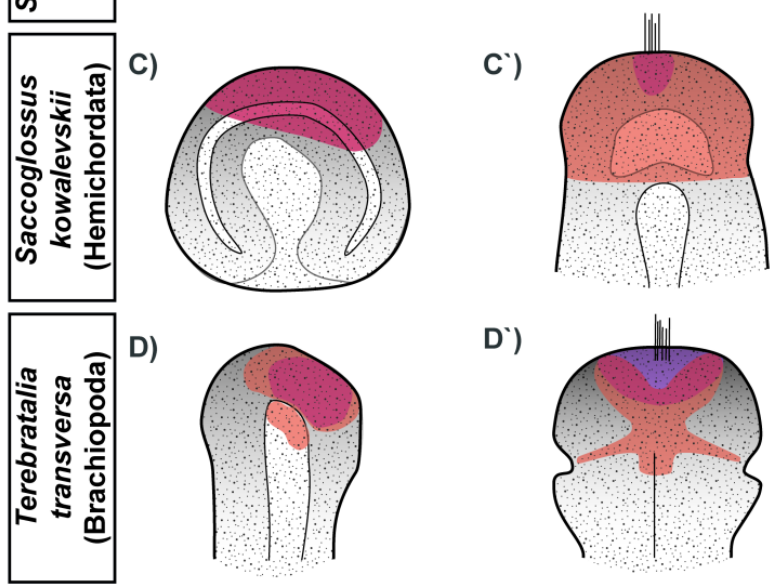

D')
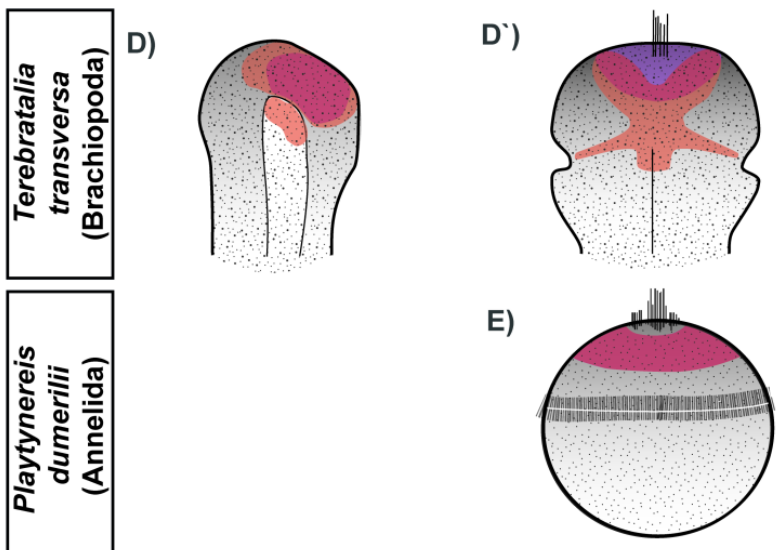

E)

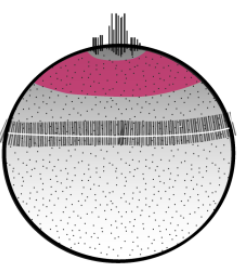

F)

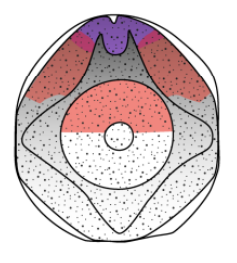

G)

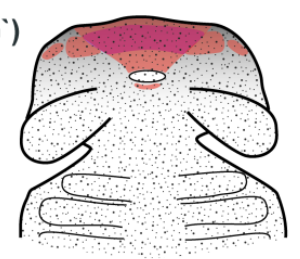

F)

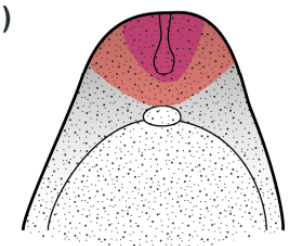

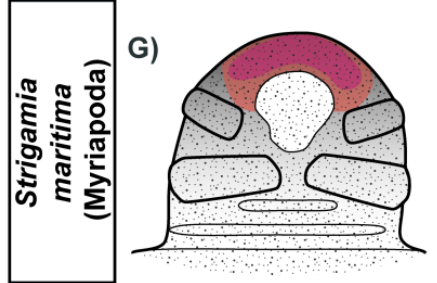
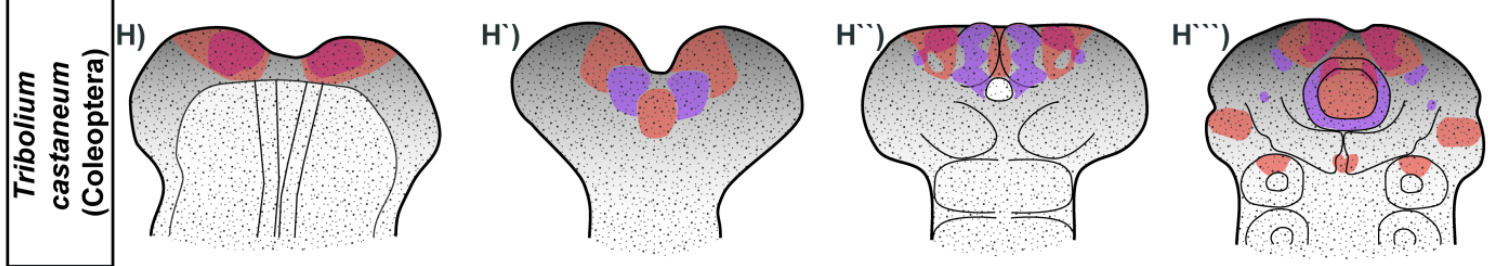
Figure 5.4 Co-expression of foxq2/six3 at different developmental stages of different Metazoa. Schematic representation of foxq2 (purple) and six3 (orange) expression in metazoan species at different developmental stages. Co-expression is marked in red. The anterior/apical pole is oriented to the top. Expression/co-expression of foxq2 and six3 at early stages of different metazoan species is highly conserved marked by largely overlapping domains at the anterior/apical pole (left column). At later stages the expression/co-expression of both factors diversifies. (A', E) Nematostella and Platynereis larvae show a foxq2/six3 co-expression during early stages like the other species, with the exception that the most apical region is free of foxq2/six3 expression. (C', $\mathbf{F}^{\prime}, \mathbf{G}^{\prime}$ ) Late embryonic stages of Saccoglossus and Strigamia as well as early Lineus juveniles show a foxq2 expression at the anterior/apical pole, which is completely covered by six3 expression. (B', H') Strongylocentrotus late gastrulae and Tribolium elongating germ bands show mutually exclusive expression of foxq2 and six3. (D', F') Early tri-lobed Terebratalia larvae and Lineus Schmidt's larvae show foxq2 expression at the anterior/apical pole overlapping only posteriorly with six3. (H“, $\left.\mathbf{H}^{\cdots}\right)$ Fully elongated and retracting Tribolium germ bands show a complex expression pattern of foxq 2 and six 3 with partial overlaps in the neuroectoderm $\left(\mathbf{H}^{*}, \mathbf{H}^{\cdots}\right)$ and in the anterior labral buds (H“)'). (Based on Fritzenwanker et al., 2014; Hunnekuhl and Akam, 2014; Marlow et al., 2014; Martín-Durán et al., 2015; Santagata et al., 2012; Sinigaglia et al., 2013; Tu et al., 2006; Wei et al., 2009); A: anterior, P: posterior

\subsubsection{Ectopic expression of Tc-foxq2}

Heat shock-based ubiquitous ectopic expression is a powerful tool for studying gene function due to the adjustable time point and strength of ectopic expression (Brand et al., 1994; Schinko et al., 2012). This method has been established previously but has not been applied so far. In this study, I generated a functional transgenic line for ubiquitous ectopic expression of $T c$-foxq2. The transgenic line in combination with expression studies provided a good complementary view on $T c$-foxq2 function in patterning of the anterior head region. Expression analyses showed that the majority of the head pattering genes are responding, if at all, only in certain regions to ectopic $T c$-foxq2 expression. This indicates that gene activation/repression of $T c$-foxq2 target genes is also dependent on a permissive environment, which is created by other activators or repressors acting on the particular gene. The pleiotropic effect of ectopic expression of the transcription factor Tc-foxq2 indicates that there are the same or further target genes, which respond to the presence of $T c-f o x q 2$ in tissue posterior to the anterior part of the head. This is also confirmed by the disrupted expression pattern of the segment polarity gene $T c-w g$. While the effect on the trunk and the appendages are reproducible it does not reflect biologically meaningful interactions because this occurs outside the $T c$-foxq2 expression domains. The results of the 
expression pattern analysis of head patterning genes were partially ambiguous in $T c$-foxq2 gainof-function experiments. In some cases the effect was not very pronounced or could not be clearly assigned to be a primary effect.

However, although heat shock-induced ubiquitous expression is a well-suited method for studying gene function it also has some drawbacks: the ectopic expression is ubiquitous and cannot be restricted to a subset of cells, and basal expression levels of the heat shock promoter are to be expected (Brand et al., 1994). In order to circumvent these disadvantages it would be helpful to generate and exploit specific GAL4 enhancer lines (Brand et al., 1994; Prelich, 2012; Schinko et al., 2010). It would be interesting to drive overexpression of head patterning genes in the $T c$-foxq 2 region and look for alterations in $T c$-foxq 2 expression. Moreover, reciprocal Tc-foxq2 ectopic expression in the pattern of other head patterning genes and subsequent analysis of altered expression patterns of the head patterning gene set could be also useful to understand genetic dependencies and phenotypic causalities.

\subsubsection{Tc-foxq2 misregulation leads to neural phenotypes}

foxq2 was already shown to have a neural function in the cnidarian Nematostella and in the deuterostome Strongylocentrotus with an alteration in the neuro-secretory apical organ, in knock-down experiments (Bisgrove and Burke, 1987; Page, 2002; Sinigaglia et al., 2013; Yaguchi et al., 2012, 2010, 2008). A neural foxq2 function was also proposed for protostomes based on the expression pattern (Hunnekuhl and Akam, 2014; Marlow et al., 2014). Here I show that foxq2 has indeed neural functions in protostomes and that $T c$-foxq 2 is involved in brain formation, resulting in defects in the central body and the mushroom bodies upon knock-down and ectopic expression. It appears as if the phenotypes of the knock-down and the gain-of-function experiments were to some degree complementary. In both experiments the antennal lobes remained unaffected, whereas only knock-down experiments showed a fusion of the midline of the two brain hemispheres. In the $T c-f o x q 2$ knock-down phenotype the central body was decreased in size, while in the gain-of-function experiment the central body appeared to be elongated. Further, the medial lobes of the mushroom bodies were fused in knock-down experiments, whereas ectopic Tc-foxq2 expression resulted in an increased spacing between the medial lobes. The neural phenotype in Tc-foxq2 knock-down larvae shared some features with the neural phenotype observed upon Tc-six3 knock-down confirming a mutual regulation of these genes. It has been shown that the neural phenotype correlates with $T c$-six3 function in 
early neuroectodermal patterning events (Posnien et al., 2011b). These findings indicate an early Tc-foxq2 function in neural development as well.

\subsubsection{Outlook}

\subsubsection{Potential extensions of work on $T c$-foxq2 function}

This study could be expanded in the future. New tools of the Tribolium toolbox could be exploited to get a better understanding about $T c$-foxq 2 function and thus a better understanding about genetic and morphogenetic processes happening in the embryonic head.

This work showed that $T c$-foxq2 knock-down results only in a small amount of cuticles showing the strongest phenotype, which is characterized by the loss of the labrum. Thus, the question arises whether this is due to a low penetrance of RNAi treatment or whether this is a compensatory trait of the system.

In order to clarify this issue, one of the first additional experiments, which should be done is the generation of a transgenic Tc-foxq2-null mutant by using the CRISPR/Cas9 system (Gilles et al., 2015; Gilles and Averof, 2014; Hsu et al., 2014; Jinek et al., 2012). This could be achieved by a knock-in of a transformation marker into the coding sequence of $T c$-foxq2. In consequence, this would lead to a loss of $T c$-foxq2 function and would simultaneously provide a marker. These mutants could then be analyzed with respect to their epidermal defects in L1 larvae. It would be interesting whether the penetrance of L1 larvae with a completely absent labrum would be increased and thereby the expressivity of the phenotype strengthened. Further, it should be analyzed whether Tc-foxq2-null mutants show additional defects. Indeed, recent studies in zebrafish showed that phenotypes emanating from transient knock-down experiments (morphants) could be very different from phenotypes resulting from stable transgenic loss-offunction lines (mutants) due to compensatory effects in these mutants (Rossi et al., 2015; Stainier et al., 2015). Hence, a Tc-foxq2-null mutant line could be an opportunity to test whether the epidermal loss-of-function phenotype was complete.

Further, if the mutant shows a more expressive phenotype -including a high penetrance of expressive defects- it would be worth to cross the $T c$-foxq2-null mutant line with the aTub1P-H2Av::EGFP nuclear reporter line. Mutant offspring could be used for in vivo imaging experiments with the LSFM to get new insights into the emergence of the epidermal phenotype. This could give reciprocal new insights into the morphogenesis of the Tribolium head. 
Subsequently, the same should be done using the neural reporter lines to substantiate and refine the findings described in this study (this study, Binzer et al., 2014; Koniszewski et al., 2016; Posnien et al., 2011b).

Exploiting the CRISPR technique it would be also interesting to establish a $T c$-foxq2 enhancer trap line. For this purpose the construct has to be brought into the proximity of the endogenous Tc-foxq2 promoter and under the control of its enhancers. With this line one could perfectly mark $T c$-foxq 2 expressing tissue e.g. the AMR, labrum, and neuroectoderm for in vivo imaging in combination with RNAi to analyze head patterning genes and their effects on embryonic structures. Further it could help to understand head morphogenesis, and it could be used for multiple antibody stainings, when using an anti-EGFP antibody.

\subsubsection{Outlook on neural function}

To track down a potential neural function of $T c-f o x q 2$ it would be helpful to gather more information about the Tc-foxq2 wt expression in the brain. To this end, a Tc-foxq2-specific antibody should be generated. This could be used in immunostainings to get more precise insights about the expression pattern. It would be interesting to analyze whether Tc-foxq2 is expressed in the brain at later embryonic and postembryonic stages (>40 h AEL). In addition to the early neuroectodermal patterning function this would indicate a late neural function. Furthermore, to test whether $T c-f o x q 2$ is expressed in neuroblasts it would be interesting to investigate co-localization with the marker for neuroblasts, Tc-asense (Tc-ase, Brand et al., 1993; Wheeler, 2003). To this end the previously published 'ase-Gal4' line (Koniszewski et al., 2016) could be used in combination with a GAL4-specific antibody together with the Tc-foxq2 antibody in immunostainings. These immunostainings could provide exact data about co-localization and number of $T c-f o x q 2$ positive neuroblasts.

To get a better understanding about the neural phenotype in $T c$-foxq 2 knock-down experiments it could be tested whether the phenotype is due to Tc-foxq2 function in early neuroectodermal patterning or in neural cells at later stages. This could be tested via Tc-foxq2 embryonic RNAi at different stages (Posnien et al., 2011b) or via RNAi within the hsCrPVi1A RNAi inhibitor line, which allows temporal control of RNAi inhibition via heat shock application (J. Ulrich, in prep.). Further, it would be worthwhile to perform Tc-asense (Tc-ase) ISH in $T c$-foxq2 $2^{\text {pRNAi }}$ embryos. Tc-ase, marking the neuroblasts, could provide information in Tc-foxq2 knock-down experiments 
whether neuroblasts show an alteration in number or onset of delamination. Another approach to track down whether $T c$-foxq2 has a neural-specific function would be to knock-down $T c$-foxq2 function in the pattern of Tc-ase, by using the ase-Gal4 line driving a hairpin construct. This line allows interfering specifically with the potential neural aspects and not the ectodermal aspects of $T c$-foxq2 function. The resulting offspring could then be analyzed regarding altered number and composition of neuroblasts, as well as regarding altered expression patterns of neuroblastspecific transcription factors. The neurogenic reporter lines (brainy and MB-green line) could be used to analyze the overall brain defects in Tc-ase driven Tc-foxq2 RNAi offspring.

Moreover, the heat shock-based ectopic Tc-foxq2 expression line could be used for further studies. The described neural phenotype resulted from using embryos at an age of 0-24 h AEL. In order to look for a late neural function of $T c-f o x q 2$ it would be interesting to perform the heat shock treatment at later stages and more narrow time frames. This could help to get more prominent or more specific defects, which could be related easier to Tc-foxq2 function in neural development.

To get a more precise view on the neural defects in Tc-foxq2 knock-down larvae, it would be interesting to dissect L1 larval brains and perform immunostainings with several neural markers, like anti-synapsin, anti-5HT (serotonin), anti-periviscerokinin (PVK), and anti-myoinhibitory protein. Immunostaining against these neuromodulators help to specifically mark parts of the mushroom body and the central body (Koniszewski et al., 2016), which allows more detailed analyses of the defects in larval brains. Especially in combination with high resolution LSM imaging and $3 \mathrm{D}$ reconstruction it would be perfect to get an impression of the threedimensionality of the defects (Dreyer, 2010). However, due to the pleiotropic defects of the brain phenotype it will be difficult to assign the observed effects to certain structures unless more specific imaging lines are available.

\subsubsection{Behavioral assays in Tc-foxq2 knock-down post-embryonic individuals}

This study showed that some Tc-foxq2 knock-down embryos are able to reach L1 larval stages and that these larvae are able, even with the described neural defects, to hatch and to move. Based on these observations it would be interesting to conduct simple behavioral tests with knock-down larvae or if possible with adult individuals. Mushroom bodies are involved in olfactory learning and memory (Akalal et al., 2006; Davis, 2011; Heisenberg, 1998) and the central body, as subunit of the central complex, is involved in locomotor activity, courtship, sky compass orientation, and memory amongst others (Homberg, 2008; Pfeiffer and Homberg, 
2014). Thus, I would propose four different simple behavioral tests: (1) the open field test (Gould et al., 2009; Tremmel and Muller, 2013), (2) light-dark exploration tests (Bourin and Hascoët, 2003; Tremmel and Muller, 2013), (3) odorant recognition tests (Loschiavo, 1965), and (4) the food recognition tests (Campbell and Hagstrum, 2002).

(1) The most promising and easiest test to conduct is the open field test. In this test a group of individuals are put in an empty box and their movement is recorded and tracked with a camera from above. With this test different question could be addressed. (I) Do individuals with neural defects show the same complexity in movement, i.e. the same exploratory behavior than wt individuals? (II) Is the locomotor activity changed? (III) Is the courtship behavior altered?

(2) Light-dark exploration tests are easy to conduct and can give information whether the visual sensing is disrupted. Usually Tribolium individuals tend to choose dark places (C. Schmitt-Engel, personal communication). Loss of preference would point into the direction of processing visual inputs.

(3) With odorant recognition tests it could be analyzed whether they are still responding correctly to different attractive or aversive odors. However, some trials to establish this test within Tribolium already failed because it is hard to get statistically robust results (A. Metzger, personal communication).

(4) The food recognition test could be also very interesting and may give more clear and valid data, because with this test several senses are tested simultaneously. However, the results will give only vague insights of the real causality of the erratic behavior, because it could be based on deficiencies in processing visual, olfactory or tactile clues.

\subsubsection{Uncovering the gene regulatory network of the head \& trying to find novel players for head development}

Recent studies showed that there are still unknown players, which are active in the anterior head gene regulatory network (Kittelmann et al., 2013; Siemanowski et al., 2015). However, the candidate gene approach appears to be exhausted (Economou and Telford, 2009; Kittelmann et al., 2013; Posnien et al., 2011b; Schinko et al., 2008; Schmitt-Engel et al., 2015). The Tribolium iBeetle screen (Schmitt-Engel 2015) is a powerful approach to find novel players acting in the anterior head, even more when the screen achieves the genome-wide level. To this end the 
iBeetle database (Dönitz et al., 2015) can be scanned for annotated labrum specific phenotypes and subsequently analyzed regarding their function in the anterior head gene regulatory network.

In order to identify additional $T c$-foxq2 target genes and to uncover all genetic interactions of the gene regulatory network of the anterior head region, it would be worth exploiting transcriptomic approaches in parallel. A promising approach in Tribolium was recently published using RNAi together with RNA sequencing (RNAi-seq; Oberhofer et al., 2014). Tc-foxq2 would be an interesting candidate gene for this approach, due to its upstream position within the gene regulatory network of the anterior head and because of the lack of secondary effects in early head patterning stages upon knock-down. To this end, $T c-f o x q 2^{\text {pRNAi }}$ embryos have to be generated and afterwards the RNA of early embryonic stages (10-16 h AEL) has to be isolated and sequenced. The Tc-foxq2 RNAi-seq results could substantiate and refine the findings of this study, by quantitatively measuring the effects. A potential drawback of the published RNAi-seq screen (Oberhofer et al., 2014), was that whole embryos were used for the RNA sequencing. This could weaken or blur minor changes in expression level of head players. Thus interesting candidates could be overseen. To overcome this issue I propose to enhance the accuracy of this approach by combining it with the laser capture microdissection (Emmert-Buck et al., 1996) or the laser cutting microdissection (Böhm et al., 1997) technique. With this method the procephalic region could be dissected from the rest of the embryo and afterwards used for RNAi-seq on the basis of morphological traits.

Another approach to get information about the direct genetic interactions of $T c$-foxq 2 with the head patterning genes could be the ChIPSeq (chromatin immunoprecipitation combined with DNA sequencing) technique (Johnson et al., 2007). To this end, a Tc-foxq2 antibody has to be generated first. ChIPSeq using a Tc-foxq2 antibody could help to identify the DNA binding sites of the transcription factor $T c-f o x q 2$. Thus, this technique could provide both new interaction partners and information about direct genetic interaction with other head patterning genes.

\subsubsection{Outlook on the evolutionary aspects of foxq2}

It would be interesting to look at foxq2 function in other arthropods and investigate evolutionary differences. For Drosophila it is known to be expressed at the anterior tip including the clypeolabral region, pharyngeal structures and the brain (Lee and Frasch, 2004). Also the spider Parasteatoda tepidariorum shows an expression at the anterior pole, including the labral region 
(M. Schacht, personal communication). This confirms that the highly conserved expression pattern is also reflected in different arthropod species. Parasteatoda shows a complex expression pattern resembling the expression pattern in Tribolium. Although, co-expression data is lacking so far, it appears that Pt-foxq2 and Pt-six3.1/ Pt-six3. 2 are partially co-expressed, similar to the situation in Tribolium (M. Schacht pers. communication, Schomburg et al., 2015). It would be interesting to determine co-expression of foxq2 and six 3 in wt and in six $3 /$ foxq2 knock-down individuals of these species to uncover potential similarities and changes in function. This would help to get deeper insights into the evolution of the gene regulatory network and, hence, the bases for morphological evolution.

\subsection{New tools to study morphogenetic movements}

\subsubsection{The transgenic C3PA-GFP photoactivation lines are a powerful tool for cell marking and fate mapping}

I was able to generate the first stable transgenic photoactivation lines in Tribolium. These lines are well-suited for marking cells in a targeted manner and for subsequent cell tracing and tracking. However, a potential drawback of this principle could be that the activated signal is reduced over time and with each imaging cycle. This disadvantage hampers imaging over a long time with many imaging intervals. Both lines ( $\alpha$ Tub1P-C3PA-GFP and $\alpha$ Tub1P-H2Av::C3PA-GFP), described in this study, show a strong fluorescent signal after activation using the conventional LSM in combination with UV-light $(350-360 \mathrm{~nm})$. However, the first proof of principle experiments revealed some potential obstacles. Photoactivation with the described settings (see section 3.12) could result in cell cycle arrest, cell death, or changed signal localizations (own observations, A. Gilles personal communication). These observed side-effects are most likely due to phototoxic effects evoked by irradiation with high intensities of UV-light. Since decades it is known that UV-light leads to pyrimidine dimerization, DNA-protein cross-links, and DNA strand break (Kornhauser, 1980; Marrot and Meunier, 2008). These effects interfere with DNA replication, mitosis, and DNA transcription, which eventually result in cytotoxicity, mutagenicity, and the induction of apoptosis leading to cell death and absorption (Marrot and Meunier, 2008; Post et al., 2005). The described effects would explain the observed cell cycle arrest and cell death. Absence of the nuclear signal when activating the H2Av::C3PA-GFP via UV-light could be 
due to cross-linking of these molecules with the DNA, which inhibits the correct folding of the protein, thus resulting in the inability to emit a fluorescent signal. To avoid these side-effects caused by photoactivation, it should first be tested whether the settings of activation could be optimized. To this end, it should be tried to lower the laser intensity or exposure time of the photoactivation. Another possibility could be to use different laser-lines or microscope techniques. First, it could be tested to use a conventional LSM with a $405 \mathrm{~nm}$ laser-line (instead of UV light at $351 \mathrm{~nm}$ and $364 \mathrm{~nm}$ ) for photoactivation, which was already successfully used in Drosophila (Mavrakis et al., 2009). Wavelengths about $400 \mathrm{~nm}$ have less energy and thereby show massively lower rates of side-effects like pyrimidine dimerization and freeing of cytotoxic reactive oxygen species (Marrot and Meunier, 2008). Second, instead of using a conventional LSM, a two-photon microscope could be used. Photoactivation with a two-photon microscope, using light in the infra-red spectrum (780-840 $\mathrm{nm})$, is shown to be efficient and non-toxic et al., 2005; Ruta et al., 2010). If possible, it would be best suited to use a wavelength of $820 \mathrm{~nm}$ for photoactivation for whole mounted embryos (Post et al., 2005).

Another approach could be to use photoactivatable fluorescent proteins, which are activated at a different wavelength. One promising and versatile candidate would be the kindling fluorescent protein-1 (KFP1) based on the chromotprotein asulCP extracted from the sea anemone Anemonia sulcata (Chudakov et al., 2005; Lukyanov et al., 2005). KFP1 shows a red fluorescent signal $(572 / 595 \mathrm{~nm})$ upon photoactivation with intense green light $(590 \mathrm{~nm})$. This signal could be reversibly or irreversibly photoactivated, depending on the activation intensity and duration. Photoactivation could be reversed by using blue light $(450 \mathrm{~nm})$. One advantage of this versatile protein is that e.g. at first the overall morphology can be visualized via a first activation cycle. Afterwards, when the ROI is selected the overall signal could be switched off and only single cells or cell groups within the ROI could be then reactivated. Thus, KFP1 is perfectly-suited for celltracking experiments.

\subsubsection{The genetic Cre/loxP cell marking system is a powerful tool,}

\section{but has to be improved}

I was able to generate a stable transgenic loxP responder line, which could be used, upon crossing with adequate Cre driver lines to mark cells genetically. In this study the loxP responder line was crossed to a heat shock-inducible Cre line, leading to random marking of cells upon heat shock treatment. The loxP responder line, described in this study, is functional regarding the 
fluorescent body marker (mCherry) to visualize embryonic morphology and also regarding the marking of a subset of cells with a different fluorescent marker protein (EGFP). However, the IoxP responder line and the used heat shock-inducible driver line have two problems. First, the fluorescent signal of the mCherry body-marker is weak. This could be explained by the lack of a Kozak consensus sequence (Kozak, 1984) in front of the start codon. The Kozak sequence is involved in ribosomal binding and thereby important for translation initiation. An altered or missing Kozak consensus sequence could decrease translation efficiency and thereby the protein level (Kozak, 1986; Nakagawa et al., 2007). Second, the number of marked cells was far too high for cell tracking experiments both in heat shock-treated as well as in untreated embryos. This suggests some constitutive expression of the Cre recombinase, which is most likely due to the leakiness of the $h s p$ promoter at the integration locus of the $\mathrm{pB}$ [hsp68-Cre] construct. This problem could be overcome by remobilization of the integrated $\mathrm{pB}$ [hsp68-Cre] construct into a less active locus. To achieve this result the Cre driver line has to be crossed to a line that expresses the piggyBac transposase and, which enables the jump of the construct into another locus (Trauner et al., 2009). Afterwards, the resulting offspring has to be tested regarding the green signal indicating the number of marked cells. This should be done in heat shock-treated and in untreated offspring individuals. Offspring that shows no signal when untreated, but an intermediate or strong signal after heat shock treatment should be raised to a larger population and studied in detail.

\subsubsection{The $\alpha$ Tub1/rps3/PUb promoters are ubiquitously active at all}

\section{embryonic stages}

I was able to generate different functional transgenic ubiquitous nuclear reporter lines by synthesizing an H2Av::EGFP chimeric reporter protein that was driven either by the Tc- $\alpha$ Tub1, the Tc-rps3, or the Tc-Pub promoter. However, all these lines showed a strongly decreased viability or led even to an extinction of the complete population after a few generations, as described for the Tc-PUbP-H2Av::EGFP line. The reason for this decreased viability is probably not due to an interference with the overall chromatin structure and cell cycle progression, caused by the $27 \mathrm{kDa}$ EGFP tag (Kanda et al., 1998). It is more likely that the oversupply of the histone $\mathrm{H} 2 \mathrm{~A}$ variant could be the reason for the decreased viability. The transgenic lines carried on the one hand a second copy of this gene and on the other hand this was additionally driven by a highly active promoter. It was already described in several publications that the level of 
histones is crucial for normal development and that excessive histone amounts could lead to RNAi effects, replication errors, altered gene expression, chromosome loss, and death (Groth et al., 2007; Gunjan et al., 2006; Li et al., 2014; Singh et al., 2010). Possible solutions to overcome this problem could be implemented by the use of the CRISPR/Cas9 system (Literature see above). The system could be used to directly fuse a fluorescent protein to the endogenous histone, which is then under the control of the respective histone promoter. Another possibility could be to perform a knock-out of a particular histone and to bring in a histone::fluorescent protein cassette into the first intron of a strongly expressed gene. Thus, the reporter protein will be under control of the desired endogenous promoter.

The newly generated ubiquitous nuclear reporter lines indeed show ubiquitous expression. However, the comparison with the EFA-nGFP (El-Sherif et al., 2012) showed that the expression level is higher only at some developmental stages than in the EFA-nGFP line. At most embryonic stages the EFA-nGFP line showed the best results with respect to signal intensity. However, the new lines and the EFA-nGFP line are not completely comparable due to different reporter proteins. Thus, the search for a good ubiquitous promoter, that drives strong expression, should be continued. The Tc-PUbP was already tested and it showed a strong signal in embryonic stages, but it showed an expression pattern with gaps like the EFA (A. Gilles, personal communication). Another promoter, which could be tested is the Tc-glyceraldehyde-3phosphate dehydrogenase (TC006170, Tcas_OGS 3.0) promoter, a housekeeping gene, which is active in glycolysis. The Tc-actin5C (TCO06296, Tcas_OGS 3.0) promoter could also be an interesting candidate, which is involved in cytoskeletal protein formation. Both show strong expression at embryonic stages of Drosophila (Fisher et al., 2012).

\subsubsection{Utilization of the new imaging lines}

The newly generated imaging lines serve as an expansion for the already versatile Tribolium toolbox. These lines could be used to gain deeper insights into the morphogenesis of the Tribolium head and the complete embryo. These insights could be used to get a better understanding on the cellular processes and tissue movements, which build the final head. Better knowledge of these processes in wt and developmental phenotypes would help to get more precise and detailed information about gene function in morphogenetic processes. 
The aTubP1-H2Av::EGFP line could be used to get more insights on large tissue movements during embryogenesis. By following the established protocols (Strobl et al., 2015) the LSFM technique could be exploited to image the embryo from different angles and hence generate a comprehensive data set (Goldberg et al., 2005). This data set could be processed (Strobl et al., 2015) to finally generate a 4D embryo. Using the Fiji implemented BigDataViewer plug-in (Schindelin et al., 2012) the embryo can be visualized and analyzed from any desired view. Even more, the cell movements could be annotated and tracked in a 4D environment, by using the MaMuT plug-in, which is a combination of the BigDataViewer and TrackMate (Schindelin et al., 2012). However, cell tracking using the ubiquitous nuclear reporter lines is impeded by the fact that the nuclei are tightly packed, permanently in motion and at times covered by the extraembryonic tissue. Thus, automated cell tracking using these lines is impossible and manual cell tracking is at least very difficult and time-consuming. Nevertheless, the combination of the 4D data set and different Fiji plug-ins could be well-suited to test features of the bend \& zipper model (Posnien et al., 2010) or to set up new hypotheses.

The newly established transgenic cell marker line could overcome the drawback of the ubiquitous nuclear reporter lines. These lines could be used to either mark transiently and targeted or permanently and randomly a subset of cells. Marking only small cell populations or single cells facilitates manual cell tracking and could be the first step into the direction of automated cell tracking. This could provide new insights in cell movement and organization, which are needed to set up tissues and to finally build up the larval head. However, this approach is not limited to the head capsule. With these cell marking lines it would be possible to get new insights into the generation of segments from the posterior growth zone. Due to the fact that Drosophila develops as a long-germ embryo, there is no comprehensive data in any insect about embryonic growth involving a posterior growth zone (Benton et al., 2013; Nakamoto et al., 2015; Oberhofer et al., 2014; Sarrazin et al., 2012; Schröder et al., 2008). In contrast to Drosophila, Tribolium develops as short-germ insect, in which segments are progressively added from a posterior growth zone (Tautz et al., 1994). The new cell marking lines could be used to analyze cell behavior in the posterior growth zone and how cells, originating from the posterior growth zone, will contribute to form new segments.

With the IoxP responder line cells could be marked genetically and in a stable manner. The advantage of this method is that marked cells could be traced and tracked in short imaging intervals over a long time of development. The drawback of this tool is that, due to the random marking of cells, a lot of embryos have to be imaged in order to trace the desired cell 
population. However, this tool enables to image the cells of interest throughout embryonic and postembryonic development, which allows the generation of an exact, non-invasive fate map at a cellular resolution. The ultimate goal would be to track down the movement and the final destination for every single cell. Imaging of wild-type embryos and embryos with induced developmental defects would allow new insights into morphogenesis and would facilitate and precise to describe developmental defects and to relate these to gene function.

\subsection{The arthropod head problem}

\subsubsection{Tc-foxq2 - Implementations for the arthropod head problem}

$T c-f o x q 2$ is expressed in the anterior head but not expressed segmental reiterated. Hence, $T c$-foxq2 is another marker for the pre-segmental region. Thus, $T c-f o x q 2$ is substantiating that this region is very different from the rest of the posterior head and from the trunk and relying on a very different set of genes, which is required for its patterning.

The notion that this region is not only a "special segment", but indeed a non-segmental region is even more substantiated by evolutionary data. As already mentioned before, the pre-bilaterian sea anemone Nematostella shows a conserved gene set (foxq2, six 3 , and $r x$ ) required for patterning the aboral (presumably homologous to the anterior) pole (Marlow et al., 2013). These data indicate that these genes were involved in patterning the anterior pole of a pre-bilaterian ancestor, which was unsegmented. The conservation of this gene set, throughout the Metazoa, points to a homology of the anteriormost region among bilateria and its non-segmental structure.

\subsubsection{Imaging lines and implementations for the arthropod head}

\section{problem}

One aspect of the arthropod head problem is dealing with the question, which part of the adult head capsule is built by the embryonic anterior pre-segmental region. The $\alpha$ TubP1-H2Av::C3PA- 
GFP and the $\alpha$ TubP1-C3PA-GFP cell marking lines could be used to answer this question. The transgenic lines allow testing for the contribution of the embryonic anterior pre-segmental region to the larval head capsule as well as the exact contribution of the other segments. To this end, an embryo at a stage where the limbs start to grow out could be mounted. This stage would be well-suited, because the antenna, labral buds and the stomodeum become visible and could be used as morphological landmarks. The anterior pre-segmental region is defined by the expression of marker genes (e.g. Tc-foxq2, Tc-six3, Tc-cnc, Tc-tll, and Tc-orthodentilce1). This defined region could be photoactivated and the marked tissue analyzed regarding its proportion on the L1 larval head. This could shed light onto the acron, i.e. pre-segmental region, contribution to the arthropod head, by revealing its location in larval respectively adult heads. Subsequently, this could be done for every head segment, thereby revealing the contribution to the head capsule in larvae for each head segment. With this fate map it would be easier to understand the origin of head defects, observed in L1 larval cuticle preparations. 
6

\section{References}

Akalal, D.-B.G., Wilson, C.F., Zong, L., Tanaka, N.K., Ito, K., Davis, R.L., 2006. Roles for Drosophila mushroom body neurons in olfactory learning and memory. Learn. Mem. 13, 659-668. doi:10.1101/lm.221206

Akam, M., 1987. The molecular basis for metameric pattern in the Drosophila embryo. Dev. Camb. Engl. 101, 1-22.

Benayoun, B.A., Caburet, S., Veitia, R.A., 2011. Forkhead transcription factors: key players in health and disease. Trends Genet. 27, 224-232. doi:10.1016/j.tig.2011.03.003

Benton, M.A., Akam, M., Pavlopoulos, A., 2013. Cell and tissue dynamics during Tribolium embryogenesis revealed by versatile fluorescence labeling approaches. Development 140, 3210-3220. doi:10.1242/dev.096271

Berghammer, A.J., Klingler, M., Wimmer, E.A., 1999. A universal marker for transgenic insects. Nature 402, 370-371. doi:10.1038/46463

Beutel, R., 2000. Phylogenetic Relationships of the Suborders of Coleoptera (Insecta). Cladistics 16, 103-141. doi:10.1006/clad.1999.0124

Binzer, M., Heuer, C.M., Kollmann, M., Kahnt, J., Hauser, F., Grimmelikhuijzen, C.J.P., Schachtner, J., 2014. Neuropeptidome of Tribolium castaneum antennal lobes and mushroom bodies: Neuropeptides in Tribolium olfactory pathway. J. Comp. Neurol. 522, 337-357. doi:10.1002/cne.23399

Bisgrove, B., Burke, R., 1987. Development of the nervous system of the pluteus larva of Strongylocentrotus droebachiensis. Cell Tissue Res. 248. doi:10.1007/BF00218200

Böhm, M., Wieland, I., Schütze, K., Rübben, H., 1997. Microbeam MOMeNT: non-contact laser microdissection of membrane-mounted native tissue. Am. J. Pathol. 151, 63-67.

Bolker, J., 2012. Model organisms: There's more to life than rats and flies. Nature 491, 31-33. doi:10.1038/491031a

Bolognesi, R., Fischer, T.D., Brown, S.J., 2009. Loss of Tc-arrow and canonical Wnt signaling alters posterior morphology and pair-rule gene expression in the short-germ insect, Tribolium castaneum. Dev. Genes Evol. 219, 369-375. doi:10.1007/s00427-009-0299-3

Bourin, M., Hascoët, M., 2003. The mouse light/dark box test. Eur. J. Pharmacol. 463, 55-65. doi:10.1016/S0014-2999(03)01274-3

Boyan, G.S., Reichert, H., 2011. Mechanisms for complexity in the brain: generating the insect central complex. Trends Neurosci. 34, 247-257. doi:10.1016/j.tins.2011.02.002

Brand, A.H., Manoukian, A.S., Perrimon, N., 1994. Chapter 33 Ectopic Expression in Drosophila, in: Methods in Cell Biology. Elsevier, pp. 635-654.

Brand, M., Jarman, A.P., Jan, L.Y., Jan, Y.N., 1993. asense is a Drosophila neural precursor gene and is capable of initiating sense organ formation. Dev. Camb. Engl. 119, 1-17.

Brönner, G., Chu-LaGraff, Q., Doe, C.Q., Cohen, B., Weigel, D., Taubert, H., Jäckle, H., 1994. $\mathrm{Sp} 1$ /egr-like zinc-finger protein required for endoderm specification and germ-layer formation in Drosophila. Nature 369, 664-668. doi:10.1038/369664a0

Brönner, G., Jäckle, H., 1996. Regulation and function of the terminal gap gene huckebein in the Drosophila blastoderm. Int. J. Dev. Biol. 40, 157-165. 
Bronner, G., Jackle, H., 1991. Control and function of terminal gap gene activity in the posterior pole region of the Drosophila embryo. Mech. Dev. 35, 205-211. doi:10.1016/09254773(91)90019-3

Brown, S., Fellers, J., Shippy, T., Denell, R., Stauber, M., Schmidt-Ott, U., 2001. A strategy for mapping bicoid on the phylogenetic tree. Curr. Biol. 11, R43-R44. doi:10.1016/S09609822(01)00007-0

Brown, S.J., Mahaffey, J.P., Lorenzen, M.D., Denell, R.E., Mahaffey, J.W., 1999. Using RNAi to investigate orthologous homeotic gene function during development of distantly related insects. Evol. Dev. 1, 11-15. doi:10.1046/j.1525-142x.1999.99013.x

Brown, S.J., Patel, N.H., Denell, R.E., 1994. Embryonic expression of the singleTribolium engrailed homolog. Dev. Genet. 15, 7-18. doi:10.1002/dvg.1020150103

Brown, S.J., Shippy, T.D., Miller, S., Bolognesi, R., Beeman, R.W., Lorenzen, M.D., Bucher, G., Wimmer, E.A., Klingler, M., 2009. The Red Flour Beetle, Tribolium castaneum (Coleoptera): A Model for Studies of Development and Pest Biology. Cold Spring Harb. Protoc. 2009, pdb.emo126-emo126. doi:10.1101/pdb.emo126

Bucher, G., Scholten, J., Klingler, M., 2002. Parental RNAi in Tribolium (Coleoptera). Curr. Biol. 12, R85-R86. doi:10.1016/S0960-9822(02)00666-8

Bucher, G., Wimmer, E.A., 2005. Beetle a-head. BIF Futura 20, 164-169.

Budd, G.E., 2002. A palaeontological solution to the arthropod head problem. Nature 417, 271275. doi:10.1038/417271a

Budd, G.E., Telford, M.J., 2009. The origin and evolution of arthropods. Nature 457, 812-817. doi:10.1038/nature07890

Campbell, J.., Hagstrum, D.., 2002. Patch exploitation by Tribolium castaneum: movement patterns, distribution, and oviposition. J. Stored Prod. Res. 38, 55-68. doi:10.1016/S0022-474X(00)00042-4

Carlsson, P., Mahlapuu, M., 2002. Forkhead Transcription Factors: Key Players in Development and Metabolism. Dev. Biol. 250, 1-23. doi:10.1006/dbio.2002.0780

Carroll, S.B., 1995. Homeotic genes and the evolution of arthropods and chordates. Nature 376, 479-485. doi:10.1038/376479a0

Cerny, A.C., Grossmann, D., Bucher, G., Klingler, M., 2008. The Tribolium ortholog of knirps and knirps-related is crucial for head segmentation but plays a minor role during abdominal patterning. Dev. Biol. 321, 284-294. doi:10.1016/j.ydbio.2008.05.527

Chapman, A.D., Australian Biodiversity Information Services, Australian Biological Resources Study, Australia, Department of the Environment, W., Heritage, and the Arts, 2009. Numbers of living species in Australia and the world. Australian Govt., Dept. of the Environment, Water, Heritage, and the Arts, Parkes, ACT.

Chapman, J.A., Kirkness, E.F., Simakov, O., Hampson, S.E., Mitros, T., Weinmaier, T., Rattei, T., Balasubramanian, P.G., Borman, J., Busam, D., Disbennett, K., Pfannkoch, C., Sumin, N., Sutton, G.G., Viswanathan, L.D., Walenz, B., Goodstein, D.M., Hellsten, U., Kawashima, T., Prochnik, S.E., Putnam, N.H., Shu, S., Blumberg, B., Dana, C.E., Gee, L., Kibler, D.F., Law, L., Lindgens, D., Martinez, D.E., Peng, J., Wigge, P.A., Bertulat, B., Guder, C., Nakamura, Y., Ozbek, S., Watanabe, H., Khalturin, K., Hemmrich, G., Franke, A., Augustin, R., Fraune, S., Hayakawa, E., Hayakawa, S., Hirose, M., Hwang, J.S., Ikeo, K., NishimiyaFujisawa, C., Ogura, A., Takahashi, T., Steinmetz, P.R.H., Zhang, X., Aufschnaiter, R., Eder, M.-K., Gorny, A.-K., Salvenmoser, W., Heimberg, A.M., Wheeler, B.M., Peterson, K.J., Böttger, A., Tischler, P., Wolf, A., Gojobori, T., Remington, K.A., Strausberg, R.L., Venter, J.C., Technau, U., Hobmayer, B., Bosch, T.C.G., Holstein, T.W., Fujisawa, T., Bode, H.R., David, C.N., Rokhsar, D.S., Steele, R.E., 2010. The dynamic genome of Hydra. Nature 464, 592-596. doi:10.1038/nature08830

Chapman, R.F., 1982. The insects: structure and function, 3rd ed. ed. Harvard University Press, Cambridge, Mass. 
Chevalier, S., Martin, A., Leclère, L., Amiel, A., Houliston, E., 2006. Polarised expression of FoxB and FoxQ2 genes during development of the hydrozoan Clytia hemisphaerica. Dev. Genes Evol. 216, 709-720. doi:10.1007/s00427-006-0103-6

Chipman, A.D., 2010. Parallel evolution of segmentation by co-option of ancestral gene regulatory networks. BioEssays 32, 60-70. doi:10.1002/bies.200900130

Choe, C.P., Brown, S.J., 2009. Genetic regulation of engrailed and wingless in Tribolium segmentation and the evolution of pair-rule segmentation. Dev. Biol. 325, 482-491. doi:10.1016/j.ydbio.2008.10.037

Choe, C.P., Brown, S.J., 2007. Evolutionary flexibility of pair-rule patterning revealed by functional analysis of secondary pair-rule genes, paired and sloppy-paired in the shortgerm insect, Tribolium castaneum. Dev. Biol. 302, 281-294. doi:10.1016/j.ydbio.2006.09.037

Choe, C.P., Miller, S.C., Brown, S.J., 2006. A pair-rule gene circuit defines segments sequentially in the short-germ insect Tribolium castaneum. Proc. Natl. Acad. Sci. 103, 6560-6564. doi:10.1073/pnas.0510440103

Chudakov, D.M., Lukyanov, S., Lukyanov, K.A., 2005. Fluorescent proteins as a toolkit for in vivo imaging. Trends Biotechnol. 23, 605-613. doi:10.1016/j.tibtech.2005.10.005

Clarkson, M., Saint, R., 1999. A His2AvDGFP Fusion Gene Complements a Lethal His2AvD Mutant Allele and Provides an in Vivo Marker for Drosophila Chromosome Behavior. DNA Cell Biol. 18, 457-462. doi:10.1089/104454999315178

Cohen, S., Jürgens, G., 1991. Drosophila headlines. Trends Genet. 7, 267-272. doi:10.1016/01689525(91)90327-M

Cohen, S.M., Jürgens, G., 1990. Mediation of Drosophila head development by gap-like segmentation genes. Nature 346, 482-485. doi:10.1038/346482a0

Coiffier, D., Charroux, B., Kerridge, S., 2007. Common functions of central and posterior Hox genes for the repression of head in the trunk of Drosophila. Development 135, 291-300. doi:10.1242/dev.009662

Coulcher, J.F., Telford, M.J., 2012. Cap'n'collar differentiates the mandible from the maxilla in the beetle Tribolium castaneum. EvoDevo 3, 25. doi:10.1186/2041-9139-3-25

Crozatier, M., Valle, D., Dubois, L., Ibnsouda, S., Vincent, A., 1999. Head versus trunk patterning in the Drosophila embryo; collier requirement for formation of the intercalary segment. Dev. Camb. Engl. 126, 4385-4394.

Crozatier, M., Valle, D., Dubois, L., Ibnsouda, S., Vincent, A., 1996. collier, a novel regulator of Drosophila head development, is expressed in a single mitotic domain. Curr. Biol. 6, 707718. doi:10.1016/S0960-9822(09)00452-7

Dalton, D., Chadwick, R., McGinnis, W., 1989. Expression and embryonic function of empty spiracles: a Drosophila homeo box gene with two patterning functions on the anteriorposterior axis of the embryo. Genes Dev. 3, 1940-1956. doi:10.1101/gad.3.12a.1940

Darras, S., Gerhart, J., Terasaki, M., Kirschner, M., Lowe, C.J., 2011. -Catenin specifies the endomesoderm and defines the posterior organizer of the hemichordate Saccoglossus kowalevskii. Development 138, 959-970. doi:10.1242/dev.059493

Davis, G.K., Patel, N.H., 2002. S HORT , L ONG , AND B EYOND包: Molecular and Embryological Approaches to Insect Segmentation. Annu. Rev. Entomol. 47, 669-699. doi:10.1146/annurev.ento.47.091201.145251

Davis, R.L., 2011. Traces of Drosophila Memory. Neuron 70, 8-19. doi:10.1016/j.neuron.2011.03.012

de Velasco, B., Erclik, T., Shy, D., Sclafani, J., Lipshitz, H., McInnes, R., Hartenstein, V., 2007. Specification and development of the pars intercerebralis and pars lateralis, neuroendocrine command centers in the Drosophila brain. Dev. Biol. 302, 309-323. doi:10.1016/j.ydbio.2006.09.035 
Dohrmann, M., Worheide, G., 2013. Novel Scenarios of Early Animal Evolution--Is It Time to Rewrite Textbooks? Integr. Comp. Biol. 53, 503-511. doi:10.1093/icb/ict008

Dönitz, J., Grossmann, D., Schild, I., Schmitt-Engel, C., Bradler, S., Prpic, N.-M., Bucher, G., 2013. TrOn: An Anatomical Ontology for the Beetle Tribolium castaneum. PLoS ONE 8, e70695. doi:10.1371/journal.pone.0070695

Dönitz, J., Schmitt-Engel, C., Grossmann, D., Gerischer, L., Tech, M., Schoppmeier, M., Klingler, M., Bucher, G., 2015. iBeetle-Base: a database for RNAi phenotypes in the red flour beetle Tribolium castaneum. Nucleic Acids Res. 43, D720-D725. doi:10.1093/nar/gku1054

Dreyer, 2010. 3D standard brain of the red flour beetle Tribolium castaneum: a tool to study metamorphic development and adult plasticity. Front. Syst. Neurosci. doi:10.3389/neuro.06.003.2010

Economou, A.D., Telford, M.J., 2009. Comparative gene expression in the heads of Drosophila melanogaster and Tribolium castaneum and the segmental affinity of the Drosophila hypopharyngeal lobes. Evol. Dev. 11, 88-96. doi:10.1111/j.1525-142X.2008.00305.x

El-Sherif, E., Averof, M., Brown, S.J., 2012. A segmentation clock operating in blastoderm and germband stages of Tribolium development. Development 139, 4341-4346. doi:10.1242/dev.085126

Emmert-Buck, M.R., Bonner, R.F., Smith, P.D., Chuaqui, R.F., Zhuang, Z., Goldstein, S.R., Weiss, R.A., Liotta, L.A., 1996. Laser Capture Microdissection. Science 274, 998-1001. doi:10.1126/science.274.5289.998

Farzana, L., Brown, S.J., 2008. Hedgehog signaling pathway function conserved in Tribolium segmentation. Dev. Genes Evol. 218, 181-192. doi:10.1007/s00427-008-0207-2

Felsenstein, J., 1985. Confidence Limits on Phylogenies: An Approach Using the Bootstrap. Evolution 39, 783. doi:10.2307/2408678

Finkelstein, R., Smouse, D., Capaci, T.M., Spradling, A.C., Perrimon, N., 1990. The orthodenticle gene encodes a novel homeo domain protein involved in the development of the Drosophila nervous system and ocellar visual structures. Genes Dev. 4, 1516-1527. doi:10.1101/gad.4.9.1516

Fisher, B., Weiszmann, R., Frise, E., Hammonds, A., Tomancak, P., Beaton, A., Berman, B., Quan, E., Shu, S., Lewis, S., Rubin, G., Barale, C., Laguertas, E., Quinn, J., Ghosh, A., Hartenstein, V., Ashburner, M., Celniker, S., 2012. BDGP insitu homepage.

Florentin, A., Arama, E., 2012. Caspase levels and execution efficiencies determine the apoptotic potential of the cell. J. Cell Biol. 196, 513-527. doi:10.1083/jcb.201107133

Fritzenwanker, J.H., Gerhart, J., Freeman, R.M., Lowe, C.J., 2014. The Fox/Forkhead transcription factor family of the hemichordate Saccoglossus kowalevskii. EvoDevo 5, 17. doi:10.1186/2041-9139-5-17

Fu, J., Posnien, N., Bolognesi, R., Fischer, T.D., Rayl, P., Oberhofer, G., Kitzmann, P., Brown, S.J., Bucher, G., 2012. Asymmetrically expressed axin required for anterior development in Tribolium. Proc. Natl. Acad. Sci. 109, 7782-7786. doi:10.1073/pnas.1116641109

Gallitano-Mendel, A., Finkelstein, R., 1998. EctopicorthodenticleExpression Alters Segment Polarity Gene Expression but Not Head Segment Identity in theDrosophilaEmbryo. Dev. Biol. 199, 125-137. doi:10.1006/dbio.1998.8917

Gilles, A.F., Averof, M., 2014. Functional genetics for all: engineered nucleases, CRISPR and the gene editing revolution. EvoDevo 5, 43. doi:10.1186/2041-9139-5-43

Gilles, A.F., Schinko, J.B., Averof, M., 2015. Efficient CRISPR-mediated gene targeting and transgene replacement in the beetle Tribolium castaneum. Development 142, 28322839. doi:10.1242/dev.125054

Goldberg, I.G., Allan, C., Burel, J.-M., Creager, D., Falconi, A., Hochheiser, H., Johnston, J., Mellen, J., Sorger, P.K., Swedlow, J.R., 2005. The Open Microscopy Environment (OME) Data 
Model and XML file: open tools for informatics and quantitative analysis in biological imaging. Genome Biol. 6, R47. doi:10.1186/gb-2005-6-5-r47

Gould, T.D., Dao, D.T., Kovacsics, C.E., 2009. The Open Field Test, in: Gould, T.D. (Ed.), Mood and Anxiety Related Phenotypes in Mice. Humana Press, Totowa, NJ, pp. 1-20.

Grimaldi, D.A., Engel, M.S., 2005. Evolution of the insects. Cambridge University Press, Cambridge [U.K.] ; New York.

Grossniklaus, U., Cadigan, K.M., Gehring, W.J., 1994. Three maternal coordinate systems cooperate in the patterning of the Drosophila head. Dev. Camb. Engl. 120, 3155-3171.

Grossniklaus, U., Pearson, R.K., Gehring, W.J., 1992. The Drosophila sloppy paired locus encodes two proteins involved in segmentation that show homology to mammalian transcription factors. Genes Dev. 6, 1030-1051. doi:10.1101/gad.6.6.1030

Groth, A., Corpet, A., Cook, A.J.L., Roche, D., Bartek, J., Lukas, J., Almouzni, G., 2007. Regulation of Replication Fork Progression Through Histone Supply and Demand. Science 318, 1928-1931. doi:10.1126/science.1148992

Gunjan, A., Paik, J., Verreault, A., 2006. The emergence of regulated histone proteolysis. Curr. Opin. Genet. Dev. 16, 112-118. doi:10.1016/j.gde.2006.02.010

Haas, M.S., Brown, S.J., Beeman, R.W., 2001. Homeotic evidence for the appendicular origin of the labrum in Tribolium castaneum. Dev. Genes Evol. 211, 96-102. doi:10.1007/s004270000129

Häcker, U., Kaufmann, E., Hartmann, C., Jürgens, G., Knöchel, W., Jäckle, H., 1995. The Drosophila fork head domain protein crocodile is required for the establishment of head structures. EMBO J. 14, 5306-5317.

Hannenhalli, S., Kaestner, K.H., 2009. The evolution of Fox genes and their role in development and disease. Nat. Rev. Genet. 10, 233-240. doi:10.1038/nrg2523

Heisenberg, M., 1998. What do the mushroom bodies do for the insect brain? an introduction. Learn. Mem. Cold Spring Harb. N 5, 1-10.

Homberg, U., 2008. Evolution of the central complex in the arthropod brain with respect to the visual system. Arthropod Struct. Dev. 37, 347-362. doi:10.1016/j.asd.2008.01.008

Hope, I., 2003. The forkhead gene family of Caenorhabditis elegans. Gene 304, 43-55. doi:10.1016/S0378-1119(02)01175-7

Howard-Ashby, M., Materna, S.C., Brown, C.T., Chen, L., Cameron, R.A., Davidson, E.H., 2006. Identification and characterization of homeobox transcription factor genes in Strongylocentrotus purpuratus, and their expression in embryonic development. Dev. Biol. 300, 74-89. doi:10.1016/j.ydbio.2006.08.039

Hsu, P.D., Lander, E.S., Zhang, F., 2014. Development and Applications of CRISPR-Cas9 for Genome Engineering. Cell 157, 1262-1278. doi:10.1016/j.cell.2014.05.010

Hunnekuhl, V.S., Akam, M., 2014. An anterior medial cell population with an apical-organ-like transcriptional profile that pioneers the central nervous system in the centipede Strigamia maritima. Dev. Biol. 396, 136-149. doi:10.1016/j.ydbio.2014.09.020

Ingham, P.W., 1988. The molecular genetics of embryonic pattern formation in Drosophila. Nature 335, 25-34. doi:10.1038/335025a0

Izeddin, I., Specht, C.G., Lelek, M., Darzacq, X., Triller, A., Zimmer, C., Dahan, M., 2011. SuperResolution Dynamic Imaging of Dendritic Spines Using a Low-Affinity Photoconvertible Actin Probe. PLoS ONE 6, e15611. doi:10.1371/journal.pone.0015611

Jinek, M., Chylinski, K., Fonfara, I., Hauer, M., Doudna, J.A., Charpentier, E., 2012. A Programmable Dual-RNA-Guided DNA Endonuclease in Adaptive Bacterial Immunity. Science 337, 816-821. doi:10.1126/science.1225829

Johnson, D.S., Mortazavi, A., Myers, R.M., Wold, B., 2007. Genome-Wide Mapping of in Vivo Protein-DNA Interactions. Science 316, 1497-1502. doi:10.1126/science.1141319

Johnston, D.S., Nüsslein-Volhard, C., 1992. The origin of pattern and polarity in the Drosophila embryo. Cell 68, 201-219. doi:10.1016/0092-8674(92)90466-P 
Jürgens, G., Lehmann, R., Schardin, M., Nüsslein-Volhard, C., 1986. Segmental organisation of the head in the embryo of Drosophila melanogaster: A blastoderm fate map of the cuticle structures of the larval head. Rouxs Arch. Dev. Biol. 195, 359-377. doi:10.1007/BF00402870

Kaestner, K.H., Knochel, W., Martinez, D.E., 2000. Unified nomenclature for the winged helix/forkhead transcription factors. Genes Dev. 14, 142-146.

Kanda, T., Sullivan, K.F., Wahl, G.M., 1998. Histone-GFP fusion protein enables sensitive analysis of chromosome dynamics in living mammalian cells. Curr. Biol. 8, 377-385. doi:10.1016/S0960-9822(98)70156-3

Kittelmann, S., 2012. Formation of the Clypeolabral Region During Embryonic Head Development of the Red Flour Beetle Tribolium castaneum. Universität Göttingen.

Kittelmann, S., Ulrich, J., Posnien, N., Bucher, G., 2013. Changes in anterior head patterning underlie the evolution of long germ embryogenesis. Dev. Biol. 374, 174-184. doi:10.1016/j.ydbio.2012.11.026

Kitzmann, P., Schwirz, J., Schmitt-Engel, C., Bucher, G., 2013. RNAi phenotypes are influenced by the genetic background of the injected strain. BMC Genomics 14, 5. doi:10.1186/14712164-14-5

Klingler, M., 2004. Tribolium. Curr. Biol. 14, R639-R640. doi:10.1016/j.cub.2004.08.004

Kohler, R.E., 1994. Lords of the fly: Drosophila genetics and the experimental life. University of Chicago Press, Chicago.

Koniszewski, N., 2011. Functional analysis of embryonic brain development in Tribolium castaneum. Universität Göttingen.

Koniszewski, N.D.B., Kollmann, M., Bigham, M., Farnworth, M., He, B., Büscher, M., Hütteroth, W., Binzer, M., Schachtner, J., Bucher, G., 2016. The insect central complex as model for heterochronic brain development-background, concepts, and tools. Dev. Genes Evol. doi:10.1007/s00427-016-0542-7

Kornhauser, A., 1980. Molecular Aspects of Phototoxicity. Ann. N. Y. Acad. Sci. 346, 398-414. doi:10.1111/j.1749-6632.1980.tb22112.x

Kozak, M., 1986. Point mutations define a sequence flanking the AUG initiator codon that modulates translation by eukaryotic ribosomes. Cell 44, 283-292. doi:10.1016/00928674(86)90762-2

Kozak, M., 1984. Compilation and analysis of sequences upstream from the translational start site in eukaryotic mRNAs. Nucleic Acids Res. 12, 857-872. doi:10.1093/nar/12.2.857

Koziol, U., Jarero, F., Olson, P.D., Brehm, K., 2016. Comparative analysis of Wnt expression identifies a highly conserved developmental transition in flatworms. BMC Biol. 14. doi:10.1186/s12915-016-0233-x

Lai, E., Prezioso, V.R., Smith, E., Litvin, O., Costa, R.H., Darnell, J.E., 1990. HNF-3A, a hepatocyteenriched transcription factor of novel structure is regulated transcriptionally. Genes Dev. 4, 1427-1436. doi:10.1101/gad.4.8.1427

Lam, E.W.-F., Brosens, J.J., Gomes, A.R., Koo, C.-Y., 2013. Forkhead box proteins: tuning forks for transcriptional harmony. Nat. Rev. Cancer 13, 482-495. doi:10.1038/nrc3539

Larroux, C., Luke, G.N., Koopman, P., Rokhsar, D.S., Shimeld, S.M., Degnan, B.M., 2008. Genesis and Expansion of Metazoan Transcription Factor Gene Classes. Mol. Biol. Evol. 25, 980996. doi:10.1093/molbev/msn047

Lee, H.-H., Frasch, M., 2004. Survey of forkhead domain encoding genes in theDrosophila genome: Classification and embryonic expression patterns. Dev. Dyn. 229, 357-366. doi:10.1002/dvdy.10443

Lemons, D., 2006. Genomic Evolution of Hox Gene Clusters. Science 313, 1918-1922. doi:10.1126/science.1132040 
Li, Z., Johnson, M.R., Ke, Z., Chen, L., Welte, M.A., 2014. Drosophila Lipid Droplets Buffer the H2Av Supply to Protect Early Embryonic Development. Curr. Biol. 24, 1485-1491. doi:10.1016/j.cub.2014.05.022

Lindquist, S., 1986. The Heat-Shock Response. Annu. Rev. Biochem. 55, 1151-1191. doi:10.1146/annurev.bi.55.070186.005443

Livet, J., 2007. Brainbow ou le cerveau en couleurs. médecine/sciences 23, 1173-1176. doi:10.1051/medsci/200723121173

Lorenzen, M.D., Berghammer, A.J., Brown, S.J., Denell, R.E., Klingler, M., Beeman, R.W., 2003. piggyBac-mediated germline transformation in the beetle Tribolium castaneum. Insect Mol. Biol. 12, 433-440. doi:10.1046/j.1365-2583.2003.00427.x

Lorenzen, M.D., Brown, S.J., Denell, R.E., Beeman, R.W., 2002. Cloning and characterization of the Tribolium castaneum eye-color genes encoding tryptophan oxygenase and kynurenine 3-monooxygenase. Genetics 160, 225-234.

Loschiavo, S.R., 1965. Methods for Studying Aggregation and Feeding Behavior of the Confused Flour Beetle, Tribolium confusum (Coleoptera: Tenebrionidae). Ann. Entomol. Soc. Am. 58, 383-388. doi:10.1093/aesa/58.3.383

Lukyanov, K.A., Chudakov, D.M., Lukyanov, S., Verkhusha, V.V., 2005. Innovation: Photoactivatable fluorescent proteins. Nat. Rev. Mol. Cell Biol. 6, 885-890. doi:10.1038/nrm1741

Lynch, J., Desplan, C., 2003. "De-evolution" of Drosophila toward a more generic mode of axis patterning. Int. J. Dev. Biol. 47, 497-503.

Marlow, H., Matus, D.Q., Martindale, M.Q., 2013. Ectopic activation of the canonical wnt signaling pathway affects ectodermal patterning along the primary axis during larval development in the anthozoan Nematostella vectensis. Dev. Biol. 380, 324-334. doi:10.1016/j.ydbio.2013.05.022

Marlow, H., Tosches, M.A., Tomer, R., Steinmetz, P.R., Lauri, A., Larsson, T., Arendt, D., 2014. Larval body patterning and apical organs are conserved in animal evolution. BMC Biol. 12, 7. doi:10.1186/1741-7007-12-7

Marrot, L., Meunier, J.-R., 2008. Skin DNA photodamage and its biological consequences. J. Am. Acad. Dermatol. 58, S139-S148. doi:10.1016/j.jaad.2007.12.007

Martín-Durán, J.M., Hejnol, A., 2015. The study of Priapulus caudatus reveals conserved molecular patterning underlying different gut morphogenesis in the Ecdysozoa. BMC Biol. 13. doi:10.1186/s12915-015-0139-z

Martín-Durán, J.M., Vellutini, B.C., Hejnol, A., 2015. Evolution and development of the adelphophagic, intracapsular Schmidt's larva of the nemertean Lineus ruber. EvoDevo 6. doi:10.1186/s13227-015-0023-5

Mavrakis, M., Rikhy, R., Lippincott-Schwartz, J., 2009. Plasma Membrane Polarity and Compartmentalization Are Established before Cellularization in the Fly Embryo. Dev. Cell 16, 93-104. doi:10.1016/j.devcel.2008.11.003

Mazet, F., Yu, J.-K., Liberles, D.A., Holland, L.Z., Shimeld, S.M., 2003. Phylogenetic relationships of the Fox (Forkhead) gene family in the Bilateria. Gene 316, 79-89. doi:10.1016/S03781119(03)00741-8

Merrill, V.K.L., Diederich, R.J., Turner, F.R., Kaufman, T.C., 1989. A genetic and developmental analysis of mutations in labial, a gene necessary for proper head formation in Drosophila melanogaster. Dev. Biol. 135, 376-391. doi:10.1016/0012-1606(89)90187-5

Metzger, D., 1999. Engineering the mouse genome by site-specific recombination. Curr. Opin. Biotechnol. 10, 470-476. doi:10.1016/S0958-1669(99)00012-9

Mohler, J., 1995. Spatial regulation of segment polarity gene expression in the anterior terminal region of the Drosophila blastoderm embryo. Mech. Dev. 50, 151-161.

doi:10.1016/0925-4773(94)00332-H 
Mohler, J., 1993. Genetic regulation of CNC expression in the pharnygeal primordia ofDrosophila blastoderm embryos. Rouxs Arch. Dev. Biol. 202, 214-223. doi:10.1007/BF02427882

Nagy, L.M., Carroll, S., 1994. Conservation of wingless patterning functions in the short-germ embryos of Tribolium castaneum. Nature 367, 460-463. doi:10.1038/367460a0

Nakagawa, S., Niimura, Y., Gojobori, T., Tanaka, H., Miura, K. -i., 2007. Diversity of preferred nucleotide sequences around the translation initiation codon in eukaryote genomes. Nucleic Acids Res. 36, 861-871. doi:10.1093/nar/gkm1102

Nakamoto, A., Hester, S.D., Constantinou, S.J., Blaine, W.G., Tewksbury, A.B., Matei, M.T., Nagy, L.M., Williams, T.A., 2015. Changing cell behaviours during beetle embryogenesis correlates with slowing of segmentation. Nat. Commun. 6, 6635. doi:10.1038/ncomms7635

Oberhofer, G., Grossmann, D., Siemanowski, J.L., Beissbarth, T., Bucher, G., 2014. Wnt/ -catenin signaling integrates patterning and metabolism of the insect growth zone. Development 141, 4740-4750. doi:10.1242/dev.112797

Oppenheimer, D.I., MacNicol, A.M., Patel, N.H., 1999. Functional conservation of the winglessengrailed interaction as shown by a widely applicable baculovirus misexpression system. Curr. Biol. 9, 1288-1296. doi:10.1016/S0960-9822(00)80050-0

Ou, Q., Shu, D., Mayer, G., 2012. Cambrian lobopodians and extant onychophorans provide new insights into early cephalization in Panarthropoda. Nat. Commun. 3, 1261. doi:10.1038/ncomms2272

Page, L.R., 2002. Larval and metamorphic development of the foregut and proboscis in the caenogastropodMarsenina (Lamellaria) stearnsii. J. Morphol. 252, 202-217. doi:10.1002/jmor.1099

Patterson, G.H., 2002. A Photoactivatable GFP for Selective Photolabeling of Proteins and Cells. Science 297, 1873-1877. doi:10.1126/science.1074952

Pauls, S., Geldmacher-Voss, B., Campos-Ortega, J.A., 2001. A zebrafish histone variant H2A.F/Z and a transgenic H2A.F/Z:GFP fusion protein for in vivo studies of embryonic development. Dev. Genes Evol. 211, 603-610. doi:10.1007/s00427-001-0196-x

Pearson, J.C., Lemons, D., McGinnis, W., 2005. Modulating Hox gene functions during animal body patterning. Nat. Rev. Genet. 6, 893-904. doi:10.1038/nrg1726

Peel, A.D., Schanda, J., Grossmann, D., Ruge, F., Oberhofer, G., Gilles, A.F., Schinko, J.B., Klingler, M., Bucher, G., 2013. Tc-knirps plays different roles in the specification of antennal and mandibular parasegment boundaries and is regulated by a pair-rule gene in the beetle Tribolium castaneum. BMC Dev. Biol. 13, 25. doi:10.1186/1471-213X-13-25

Pfeiffer, K., Homberg, U., 2014. Organization and Functional Roles of the Central Complex in the Insect Brain. Annu. Rev. Entomol. 59, 165-184. doi:10.1146/annurev-ento-011613162031

Pick, L., 1998. Segmentation: Painting stripes from flies to vertebrates. Dev. Genet. 23, 1-10. doi:10.1002/(SICI)1520-6408(1998)23:1<1::AID-DVG1>3.0.CO;2-A

Posnien, N., Bashasab, F., Bucher, G., 2009. The insect upper lip (labrum) is a nonsegmental appendage-like structure. Evol. Dev. 11, 480-488. doi:10.1111/j.1525142X.2009.00356.X

Posnien, N., Bucher, G., 2010. Formation of the insect head involves lateral contribution of the intercalary segment, which depends on Tc-labial function. Dev. Biol. 338, 107-116. doi:10.1016/j.ydbio.2009.11.010

Posnien, N., Koniszewski, N., Bucher, G., 2011a. Insect Tc-six4 marks a unit with similarity to vertebrate placodes. Dev. Biol. 350, 208-216. doi:10.1016/j.ydbio.2010.10.024

Posnien, N., Koniszewski, N.D.B., Hein, H.J., Bucher, G., 2011b. Candidate Gene Screen in the Red Flour Beetle Tribolium Reveals Six3 as Ancient Regulator of Anterior Median Head and Central Complex Development. PLoS Genet. 7, e1002416.

doi:10.1371/journal.pgen.1002416 
Posnien, N., Schinko, J., Grossmann, D., Shippy, T.D., Konopova, B., Bucher, G., 2009. RNAi in the Red Flour Beetle (Tribolium). Cold Spring Harb. Protoc. 2009, pdb.prot5256-prot5256. doi:10.1101/pdb.prot5256

Posnien, N., Schinko, J.B., Kittelmann, S., Bucher, G., 2010. Genetics, development and composition of the insect head - A beetle's view. Arthropod Struct. Dev. 39, 399-410. doi:10.1016/j.asd.2010.08.002

Post, J.N., Lidke, K.A., Rieger, B., Arndt-Jovin, D.J., 2005. One- and two-photon photoactivation of a paGFP-fusion protein in live Drosophila embryos. FEBS Lett. 579, 325-330. doi:10.1016/j.febslet.2004.11.092

Prelich, G., 2012. Gene Overexpression: Uses, Mechanisms, and Interpretation. Genetics 190, 841-854. doi:10.1534/genetics.111.136911

Prud'homme, B., de Rosa, R., Arendt, D., Julien, J.-F., Pajaziti, R., Dorresteijn, A.W.C., Adoutte, A., Wittbrodt, J., Balavoine, G., 2003. Arthropod-like Expression Patterns of engrailed and wingless in the Annelid Platynereis dumerilii Suggest a Role in Segment Formation. Curr. Biol. 13, 1876-1881. doi:10.1016/j.cub.2003.10.006

Qiu, S., 2005. A computational study of off-target effects of RNA interference. Nucleic Acids Res. 33, 1834-1847. doi:10.1093/nar/gki324

Range, R.C., Wei, Z., 2016. An anterior signaling center patterns and sizes the anterior neuroectoderm of the sea urchin embryo. Development. doi:10.1242/dev.128165

Rempel, J.G., 1975. The evolution of the insect head: the endless dispute. Quaest. Entomol. 725.

Reuter, R., Leptin, M., 1994. Interacting functions of snail, twist and huckebein during the early development of germ layers in Drosophila. Dev. Camb. Engl. 120, 1137-1150.

Richards, S., Gibbs, R.A., Weinstock, G.M., Brown, S.J., Denell, R., Beeman, R.W., Gibbs, R., Beeman, R.W., Brown, S.J., Bucher, G., Friedrich, M., Grimmelikhuijzen, C.J.P., Klingler, M., Lorenzen, M., Richards, S., Roth, S., Schröder, R., Tautz, D., Zdobnov, E.M., Muzny, D., Gibbs, R.A., Weinstock, G.M., Attaway, T., Bell, S., Buhay, C.J., Chandrabose, M.N., Chavez, D., Clerk-Blankenburg, K.P., Cree, A., Dao, M., Davis, C., Chacko, J., Dinh, H., Dugan-Rocha, S., Fowler, G., Garner, T.T., Garnes, J., Gnirke, A., Hawes, A., Hernandez, J., Hines, S., Holder, M., Hume, J., Jhangiani, S.N., Joshi, V., Khan, Z.M., Jackson, L., Kovar, C., Kowis, A., Lee, S., Lewis, L.R., Margolis, J., Morgan, M., Nazareth, L.V., Nguyen, N., Okwuonu, G., Parker, D., Richards, S., Ruiz, S.-J., Santibanez, J., Savard, J., Scherer, S.E., Schneider, B., Sodergren, E., Tautz, D., Vattahil, S., Villasana, D., White, C.S., Wright, R., Park, Y., Beeman, R.W., Lord, J., Oppert, B., Lorenzen, M., Brown, S., Wang, L., Savard, J., Tautz, D., Richards, S., Weinstock, G., Gibbs, R.A., Liu, Y., Worley, K., Weinstock, G., Elsik, C.G., Reese, J.T., Elhaik, E., Landan, G., Graur, D., Arensburger, P., Atkinson, P., Beeman, R.W., Beidler, J., Brown, S.J., Demuth, J.P., Drury, D.W., Du, Y.-Z., Fujiwara, H., Lorenzen, M., Maselli, V., Osanai, M., Park, Y., Robertson, H.M., Tu, Z., Wang, J., Wang, S., Richards, S., Song, H., Zhang, L., Sodergren, E., Werner, D., Stanke, M., Morgenstern, B., Solovyev, V., Kosarev, P., Brown, G., Chen, H.-C., Ermolaeva, O., Hlavina, W., Kapustin, Y., Kiryutin, B., Kitts, P., Maglott, D., Pruitt, K., Sapojnikov, V., Souvorov, A., Mackey, A.J., Waterhouse, R.M., Wyder, S., Zdobnov, E.M., Zdobnov, E.M., Wyder, S., Kriventseva, E.V., Kadowaki, T., Bork, P., Aranda, M., Bao, R., Beermann, A., Berns, N., Bolognesi, R., Bonneton, F., Bopp, D., Brown, S.J., Bucher, G., Butts, T., Chaumot, A., Denell, R.E., Ferrier, D.E.K., Friedrich, M., Gordon, C.M., Jindra, M., Klingler, M., Lan, Q., Lattorff, H.M.G., Laudet, V., von Levetsow, C., Liu, Z., Lutz, R., Lynch, J.A., da Fonseca, R.N., Posnien, N., Reuter, R., Roth, S., Savard, J., Schinko, J.B., Schmitt, C., Schoppmeier, M., Schröder, R., Shippy, T.D., Simonnet, F., Marques-Souza, H., Tautz, D., Tomoyasu, Y., Trauner, J., Van der Zee, M., Vervoort, M., Wittkopp, N., Wimmer, E.A., Yang, X., Jones, A.K., Sattelle, D.B., Ebert, P.R., Nelson, D., Scott, J.G., Beeman, R.W., Muthukrishnan, S., Kramer, K.J., Arakane, Y., Beeman, R.W., Zhu, Q., Hogenkamp, D., Dixit, R., Oppert, B., 
Jiang, H., Zou, Z., Marshall, J., Elpidina, E., Vinokurov, K., Oppert, C., Zou, Z., Evans, J., Lu, Z., Zhao, P., Sumathipala, N., Altincicek, B., Vilcinskas, A., Williams, M., Hultmark, D., Hetru, C., Jiang, H., Grimmelikhuijzen, C.J.P., Hauser, F., Cazzamali, G., Williamson, M., Park, Y., Li, B., Tanaka, Y., Predel, R., Neupert, S., Schachtner, J., Verleyen, P., Raible, F., Bork, P., Friedrich, M., Walden, K.K.O., Robertson, H.M., Angeli, S., Forêt, S., Bucher, G., Schuetz, S., Maleszka, R., Wimmer, E.A., Beeman, R.W., Lorenzen, M., Tomoyasu, Y., Miller, S.C., Grossmann, D., Bucher, G., 2008. The genome of the model beetle and pest Tribolium castaneum. Nature 452, 949-955. doi:10.1038/nature06784

Rogers, B.T., Kaufman, T.C., 1997. Structure of the Insect Head in Ontogeny and Phylogeny: A View from Drosophila, in: International Review of Cytology. Elsevier, pp. 1-84.

Rossi, A., Kontarakis, Z., Gerri, C., Nolte, H., Hölper, S., Krüger, M., Stainier, D.Y.R., 2015. Genetic compensation induced by deleterious mutations but not gene knockdowns. Nature 524, 230-233. doi:10.1038/nature14580

Ruta, V., Datta, S.R., Vasconcelos, M.L., Freeland, J., Looger, L.L., Axel, R., 2010. A dimorphic pheromone circuit in Drosophila from sensory input to descending output. Nature 468, 686-690. doi:10.1038/nature09554

Sander, K., 1976. Specification of the Basic Body Pattern in Insect Embryogenesis, in: Advances in Insect Physiology. Elsevier, pp. 125-238.

Santagata, S., Resh, C., Hejnol, A., Martindale, M.Q., Passamaneck, Y.J., 2012. Development of the larval anterior neurogenic domains of Terebratalia transversa (Brachiopoda) provides insights into the diversification of larval apical organs and the spiralian nervous system. EvoDevo 3, 3. doi:10.1186/2041-9139-3-3

Sarrazin, A.F., Peel, A.D., Averof, M., 2012. A Segmentation Clock with Two-Segment Periodicity in Insects. Science 336, 338-341. doi:10.1126/science.1218256

Schaeper, N.D., Pechmann, M., Damen, W.G.M., Prpic, N.-M., Wimmer, E.A., 2010. Evolutionary plasticity of collier function in head development of diverse arthropods. Dev. Biol. 344, 363-376. doi:10.1016/j.ydbio.2010.05.001

Schindelin, J., Arganda-Carreras, I., Frise, E., Kaynig, V., Longair, M., Pietzsch, T., Preibisch, S., Rueden, C., Saalfeld, S., Schmid, B., Tinevez, J.-Y., White, D.J., Hartenstein, V., Eliceiri, K., Tomancak, P., Cardona, A., 2012. Fiji: an open-source platform for biological-image analysis. Nat. Methods 9, 676-682. doi:10.1038/nmeth.2019

Schinko, J., Posnien, N., Kittelmann, S., Koniszewski, N., Bucher, G., 2009. Single and Double Whole-Mount In Situ Hybridization in Red Flour Beetle (Tribolium) Embryos. Cold Spring Harb. Protoc. 2009, pdb.prot5258-prot5258. doi:10.1101/pdb.prot5258

Schinko, J.B., Hillebrand, K., Bucher, G., 2012. Heat shock-mediated misexpression of genes in the beetle Tribolium castaneum. Dev. Genes Evol. 222, 287-298. doi:10.1007/s00427012-0412-x

Schinko, J.B., Kreuzer, N., Offen, N., Posnien, N., Wimmer, E.A., Bucher, G., 2008. Divergent functions of orthodenticle, empty spiracles and buttonhead in early head patterning of the beetle Tribolium castaneum (Coleoptera). Dev. Biol. 317, 600-613. doi:10.1016/j.ydbio.2008.03.005

Schinko, J.B., Weber, M., Viktorinova, I., Kiupakis, A., Averof, M., Klingler, M., Wimmer, E.A., Bucher, G., 2010. Functionality of the GAL4/UAS system in Tribolium requires the use of endogenous core promoters. BMC Dev. Biol. 10, 53. doi:10.1186/1471-213X-10-53

Schmidt-Ott, U., González-Gaitán, M., Jäckle, H., Technau, G.M., 1994. Number, identity, and sequence of the Drosophila head segments as revealed by neural elements and their deletion patterns in mutants. Proc. Natl. Acad. Sci. U. S. A. 91, 8363-8367.

Schmidt-Ott, U., Technau, G.M., 1992. Expression of en and wg in the embryonic head and brain of Drosophila indicates a refolded band of seven segment remnants. Dev. Camb. Engl. 116, 111-125. 
Schmitt-Engel, C., Schultheis, D., Schwirz, J., Ströhlein, N., Troelenberg, N., Majumdar, U., Dao, V.A., Grossmann, D., Richter, T., Tech, M., Dönitz, J., Gerischer, L., Theis, M., Schild, I., Trauner, J., Koniszewski, N.D.B., Küster, E., Kittelmann, S., Hu, Y., Lehmann, S., Siemanowski, J., Ulrich, J., Panfilio, K.A., Schröder, R., Morgenstern, B., Stanke, M., Buchhholz, F., Frasch, M., Roth, S., Wimmer, E.A., Schoppmeier, M., Klingler, M., Bucher, G., 2015. The iBeetle large-scale RNAi screen reveals gene functions for insect development and physiology. Nat. Commun. 6, 7822. doi:10.1038/ncomms8822

Scholtz, G., Edgecombe, G.D., 2006. The evolution of arthropod heads: reconciling morphological, developmental and palaeontological evidence. Dev. Genes Evol. 216, 395-415. doi:10.1007/s00427-006-0085-4

Schomburg, C., Turetzek, N., Schacht, M.I., Schneider, J., Kirfel, P., Prpic, N.-M., Posnien, N., 2015. Molecular characterization and embryonic origin of the eyes in the common house spider Parasteatoda tepidariorum. EvoDevo 6. doi:10.1186/s13227-015-0011-9

Schoppmeier, M., Fischer, S., Schmitt-Engel, C., Löhr, U., Klingler, M., 2009. An Ancient Anterior Patterning System Promotes Caudal Repression and Head Formation in Ecdysozoa. Curr. Biol. 19, 1811-1815. doi:10.1016/j.cub.2009.09.026

Schoppmeier, M., Schröder, R., 2005. Maternal Torso Signaling Controls Body Axis Elongation in a Short Germ Insect. Curr. Biol. 15, 2131-2136. doi:10.1016/j.cub.2005.10.036

Schröder, R., Beermann, A., Wittkopp, N., Lutz, R., 2008. From development to biodiversityTribolium castaneum, an insect model organism for short germband development. Dev. Genes Evol. 218, 119-126. doi:10.1007/s00427-008-0214-3

Schwartz, R.M., Dayhoff, M.O., 1979. Protein and nucleic Acid sequence data and phylogeny. Science 205, 1038-1039. doi:10.1126/science.205.4410.1038

Shimeld, S.M., Degnan, B., Luke, G.N., 2010. Evolutionary genomics of the Fox genes: Origin of gene families and the ancestry of gene clusters. Genomics 95, 256-260. doi:10.1016/j.ygeno.2009.08.002

Siebert, K.S., Lorenzen, M.D., Brown, S.J., Park, Y., Beeman, R.W., 2008. Tubulin superfamily genes in Tribolium castaneum and the use of a Tubulin promoter to drive transgene expression. Insect Biochem. Mol. Biol. 38, 749-755. doi:10.1016/j.ibmb.2008.04.007

Siemanowski, J., Richter, T., Dao, V.A., Bucher, G., 2015. Notch signaling induces cell proliferation in the labrum in a regulatory network different from the thoracic legs. Dev. Biol. 408, 164-177. doi:10.1016/j.ydbio.2015.09.018

Singh, R.K., Liang, D., Gajjalaiahvari, U.R., Kabbaj, M.-H.M., Paik, J., Gunjan, A., 2010. Excess histone levels mediate cytotoxicity via multiple mechanisms. Cell Cycle 9, 4236-4244. doi:10.4161/cc.9.20.13636

Sinigaglia, C., Busengdal, H., Leclère, L., Technau, U., Rentzsch, F., 2013. The Bilaterian Head Patterning Gene six3/6 Controls Aboral Domain Development in a Cnidarian. PLoS Biol. 11, e1001488. doi:10.1371/journal.pbio.1001488

Smoyer, C.J., Jaspersen, S.L., 2014. Breaking down the wall: the nuclear envelope during mitosis. Curr. Opin. Cell Biol. 26, 1-9. doi:10.1016/j.ceb.2013.08.002

Snodgrass, R.E., 1960. Facts and Theories on the Insect Head.

Sokoloff, A., 1974. The biology of Tribolium, with special emphasis on genetic aspects.

Stainier, D.Y.R., Kontarakis, Z., Rossi, A., 2015. Making Sense of Anti-Sense Data. Dev. Cell 32, 78. doi:10.1016/j.devcel.2014.12.012

St Johnston, D., 2002. THE ART AND DESIGN OF GENETIC SCREENS: DROSOPHILA MELANOGASTER. Nat. Rev. Genet. 3, 176-188. doi:10.1038/nrg751

Stauber, M., Jackle, H., Schmidt-Ott, U., 1999. The anterior determinant bicoid of Drosophila is a derived Hox class 3 gene. Proc. Natl. Acad. Sci. 96, 3786-3789. doi:10.1073/pnas.96.7.3786

Sternberg, N., Hamilton, D., 1981. Bacteriophage P1 site-specific recombination. J. Mol. Biol. 150, 467-486. doi:10.1016/0022-2836(81)90375-2 
Strobl, F., Schmitz, A., Stelzer, E.H.K., 2015. Live imaging of Tribolium castaneum embryonic development using light-sheet-based fluorescence microscopy. Nat. Protoc. 10, 14861507. doi:10.1038/nprot.2015.093

Strobl, F., Stelzer, E.H.K., 2014. Non-invasive long-term fluorescence live imaging of Tribolium castaneum embryos. Development 141, 2361-2361. doi:10.1242/dev.112706

Tamura, K., Peterson, D., Peterson, N., Stecher, G., Nei, M., Kumar, S., 2011. MEGA5: Molecular Evolutionary Genetics Analysis Using Maximum Likelihood, Evolutionary Distance, and Maximum Parsimony Methods. Mol. Biol. Evol. 28, 2731-2739. doi:10.1093/molbev/msr121

Tautz, D., Friedrich, M., Schröder, R., 1994. Insect embryogenesis - what is ancestral and what is derived? Development 1994, 193-199.

Tomoyasu, Y., Denell, R.E., 2004. Larval RNAi in Tribolium (Coleoptera) for analyzing adult development. Dev. Genes Evol. 214, 575-578. doi:10.1007/s00427-004-0434-0

Tomoyasu, Y., Miller, S.C., Tomita, S., Schoppmeier, M., Grossmann, D., Bucher, G., 2008. Exploring systemic RNA interference in insects: a genome-wide survey for RNAi genes in Tribolium. Genome Biol. 9, R10. doi:10.1186/gb-2008-9-1-r10

Tosches, M.A., Arendt, D., 2013. The bilaterian forebrain: an evolutionary chimaera. Curr. Opin. Neurobiol. 23, 1080-1089. doi:10.1016/j.conb.2013.09.005

Trauner, J., Schinko, J., Lorenzen, M.D., Shippy, T.D., Wimmer, E.A., Beeman, R.W., Klingler, M., Bucher, G., Brown, S.J., 2009. Large-scale insertional mutagenesis of a coleopteran stored grain pest, the red flour beetle Tribolium castaneum, identifies embryonic lethal mutations and enhancer traps. BMC Biol. 7, 73. doi:10.1186/1741-7007-7-73

Tremmel, M., Muller, C., 2013. Insect personality depends on environmental conditions. Behav. Ecol. 24, 386-392. doi:10.1093/beheco/ars175

Tsien, R.Y., 1998. THE GREEN FLUORESCENT PROTEIN. Annu. Rev. Biochem. 67, 509-544. doi:10.1146/annurev.biochem.67.1.509

Tu, Q., Brown, C.T., Davidson, E.H., Oliveri, P., 2006. Sea urchin Forkhead gene family: Phylogeny and embryonic expression. Dev. Biol. 300, 49-62. doi:10.1016/j.ydbio.2006.09.031

Wada, S., 1965. Analyse der Kopf-Hals-Region von Tachycines (Saltatoria) in morphogenetische Einheiten. II. Mitteilung: Experimentell-teratologische Befunde am Kopfskelett mit Berücksichtigung des zentralen Nervensystems. Zool Jb Anat 83, 235-326.

Wagner, G.P., 2007. The developmental genetics of homology. Nat. Rev. Genet. 8, 473-479. doi:10.1038/nrg2099

Walldorf, U., Gehring, W.J., 1992. Empty spiracles, a gap gene containing a homeobox involved in Drosophila head development. EMBO J. 11, 2247-2259.

Weatherbee, S.D., Halder, G., Kim, J., Hudson, A., Carroll, S., 1998. Ultrabithorax regulates genes at several levels of the wing-patterning hierarchy to shape the development of the Drosophila haltere. Genes Dev. 12, 1474-1482. doi:10.1101/gad.12.10.1474

Wei, Z., Yaguchi, J., Yaguchi, S., Angerer, R.C., Angerer, L.M., 2009. The sea urchin animal pole domain is a Six3-dependent neurogenic patterning center. Development 136, 15831583. doi:10.1242/dev.037002

Weigel, D., Jäckle, H., 1990. The fork head domain: a novel DNA binding motif of eukaryotic transcription factors? Cell 63, 455-456.

Weigel, D., Jürgens, G., Küttner, F., Seifert, E., Jäckle, H., 1989. The homeotic gene fork head encodes a nuclear protein and is expressed in the terminal regions of the Drosophila embryo. Cell 57, 645-658. doi:10.1016/0092-8674(89)90133-5

Wheeler, S.R., 2003. The expression and function of the achaete-scute genes in Tribolium castaneum reveals conservation and variation in neural pattern formation and cell fate specification. Development 130, 4373-4381. doi:10.1242/dev.00646 
Wimmer, E.A., Cohen, S.M., Jäckle, H., Desplan, C., 1997. buttonhead does not contribute to a combinatorial code proposed for Drosophila head development. Dev. Camb. Engl. 124, 1509-1517.

Wimmer, E.A., Jäckle, H., Pfeifle, C., Cohen, S.M., 1993. A Drosophila homologue of human Sp1 is a head-specific segmentation gene. Nature 366, 690-694. doi:10.1038/366690a0

Wohlfrom, H., Schinko, J.B., Klingler, M., Bucher, G., 2006. Maintenance of segment and appendage primordia by the Tribolium gene knödel. Mech. Dev. 123, 430-439. doi:10.1016/j.mod.2006.04.003

Yaguchi, J., Angerer, L.M., Inaba, K., Yaguchi, S., 2012. Zinc finger homeobox is required for the differentiation of serotonergic neurons in the sea urchin embryo. Dev. Biol. 363, 74-83. doi:10.1016/j.ydbio.2011.12.024

Yaguchi, S., Yaguchi, J., Angerer, R.C., Angerer, L.M., 2008. A Wnt-FoxQ2-Nodal Pathway Links Primary and Secondary Axis Specification in Sea Urchin Embryos. Dev. Cell 14, 97-107. doi:10.1016/j.devcel.2007.10.012

Yaguchi, S., Yaguchi, J., Wei, Z., Shiba, K., Angerer, L.M., Inaba, K., 2010. ankAT-1 is a novel gene mediating the apical tuft formation in the sea urchin embryo. Dev. Biol. 348, 67-75. doi:10.1016/j.ydbio.2010.09.011

Yaklichkin, S., Vekker, A., Stayrook, S., Lewis, M., Kessler, D.S., 2007. Prevalence of the EH1 Groucho interaction motif in the metazoan Fox family of transcriptional regulators. BMC Genomics 8, 201. doi:10.1186/1471-2164-8-201

Yolov, A.A., Shabarova, Z.A., 1990. Constructing DNA by polymerase recombination. Nucleic Acids Res. 18, 3983-3986. doi:10.1093/nar/18.13.3983

Yon, J., Fried, M., 1989. Precise gene fusion by PCR. Nucleic Acids Res. 17, 4895-4895. doi:10.1093/nar/17.12.4895

Yu, J.-K., Holland, N.D., Holland, L.Z., 2003. AmphiFoxQ2, a novel winged helix/forkhead gene, exclusively marks the anterior end of the amphioxus embryo. Dev. Genes Evol. 213, 102105. doi:10.1007/s00427-003-0302-3

Yu, J.-K., Mazet, F., Chen, Y.-T., Huang, S.-W., Jung, K.-C., Shimeld, S.M., 2008. The Fox genes of Branchiostoma floridae. Dev. Genes Evol. 218, 629-638. doi:10.1007/s00427-008-0229-9

Yusa, K., Zhou, L., Li, M.A., Bradley, A., Craig, N.L., 2011. A hyperactive piggyBac transposase for mammalian applications. Proc. Natl. Acad. Sci. 108, 1531-1536. doi:10.1073/pnas.1008322108

Zettler, L.J., 1991. Pesticide Resistance in Tribolium castaneum and T. confusum (Coleoptera: Tenebrionidae) from Flour Mills in the United States. J. Econ. Entomol. 84, 763-767. doi:10.1093/jee/84.3.763

Zhang, N., Xu, F., Guo, X., 2014. Genomic Analysis of the Pacific Oyster (Crassostrea gigas) Reveals Possible Conservation of Vertebrate Sex Determination in a Mollusc. G3amp58 GenesGenomesGenetics 4, 2207-2217. doi:10.1534/g3.114.013904 


\section{7}

\section{Appendix}

\subsection{General abbreviations}

$\begin{array}{ll}\text { 2A } & \text { self cleaving peptide allowing polycistronic expression } \\ \text { AEL } & \text { after egg laying } \\ \text { AMR } & \text { anterior median region } \\ \text { BCIP } & \text { 5-bromo-4-chloro-3'-indolyl phosphate } \\ \text { bhsp68 } & \text { regulatory region of heat shock protein 68 } \\ \text { bp } & \text { base pair } \\ \text { C3PA-GFP } & \text { 'Cycle 3'mutant of photoactivatable GFP } \\ \text { Cas9 } & \text { CRISPR-associated DNA nuclease9 } \\ \text { CFP } & \text { cyan fluorescent protein } \\ \text { Cre } & \text { 'causes recombination' - recombinase } \\ \text { CRISPR } & \text { clustered regularly interspaced short palindromic repeats } \\ \text { CrPVi1A } & \text { Cricket Paralysis virus extracted RNAi suppressor protein } \\ \text { DAPI } & \text { 4',6-diamidino-2-phenylindole (DNA intercalating fluorescent stain) } \\ \text { DcP-1 } & \text { Cleaved Drosophila death-caspase-1 } \\ \text { DIG } & \text { digoxigenin labeled RNA (for ISH) } \\ \text { DISH } & \text { double-ISH } \\ \text { DNA } & \text { deoxyribonucleic acid } \\ \text { dsRNA } & \text { double-stranded RNA } \\ \text { EFA } & \text { Tc-elongation factor1 } \alpha \text { promoter } \\ \text { EGFP } & \text { enhanced GFP } \\ \text { FLUO } & \text { fluorescin labeled RNA (for ISH) } \\ \text { GFP } & \text { green fluorescent protein } \\ \text { H2Av } & \text { histone H2A variant } \\ \text { HS } & \text { heat shock } \\ \text { hsp68 } & \text { heat shock protein68 } \\ \text { ISH } & \text { in situ hybridization } \\ \text { KFP } & \text { kindling fluorescent protein-1 (based on chromotprotein asulCP) } \\ \text { L1 } & \text { first larval instar } \\ \text { loxP } & \text { locus of crossing over (x), P1 } \\ \text { LSFM } & \text { light-sheet-based fluorescence microscopy } \\ \text { LSM } & \text { laser scanning microscope } \\ \text { m } & \text { prefix, means monomeric (in front of fluorescent proteins) } \\ \text { mRNA } & \text { messenger RNA } \\ n & \text { prefix, indicating a tag with a nuclear localization signal } \\ \text { NBT } & \text { nitro-blue tetrazolium } \\ \text { P } & \text { suffix, indicating that 5' regulatory (promoter) region of a gene was used } \\ \text { PA } & \text { prefix, photoactivatable (in front of fluorescent proteins) } \\ & \end{array}$




\begin{tabular}{|c|c|}
\hline$p B$ & piggyBac (transformation vector) \\
\hline pRNAi & parental RNAi \\
\hline$P U b$ & polyubiquitin \\
\hline RFP & red fluorescent protein \\
\hline RNA & ribonucleic acid \\
\hline RNAi & RNA interference \\
\hline ROI & region of interest \\
\hline rps3 & ribosomal protein subunit 3 \\
\hline$S B$ & San Bernadino (Tribolium wt strain) \\
\hline SV40PolyA & stop/poly adenylation-signal (extracted from Herpes simplex virus) \\
\hline TSA & tyramide signal amplification \\
\hline$v^{w}$ & vermillion $^{\text {white }}$ (Tribolium, eye color deficient strain) \\
\hline & wild-type \\
\hline ת & yellow fluorescent protein \\
\hline Tub1 & $\alpha$ Tubulin1 \\
\hline
\end{tabular}

\subsection{Gene abbreviations}

$\begin{array}{ll}\text { arr } & \text { arrow } \\ \text { ase } & \text { asense } \\ \text { cnc } & \text { cap'n'collar } \\ \text { col } & \text { collier } \\ \text { croc } & \text { crocodile } \\ \text { fkh } & \text { forkhead } \\ \text { foxa } & \text { forkhead box a } \\ \text { foxq2 } & \text { forkhead box q2 } \\ \text { lab } & \text { labial } \\ \text { mib1 } & \text { mindbomb 1 } \\ \text { nk2.1 } & \text { nk2 homeobox 1 (thyroid transcription factor1) } \\ \text { rx } & \text { retinal homeobox } \\ \text { scro } & \text { scarecrow } \\ \text { ser } & \text { serrate } \\ \text { six3 } & \text { sine oculis homeobox homolog 3 } \\ \text { six4 } & \text { sine oculis homeobox homolog } 4 \\ \text { Tc } & \text { prefix, if the gene is a Tribolium ortholog } \\ \text { tll } & \text { tailless } \\ \text { wg } & \text { wingless } \\ \text { wnt1 } & \text { int1 (wingless-related1) }\end{array}$

\subsection{Species}

Clytia hemisphaerica

Nematostella vectensis

Lineus ruber

Platynereis dumerilii

Terebratalia transversa

Priapulus caudatus
(Cnidaria, Medusozoa)

(Cnidaria, Anthozoa)

(Protostomia; Nemertea)

(Protostomia; Annelida)

(Protostomia; Brachiopoda)

(Protostomia; Priapulida) 
Caenorhabditis elegans

Strigamia maritima

Parasteatoda tepidariorum

Tribolium castaneum

Drosophila melanogaster

Strongylocentrotus purpuratus

Saccoglossus kowalevskii

Branchiostoma floridae

Danio rerio

Xenopus laevis

Ornithorhynchus anatinus

Mus musculus
(Protostomia; Nematoda)

(Protostomia; Arthropoda, Myriapoda)

(Protostomia; Arthropoda, Arachnida)

(Protostomia; Arthropoda, Insecta, Coleoptera)

(Protostomia; Arthropoda, Insecta, Diptera)

(Deuterostomia; Echinodermata)

(Deuterostomia; Hemichordata)

(Deuterostomia; Chordata, Cephalochordata (Amphioxus))

(Deuterostomia; Chordata, Actinopterygii)

(Deuterostomia; Chordata, Amphibia)

(Deuterostomia; Chordata, Mammalia (Monotremata)

(Deuterostomia; Chordata, Mammalia (Placentalia))

\subsection{Supplementary tables, figures, sequences, and videos}

\section{$\underline{T c-f o x q 2^{\text {pRNAi }} \text { cuticle phenotype }}$}

Table S7.1 $T$ c-foxq $2^{\text {RNAi_a }}$ general cuticle phenotype using $1 \mu \mathrm{g} / \boldsymbol{\mu l}$ dsRNA in SB.

Pt: Wildtype Unspecific defects Strong defects No cuticle Head defects Total $\mathrm{n}$

$\begin{array}{lllllll}\Sigma & 4 & 0 & 18 & 140 & 676 & 838\end{array}$

$\begin{array}{llllll}\% & 0.5 & 0.0 & 2.1 & 16.7 & 80.7\end{array}$

Table S7.2 Tc-foxq2 ${ }^{\text {RNAi_a }}$ head defects using $1 \mu \mathrm{g} / \mu \mathrm{l}$ dsRNA in SB.

Phenotype: Weak Intermediate Strong

$\begin{array}{llll}\Sigma & 47 & 522 & 107\end{array}$

$\begin{array}{llll}\% & 7.0 & 77.2 & 15.8\end{array}$

Table S7.3 Tc-foxq2 ${ }^{\text {RNAi_b }}$ general cuticle phenotype using $1 \mu \mathrm{g} / \mu \mathrm{l}$ dsRNA in SB.

Pt: Wildtype Unspecific defects Strong defects No cuticle Head defects Total $\mathrm{n}$

$\begin{array}{lllllll}\Sigma & 47 & 4 & 23 & 39 & 200 & 313\end{array}$

$\begin{array}{llllll}\% & 15.0 & 1.3 & 7.3 & 12.5 & 63.9\end{array}$ 
Table S7.4 $\boldsymbol{T} c$-foxq $2^{\text {RNAi_b }}$ head defects using $1 \boldsymbol{\mu g} / \boldsymbol{\mu l}$ dsRNA in SB.

$\begin{array}{lccc}\text { Phenotype: } & \text { Weak } & \text { Intermediate } & \text { Strong } \\ \Sigma & 109 & 73 & 18 \\ \% & 54.5 & 36.5 & 9.0\end{array}$

Table S7.5 Tc-foxq $2^{\text {RNAi_b }}$ general cuticle phenotype using $1 \mu \mathrm{g} / \mu \mathrm{l}$ dsRNA in pBa19 x black.

Pt: Wildtype Unspecific defects Strong defects No cuticle Head defects Total $\mathrm{n}$

$\begin{array}{ccccccc}\Sigma & 0 & 4 & 8 & 12 & 772 & 796 \\ \% & 0.0 & 0.5 & 1.0 & 1.5 & 97.0 & \end{array}$

Table S7.6 Tc-foxq $2^{\text {RNAi_b }}$ head defects using $1 \mu \mathrm{g} / \mu \mathrm{l}$ dsRNA in pBa19 $\times$ black.

Phenotype: Weak Intermediate Strong

$\begin{array}{llll}\Sigma & 8 & 652 & 112\end{array}$

$\begin{array}{llll}\% & 1.0 & 84.5 & 14.5\end{array}$

Quantification of cell death rates in wt and $T c$-foxq $2^{\text {pRNAi }}$ embryos

Table S7.7 Number (pre-normalization) of apoptotic cells per untreated SB embryo.

$\begin{array}{lccr}\text { Stage/Region } & \text { Region 1 } & \text { Region2 } & \text { Region3 } \\ \text { Stage1_1 } & 0 & 0 & 0 \\ \text { Stage1_2 } & 0 & 0 & 0 \\ \text { Stage1_3 } & 0 & 0 & 0 \\ \text { Stage2_1 } & 4 & 3 & 0 \\ \text { Stage2_2 } & 0 & 0 & 0 \\ \text { Stage2_3 } & 4 & 0 & \end{array}$




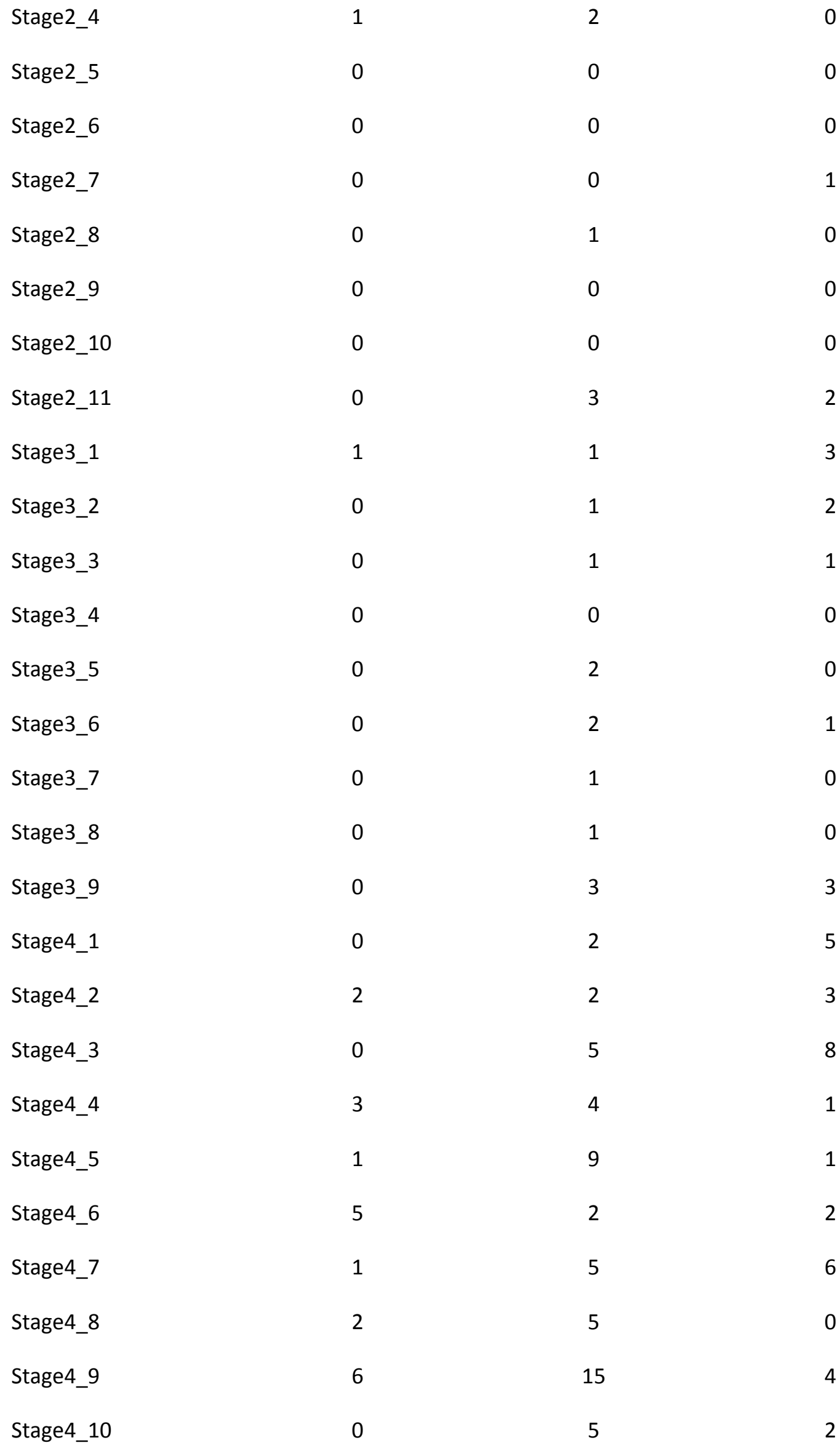




\begin{tabular}{|c|c|c|c|}
\hline Stage4_11 & 5 & 8 & 9 \\
\hline Stage4_12 & 0 & 4 & 1 \\
\hline Stage4_13 & 0 & 1 & 5 \\
\hline Stage4_14 & 4 & 8 & 4 \\
\hline Stage4_15 & 3 & 2 & 0 \\
\hline Stage4_16 & 0 & 8 & 0 \\
\hline Stage4_17 & 1 & 1 & 3 \\
\hline Stage5_1 & 6 & 23 & 22 \\
\hline Stage5_2 & 13 & 7 & 18 \\
\hline Stage5_3 & 14 & 17 & 19 \\
\hline Stage5_4 & 9 & 25 & 17 \\
\hline Stage5_5 & 13 & 29 & 23 \\
\hline Stage5_6 & 10 & 15 & 7 \\
\hline Stage5_7 & 17 & 18 & 21 \\
\hline Stage5_8 & 16 & 15 & 12 \\
\hline Stage5_9 & 23 & 22 & 12 \\
\hline Stage5_10 & 12 & 18 & 16 \\
\hline Stage5_11 & 11 & 14 & 15 \\
\hline Stage5_12 & 1 & 1 & 2 \\
\hline
\end{tabular}

Table S7.8 Number (pre-normalization) of apoptotic cells per Tc-foxq2 ${ }^{\text {pRNAi }}$ embryo.

$\begin{array}{lccr}\text { Stage/Region } & \text { Region 1 } & \text { Region2 } & \text { Region3 } \\ \text { Stage1_1 } & 0 & 0 & 1 \\ \text { Stage1_2 } & 16 & 21 & 36 \\ \text { Stage1_3 } & 0 & 0 & 0 \\ \text { Stage1_4 } & 0 & 0 & 0 \\ \text { Stage1_5 } & 0 & 0 & 0\end{array}$




\begin{tabular}{|c|c|c|c|}
\hline Stage1_6 & 0 & 0 & 0 \\
\hline Stage1_7 & 0 & 0 & 0 \\
\hline Stage2_1 & 0 & 4 & 0 \\
\hline Stage2_2 & 6 & 4 & 13 \\
\hline Stage2_3 & 5 & 3 & 6 \\
\hline Stage2_4 & 0 & 0 & 1 \\
\hline Stage2_5 & 1 & 1 & 1 \\
\hline Stage2_6 & 1 & 8 & 2 \\
\hline Stage2_7 & 0 & 1 & 0 \\
\hline Stage2_8 & 1 & 0 & 0 \\
\hline Stage2_9 & 0 & 1 & 1 \\
\hline Stage2_10 & 4 & 11 & 9 \\
\hline Stage2_11 & 0 & 3 & 1 \\
\hline Stage2_12 & 0 & 0 & 1 \\
\hline Stage3_1 & 1 & 0 & 1 \\
\hline Stage3_2 & 4 & 0 & 4 \\
\hline Stage3_3 & 2 & 8 & 25 \\
\hline Stage3_4 & 0 & 6 & 12 \\
\hline Stage3_5 & 3 & 4 & 3 \\
\hline Stage3_6 & 1 & 2 & 10 \\
\hline Stage3_7 & 0 & 5 & 6 \\
\hline Stage3_8 & 5 & 3 & 1 \\
\hline Stage3_9 & 8 & 10 & 11 \\
\hline Stage3_10 & 9 & 14 & 8 \\
\hline Stage3_11 & 0 & 1 & 5 \\
\hline Stage3_12 & 2 & 0 & 0 \\
\hline Stage3_13 & 25 & 11 & 39 \\
\hline
\end{tabular}




\begin{tabular}{|c|c|c|c|}
\hline Stage3_14 & 0 & 0 & 15 \\
\hline Stage3_15 & 2 & 2 & 7 \\
\hline Stage3_16 & 0 & 0 & 4 \\
\hline Stage3_17 & 0 & 0 & 12 \\
\hline Stage3_18 & 3 & 2 & 4 \\
\hline Stage3_19 & 8 & 4 & 5 \\
\hline Stage4_1 & 17 & 16 & 4 \\
\hline Stage4_2 & 7 & 18 & 15 \\
\hline Stage4_3 & 23 & 17 & 9 \\
\hline Stage4_4 & 7 & 4 & 3 \\
\hline Stage4_5 & 23 & 8 & 12 \\
\hline Stage4_6 & 25 & 24 & 16 \\
\hline Stage4_7 & 13 & 6 & 5 \\
\hline Stage4_8 & 6 & 34 & 14 \\
\hline Stage4_9 & 11 & 23 & 10 \\
\hline Stage4_10 & 19 & 16 & 8 \\
\hline Stage4_11 & 38 & 18 & 10 \\
\hline Stage4_12 & 18 & 14 & 7 \\
\hline Stage4_13 & 8 & 10 & 10 \\
\hline Stage4_14 & 22 & 25 & 13 \\
\hline Stage4_15 & 8 & 10 & 3 \\
\hline Stage5_1 & 27 & 89 & 40 \\
\hline Stage5_2 & 14 & 36 & 14 \\
\hline Stage5_3 & 10 & 18 & 5 \\
\hline Stage5_4 & 14 & 24 & 13 \\
\hline Stage5_5 & 19 & 57 & 46 \\
\hline Stage5_6 & 23 & 38 & 4 \\
\hline
\end{tabular}


N $\stackrel{m}{m} \vec{m}$

กั ช

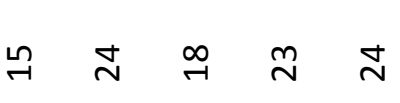

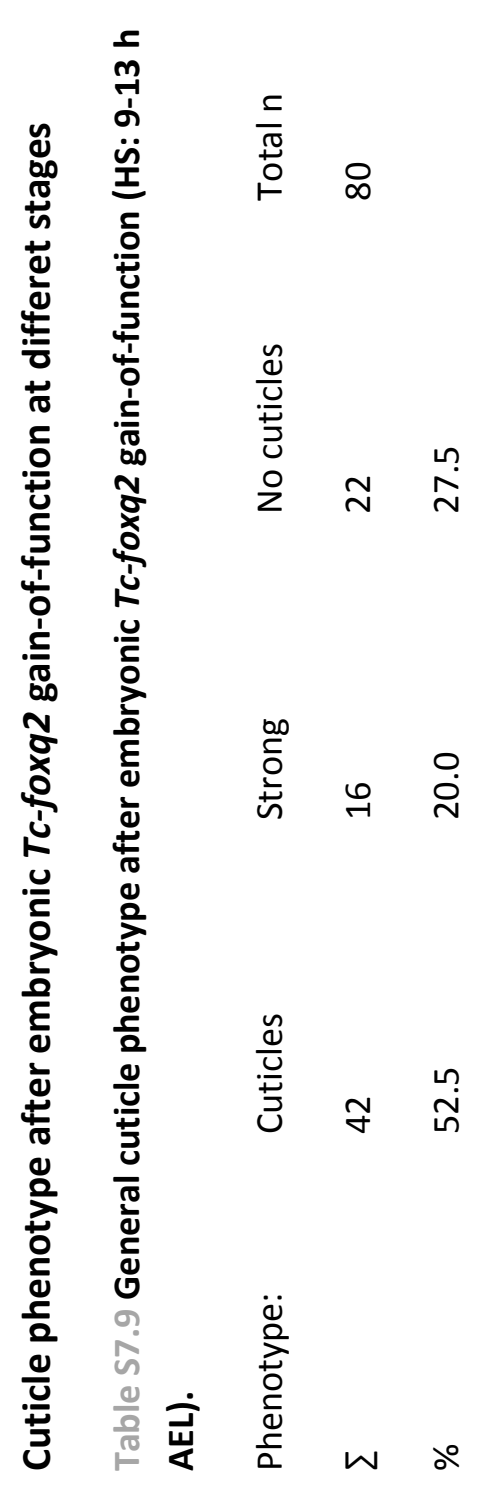

\begin{tabular}{|c|c|c|c|c|}
\hline Pygo & 14.3 & 27.4 & 0.0 & 0.0 \\
\hline Uro & 7.1 & 20.2 & 0.0 & 0.0 \\
\hline AS1-8 & 19.0 & 35.7 & 9.5 & 0.0 \\
\hline LP1-3 & 3.6 & 64.3 & 14.3 & 0.0 \\
\hline TS1-3 & 4.8 & 59.5 & 0.0 & 0.0 \\
\hline Lb & 9.5 & 76.2 & 0.0 & 0.0 \\
\hline Mx & 10.7 & 64.3 & 0.0 & 0.0 \\
\hline Md & 10.7 & 63.1 & 0.0 & 0.0 \\
\hline AtF & 2.4 & 13.1 & 0.0 & 1.2 \\
\hline At & 0.0 & 3.6 & 0.0 & 0.0 \\
\hline br & 0.0 & 3.6 & 0.0 & 0.0 \\
\hline brB & 0.0 & 8.3 & 0.0 & 0.0 \\
\hline pMES & 0.0 & 14.3 & 0.0 & 0.0 \\
\hline mMES & 0.0 & 13.1 & 0.0 & 0.0 \\
\hline aMES & 0.0 & 13.1 & 0.0 & 0.0 \\
\hline pGTS & 0.0 & 4.8 & 0.0 & 0.0 \\
\hline dGTS & 0.0 & 3.6 & 0.0 & 4.8 \\
\hline aGTS & 0.0 & 3.6 & 0.0 & 1.2 \\
\hline ABB & 0.0 & 32.1 & 0.0 & 0.0 \\
\hline pVTS & 0.0 & 14.3 & 0.0 & 3.6 \\
\hline mVTS & 0.0 & 9.5 & 0.0 & 0.0 \\
\hline avTs & 0.0 & 11.9 & 0.0 & 0.0 \\
\hline IrS & 0.0 & 6.0 & 0.0 & 0.0 \\
\hline cls & 0.0 & 11.9 & 0.0 & 0.0 \\
\hline $\mathbf{L r}$ & 11.9 & 3.6 & 0.0 & 0.0 \\
\hline$\% /$ Structure & Deformed & Absent & Fused & Duplicated \\
\hline
\end{tabular}




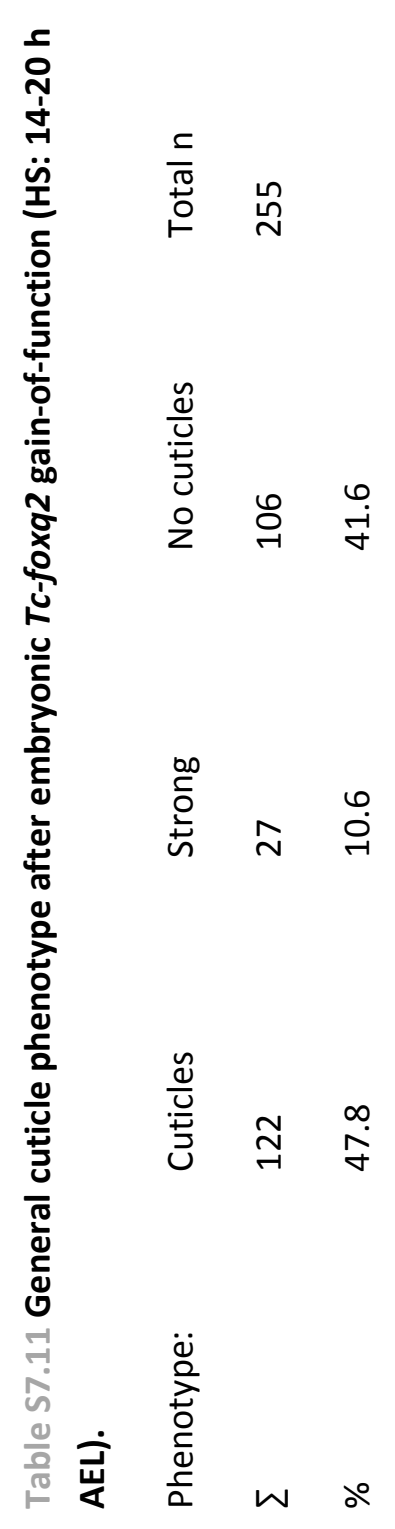

\begin{tabular}{|c|c|c|c|c|}
\hline Pygo & 1.6 & 93.4 & 0.0 & 0.0 \\
\hline Uro & 5.7 & 79.9 & 1.6 & 0.0 \\
\hline AS1-8 & 0.8 & 27.9 & 67.2 & 0.0 \\
\hline LP1-3 & 4.9 & 11.1 & 49.6 & 0.0 \\
\hline TS1-3 & 0.4 & 8.2 & 0.8 & 0.0 \\
\hline Lb & 0.8 & 0.8 & 0.0 & 0.0 \\
\hline Mx & 0.8 & 0.0 & 0.0 & 0.0 \\
\hline Md & 0.0 & 0.8 & 0.0 & 0.0 \\
\hline AtF & 16.0 & 43.4 & 0.0 & 0.4 \\
\hline At & 1.2 & 0.8 & 0.0 & 0.0 \\
\hline br & 0.0 & 1.2 & 0.0 & 0.0 \\
\hline brB & 0.0 & 3.3 & 0.0 & 0.0 \\
\hline pMES & 0.0 & 1.6 & 0.0 & 0.0 \\
\hline mMES & 0.0 & 2.5 & 0.0 & 0.0 \\
\hline aMES & 0.0 & 1.6 & 0.0 & 0.8 \\
\hline pGTS & 0.0 & 0.4 & 0.0 & 0.4 \\
\hline dGTS & 0.0 & 1.2 & 0.0 & 3.7 \\
\hline aGTS & 0.0 & 1.6 & 0.0 & 1.2 \\
\hline ABB & 0.0 & 21.7 & 0.0 & 0.8 \\
\hline pVTS & 0.0 & 0.8 & 0.0 & 7.8 \\
\hline mVTs & 0.0 & 49.2 & 0.0 & 0.8 \\
\hline aVTS & 0.0 & 8.2 & 0.0 & 0.0 \\
\hline IrS & 0.0 & 0.0 & 0.0 & 0.4 \\
\hline cls & 0.0 & 0.0 & 0.0 & 0.0 \\
\hline $\mathbf{L r}$ & 0.0 & 0.0 & 0.0 & 0.0 \\
\hline$\% /$ Structure & Deformed & Absent & Fused & Duplicated \\
\hline
\end{tabular}

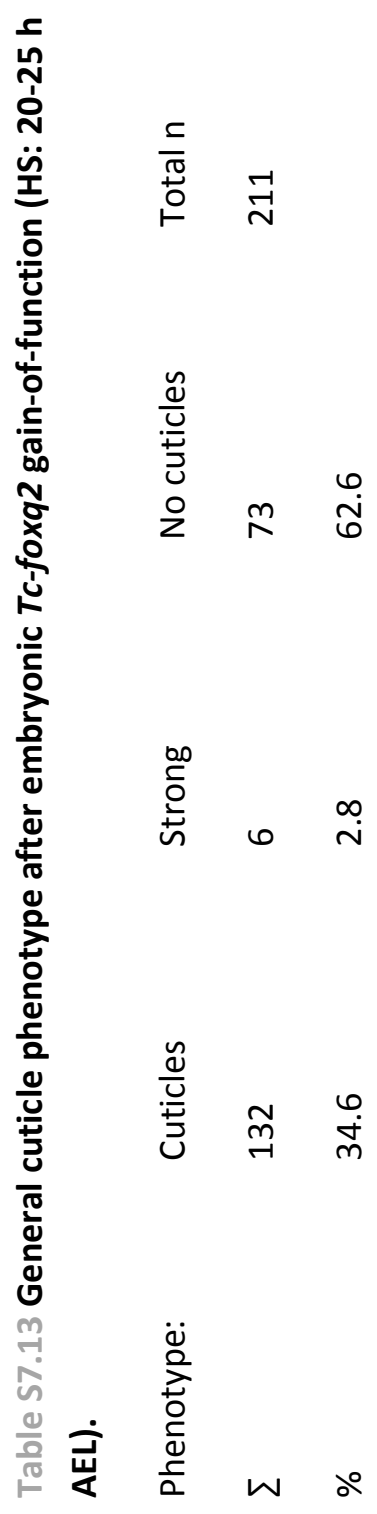




\begin{tabular}{|c|c|c|c|c|}
\hline Pygo & 9.8 & 81.4 & 0.0 & 0.8 \\
\hline Uro & 13.6 & 55.3 & 0.0 & 9.1 \\
\hline AS1-8 & 4.5 & 5.3 & 79.9 & 0.0 \\
\hline LP1-3 & 1.9 & 0.8 & 42.0 & 0.0 \\
\hline TS1-3 & 0.0 & 0.0 & 0.0 & 0.0 \\
\hline Lb & 4.5 & 0.8 & 0.0 & 0.0 \\
\hline $\mathbf{M x}$ & 4.9 & 0.0 & 0.0 & 0.0 \\
\hline Md & 2.7 & 0.4 & 0.0 & 0.0 \\
\hline AtF & 2.7 & 33.3 & 0.0 & 0.0 \\
\hline At & 0.8 & 0.0 & 0.0 & 0.0 \\
\hline br & 0.0 & 0.0 & 0.0 & 0.0 \\
\hline brB & 0.0 & 2.3 & 0.0 & 0.0 \\
\hline pMES & 0.0 & 3.0 & 0.0 & 0.0 \\
\hline mMES & 0.0 & 2.7 & 0.0 & 0.0 \\
\hline aMES & 0.0 & 1.9 & 0.0 & 0.8 \\
\hline pGTS & 0.0 & 0.8 & 0.0 & 0.0 \\
\hline dGTS & 0.0 & 1.1 & 0.0 & 1.5 \\
\hline aGTS & 0.0 & 1.5 & 0.0 & 0.8 \\
\hline ABB & 0.0 & 26.1 & 0.0 & 0.0 \\
\hline pVTS & 0.0 & 0.0 & 0.0 & 5.3 \\
\hline mVTS & 0.0 & 38.3 & 0.0 & 0.4 \\
\hline aVTS & 0.4 & 6.8 & 0.0 & 0.4 \\
\hline IrS & 0.0 & 1.1 & 0.0 & 0.4 \\
\hline clS & 0.0 & 1.1 & 0.0 & 1.1 \\
\hline Lr & 3.8 & 0.0 & 0.0 & 0.0 \\
\hline$\% /$ Structure & Deformed & Absent & Fused & Duplicated \\
\hline
\end{tabular}




\section{Vector maps and sequences}

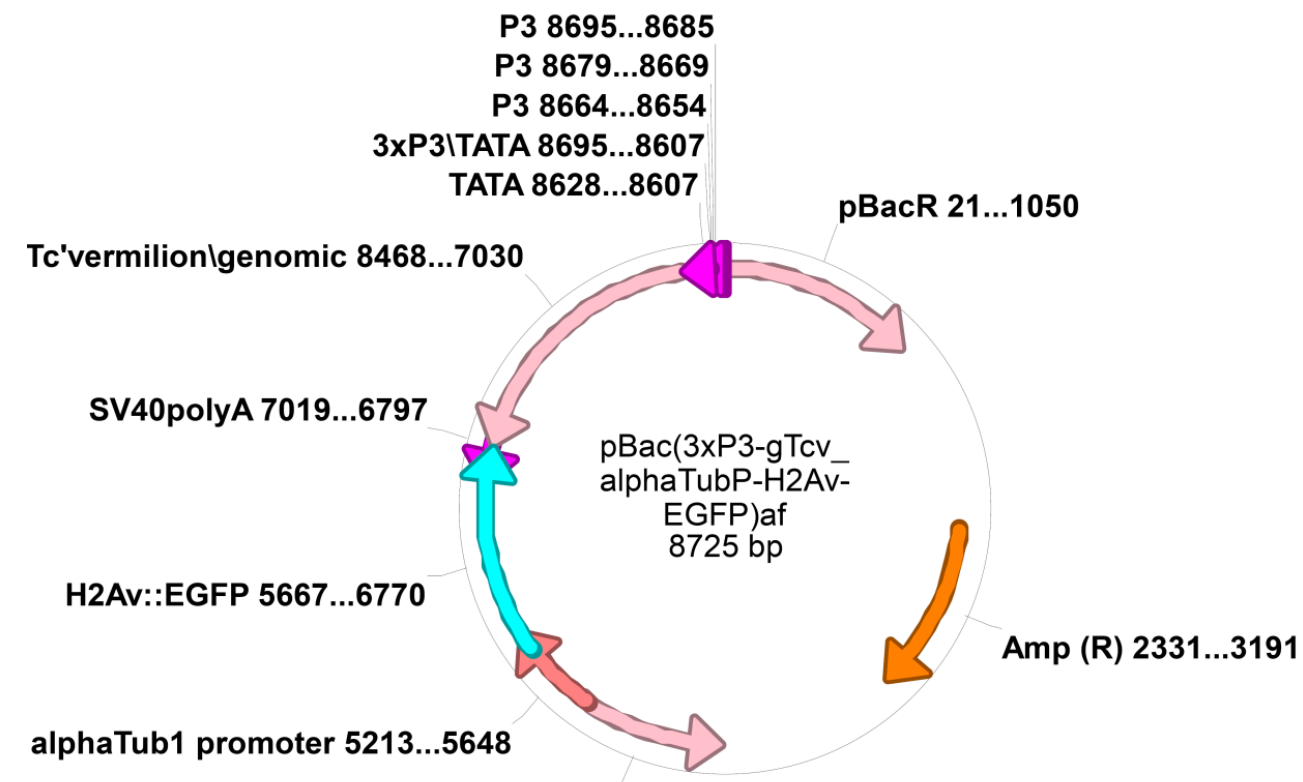

pBacL 5206...4489

Figure S7.15 pB[3xP3-gTc'v;Tc' $\boldsymbol{\alpha T u b 1 P - T c ^ { \prime } H 2 A v : : E G F P ] . ~}$

Sequence S7.16 pB[3xP3-gTc'v;Tc' $\alpha$ Tub1P- Tc'H2Av::EGFP], GenBank formatted.

LOCUS pBac_3xP3_gTcv_alp 8725 bp ds-DNA circular 05-MAY-2016

SOURCE

ORGANISM

COMMENT

COMMENT ApEinfo:methylated:1

FEATURES Location/Qualifiers

promoter complement(8607..8695)

/vntifkey="29"

/label=3×P3\TATA

/ApEinfo_fwdcolor=\#ffffff

/ApEinfo_revcolor=SystemWindow

misc_feature complement(6797..7019)

/vntifkey="21"

/label=SV40polyA

/ApEinfo_fwdcolor=\#ff8080

/ApEinfo_revcolor=\#ffOOff

misc structure 21..1050

/vntifkey="88"

/label=pBacR

/ApEinfo_fwdcolor=pink

/ApEinfo_revcolor=pink

misc_structure complement(4489..5206)

/vntifkey="88"

/label=pBacL

/ApEinfo_fwdcolor=pink

/ApEinfo_revcolor=pink

misc_signal complement(7030..8468)

/vntifkey="87"

/label=Tc'vermilion\genomic

/ApEinfo_fwdcolor=pink

/ApEinfo_revcolor=pink

misc_feature complement(8685..8695)

/vntifkey="21" 


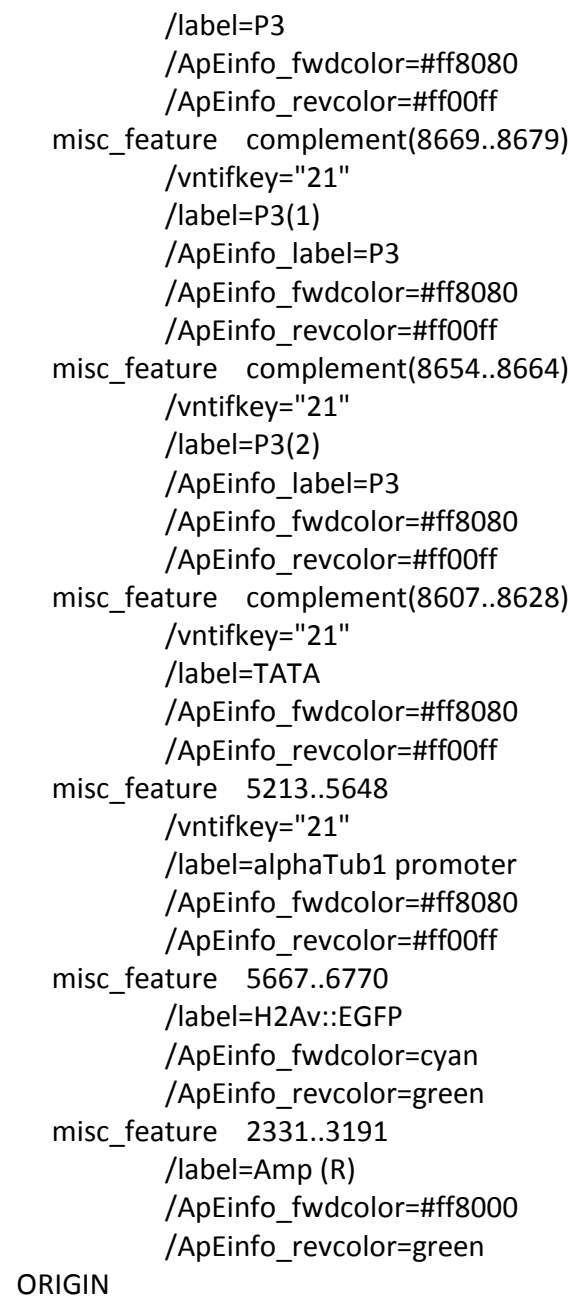

1 cattgtggga accgtgcgat caaacaaacg cgagataccg gaagtactga aaaacagtcg 61 ctccaggcca gtgggaacat cgatgttttg ttttgacgga ccccttactc tcgtctcata 121 taaaccgaag ccagctaaga tggtatactt attatcatct tgtgatgagg atgcttctat 181 caacgaaagt accggtaaac cgcaaatggt tatgtattat aatcaaacta aaggcggagt 241 ggacacgcta gaccaaatgt gttctgtgat gacctgcagt aggaagacga ataggtggcc 301 tatggcatta ttgtacggaa tgataaacat tgcctgcata aattctttta ttatatacag 361 ccataatgtc agtagcaagg gagaaaaggt tcaaagtcgc aaaaaattta tgagaaacct 421 ttacatgagc ctgacgtcat cgtttatgcg taagcgttta gaagctccta ctttgaagag 481 atatttgcgc gataatatct ctaatatttt gccaaatgaa gtgcctggta catcagatga 541 cagtactgaa gagccagtaa tgaaaaaacg tacttactgt acttactgcc cctctaaaat 601 aaggcgaaag gcaaatgcat cgtgcaaaaa atgcaaaaaa gttatttgtc gagagcataa 661 tattgatatg tgccaaagtt gtttctgact gactaataag tataatttgt ttctattatg 721 tataagttaa gctaattact tattttataa tacaacatga ctgtttttaa agtacaaaat 781 aagtttattt ttgtaaaaga gagaatgttt aaaagttttg ttactttata gaagaaattt 841 tgagtttttg ttttttttta ataaataaat aaacataaat aaattgtttg ttgaatttat 901 tattagtatg taagtgtaaa tataataaaa cttaatatct attcaaatta ataaataaac 961 ctcgatatac agaccgataa aacacatgcg tcaattttac gcatgattat ctttaacgta 1021 cgtcacaata tgattatctt tctagggtta aataatagtt tctaattttt ttattattca 1081 gcctgctgtc gtgaataccg tatatctcaa cgctgtctgt gagattgtcg tattctagcc $1141 \mathrm{tttttagttt}$ ttcgctcatc gacttgatat tgtccgacac attttcgtcg atttgcgttt 1201 tgatcaaaga cttgagcaga gacacgttaa tcaactgttc aaattgatcc atattaacga 1261 tatcaacccg atgcgtatat ggtgcgtaaa atatattttt taaccctctt atactttgca 1321 ctctgcgtta atacgcgttc gtgtacagac gtaatcatgt tttctttttt ggataaaact 1381 cctactgagt ttgacctcat attagaccct cacaagttgc aaaacgtggc attttttacc 1441 aatgaagaat ttaaagttat tttaaaaaat ttcatcacag atttaaagaa gaaccaaaaa 1501 ttaaattatt taatcgacca gttaatcaac gtgtacacag agcgcaaaaa acacgcagcc 1561 cgacgtgttg gctaaaatta ttaaatcaac ttgtgttata gtcacgattt gccgtccaac 1621 gtgttcctca aaaagttgaa gaccaacaag tttacggaca ctagttaatt atttgatttt 1681 gccccacttc attttgtggg atcacaattt tgttatattt taaacaaagc ttggcactgg 
1741 ccgtcgtttt acaacgtcgt gactgggaaa accctggcgt tacccaactt aatcgccttg 1801 cagcacatcc ccctttcgcc agctggcgta atagcgaaga ggcccgcacc gatcgccctt 1861 cccaacagtt gcgcagcctg aatggcgaat ggcgcctgat gcggtatttt ctccttacgc 1921 atctgtgcgg tatttcacac cgcatatggt gcactctcag tacaatctgc tctgatgccg 1981 catagttaag ccagccccga cacccgccaa cacccgctga cgcgccctga cgggcttgtc 2041 tgctcccggc atccgcttac agacaagctg tgaccgtctc cgggagctgc atgtgtcaga 2101 ggttttcacc gtcatcaccg aaacgcgcga gacgaaaggg cctcgtgata cgcctatttt 2161 tataggttaa tgtcatgata ataatggttt cttagacgtc aggtggcact tttcggggaa 2221 atgtgcgcgg aacccctatt tgtttatttt tctaaataca ttcaaatatg tatccgctca 2281 tgagacaata accctgataa atgcttcaat aatattgaaa aaggaagagt atgagtattc 2341 aacatttccg tgtcgccctt attccctttt ttgcggcatt ttgccttcct gtttttgctc 2401 acccagaaac gctggtgaaa gtaaaagatg ctgaagatca gttgggtgca cgagtgggtt 2461 acatcgaact ggatctcaac agcggtaaga tccttgagag ttttcgcccc gaagaacgtt 2521 ttccaatgat gagcactttt aaagttctgc tatgtggcgc ggtattatcc cgtattgacg 2581 ccgggcaaga gcaactcggt cgccgcatac actattctca gaatgacttg gttgagtact 2641 caccagtcac agaaaagcat cttacggatg gcatgacagt aagagaatta tgcagtgctg 2701 ccataaccat gagtgataac actgcggcca acttacttct gacaacgatc ggaggaccga 2761 aggagctaac cgcttttttg cacaacatgg gggatcatgt aactcgcctt gatcgttggg 2821 aaccggagct gaatgaagcc ataccaaacg acgagcgtga caccacgatg cctgtagcaa 2881 tggcaacaac gttgcgcaaa ctattaactg gcgaactact tactctagct tcccggcaac 2941 aattaataga ctggatggag gcggataaag ttgcaggacc acttctgcgc tcggcccttc 3001 cggctggctg gtttattgct gataaatctg gagccggtga gcgtgggtct cgcggtatca 3061 ttgcagcact ggggccagat ggtaagccct cccgtatcgt agttatctac acgacgggga 3121 gtcaggcaac tatggatgaa cgaaatagac agatcgctga gataggtgcc tcactgatta 3181 agcattggta actgtcagac caagtttact catatatact ttagattgat ttaaaacttc 3241 atttttaatt taaaaggatc taggtgaaga tcctttttga taatctcatg accaaaatcc 3301 cttaacgtga gttttcgttc cactgagcgt cagaccccgt agaaaagatc aaaggatctt 3361 cttgagatcc tttttttctg cgcgtaatct gctgcttgca aacaaaaaaa ccaccgctac 3421 cagcggtggt ttgtttgccg gatcaagagc taccaactct ttttccgaag gtaactggct 3481 tcagcagagc gcagatacca aatactgtcc ttctagtgta gccgtagtta ggccaccact 3541 tcaagaactc tgtagcaccg cctacatacc tcgctctgct aatcctgtta ccagtggctg 3601 ctgccagtgg cgataagtcg tgtcttaccg ggttggactc aagacgatag ttaccggata 3661 aggcgcagcg gtcgggctga acggggggtt cgtgcacaca gcccagcttg gagcgaacga 3721 cctacaccga actgagatac ctacagcgtg agcattgaga aagcgccacg cttcccgaag 3781 ggagaaaggc ggacaggtat ccggtaagcg gcagggtcgg aacaggagag cgcacgaggg 3841 agcttccagg gggaaacgcc tggtatcttt atagtcctgt cgggtttcgc cacctctgac 3901 ttgagcgtcg atttttgtga tgctcgtcag gggggcggag cctatggaaa aacgccagca 3961 acgcggcctt tttacggttc ctggcctttt gctggccttt tgctcacatg ttctttcctg 4021 cgttatcccc tgattctgtg gataaccgta ttaccgcctt tgagtgagct gataccgctc 4081 gccgcagccg aacgaccgag cgcagcgagt cagtgagcga ggaagcggaa gagcgcccaa 4141 tacgcaaacc gcctctcccc gcgcgttggc cgattcatta atgcagctgg cacgacaggt 4201 ttcccgactg gaaagcgggc agtgagcgca acgcaattaa tgtgagttag ctcactcatt 4261 aggcacccca ggctttacac tttatgcttc cggctcgtat gttgtgtgga attgtgagcg 4321 gataacaatt tcacacagga aacagctatg accatgatta cgaattcgag ctcggtaccc 4381 ggggatcctc tagagtcgac gctcgcgcga cttggtttgc cattctttag cgcgcgtcgc 4441 gtcacacagc ttggccacaa tgtggttttt gtcaaacgaa gattctatga cgtgtttaaa 4501 gtttaggtcg agtaaagcgc aaatcttttt taaccctaga aagatagtct gcgtaaaatt 4561 gacgcatgca ttcttgaaat attgctctct ctttctaaat agcgcgaatc cgtcgctgtg 4621 catttaggac atctcagtcg ccgcttggag ctcccgtgag gcgtgcttgt caatgcggta 4681 agtgtcactg attttgaact ataacgaccg cgtgagtcaa aatgacgcat gattatcttt 4741 tacgtgactt ttaagattta actcatacga taattatatt gttatttcat gttctactta 4801 cgtgataact tattatatat atatttctt gttatagata tcgtgactaa tatataataa 4861 aatgggtagt tctttagacg atgagcatat cctctctgct cttctgcaaa gcgatgacga 4921 gcttgttggt gaggattctg acagtgaaat atcagatcac gtaagtgaag atgacgtcca 4981 gagcgataca gaagaagcgt ttatagatga ggtacatgaa gtgcagccaa cgtcaagcgg 5041 tagtgaaata ttagacgaac aaaatgttat tgaacaacca ggttcttcat tggcttctaa 5101 cagaatcttg accttgccac agaggactat tagaggtaag aataaacatt gttggtcaac 5161 ttcaaagtcc acgaggcgta gccgagtctc tgcactgaac attgtcagat ctcactgcag 5221 tgaacggtta tgatggtgca acgaccggga cctcagctgt ataaaaaatc tgaaaataat 5281 tgataatggt cgaagaccat aaggaaaaat aattcgaaaa ttaaaggcaa agtatgaaaa 5341 tgctggaaat aaatcgggaa acaccgaatt tttggtcaca ggaaccaacg aatttttttt 5401 tgaatattga tttgaaaaga gatcgagaac aaggataaca atcttccgtt agagaccgtt 5461 ggtaccgaca tggcggggag ccaatcagaa gtctggaaat cgggcgttgg ttgctgacgt 
5521 caacaagctt ataaatagca acgacagttg aaaatcgaat caaagtcgtt tggaaaaagc

5581 cagagcttgt atttccgaag cgtactcccg tttttctgct cttttgtggt gtaatttgta

5641 aaactcaagg ccggccCTAA AGAAAAATGG CTGGTGGCAA AGCAGGGAAA GATTCGGGCA

5701 AAGCTAAAGC CAAGGCGGTG TCGCGCTCGG CGCGTGCCGG ACTCCAGTTC CCCGTGGGGA

5761 GGATCCACCG CCACTTGAAG AATCGCACTA CGAGCCATGG ACGCGTGGGG GCCACCGCGG 5821 CTGTGTATTC CGCCGCGATT TTGGAGTATT TGACAGCTGA GGTCCTGGAG TTGGCCGGGA 5881 ACGCCAGTAA GGACTTGAAG GTGAAGAGGA TCACCCCCAG GCACTTGCAG CTGGCCATCA 5941 GAGGGGATGA GGAGCTGGAC AGCCTGATCA AGGCCACTAT CGCCGGCGGA GGCGTCATTC 6001 CACACATCCA CAAGAGTTTG ATCGGGAAAA AGGGCCACTC ACAGCCCGTC ATGGTGAGCA 6061 AGGGCGAGGA GCTGTTCACC GGGGTGGTGC CCATCCTGGT CGAGCTGGAC GGCGACGTAA 6121 ACGGCCACAA GTTCAGCGTG TCCGGCGAGG GCGAGGGCGA TGCCACCTAC GGCAAGCTGA 6181 CCCTGAAGTT CATCTGCACC ACCGGCAAGC TGCCCGTGCC CTGGCCCACC CTCGTGACCA 6241 CCCTGACCTA CGGCGTGCAG TGCTTCAGCC GCTACCCCGA CCACATGAAG CAGCACGACT 6301 TCTTCAAGTC CGCCATGCCC GAAGGCTACG TCCAGGAGCG CACCATCTTC TTCAAGGACG 6361 ACGGCAACTA CAAGACCCGC GCCGAGGTGA AGTTCGAGGG CGACACCCTG GTGAACCGCA 6421 TCGAGCTGAA GGGCATCGAC TTCAAGGAGG ACGGCAACAT CCTGGGGCAC AAGCTGGAGT 6481 ACAACTACAA CAGCCACAAC GTCTATATCA TGGCCGACAA GCAGAAGAAC GGCATCAAGG 6541 TGAACTTCAA GATCCGCCAC AACATCGAGG ACGGCAGCGT GCAGCTCGCC GACCACTACC 6601 AGCAGAACAC CCCCATCGGC GACGGCCCCG TGCTGCTGCC CGACAACCAC TACCTGAGCA 6661 CCCAGTCCGC CCTGAGCAAA GACCCCAACG AGAAGCGCGA TCACATGGTC CTGCTGGAGT 6721 TCGTGACCGC CGCCGGGATC ACTCTCGGCA TGGACGAGCT GTACAAGTAA ggcgcgccgt

6781 acgcgatcgt aagcttgatg agtttggaca aaccacaact agaatgcagt gaaaaaaatg 6841 ctttatttgt gaaatttgtg atgctattgc tttatttgta accattataa gctgcaataa 6901 acaagttaac aacaacaatt gcattcattt tatgtttcag gttcaggggg aagtgtggga 6961 ggttttttaa agcaagtaaa acctctacaa atgtggtatg gctgattatg atctagagtc 7021 gcggccgcct taacgaaata attaattgtt tccattcgac acgatattgg tgctatttgc 7081 cgacccccaa ttgcacaaat gacttctcat agacgtagac aaggggggga tgtaggaccg 7141 tggtatcaaa aacgtcgata aattgaaaag gtccacgaaa actttatacc gatcgctgaa 7201 aaacaaaatc aaacattgca aaaaccgtgt tgaaatcaat ttcaccttaa cgttgacctt 7261 aaatactgat accccgaaga gccccctgtt cctaactgcg aagaaccaat cattctttgc 7321 accatcaaaa catgattatc tgaaattata aaatttaaac aaaaacaatt gacgtacgga 7381 tttactaaca ccgccatttc gtgatcagcg agtctatgtc catcaaaagt gttagaattt 7441 ggtggggctg actgaacctg ggttcgtccc tgtaaaaggt gatcatgata gccccttgca 7501 gggctttgtg tgagaagcgc ctctcgccgc gcgacattag agcctcatgg acctcaactt 7561 taaaaatcga ctcgtaaacc tcgcgtctct tctccaaatc gttcaatttg taacgcttta 7621 aagtctccgc ctcctccttc tccgccagct ctttttgctc ggttaaaagc ttctcgaccg 7681 ctttttggta cttcccccag aaattaaacc cctcgagctc cagccccggc gttcgctcca 7741 gccaccgctg gaccaaatcg gtcaaagacg gctctttctc cgacttggcg atttgctcga 7801 gcgctttttc gtcatttccg aacactttcg agtagttctg gttgtatttg acgcggtttt 7861 cctggcgcac cccgagcttg ttttccagga gcctgaactg caggctctgg aacccggaag 7921 cgggccgcag ataacaccgg aagtccatga aatcgagcgg tgtcatcgtt tccagaatca 7981 tcacttggtc caccaagacc tacaattgcg acagttttat ctcgtgaccg caatttgttg 8041 cgaaattgtg aaccttgagg atcaaaacaa cacgattgag gcgtttgagg atttccaaag 8101 tttgcgactc ttctaaaacg tcgctgaaaa tgttgcggat cgagtccagc tcgtagatta 8161 tttgtttgaa ccataactca tacgctggaa ttacttgtta tagttaatta cggttaagtg 8221 ggttttttac cttgatgggt cacaataaat aaatgctcgt cgtgcacggg ctggttattt 8281 tgctcgctga gtaatctttg ggcttctaaa atcttgtcca acatcaagta ttcaccgtaa 8341 agcatcccac attcttcgct cagttggtcg ccttcttggg cttcacttaa tttacgatta 8401 attgtaaatg gaataattaa attaaatcat acttacgagg gtctcagtgg gcaactcatt 8461 acgaccgcgg taccgtcgac gttctagcgg taccccgatt gtttagcttg ttcagctgcg 8521 cttgtttatt tgcttagctt tcgcttagcg acgtgttcac tttgcttgtt tgaattgaat 8581 tgtcgctccg tagacgaagc gcctctattt atactccggc ggtcgagggt tcgaaatcga 8641 taagcttgga tcctaattga attagctcta attgaattag tctctaattg aattagatcc // 8701 ccgggcgagc tcgaattaat tcaac 
P3 8959...8949

P3 8943...8933

P3 8928...8918

3XP3ITATA $8959 \ldots 8871$

TATA $8892 \ldots 8871$

pBacR 21...1050

Tc'vermilionlgenomic $8732 . . .7294$

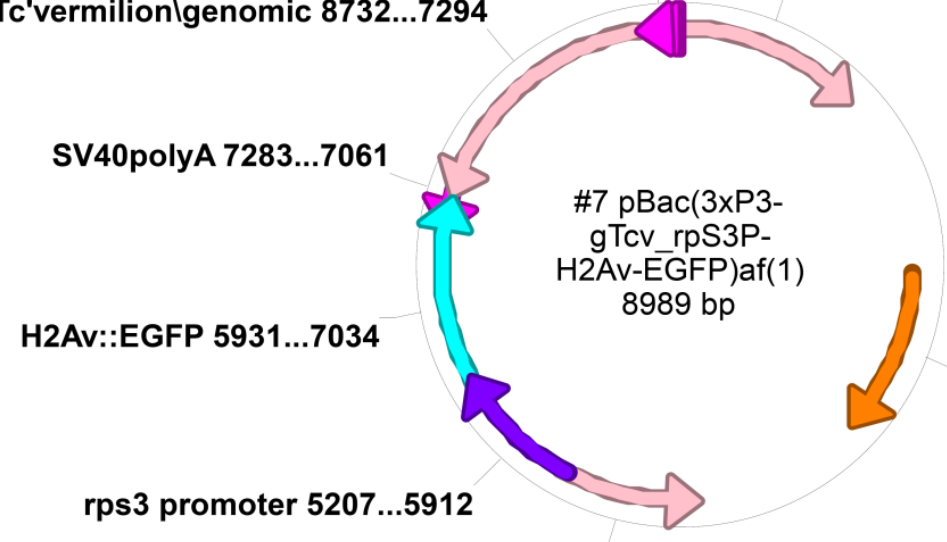

Amp (R) 2331 ...3191

pBacL 5206...4489

Figure S7.17 pB[3xP3-gTc'v;Tc'rpS3P-Tc'H2Av::EGFP].

Sequence S7.18 pB[3xP3-gTc'v;Tc'rpS3P-Tc'H2Av::EGFP], GenBank formatted.

LOCUS _7_pBac_3xP3_gTcv_ 8989 bp ds-DNA circular 05-MAY-2016

SOURCE

ORGANISM

COMMENT

COMMENT ApEinfo:methylated:1

FEATURES Location/Qualifiers

promoter complement(8871..8959)

/vntifkey="29"

/label=3xP3\TATA

/ApEinfo_fwdcolor=\#ffffff

/ApEinfo revcolor=SystemWindow

misc_feature complement(7061..7283)

/vntifkey="21"

/label=SV40polyA

/ApEinfo_fwdcolor=\#ff8080

/ApEinfo_revcolor=\#ffooff

misc structure $21 . .1050$

/vntifkey="88"

/label=pBacR

/ApEinfo fwdcolor=pink

/ApEinfo_revcolor=pink

misc_structure complement(4489..5206)

/vntifkey="88"

/label=pBacL

/ApEinfo_fwdcolor=pink

/ApEinfo_revcolor=pink

misc_signal complement(7294..8732)

/vntifkey="87"

/label=Tc'vermilion\genomic

/ApEinfo_fwdcolor=pink

/ApEinfo_revcolor=pink

misc_feature complement(8949..8959)

/vntifkey="21"

/label=P3 
/ApEinfo_fwdcolor=\#ff8080

/ApEinfo_revcolor=\#ffOOff

misc_feature complement(8933..8943)

/vntifkey="21"

/label=P3(1)

/ApEinfo_label=P3

/ApEinfo_fwdcolor=\#ff8080

/ApEinfo_revcolor=\#ffOOff

misc_feature complement(8918..8928)

/vntifkey="21"

/label=P3(2)

/ApEinfo_label=P3

/ApEinfo_fwdcolor=\#ff8080

/ApEinfo_revcolor=\#ffOOff

misc_feature complement(8871..8892)

/vntifkey="21"

/label=TATA

/ApEinfo_fwdcolor=\#ff8080

/ApEinfo_revcolor=\#ffooff

misc_feature 5931..7034

/label=H2Av::EGFP

/ApEinfo_fwdcolor=cyan

/ApEinfo_revcolor=green

misc_feature 5207..5912

/label=rps3 promoter

/ApEinfo_fwdcolor=\#8000ff

/ApEinfo_revcolor=green

misc_feature 2331..3191

/label=Amp (R)

/ApEinfo_fwdcolor=\#ff8000

/ApEinfo_revcolor=green

ORIGIN

1 cattgtggga accgtgcgat caaacaaacg cgagataccg gaagtactga aaaacagtcg 61 ctccaggcca gtgggaacat cgatgttttg ttttgacgga ccccttactc tcgtctcata 121 taaaccgaag ccagctaaga tggtatactt attatcatct tgtgatgagg atgcttctat 181 caacgaaagt accggtaaac cgcaaatggt tatgtattat aatcaaacta aaggcggagt 241 ggacacgcta gaccaaatgt gttctgtgat gacctgcagt aggaagacga ataggtggcc 301 tatggcatta ttgtacggaa tgataaacat tgcctgcata aattcttta ttatatacag 361 ccataatgtc agtagcaagg gagaaaaggt tcaaagtcgc aaaaaattta tgagaaacct 421 ttacatgagc ctgacgtcat cgtttatgcg taagcgttta gaagctccta ctttgaagag 481 atatttgcgc gataatatct ctaatatttt gccaaatgaa gtgcctggta catcagatga 541 cagtactgaa gagccagtaa tgaaaaaacg tacttactgt acttactgcc cctctaaaat 601 aaggcgaaag gcaaatgcat cgtgcaaaaa atgcaaaaaa gttatttgtc gagagcataa 661 tattgatatg tgccaaagtt gtttctgact gactaataag tataatttgt ttctattatg 721 tataagttaa gctaattact tattttataa tacaacatga ctgtttttaa agtacaaaat 781 aagtttattt ttgtaaaaga gagaatgttt aaaagttttg ttactttata gaagaaattt 841 tgagtttttg ttttttttta ataaataaat aaacataaat aaattgtttg ttgaatttat 901 tattagtatg taagtgtaaa tataataaaa cttaatatct attcaaatta ataaataaac 961 ctcgatatac agaccgataa aacacatgcg tcaattttac gcatgattat ctttaacgta 1021 cgtcacaata tgattatctt tctagggtta aataatagtt tctaattttt ttattattca 1081 gcctgctgtc gtgaataccg tatatctcaa cgctgtctgt gagattgtcg tattctagcc $1141 \mathrm{tttttagttt} \mathrm{ttcgctcatc} \mathrm{gacttgatat} \mathrm{tgtccgacac} \mathrm{attttcgtcg} \mathrm{atttgcgttt}$ 1201 tgatcaaaga cttgagcaga gacacgttaa tcaactgttc aaattgatcc atattaacga 1261 tatcaacccg atgcgtatat ggtgcgtaaa atatattttt taaccctctt atactttgca 1321 ctctgcgtta atacgcgttc gtgtacagac gtaatcatgt tttctttttt ggataaaact 1381 cctactgagt ttgacctcat attagaccct cacaagttgc aaaacgtggc attttttacc 1441 aatgaagaat ttaaagttat tttaaaaaat ttcatcacag atttaaagaa gaaccaaaaa 1501 ttaaattatt taatcgacca gttaatcaac gtgtacacag agcgcaaaaa acacgcagcc 1561 cgacgtgttg gctaaaatta ttaaatcaac ttgtgttata gtcacgattt gccgtccaac 1621 gtgttcctca aaaagttgaa gaccaacaag tttacggaca ctagttaatt atttgatttt 1681 gccccacttc attttgtggg atcacaattt tgttatattt taaacaaagc ttggcactgg 1741 ccgtcgtttt acaacgtcgt gactgggaaa accctggcgt tacccaactt aatcgccttg 1801 cagcacatcc ccctttcgcc agctggcgta atagcgaaga ggcccgcacc gatcgccctt 
1861 cccaacagtt gcgcagcctg aatggcgaat ggcgectgat gcggtatttt ctccttacgc 1921 atctgtgcgg tatttcacac cgcatatggt gcactctcag tacaatctgc tctgatgccg 1981 catagttaag ccagccccga cacccgccaa cacccgctga cgcgccctga cgggcttgtc 2041 tgctcccggc atccgcttac agacaagctg tgaccgtctc cgggagctgc atgtgtcaga 2101 ggttttcacc gtcatcaccg aaacgcgcga gacgaaaggg cctcgtgata cgcctatttt 2161 tataggttaa tgtcatgata ataatggttt cttagacgtc aggtggcact tttcggggaa 2221 atgtgcgcgg aacccctatt tgtttatttt tctaaataca ttcaaatatg tatccgctca 2281 tgagacaata accctgataa atgcttcaat aatattgaaa aaggaagagt atgagtattc 2341 aacatttccg tgtcgccctt attccctttt ttgcgggatt ttgccttcct gtttttgctc 2401 acccagaaac gctggtgaaa gtaaaagatg ctgaagatca gttgggtgca cgagtgggtt 2461 acatcgaact ggatctcaac agcggtaaga tccttgagag ttttcgcccc gaagaacgtt 2521 ttccaatgat gagcactttt aaagttctgc tatgtggcgc ggtattatcc cgtattgacg 2581 ccgggcaaga gcaactcggt cgccgcatac actattctca gaatgacttg gttgagtact 2641 caccagtcac agaaaagcat cttacggatg gcatgacagt aagagaatta tgcagtgctg 2701 ccataaccat gagtgataac actgcggcca acttacttct gacaacgatc ggaggaccga 2761 aggagctaac cgcttttttg cacaacatgg gggatcatgt aactcgcctt gatcgttggg 2821 aaccggagct gaatgaagcc ataccaaacg acgagcgtga caccacgatg cctgtagcaa 2881 tggcaacaac gttgcgcaaa ctattaactg gcgaactact tactctagct tcccggcaac 2941 aattaataga ctggatggag gcggataaag ttgcaggacc acttctgcgc tcggcccttc 3001 cggctggctg gtttattgct gataaatctg gagccggtga gcgtgggtct cgcggtatca 3061 ttgcagcact ggggccagat ggtaagccct cccgtatcgt agttatctac acgacgggga 3121 gtcaggcaac tatggatgaa cgaaatagac agatcgctga gataggtgcc tcactgatta 3181 agcattggta actgtcagac caagtttact catatatact ttagattgat ttaaaacttc 3241 atttttaatt taaaaggatc taggtgaaga tcctttttga taatctcatg accaaaatcc 3301 cttaacgtga gttttcgttc cactgagcgt cagaccccgt agaaaagatc aaaggatctt 3361 cttgagatcc tttttttctg cgcgtaatct gctgcttgca aacaaaaaaa ccaccgctac 3421 cagcggtggt ttgtttgccg gatcaagagc taccaactct ttttccgaag gtaactggct 3481 tcagcagagc gcagatacca aatactgtcc ttctagtgta gccgtagtta ggccaccact 3541 tcaagaactc tgtagcaccg cctacatacc tcgctctgct aatcctgtta ccagtggctg 3601 ctgccagtgg cgataagtcg tgtcttaccg ggttggactc aagacgatag ttaccggata 3661 aggcgcagcg gtcgggctga acggggggtt cgtgcacaca gcccagcttg gagcgaacga 3721 cctacaccga actgagatac ctacagcgtg agcattgaga aagcgccacg cttcccgaag 3781 ggagaaaggc ggacaggtat ccggtaagcg gcagggtcgg aacaggagag cgcacgaggg 3841 agcttccagg gggaaacgcc tggtatcttt atagtcctgt cgggtttcgc cacctctgac 3901 ttgagcgtcg atttttgtga tgctcgtcag gggggcggag cctatggaaa aacgccagca 3961 acgcggcctt tttacggttc ctggcctttt gctggccttt tgctcacatg ttctttcctg 4021 cgttatcccc tgattctgtg gataaccgta ttaccgcctt tgagtgagct gataccgctc 4081 gccgcagccg aacgaccgag cgcagcgagt cagtgagcga ggaagcggaa gagcgcccaa 4141 tacgcaaacc gcctctcccc gcgcgttggc cgattcatta atgcagctgg cacgacaggt 4201 ttcccgactg gaaagcgggc agtgagcgca acgcaattaa tgtgagttag ctcactcatt 4261 aggcacccca ggctttacac tttatgcttc cggctcgtat gttgtgtgga attgtgagcg 4321 gataacaatt tcacacagga aacagctatg accatgatta cgaattcgag ctcggtaccc 4381 ggggatcctc tagagtcgac gctcgcgcga cttggtttgc cattctttag cgcgcgtcgc 4441 gtcacacagc ttggccacaa tgtggttttt gtcaaacgaa gattctatga cgtgtttaaa 4501 gtttaggtcg agtaaagcgc aaatcttttt taaccctaga aagatagtct gcgtaaaatt 4561 gacgcatgca ttcttgaaat attgctctct ctttctaaat agcgcgaatc cgtcgctgtg 4621 catttaggac atctcagtcg ccgcttggag ctcccgtgag gcgtgcttgt caatgcggta 4681 agtgtcactg attttgaact ataacgaccg cgtgagtcaa aatgacgcat gattatcttt 4741 tacgtgactt ttaagattta actcatacga taattatatt gttatttcat gttctactta 4801 cgtgataact tattatatat atatttctt gttatagata tcgtgactaa tatataataa 4861 aatgggtagt tctttagacg atgagcatat cctctctgct cttctgcaaa gcgatgacga 4921 gcttgttggt gaggattctg acagtgaaat atcagatcac gtaagtgaag atgacgtcca 4981 gagcgataca gaagaagcgt ttatagatga ggtacatgaa gtgcagccaa cgtcaagcgg 5041 tagtgaaata ttagacgaac aaaatgttat tgaacaacca ggttcttcat tggcttctaa 5101 cagaatcttg accttgccac agaggactat tagaggtaag aataaacatt gttggtcaac 5161 ttcaaagtcc acgaggcgta gccgagtctc tgcactgaac attgtcagat cttgtcaaac 5221 cacaaacata aaaaatagat gacagaaatt aacaaaatta ccaaaaaaac tagagaattt 5281 atttatttat ttttgttttt aatttatttt tgcctatggt cttacattgt tttacgcaac 5341 agttgcataa attattcggt ccaagatctt tccccaagat aatattttac atttggcgca 5401 acttgtgtag aattacattg cgtttatttt ctcaattacg gcgaaaatat acgacaaaat 5461 tgagatattt tcaattattc gtatgcaaaa ttgtctggag aattgattgg cgtcgtaaat 5521 tcgtaatagt ttttcaagtt ataaaccgaa atagattttg gctatttttg catttgttag 5581 caataataat atttttggaa actaccagta gcaggacaat gacctttttt taaacgatag 
5641 atgattaaaa agagttctga acacataaaa gttcataaaa gttgttggtt tggttggagc 5701 tactcagata aaaaaaaaat aattttttga aggtaaaaaa actgaaaaaa gaacagtctt 5761 ttcattacgt tatttacccg atatgcaaga aagttttctc tccaaaaatt attcaaataa 5821 gccgccttgt aagcgtcctc tatcacatcc cttcccaatt aggtcgactt ccgctcgcca 5881 cacgtcaaac cttttccatt tagaacgtca aaggccggcc CTAAAGAAAA ATGGCTGGTG 5941 GCAAAGCAGG GAAAGATTCG GGCAAAGCTA AAGCCAAGGC GGTGTCGCGC TCGGCGCGTG 6001 CCGGACTCCA GTTCCCCGTG GGGAGGATCC ACCGCCACTT GAAGAATCGC ACTACGAGCC 6061 ATGGACGCGT GGGGGCCACC GCGGCTGTGT ATTCCGCCGC GATTTTGGAG TATTTGACAG 6121 CTGAGGTCCT GGAGTTGGCC GGGAACGCCA GTAAGGACTT GAAGGTGAAG AGGATCACCC 6181 CCAGGCACTT GCAGCTGGCC ATCAGAGGGG ATGAGGAGCT GGACAGCCTG ATCAAGGCCA 6241 CTATCGCCGG CGGAGGCGTC ATTCCACACA TCCACAAGAG TTTGATCGGG AAAAAGGGCC 6301 ACTCACAGCC CGTCATGGTG AGCAAGGGCG AGGAGCTGTT CACCGGGGTG GTGCCCATCC 6361 TGGTCGAGCT GGACGGCGAC GTAAACGGCC ACAAGTTCAG CGTGTCCGGC GAGGGCGAGG 6421 GCGATGCCAC CTACGGCAAG CTGACCCTGA AGTTCATCTG CACCACCGGC AAGCTGCCCG 6481 TGCCCTGGCC CACCCTCGTG ACCACCCTGA CCTACGGCGT GCAGTGCTTC AGCCGCTACC 6541 CCGACCACAT GAAGCAGCAC GACTTCTTCA AGTCCGCCAT GCCCGAAGGC TACGTCCAGG 6601 AGCGCACCAT CTTCTTCAAG GACGACGGCA ACTACAAGAC CCGCGCCGAG GTGAAGTTCG 6661 AGGGCGACAC CCTGGTGAAC CGCATCGAGC TGAAGGGCAT CGACTTCAAG GAGGACGGCA 6721 ACATCCTGGG GCACAAGCTG GAGTACAACT ACAACAGCCA CAACGTCTAT ATCATGGCCG 6781 ACAAGCAGAA GAACGGCATC AAGGTGAACT TCAAGATCCG CCACAACATC GAGGACGGCA 6841 GCGTGCAGCT CGCCGACCAC TACCAGCAGA ACACCCCCAT CGGCGACGGC CCCGTGCTGC 6901 TGCCCGACAA CCACTACCTG AGCACCCAGT CCGCCCTGAG CAAAGACCCC AACGAGAAGC 6961 GCGATCACAT GGTCCTGCTG GAGTTCGTGA CCGCCGCCGG GATCACTCTC GGCATGGACG 7021 AGCTGTACAA GTAAggcgcg ccgtacgcga tcgtaagctt gatgagtttg gacaaaccac 7081 aactagaatg cagtgaaaaa aatgctttat ttgtgaaatt tgtgatgcta ttgctttatt 7141 tgtaaccatt ataagctgca ataaacaagt taacaacaac aattgcattc attttatgtt 7201 tcaggttcag ggggaagtgt gggaggtttt ttaaagcaag taaaacctct acaaatgtgg 7261 tatggctgat tatgatctag agtcgcggcc gccttaacga aataattaat tgtttccatt 7321 cgacacgata ttggtgctat ttgccgaccc ccaattgcac aaatgacttc tcatagacgt 7381 agacaagggg gggatgtagg accgtggtat caaaaacgtc gataaattga aaaggtccac 7441 gaaaacttta taccgatcgc tgaaaaacaa aatcaaacat tgcaaaaacc gtgttgaaat 7501 caatttcacc ttaacgttga ccttaaatac tgataccccg aagagccccc tgttcctaac 7561 tgcgaagaac caatcattct ttgcaccatc aaaacatgat tatctgaaat tataaaattt 7621 aaacaaaaac aattgacgta cggatttact aacaccgcca tttcgtgatc agcgagtcta 7681 tgtccatcaa aagtgttaga atttggtggg gctgactgaa cctgggttcg tccctgtaaa 7741 aggtgatcat gatagcccct tgcagggctt tgtgtgagaa gcgcctctcg ccgcgcgaca 7801 ttagagcctc atggacctca actttaaaaa tcgactcgta aacctcgcgt ctcttctcca 7861 aatcgttcaa tttgtaacgc tttaaagtct ccgcctcctc cttctccgcc agctcttttt 7921 gctcggttaa aagcttctcg accgcttttt ggtacttccc ccagaaatta aacccctcga 7981 gctccagccc cggcgttcgc tccagccacc gctggaccaa atcggtcaaa gacggctctt 8041 tctccgactt ggcgatttgc tcgagcgctt tttcgtcatt tccgaacact ttcgagtagt 8101 tctggttgta tttgacgcgg ttttcctggc gcaccccgag cttgttttcc aggagcctga 8161 actgcaggct ctggaacccg gaagcgggcc gcagataaca ccggaagtcc atgaaatcga 8221 gcggtgtcat cgtttccaga atcatcactt ggtccaccaa gacctacaat tgcgacagtt 8281 ttatctcgtg accgcaattt gttgcgaaat tgtgaacctt gaggatcaaa acaacacgat 8341 tgaggcgttt gaggatttcc aaagtttgcg actcttctaa aacgtcgctg aaaatgttgc 8401 ggatcgagtc cagctcgtag attatttgtt tgaaccataa ctcatacgct ggaattactt 8461 gttatagtta attacggtta agtgggtttt ttaccttgat gggtcacaat aaataaatgc 8521 tcgtcgtgca cgggctggtt attttgctcg ctgagtaatc tttgggcttc taaaatcttg 8581 tccaacatca agtattcacc gtaaagcatc ccacattctt cgctcagttg gtcgccttct 8641 tgggcttcac ttaatttacg attaattgta aatggaataa ttaaattaaa tcatacttac 8701 gagggtctca gtgggcaact cattacgacc gcggtaccgt cgacgttcta gcggtacccc 8761 gattgtttag cttgttcagc tgcgcttgtt tatttgctta gctttcgctt agcgacgtgt 8821 tcactttgct tgtttgaatt gaattgtcgc tccgtagacg aagcgcctct atttatactc 8881 cggcggtcga gggttcgaaa tcgataagct tggatcctaa ttgaattagc tctaattgaa 8941 ttagtctcta attgaattag atccccgggc gagctcgaat taattcaac 


\section{P3 9206...9196 \\ P3 9190...9180 \\ P3 9175...9165 \\ 3XP3ITATA $9206 \ldots 9118$ \\ TATA $9139 \ldots 9118$}

Tc'vermilionlgenomic $8979 \ldots 7541$

pBacR 21...1050

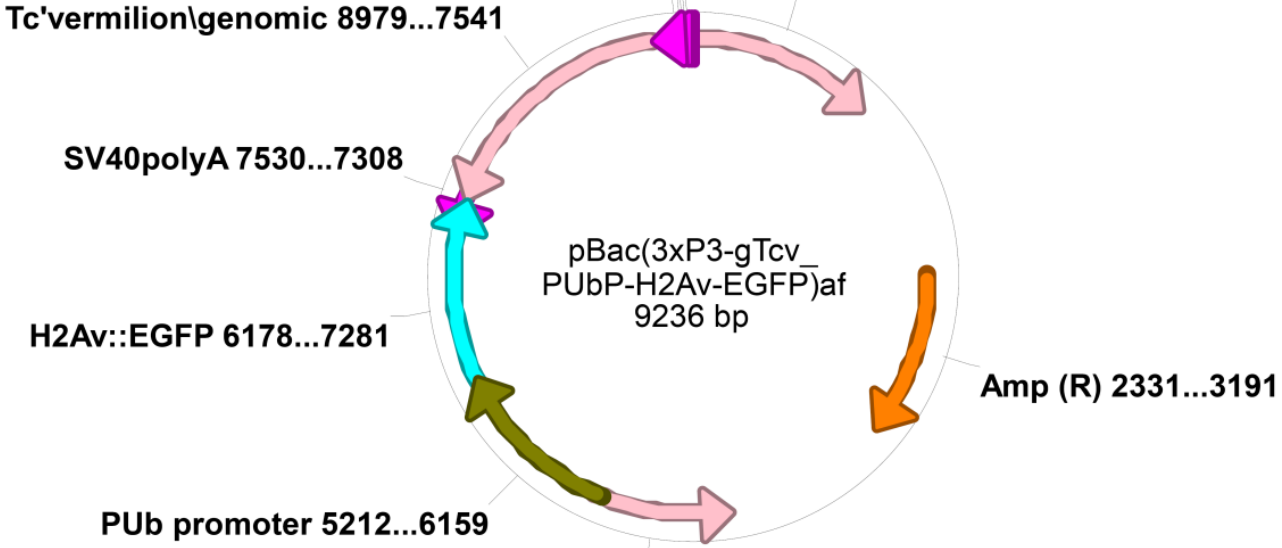

pBacL 5206...4489

Figure S7.19 pB[3xP3-gTc'v;Tc'PUbP- Tc'H2Av::EGFP].

Sequence S7.20 pB3[3xP3-gTc'v;Tc'PUbP- Tc'H2Av::EGFP], GenBank formatted.

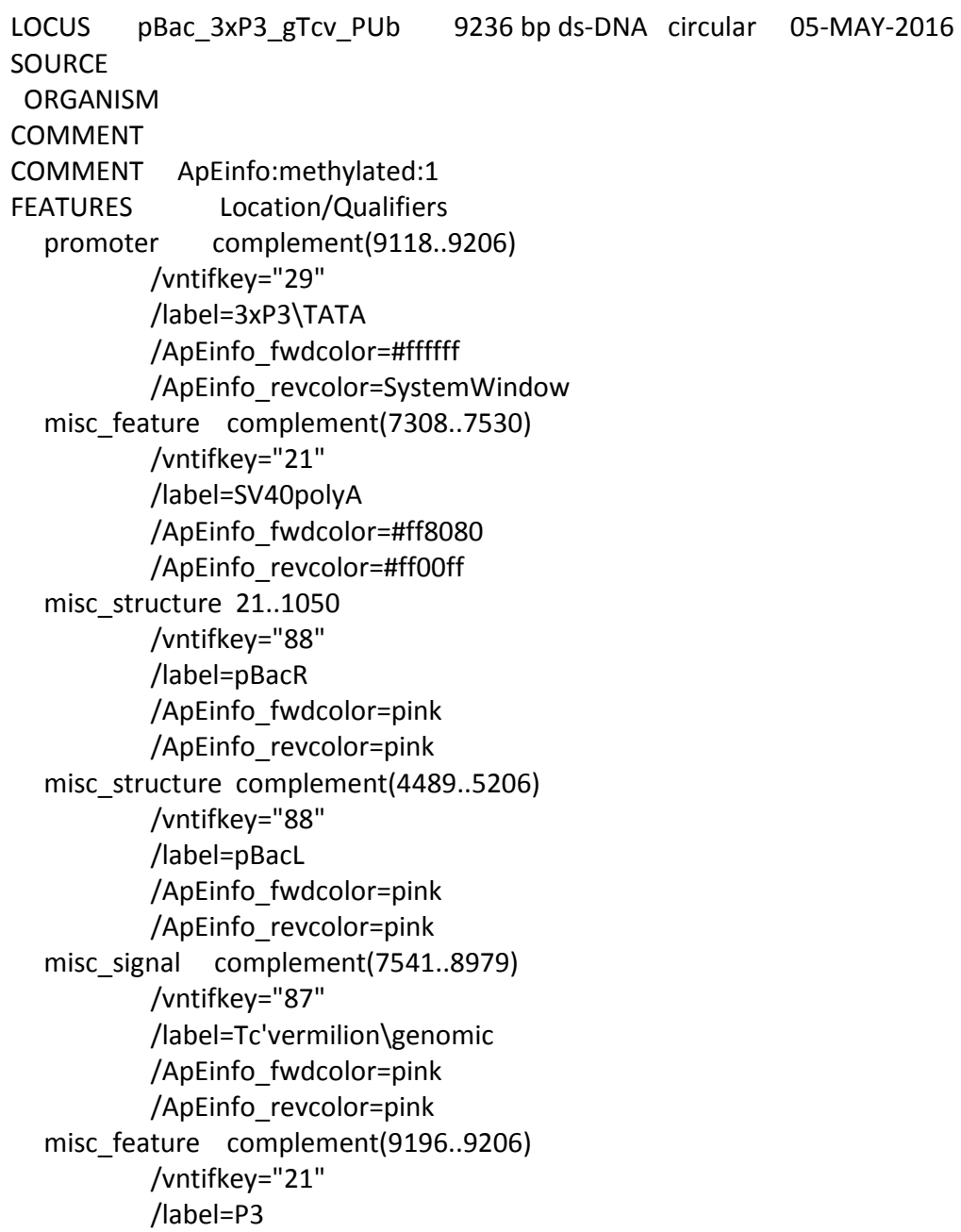


/ApEinfo_fwdcolor=\#ff8080

/ApEinfo_revcolor=\#ffOOff

misc_feature complement(9180..9190)

/vntifkey="21"

/label=P3(1)

/ApEinfo_label=P3

/ApEinfo_fwdcolor=\#ff8080

/ApEinfo_revcolor=\#ffOOff

misc_feature complement(9165..9175)

/vntifkey="21"

/label=P3(2)

/ApEinfo_label=P3

/ApEinfo_fwdcolor=\#ff8080

/ApEinfo_revcolor=\#ffoOff

misc_feature complement(9118..9139)

/vntifkey="21"

/label=TATA

/ApEinfo_fwdcolor=\#ff8080

/ApEinfo_revcolor=\#ffooff

misc_feature 6178..7281

/label=H2Av::EGFP

/ApEinfo_fwdcolor=cyan

/ApEinfo_revcolor=green

misc feature 5212..6159

/label=PUb promoter

/ApEinfo_fwdcolor $=\# 808000$

/ApEinfo_revcolor=green

misc_feature 2331..3191

/label=Amp (R)

/ApEinfo_fwdcolor=\#ff8000

/ApEinfo_revcolor=green

ORIGIN

1 cattgtggga accgtgcgat caaacaaacg cgagataccg gaagtactga aaaacagtcg 61 ctccaggcca gtgggaacat cgatgttttg ttttgacgga ccccttactc tcgtctcata 121 taaaccgaag ccagctaaga tggtatactt attatcatct tgtgatgagg atgcttctat 181 caacgaaagt accggtaaac cgcaaatggt tatgtattat aatcaaacta aaggcggagt 241 ggacacgcta gaccaaatgt gttctgtgat gacctgcagt aggaagacga ataggtggcc 301 tatggcatta ttgtacggaa tgataaacat tgcctgcata aattcttta ttatatacag 361 ccataatgtc agtagcaagg gagaaaaggt tcaaagtcgc aaaaaattta tgagaaacct 421 ttacatgagc ctgacgtcat cgtttatgcg taagcgttta gaagctccta ctttgaagag 481 atatttgcgc gataatatct ctaatatttt gccaaatgaa gtgcctggta catcagatga 541 cagtactgaa gagccagtaa tgaaaaaacg tacttactgt acttactgcc cctctaaaat 601 aaggcgaaag gcaaatgcat cgtgcaaaaa atgcaaaaaa gttatttgtc gagagcataa 661 tattgatatg tgccaaagtt gtttctgact gactaataag tataatttgt ttctattatg 721 tataagttaa gctaattact tattttataa tacaacatga ctgtttttaa agtacaaaat 781 aagtttattt ttgtaaaaga gagaatgttt aaaagttttg ttactttata gaagaaattt 841 tgagtttttg ttttttttta ataaataaat aaacataaat aaattgtttg ttgaatttat 901 tattagtatg taagtgtaaa tataataaaa cttaatatct attcaaatta ataaataaac 961 ctcgatatac agaccgataa aacacatgcg tcaattttac gcatgattat ctttaacgta 1021 cgtcacaata tgattatctt tctagggtta aataatagtt tctaattttt ttattattca 1081 gcctgctgtc gtgaataccg tatatctcaa cgctgtctgt gagattgtcg tattctagcc $1141 \mathrm{tttttagttt} \mathrm{ttcgctcatc} \mathrm{gacttgatat} \mathrm{tgtccgacac} \mathrm{attttcgtcg} \mathrm{atttgcgttt}$ 1201 tgatcaaaga cttgagcaga gacacgttaa tcaactgttc aaattgatcc atattaacga 1261 tatcaacccg atgcgtatat ggtgcgtaaa atatattttt taaccctctt atactttgca 1321 ctctgcgtta atacgcgttc gtgtacagac gtaatcatgt tttctttttt ggataaaact 1381 cctactgagt ttgacctcat attagaccct cacaagttgc aaaacgtggc attttttacc 1441 aatgaagaat ttaaagttat tttaaaaaat ttcatcacag atttaaagaa gaaccaaaaa 1501 ttaaattatt taatcgacca gttaatcaac gtgtacacag agcgcaaaaa acacgcagcc 1561 cgacgtgttg gctaaaatta ttaaatcaac ttgtgttata gtcacgattt gccgtccaac 1621 gtgttcctca aaaagttgaa gaccaacaag tttacggaca ctagttaatt atttgatttt 1681 gccccacttc attttgtggg atcacaattt tgttatattt taaacaaagc ttggcactgg 1741 ccgtcgtttt acaacgtcgt gactgggaaa accctggcgt tacccaactt aatcgccttg 1801 cagcacatcc ccctttcgcc agctggcgta atagcgaaga ggcccgcacc gatcgecctt 
1861 cccaacagtt gcgcagcctg aatggcgaat ggcgectgat gcggtatttt ctccttacgc 1921 atctgtgcgg tatttcacac cgcatatggt gcactctcag tacaatctgc tctgatgccg 1981 catagttaag ccagccccga cacccgccaa cacccgctga cgcgccctga cgggcttgtc 2041 tgctcccggc atccgcttac agacaagctg tgaccgtctc cgggagctgc atgtgtcaga 2101 ggttttcacc gtcatcaccg aaacgcgcga gacgaaaggg cctcgtgata cgcctatttt 2161 tataggttaa tgtcatgata ataatggttt cttagacgtc aggtggcact tttcggggaa 2221 atgtgcgcgg aacccctatt tgtttatttt tctaaataca ttcaaatatg tatccgctca 2281 tgagacaata accctgataa atgcttcaat aatattgaaa aaggaagagt atgagtattc 2341 aacatttccg tgtcgccctt attccctttt ttgcggeatt ttgccttcct gtttttgctc 2401 acccagaaac gctggtgaaa gtaaaagatg ctgaagatca gttgggtgca cgagtgggtt 2461 acatcgaact ggatctcaac agcggtaaga tccttgagag ttttcgcccc gaagaacgtt 2521 ttccaatgat gagcactttt aaagttctgc tatgtggcgc ggtattatcc cgtattgacg 2581 ccgggcaaga gcaactcggt cgccgcatac actattctca gaatgacttg gttgagtact 2641 caccagtcac agaaaagcat cttacggatg gcatgacagt aagagaatta tgcagtgctg 2701 ccataaccat gagtgataac actgcggcca acttacttct gacaacgatc ggaggaccga 2761 aggagctaac cgcttttttg cacaacatgg gggatcatgt aactcgcctt gatcgttggg 2821 aaccggagct gaatgaagcc ataccaaacg acgagcgtga caccacgatg cctgtagcaa 2881 tggcaacaac gttgcgcaaa ctattaactg gcgaactact tactctagct tcccggcaac 2941 aattaataga ctggatggag gcggataaag ttgcaggacc acttctgcgc tcggcccttc 3001 cggctggctg gtttattgct gataaatctg gagccggtga gcgtgggtct cgcggtatca 3061 ttgcagcact ggggccagat ggtaagccct cccgtatcgt agttatctac acgacgggga 3121 gtcaggcaac tatggatgaa cgaaatagac agatcgctga gataggtgcc tcactgatta 3181 agcattggta actgtcagac caagtttact catatatact ttagattgat ttaaaacttc 3241 atttttaatt taaaaggatc taggtgaaga tcctttttga taatctcatg accaaaatcc 3301 cttaacgtga gttttcgttc cactgagcgt cagaccccgt agaaaagatc aaaggatctt 3361 cttgagatcc tttttttctg cgcgtaatct gctgcttgca aacaaaaaaa ccaccgctac 3421 cagcggtggt ttgtttgccg gatcaagagc taccaactct ttttccgaag gtaactggct 3481 tcagcagagc gcagatacca aatactgtcc ttctagtgta gccgtagtta ggccaccact 3541 tcaagaactc tgtagcaccg cctacatacc tcgctctgct aatcctgtta ccagtggctg 3601 ctgccagtgg cgataagtcg tgtcttaccg ggttggactc aagacgatag ttaccggata 3661 aggcgcagcg gtcgggctga acggggggtt cgtgcacaca gcccagcttg gagcgaacga 3721 cctacaccga actgagatac ctacagcgtg agcattgaga aagcgccacg cttcccgaag 3781 ggagaaaggc ggacaggtat ccggtaagcg gcagggtcgg aacaggagag cgcacgaggg 3841 agcttccagg gggaaacgcc tggtatcttt atagtcctgt cgggtttcgc cacctctgac 3901 ttgagcgtcg atttttgtga tgctcgtcag gggggcggag cctatggaaa aacgccagca 3961 acgcggcctt tttacggttc ctggcctttt gctggccttt tgctcacatg ttctttcctg 4021 cgttatcccc tgattctgtg gataaccgta ttaccgcctt tgagtgagct gataccgctc 4081 gccgcagccg aacgaccgag cgcagcgagt cagtgagcga ggaagcggaa gagcgcccaa 4141 tacgcaaacc gcctctcccc gcgcgttggc cgattcatta atgcagctgg cacgacaggt 4201 ttcccgactg gaaagcgggc agtgagcgca acgcaattaa tgtgagttag ctcactcatt 4261 aggcacccca ggctttacac tttatgcttc cggctcgtat gttgtgtgga attgtgagcg 4321 gataacaatt tcacacagga aacagctatg accatgatta cgaattcgag ctcggtaccc 4381 ggggatcctc tagagtcgac gctcgcgcga cttggtttgc cattctttag cgcgcgtcgc 4441 gtcacacagc ttggccacaa tgtggttttt gtcaaacgaa gattctatga cgtgtttaaa 4501 gtttaggtcg agtaaagcgc aaatcttttt taaccctaga aagatagtct gcgtaaaatt 4561 gacgcatgca ttcttgaaat attgctctct ctttctaaat agcgcgaatc cgtcgctgtg 4621 catttaggac atctcagtcg ccgcttggag ctcccgtgag gcgtgcttgt caatgcggta 4681 agtgtcactg attttgaact ataacgaccg cgtgagtcaa aatgacgcat gattatcttt 4741 tacgtgactt ttaagattta actcatacga taattatatt gttatttcat gttctactta 4801 cgtgataact tattatatat atattttctt gttatagata tcgtgactaa tatataataa 4861 aatgggtagt tctttagacg atgagcatat cctctctgct cttctgcaaa gcgatgacga 4921 gcttgttggt gaggattctg acagtgaaat atcagatcac gtaagtgaag atgacgtcca 4981 gagcgataca gaagaagcgt ttatagatga ggtacatgaa gtgcagccaa cgtcaagcgg 5041 tagtgaaata ttagacgaac aaaatgttat tgaacaacca ggttcttcat tggcttctaa 5101 cagaatcttg accttgccac agaggactat tagaggtaag aataaacatt gttggtcaac 5161 ttcaaagtcc acgaggcgta gccgagtctc tgcactgaac attgtcagat cTGTACTTTT 5221 CTTTGTCCCA AATGACCCTT TTACATAATT TATTATTGTC GTCCGTATTT ACATGATAAA 5281 CAAGACTCTA GAACAACAAC GATAAGGATA ATTCTTTGTA AACAAATAAA TTCCTTTCAA 5341 AATTGAACAG ACGAATTTGG CATTTCATGC AGGTATTAAT GGGTGGTTTA TTTGAAACGT 5401 TTGAAAAGCA TAAATTGGAA AATAAATTAA AATTTGAAAA ATAAAAAAGG GCGTAATTCC 5461 AAAACAAAGA AAACAGCTGA TCGATAAAGC TGACGACGTA GATAAAAATC AGCTGACATG 5521 TCACCAACAA CTACTGGCCA AAGTCCCTAG AATCTTGTTC AATTAAAAGC AATTTTCTTA 5581 ATACCTGAAA CATTGTTTTG ACCACGATTA ACCGAATTGG CATTAAACTG ATAGCAACTA 
5641 AAGTTTATGT ATAAATTTTT GATTAAAGAG GACGTTTTTT GGATAATTAG TCGAAAATTC 5701 TAGAAAGTGT ACTTTGGGTT CGAGATTAAA AAGGAGTGGG GGAAGCCGTC CGTATAAAAG 5761 CGGTTCCACA GCTGCATTCG CCATATTGTC TGAGCAAAAG TGGTGAAATA CAAAAGCGAT 5821 TTAATTAAAA TTGTGAATTT TCCTAGCCGT AAAGGTATGT ATTTTAATTT CATCATGAAT 5881 TATTCGATTG CAAGAAGCGC AGAAAGTCGC AATTTTGTGA AATTTTTCGC ATGACGCAAT 5941 AACATTTGTT TGAAAAAACA TCGCCCTAGT GACGTGTTTT TTTGTTTAAA TTTTAAACAAA 6001 ATTAATTTTG TAAATTTGTC GATCTGTTCA AAACCAAACC ACATAGTGAG TAATGTGTTT 6061 TTTCGGTTTA TTCAGTGCGT AATTCTTTGT TCAACGAAAT CCAATATGGC GGCTTGGAAA 6121 TTTGCTTTGT CACAAAAGTA ATTTTTTGTG TCGTTGCAGg gccggccCTA AAGAAAAATG 6181 GCTGGTGGCA AAGCAGGGAA AGATTCGGGC AAAGCTAAAG CCAAGGCGGT GTCGCGCTCG 6241 GCGCGTGCCG GACTCCAGTT CCCCGTGGGG AGGATCCACC GCCACTTGAA GAATCGCACT 6301 ACGAGCCATG GACGCGTGGG GGCCACCGCG GCTGTGTATT CCGCCGCGAT TTTGGAGTAT 6361 TTGACAGCTG AGGTCCTGGA GTTGGCCGGG AACGCCAGTA AGGACTTGAA GGTGAAGAGG 6421 ATCACCCCCA GGCACTTGCA GCTGGCCATC AGAGGGGATG AGGAGCTGGA CAGCCTGATC 6481 AAGGCCACTA TCGCCGGCGG AGGCGTCATT CCACACATCC ACAAGAGTTT GATCGGGAAA 6541 AAGGGCCACT CACAGCCCGT CATGGTGAGC AAGGGCGAGG AGCTGTTCAC CGGGGTGGTG 6601 CCCATCCTGG TCGAGCTGGA CGGCGACGTA AACGGCCACA AGTTCAGCGT GTCCGGCGAG 6661 GGCGAGGGCG ATGCCACCTA CGGCAAGCTG ACCCTGAAGT TCATCTGCAC CACCGGCAAG 6721 CTGCCCGTGC CCTGGCCCAC CCTCGTGACC ACCCTGACCT ACGGCGTGCA GTGCTTCAGC 6781 CGCTACCCCG ACCACATGAA GCAGCACGAC TTCTTCAAGT CCGCCATGCC CGAAGGCTAC 6841 GTCCAGGAGC GCACCATCTT CTTCAAGGAC GACGGCAACT ACAAGACCCG CGCCGAGGTG 6901 AAGTTCGAGG GCGACACCCT GGTGAACCGC ATCGAGCTGA AGGGCATCGA CTTCAAGGAG 6961 GACGGCAACA TCCTGGGGCA CAAGCTGGAG TACAACTACA ACAGCCACAA CGTCTATATC 7021 ATGGCCGACA AGCAGAAGAA CGGCATCAAG GTGAACTTCA AGATCCGCCA CAACATCGAG 7081 GACGGCAGCG TGCAGCTCGC CGACCACTAC CAGCAGAACA CCCCCATCGG CGACGGCCCC 7141 GTGCTGCTGC CCGACAACCA CTACCTGAGC ACCCAGTCCG CCCTGAGCAA AGACCCCAAC 7201 GAGAAGCGCG ATCACATGGT CCTGCTGGAG TTCGTGACCG CCGCCGGGAT CACTCTCGGC 7261 ATGGACGAGC TGTACAAGTA Aggcgcgccg tacgcgatcg taagcttgat gagtttggac 7321 aaaccacaac tagaatgcag tgaaaaaaat gctttatttg tgaaatttgt gatgctattg 7381 ctttatttgt aaccattata agctgcaata aacaagttaa caacaacaat tgcattcatt 7441 ttatgtttca ggttcagggg gaagtgtggg aggtttttta aagcaagtaa aacctctaca 7501 aatgtggtat ggctgattat gatctagagt cgcggccgcc ttaacgaaat aattaattgt 7561 ttccattcga cacgatattg gtgctatttg ccgaccccca attgcacaaa tgacttctca 7621 tagacgtaga caaggggggg atgtaggacc gtggtatcaa aaacgtcgat aaattgaaaa 7681 ggtccacgaa aactttatac cgatcgctga aaaacaaaat caaacattgc aaaaaccgtg 7741 ttgaaatcaa tttcacctta acgttgacct taaatactga taccccgaag agccccctgt 7801 tcctaactgc gaagaaccaa tcattctttg caccatcaaa acatgattat ctgaaattat 7861 aaaatttaaa caaaaacaat tgacgtacgg atttactaac accgccattt cgtgatcagc 7921 gagtctatgt ccatcaaaag tgttagaatt tggtggggct gactgaacct gggttcgtcc 7981 ctgtaaaagg tgatcatgat agccccttgc agggctttgt gtgagaagcg cctctcgccg 8041 cgcgacatta gagcctcatg gacctcaact ttaaaaatcg actcgtaaac ctcgcgtctc 8101 ttctccaaat cgttcaattt gtaacgcttt aaagtctccg cctcctcctt ctccgccagc 8161 tctttttgct cggttaaaag cttctcgacc gctttttggt acttccccca gaaattaaac 8221 ccctcgagct ccagccccgg cgttcgctcc agccaccgct ggaccaaatc ggtcaaagac 8281 ggctctttct ccgacttggc gatttgctcg agcgcttttt cgtcatttcc gaacactttc 8341 gagtagttct ggttgtattt gacgcggttt tcctggcgca ccccgagctt gttttccagg 8401 agcctgaact gcaggctctg gaacccggaa gcgggccgca gataacaccg gaagtccatg 8461 aaatcgagcg gtgtcatcgt ttccagaatc atcacttggt ccaccaagac ctacaattgc 8521 gacagtttta tctcgtgacc gcaatttgtt gcgaaattgt gaaccttgag gatcaaaaca 8581 acacgattga ggcgtttgag gatttccaaa gtttgcgact cttctaaaac gtcgctgaaa 8641 atgttgcgga tcgagtccag ctcgtagatt atttgtttga accataactc atacgctgga 8701 attacttgtt atagttaatt acggttaagt gggtttttta ccttgatggg tcacaataaa 8761 taaatgctcg tcgtgcacgg gctggttatt ttgctcgctg agtaatcttt gggcttctaa 8821 aatcttgtcc aacatcaagt attcaccgta aagcatccca cattcttcgc tcagttggtc 8881 gccttcttgg gcttcactta atttacgatt aattgtaaat ggaataatta aattaaatca 8941 tacttacgag ggtctcagtg ggcaactcat tacgaccgcg gtaccgtcga cgttctagcg 9001 gtaccccgat tgtttagctt gttcagctgc gcttgtttat ttgcttagct ttcgcttagc 9061 gacgtgttca ctttgcttgt ttgaattgaa ttgtcgctcc gtagacgaag cgcctctatt 9121 tatactccgg cggtcgaggg ttcgaaatcg ataagcttgg atcctaattg aattagctct 9181 aattgaatta gtctctaatt gaattagatc cccgggcgag ctcgaattaa ttcaac 
P3 8311...8301

P3 8295...8285

P3 8280...8270

3xP3ITATA $8311 \ldots 8223$

TATA $8244 \ldots 8223$

Tc'vermilionlgenomic 8084...6646

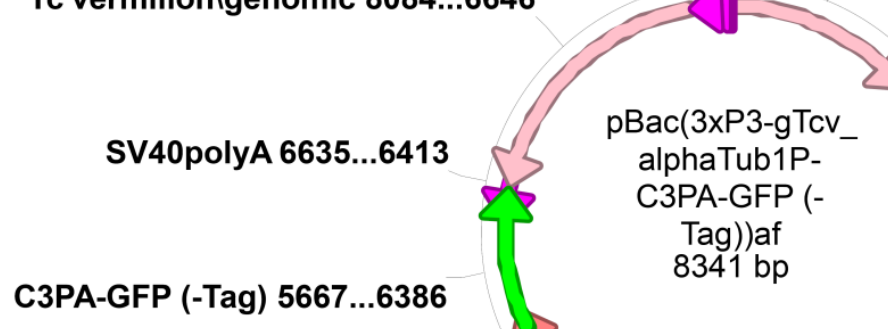

pBacR 21...1050

alphaTub1 promoter $5213 \ldots 5648$

pBacL 5206...4489

Figure S7.21 pB[3xP3-gTc'v;Tc' $\alpha$ Tub1P-C3PA-GFP].

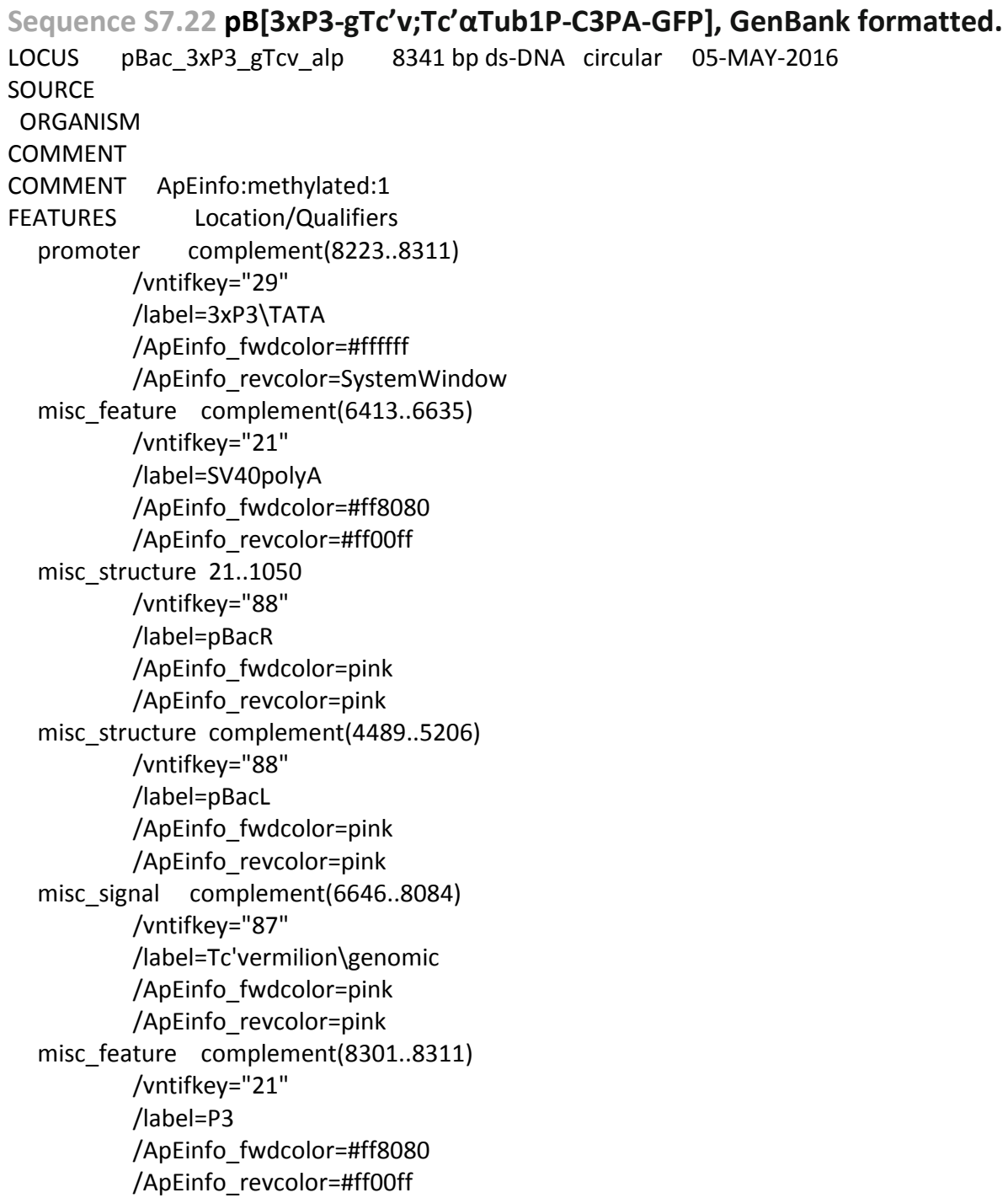


misc_feature complement(8285..8295)

/vntifkey="21"

/label=P3(1)

/ApEinfo_label=P3

/ApEinfo_fwdcolor=\#ff8080

/ApEinfo_revcolor=\#ffOOff

misc_feature complement(8270..8280)

/vntifkey="21"

/label=P3(2)

/ApEinfo_label=P3

/ApEinfo_fwdcolor=\#ff8080

/ApEinfo_revcolor=\#ffOOff

misc_feature complement(8223..8244)

/vntifkey="21"

/label=TATA

/ApEinfo_fwdcolor=\#ff8080

/ApEinfo_revcolor=\#ffOOff

misc_feature 5213..5648

/vntifkey="21"

/label=alphaTub1 promoter

/ApEinfo_fwdcolor=\#ff8080

/ApEinfo_revcolor=\#ffOOff

misc_feature 5667..6386

/label=C3PA-GFP (-Tag)

/ApEinfo_fwdcolor=\#00ffO0

/ApEinfo_revcolor=green

misc feature 2331..3191

/label=Amp (R)

/ApEinfo_fwdcolor=\#ff8000

/ApEinfo_revcolor=green

ORIGIN

1 cattgtggga accgtgcgat caaacaaacg cgagataccg gaagtactga aaaacagtcg

61 ctccaggcca gtgggaacat cgatgttttg ttttgacgga ccccttactc tcgtctcata

121 taaaccgaag ccagctaaga tggtatactt attatcatct tgtgatgagg atgcttctat

181 caacgaaagt accggtaaac cgcaaatggt tatgtattat aatcaaacta aaggcggagt

241 ggacacgcta gaccaaatgt gttctgtgat gacctgcagt aggaagacga ataggtggcc

301 tatggcatta ttgtacggaa tgataaacat tgcctgcata aattctttta ttatatacag

361 ccataatgtc agtagcaagg gagaaaaggt tcaaagtcgc aaaaaattta tgagaaacct

421 ttacatgagc ctgacgtcat cgtttatgcg taagcgttta gaagctccta ctttgaagag

481 atatttgcgc gataatatct ctaatatttt gccaaatgaa gtgcctggta catcagatga

541 cagtactgaa gagccagtaa tgaaaaaacg tacttactgt acttactgcc cctctaaaat

601 aaggcgaaag gcaaatgcat cgtgcaaaaa atgcaaaaaa gttatttgtc gagagcataa 661 tattgatatg tgccaaagtt gtttctgact gactaataag tataatttgt ttctattatg

721 tataagttaa gctaattact tattttataa tacaacatga ctgtttttaa agtacaaaat 781 aagtttattt ttgtaaaaga gagaatgttt aaaagttttg ttactttata gaagaaattt 841 tgagtttttg ttttttttta ataaataaat aaacataaat aaattgtttg ttgaatttat 901 tattagtatg taagtgtaaa tataataaaa cttaatatct attcaaatta ataaataaac 961 ctcgatatac agaccgataa aacacatgcg tcaattttac gcatgattat ctttaacgta 1021 cgtcacaata tgattatctt tctagggtta aataatagtt tctaattttt ttattattca 1081 gcctgctgtc gtgaataccg tatatctcaa cgctgtctgt gagattgtcg tattctagcc $1141 \mathrm{tttttagttt} \mathrm{ttcgctcatc} \mathrm{gacttgatat} \mathrm{tgtccgacac} \mathrm{attttcgtcg} \mathrm{atttgcgttt}$ 1201 tgatcaaaga cttgagcaga gacacgttaa tcaactgttc aaattgatcc atattaacga 1261 tatcaacccg atgcgtatat ggtgcgtaaa atatattttt taaccctctt atactttgca 1321 ctctgcgtta atacgcgttc gtgtacagac gtaatcatgt tttctttttt ggataaaact 1381 cctactgagt ttgacctcat attagaccct cacaagttgc aaaacgtggc attttttacc 1441 aatgaagaat ttaaagttat tttaaaaaat ttcatcacag atttaaagaa gaaccaaaaa 1501 ttaaattatt taatcgacca gttaatcaac gtgtacacag agcgcaaaaa acacgcagcc 1561 cgacgtgttg gctaaaatta ttaaatcaac ttgtgttata gtcacgattt gccgtccaac 1621 gtgttcctca aaaagttgaa gaccaacaag tttacggaca ctagttaatt atttgatttt 1681 gccccacttc attttgtggg atcacaattt tgttatattt taaacaaagc ttggcactgg 1741 ccgtcgtttt acaacgtcgt gactgggaaa accctggcgt tacccaactt aatcgccttg 1801 cagcacatcc ccctttcgcc agctggcgta atagcgaaga ggcccgcacc gatcgccctt 1861 cccaacagtt gcgcagcctg aatggcgaat ggcgcctgat gcggtatttt ctccttacgc 
1921 atctgtgcgg tatttcacac cgcatatggt gcactctcag tacaatctgc tctgatgccg 1981 catagttaag ccagccccga cacccgccaa cacccgctga cgcgccctga cgggcttgtc 2041 tgctcccggc atccgcttac agacaagctg tgaccgtctc cgggagctgc atgtgtcaga 2101 ggttttcacc gtcatcaccg aaacgcgcga gacgaaaggg cctcgtgata cgcctatttt 2161 tataggttaa tgtcatgata ataatggttt cttagacgtc aggtggcact tttcggggaa 2221 atgtgcgcgg aacccctatt tgtttatttt tctaaataca ttcaaatatg tatccgctca 2281 tgagacaata accctgataa atgcttcaat aatattgaaa aaggaagagt atgagtattc 2341 aacatttccg tgtcgccctt attccctttt ttgcggcatt ttgccttcct gtttttgctc 2401 acccagaaac gctggtgaaa gtaaaagatg ctgaagatca gttgggtgca cgagtgggtt 2461 acatcgaact ggatctcaac agcggtaaga tccttgagag ttttcgcccc gaagaacgtt 2521 ttccaatgat gagcactttt aaagttctgc tatgtggcgc ggtattatcc cgtattgacg 2581 ccgggcaaga gcaactcggt cgccgcatac actattctca gaatgacttg gttgagtact 2641 caccagtcac agaaaagcat cttacggatg gcatgacagt aagagaatta tgcagtgctg 2701 ccataaccat gagtgataac actgcggcca acttacttct gacaacgatc ggaggaccga 2761 aggagctaac cgcttttttg cacaacatgg gggatcatgt aactcgcctt gatcgttggg 2821 aaccggagct gaatgaagcc ataccaaacg acgagcgtga caccacgatg cctgtagcaa 2881 tggcaacaac gttgcgcaaa ctattaactg gcgaactact tactctagct tcccggcaac 2941 aattaataga ctggatggag gcggataaag ttgcaggacc acttctgcgc tcggcccttc 3001 cggctggctg gtttattgct gataaatctg gagccggtga gcgtgggtct cgcggtatca 3061 ttgcagcact ggggccagat ggtaagccct cccgtatcgt agttatctac acgacgggga 3121 gtcaggcaac tatggatgaa cgaaatagac agatcgctga gataggtgcc tcactgatta 3181 agcattggta actgtcagac caagtttact catatatact ttagattgat ttaaaacttc 3241 atttttaatt taaaaggatc taggtgaaga tcctttttga taatctcatg accaaaatcc 3301 cttaacgtga gttttcgttc cactgagcgt cagaccccgt agaaaagatc aaaggatctt 3361 cttgagatcc tttttttctg cgcgtaatct gctgcttgca aacaaaaaaa ccaccgctac 3421 cagcggtggt ttgtttgccg gatcaagagc taccaactct ttttccgaag gtaactggct 3481 tcagcagagc gcagatacca aatactgtcc ttctagtgta gccgtagtta ggccaccact 3541 tcaagaactc tgtagcaccg cctacatacc tcgctctgct aatcctgtta ccagtggctg 3601 ctgccagtgg cgataagtcg tgtcttaccg ggttggactc aagacgatag ttaccggata 3661 aggcgcagcg gtcgggctga acggggggtt cgtgcacaca gcccagcttg gagcgaacga 3721 cctacaccga actgagatac ctacagcgtg agcattgaga aagcgccacg cttcccgaag 3781 ggagaaaggc ggacaggtat ccggtaagcg gcagggtcgg aacaggagag cgcacgaggg 3841 agcttccagg gggaaacgcc tggtatcttt atagtcctgt cgggtttcgc cacctctgac 3901 ttgagcgtcg atttttgtga tgctcgtcag gggggeggag cctatggaaa aacgccagca 3961 acgcggcctt tttacggttc ctggcctttt gctggccttt tgctcacatg ttctttcctg 4021 cgttatcccc tgattctgtg gataaccgta ttaccgcctt tgagtgagct gataccgctc 4081 gccgcagccg aacgaccgag cgcagcgagt cagtgagcga ggaagcggaa gagcgcccaa 4141 tacgcaaacc gcctctcccc gcgcgttggc cgattcatta atgcagctgg cacgacaggt 4201 ttcccgactg gaaagcgggc agtgagcgca acgcaattaa tgtgagttag ctcactcatt 4261 aggcacccca ggctttacac tttatgcttc cggctcgtat gttgtgtgga attgtgagcg 4321 gataacaatt tcacacagga aacagctatg accatgatta cgaattcgag ctcggtaccc 4381 ggggatcctc tagagtcgac gctcgcgcga cttggtttgc cattctttag cgcgcgtcgc 4441 gtcacacagc ttggccacaa tgtggttttt gtcaaacgaa gattctatga cgtgtttaaa 4501 gtttaggtcg agtaaagcgc aaatcttttt taaccctaga aagatagtct gcgtaaaatt 4561 gacgcatgca ttcttgaaat attgctctct ctttctaaat agcgcgaatc cgtcgctgtg 4621 catttaggac atctcagtcg ccgcttggag ctcccgtgag gcgtgcttgt caatgcggta 4681 agtgtcactg attttgaact ataacgaccg cgtgagtcaa aatgacgcat gattatcttt 4741 tacgtgactt ttaagattta actcatacga taattatatt gttatttcat gttctactta 4801 cgtgataact tattatatat atattttctt gttatagata tcgtgactaa tatataataa 4861 aatgggtagt tctttagacg atgagcatat cctctctgct cttctgcaaa gcgatgacga 4921 gcttgttggt gaggattctg acagtgaaat atcagatcac gtaagtgaag atgacgtcca 4981 gagcgataca gaagaagcgt ttatagatga ggtacatgaa gtgcagccaa cgtcaagcgg 5041 tagtgaaata ttagacgaac aaaatgttat tgaacaacca ggttcttcat tggcttctaa 5101 cagaatcttg accttgccac agaggactat tagaggtaag aataaacatt gttggtcaac 5161 ttcaaagtcc acgaggcgta gccgagtctc tgcactgaac attgtcagat ctcactgcag 5221 tgaacggtta tgatggtgca acgaccggga cctcagctgt ataaaaaatc tgaaaataat 5281 tgataatggt cgaagaccat aaggaaaaat aattcgaaaa ttaaaggcaa agtatgaaaa 5341 tgctggaaat aaatcgggaa acaccgaatt tttggtcaca ggaaccaacg aatttttttt 5401 tgaatattga tttgaaaaga gatcgagaac aaggataaca atcttccgtt agagaccgtt 5461 ggtaccgaca tggcggggag ccaatcagaa gtctggaaat cgggcgttgg ttgctgacgt 5521 caacaagctt ataaatagca acgacagttg aaaatcgaat caaagtcgtt tggaaaaagc 5581 cagagcttgt atttccgaag cgtactcccg tttttctgct cttttgtggt gtaatttgta 5641 aaactcaagg ccggccCTAA AGAAAAATGG TGAGCAAGGG CGAGGAGCTG TTCACCGGGG 
5701 TGGTGCCCAT CCTGGTCGAG CTGGACGGCG ACGTAAACGG CCACAAGTTC AGCGTGTCCG 5761 GCGAGGGCGA GGGCGATGCC ACCTACGGCA AGCTGACCCT GAAGTTCATC TGCACCACCG 5821 GCAAGCTGCC CGTGCCCTGG CCCACCCTCG TGACCACCTT CAGCTACGGC GTGCAGTGCT 5881 TCAGCCGCTA CCCCGACCAC ATGAAGCAGC ACGACTTCTT CAAGTCCGCC ATGCCCGAAG 5941 GCTACGTCCA GGAGCGCACC ATCTCCTTCA AGGACGACGG CAACTACAAG ACCCGCGCCG 6001 AGGTGAAGTT CGAGGGCGAC ACCCTGGTGA ACCGCATCGA GCTGAAGGGC ATCGACTTCA 6061 AGGAGGACGG CAACATCCTG GGGCACAAGC TGGAGTACAA CTACAACAGC CACAACGTCT 6121 ATATCACCGC CGACAAGCAG AAGAACGGCA TCAAGGCCAA CTTCAAGATC CGCCACAACA 6181 TCGAGGACGG CAGCGTGCAG CTCGCCGACC ACTACCAGCA GAACACCCCC ATCGGCGACG 6241 GCCCCGTGCT GCTGCCCGAC AACCACTACC TGAGCCACCA GTCCGCCCTG AGCAAAGACC 6301 CCAACGAGAA GCGCGATCAC ATGGTCCTGC TGGAGTTCGT GACCGCCGCC GGGATCACTC 6361 TCGGCATGGA CGAGCTGTAC AAGTAAggcg cgccgtacgc gatcgtaagc ttgatgagtt 6421 tggacaaacc acaactagaa tgcagtgaaa aaaatgcttt atttgtgaaa tttgtgatgc 6481 tattgcttta tttgtaacca ttataagctg caataaacaa gttaacaaca acaattgcat 6541 tcattttatg tttcaggttc agggggaagt gtgggaggtt ttttaaagca agtaaaacct 6601 ctacaaatgt ggtatggctg attatgatct agagtcgcgg ccgccttaac gaaataatta 6661 attgtttcca ttcgacacga tattggtgct atttgccgac ccccaattgc acaaatgact 6721 tctcatagac gtagacaagg gggggatgta ggaccgtggt atcaaaaacg tcgataaatt 6781 gaaaaggtcc acgaaaactt tataccgatc gctgaaaaac aaaatcaaac attgcaaaaa 6841 ccgtgttgaa atcaatttca ccttaacgtt gaccttaaat actgataccc cgaagagccc 6901 cctgttccta actgcgaaga accaatcatt ctttgcacca tcaaaacatg attatctgaa 6961 attataaaat ttaaacaaaa acaattgacg tacggattta ctaacaccgc catttcgtga 7021 tcagcgagtc tatgtccatc aaaagtgtta gaatttggtg gggctgactg aacctgggtt 7081 cgtccctgta aaaggtgatc atgatagccc cttgcagggc tttgtgtgag aagcgcctct 7141 cgccgcgcga cattagagcc tcatggacct caactttaaa aatcgactcg taaacctcgc 7201 gtctcttctc caaatcgttc aatttgtaac gctttaaagt ctccgcctcc tccttctccg 7261 ccagctcttt ttgctcggtt aaaagcttct cgaccgcttt ttggtacttc ccccagaaat 7321 taaacccctc gagctccagc cccggcgttc gctccagcca ccgctggacc aaatcggtca 7381 aagacggctc tttctccgac ttggcgattt gctcgagcgc tttttcgtca tttccgaaca 7441 ctttcgagta gttctggttg tatttgacgc ggttttcctg gcgcaccccg agcttgtttt 7501 ccaggagcct gaactgcagg ctctggaacc cggaagcggg ccgcagataa caccggaagt 7561 ccatgaaatc gagcggtgtc atcgtttcca gaatcatcac ttggtccacc aagacctaca 7621 attgcgacag ttttatctcg tgaccgcaat ttgttgcgaa attgtgaacc ttgaggatca 7681 aaacaacacg attgaggcgt ttgaggattt ccaaagtttg cgactcttct aaaacgtcgc 7741 tgaaaatgtt gcggatcgag tccagctcgt agattatttg tttgaaccat aactcatacg 7801 ctggaattac ttgttatagt taattacggt taagtgggtt ttttaccttg atgggtcaca 7861 ataaataaat gctcgtcgtg cacgggctgg ttattttgct cgctgagtaa tctttgggct 7921 tctaaaatct tgtccaacat caagtattca ccgtaaagca tcccacattc ttcgctcagt 7981 tggtcgcctt cttgggcttc acttaattta cgattaattg taaatggaat aattaaatta 8041 aatcatactt acgagggtct cagtgggcaa ctcattacga ccgcggtacc gtcgacgttc 8101 tagcggtacc ccgattgttt agcttgttca gctgcgcttg tttatttgct tagctttcgc 8161 ttagcgacgt gttcactttg cttgtttgaa ttgaattgtc gctccgtaga cgaagcgcct 8221 ctatttatac tccggcggtc gagggttcga aatcgataag cttggatcct aattgaatta 8281 gctctaattg aattagtctc taattgaatt agatccccgg gcgagctcga attaattcaa $8341 \mathrm{c}$

// 


\section{P3 8695...8685 \\ P3 8679...8669 \\ P3 8664...8654 \\ 3XP3ITATA 8695...8607 \\ TATA $8628 \ldots 8607$}

pBacR 21...1050

Tc'vermilionIgenomic 8468...7030

SV40polyA 7019...6797

H2Av::C3PA-GFP 5667...6770

alphaTub1 promoter $5213 \ldots 5648$

pBacL 5206...4489

Figure S7.23 pB[3xP3-gTc'v;Tc' $\alpha$ Tub1P-Tc'H2Av:: C3PA-GFP].

Sequence S7.24 pB[3xP3-gTc'v;Tc' $\alpha$ Tub1P- Tc'H2Av:: C3PA-GFP], GenBank formatted.

LOCUS pBac_3xP3_gTcv_alp 8725 bp ds-DNA circular 05-MAY-2016

SOURCE

ORGANISM

COMMENT

COMMENT ApEinfo:methylated:1

FEATURES Location/Qualifiers

promoter complement(8607..8695)

/vntifkey="29"

/label=3xP3\TATA

/ApEinfo_fwdcolor=\#ffffff

/ApEinfo_revcolor=SystemWindow

misc_feature complement(6797..7019)

/vntifkey="21"

/label=SV40polyA

/ApEinfo_fwdcolor=\#ff8080

/ApEinfo_revcolor=\#ffOOff

misc structure 21..1050

/vntifkey="88"

/label=pBacR

/ApEinfo_fwdcolor=pink

/ApEinfo_revcolor=pink

misc_structure complement(4489..5206)

/vntifkey="88"

/label=pBacL

/ApEinfo_fwdcolor=pink

/ApEinfo_revcolor=pink

misc_signal complement(7030..8468)

/vntifkey="87"

/label=Tc'vermilion\genomic

/ApEinfo_fwdcolor=pink

/ApEinfo_revcolor=pink

misc_feature complement(8685..8695)

/vntifkey="21"

/label=P3

/ApEinfo_fwdcolor=\#ff8080

/ApEinfo_revcolor=\#ffOOff 
misc_feature complement(8669..8679)

/vntifkey="21"

/label=P3(1)

/ApEinfo_label=P3

/ApEinfo_fwdcolor=\#ff8080

/ApEinfo_revcolor=\#ffOOff

misc_feature complement(8654..8664)

/vntifkey="21"

/label=P3(2)

/ApEinfo_label=P3

/ApEinfo_fwdcolor=\#ff8080

/ApEinfo_revcolor=\#ffOOff

misc_feature complement(8607..8628)

/vntifkey="21"

/label=TATA

/ApEinfo_fwdcolor=\#ff8080

/ApEinfo_revcolor=\#ffOOff

misc_feature 5213..5648

/vntifkey="21"

/label=alphaTub1 promoter

/ApEinfo_fwdcolor=\#ff8080

/ApEinfo_revcolor=\#ffooff

misc_feature 2331..3191

/label=Amp (R)

/ApEinfo_fwdcolor=\#ff8000

/ApEinfo_revcolor=green

misc feature $5667 . .6770$

/label=H2Av::C3PA-GFP

/ApEinfo_fwdcolor=\#80ff80

/ApEinfo_revcolor=green

ORIGIN

1 cattgtggga accgtgcgat caaacaaacg cgagataccg gaagtactga aaaacagtcg

61 ctccaggcca gtgggaacat cgatgttttg ttttgacgga ccccttactc tcgtctcata

121 taaaccgaag ccagctaaga tggtatactt attatcatct tgtgatgagg atgcttctat

181 caacgaaagt accggtaaac cgcaaatggt tatgtattat aatcaaacta aaggcggagt

241 ggacacgcta gaccaaatgt gttctgtgat gacctgcagt aggaagacga ataggtggcc

301 tatggcatta ttgtacggaa tgataaacat tgcctgcata aattctttta ttatatacag

361 ccataatgtc agtagcaagg gagaaaaggt tcaaagtcgc aaaaaattta tgagaaacct

421 ttacatgagc ctgacgtcat cgtttatgcg taagcgttta gaagctccta ctttgaagag

481 atatttgcgc gataatatct ctaatatttt gccaaatgaa gtgcctggta catcagatga

541 cagtactgaa gagccagtaa tgaaaaaacg tacttactgt acttactgcc cctctaaaat

601 aaggcgaaag gcaaatgcat cgtgcaaaaa atgcaaaaaa gttatttgtc gagagcataa

661 tattgatatg tgccaaagtt gtttctgact gactaataag tataatttgt ttctattatg

721 tataagttaa gctaattact tattttataa tacaacatga ctgtttttaa agtacaaaat

781 aagtttattt ttgtaaaaga gagaatgttt aaaagttttg ttactttata gaagaaattt

841 tgagtttttg ttttttttta ataaataaat aaacataaat aaattgtttg ttgaatttat

901 tattagtatg taagtgtaaa tataataaaa cttaatatct attcaaatta ataaataaac

961 ctcgatatac agaccgataa aacacatgcg tcaattttac gcatgattat ctttaacgta

1021 cgtcacaata tgattatctt tctagggtta aataatagtt tctaattttt ttattattca

1081 gcctgctgtc gtgaataccg tatatctcaa cgctgtctgt gagattgtcg tattctagcc

$1141 \mathrm{tttttagttt} \mathrm{ttcgctcatc} \mathrm{gacttgatat} \mathrm{tgtccgacac} \mathrm{attttcgtcg} \mathrm{atttgcgttt}$

1201 tgatcaaaga cttgagcaga gacacgttaa tcaactgttc aaattgatcc atattaacga

1261 tatcaacccg atgcgtatat ggtgcgtaaa atatattttt taaccctctt atactttgca

1321 ctctgcgtta atacgcgttc gtgtacagac gtaatcatgt tttctttttt ggataaaact

1381 cctactgagt ttgacctcat attagaccct cacaagttgc aaaacgtggc attttttacc

1441 aatgaagaat ttaaagttat tttaaaaaat ttcatcacag atttaaagaa gaaccaaaaa

1501 ttaaattatt taatcgacca gttaatcaac gtgtacacag agcgcaaaaa acacgcagcc

1561 cgacgtgttg gctaaaatta ttaaatcaac ttgtgttata gtcacgattt gccgtccaac

1621 gtgttcctca aaaagttgaa gaccaacaag tttacggaca ctagttaatt atttgatttt

1681 gccccacttc attttgtggg atcacaattt tgttatattt taaacaaagc ttggcactgg

1741 ccgtcgtttt acaacgtcgt gactgggaaa accctggcgt tacccaactt aatcgccttg

1801 cagcacatcc ccctttcgcc agctggcgta atagcgaaga ggcccgcacc gatcgccctt

1861 cccaacagtt gcgcagcctg aatggcgaat ggcgcctgat gcggtatttt ctccttacgc 
1921 atctgtgcgg tatttcacac cgcatatggt gcactctcag tacaatctgc tctgatgccg 1981 catagttaag ccagccccga cacccgccaa cacccgctga cgcgccctga cgggcttgtc 2041 tgctcccggc atccgcttac agacaagctg tgaccgtctc cgggagctgc atgtgtcaga 2101 ggttttcacc gtcatcaccg aaacgcgcga gacgaaaggg cctcgtgata cgcctatttt 2161 tataggttaa tgtcatgata ataatggttt cttagacgtc aggtggcact tttcggggaa 2221 atgtgcgcgg aacccctatt tgtttatttt tctaaataca ttcaaatatg tatccgctca 2281 tgagacaata accctgataa atgcttcaat aatattgaaa aaggaagagt atgagtattc 2341 aacatttccg tgtcgccctt attccctttt ttgcggcatt ttgccttcct gtttttgctc 2401 acccagaaac gctggtgaaa gtaaaagatg ctgaagatca gttgggtgca cgagtgggtt 2461 acatcgaact ggatctcaac agcggtaaga tccttgagag ttttcgcccc gaagaacgtt 2521 ttccaatgat gagcactttt aaagttctgc tatgtggcgc ggtattatcc cgtattgacg 2581 ccgggcaaga gcaactcggt cgccgcatac actattctca gaatgacttg gttgagtact 2641 caccagtcac agaaaagcat cttacggatg gcatgacagt aagagaatta tgcagtgctg 2701 ccataaccat gagtgataac actgcggcca acttacttct gacaacgatc ggaggaccga 2761 aggagctaac cgcttttttg cacaacatgg gggatcatgt aactcgcctt gatcgttggg 2821 aaccggagct gaatgaagcc ataccaaacg acgagcgtga caccacgatg cctgtagcaa 2881 tggcaacaac gttgcgcaaa ctattaactg gcgaactact tactctagct tcccggcaac 2941 aattaataga ctggatggag gcggataaag ttgcaggacc acttctgcgc tcggcccttc 3001 cggctggctg gtttattgct gataaatctg gagccggtga gcgtgggtct cgcggtatca 3061 ttgcagcact ggggccagat ggtaagccct cccgtatcgt agttatctac acgacgggga 3121 gtcaggcaac tatggatgaa cgaaatagac agatcgctga gataggtgcc tcactgatta 3181 agcattggta actgtcagac caagtttact catatatact ttagattgat ttaaaacttc 3241 atttttaatt taaaaggatc taggtgaaga tcctttttga taatctcatg accaaaatcc 3301 cttaacgtga gttttcgttc cactgagcgt cagaccccgt agaaaagatc aaaggatctt 3361 cttgagatcc tttttttctg cgcgtaatct gctgcttgca aacaaaaaaa ccaccgctac 3421 cagcggtggt ttgtttgccg gatcaagagc taccaactct ttttccgaag gtaactggct 3481 tcagcagagc gcagatacca aatactgtcc ttctagtgta gccgtagtta ggccaccact 3541 tcaagaactc tgtagcaccg cctacatacc tcgctctgct aatcctgtta ccagtggctg 3601 ctgccagtgg cgataagtcg tgtcttaccg ggttggactc aagacgatag ttaccggata 3661 aggcgcagcg gtcgggctga acggggggtt cgtgcacaca gcccagcttg gagcgaacga 3721 cctacaccga actgagatac ctacagcgtg agcattgaga aagcgccacg cttcccgaag 3781 ggagaaaggc ggacaggtat ccggtaagcg gcagggtcgg aacaggagag cgcacgaggg 3841 agcttccagg gggaaacgcc tggtatcttt atagtcctgt cgggtttcgc cacctctgac 3901 ttgagcgtcg atttttgtga tgctcgtcag gggggeggag cctatggaaa aacgccagca 3961 acgcggcctt tttacggttc ctggcctttt gctggccttt tgctcacatg ttctttcctg 4021 cgttatcccc tgattctgtg gataaccgta ttaccgcctt tgagtgagct gataccgctc 4081 gccgcagccg aacgaccgag cgcagcgagt cagtgagcga ggaagcggaa gagcgcccaa 4141 tacgcaaacc gcctctcccc gcgcgttggc cgattcatta atgcagctgg cacgacaggt 4201 ttcccgactg gaaagcgggc agtgagcgca acgcaattaa tgtgagttag ctcactcatt 4261 aggcacccca ggctttacac tttatgcttc cggctcgtat gttgtgtgga attgtgagcg 4321 gataacaatt tcacacagga aacagctatg accatgatta cgaattcgag ctcggtaccc 4381 ggggatcctc tagagtcgac gctcgcgcga cttggtttgc cattctttag cgcgcgtcgc 4441 gtcacacagc ttggccacaa tgtggttttt gtcaaacgaa gattctatga cgtgtttaaa 4501 gtttaggtcg agtaaagcgc aaatcttttt taaccctaga aagatagtct gcgtaaaatt 4561 gacgcatgca ttcttgaaat attgctctct ctttctaaat agcgcgaatc cgtcgctgtg 4621 catttaggac atctcagtcg ccgcttggag ctcccgtgag gcgtgcttgt caatgcggta 4681 agtgtcactg attttgaact ataacgaccg cgtgagtcaa aatgacgcat gattatcttt 4741 tacgtgactt ttaagattta actcatacga taattatatt gttatttcat gttctactta 4801 cgtgataact tattatatat atattttctt gttatagata tcgtgactaa tatataataa 4861 aatgggtagt tctttagacg atgagcatat cctctctgct cttctgcaaa gcgatgacga 4921 gcttgttggt gaggattctg acagtgaaat atcagatcac gtaagtgaag atgacgtcca 4981 gagcgataca gaagaagcgt ttatagatga ggtacatgaa gtgcagccaa cgtcaagcgg 5041 tagtgaaata ttagacgaac aaaatgttat tgaacaacca ggttcttcat tggcttctaa 5101 cagaatcttg accttgccac agaggactat tagaggtaag aataaacatt gttggtcaac 5161 ttcaaagtcc acgaggcgta gccgagtctc tgcactgaac attgtcagat ctcactgcag 5221 tgaacggtta tgatggtgca acgaccggga cctcagctgt ataaaaaatc tgaaaataat 5281 tgataatggt cgaagaccat aaggaaaaat aattcgaaaa ttaaaggcaa agtatgaaaa 5341 tgctggaaat aaatcgggaa acaccgaatt tttggtcaca ggaaccaacg aatttttttt 5401 tgaatattga tttgaaaaga gatcgagaac aaggataaca atcttccgtt agagaccgtt 5461 ggtaccgaca tggcggggag ccaatcagaa gtctggaaat cgggcgttgg ttgctgacgt 5521 caacaagctt ataaatagca acgacagttg aaaatcgaat caaagtcgtt tggaaaaagc 5581 cagagcttgt atttccgaag cgtactcccg tttttctgct cttttgtggt gtaatttgta 5641 aaactcaagg ccggccCTAA AGAAAAATGG CTGGTGGCAA AGCAGGGAAA GATTCGGGCA 
5701 AAGCTAAAGC CAAGGCGGTG TCGCGCTCGG CGCGTGCCGG ACTCCAGTTC CCCGTGGGGA 5761 GGATCCACCG CCACTTGAAG AATCGCACTA CGAGCCATGG ACGCGTGGGG GCCACCGCGG 5821 CTGTGTATTC CGCCGCGATT TTGGAGTATT TGACAGCTGA GGTCCTGGAG TTGGCCGGGA 5881 ACGCCAGTAA GGACTTGAAG GTGAAGAGGA TCACCCCCAG GCACTTGCAG CTGGCCATCA 5941 GAGGGGATGA GGAGCTGGAC AGCCTGATCA AGGCCACTAT CGCCGGCGGA GGCGTCATTC 6001 CACACATCCA CAAGAGTTTG ATCGGGAAAA AGGGCCACTC ACAGCCCGTC ATGGTGAGCA 6061 AGGGCGAGGA GCTGTTCACC GGGGTGGTGC CCATCCTGGT CGAGCTGGAC GGCGACGTAA 6121 ACGGCCACAA GTTCAGCGTG TCCGGCGAGG GCGAGGGCGA TGCCACCTAC GGCAAGCTGA 6181 CCCTGAAGTT CATCTGCACC ACCGGCAAGC TGCCCGTGCC CTGGCCCACC CTCGTGACCA 6241 CCTTCAGCTA CGGCGTGCAG TGCTTCAGCC GCTACCCCGA CCACATGAAG CAGCACGACT 6301 TCTTCAAGTC CGCCATGCCC GAAGGCTACG TCCAGGAGCG CACCATCTCC TTCAAGGACG 6361 ACGGCAACTA CAAGACCCGC GCCGAGGTGA AGTTCGAGGG CGACACCCTG GTGAACCGCA 6421 TCGAGCTGAA GGGCATCGAC TTCAAGGAGG ACGGCAACAT CCTGGGGCAC AAGCTGGAGT 6481 ACAACTACAA CAGCCACAAC GTCTATATCA CCGCCGACAA GCAGAAGAAC GGCATCAAGG 6541 CCAACTTCAA GATCCGCCAC AACATCGAGG ACGGCAGCGT GCAGCTCGCC GACCACTACC 6601 AGCAGAACAC CCCCATCGGC GACGGCCCCG TGCTGCTGCC CGACAACCAC TACCTGAGCC 6661 ACCAGTCCGC CCTGAGCAAA GACCCCAACG AGAAGCGCGA TCACATGGTC CTGCTGGAGT 6721 TCGTGACCGC CGCCGGGATC ACTCTCGGCA TGGACGAGCT GTACAAGTAA ggcgcgccgt

6781 acgcgatcgt aagcttgatg agtttggaca aaccacaact agaatgcagt gaaaaaaatg 6841 ctttatttgt gaaatttgtg atgctattgc tttatttgta accattataa gctgcaataa 6901 acaagttaac aacaacaatt gcattcattt tatgtttcag gttcaggggg aagtgtggga 6961 ggttttttaa agcaagtaaa acctctacaa atgtggtatg gctgattatg atctagagtc 7021 gcggccgcct taacgaaata attaattgtt tccattcgac acgatattgg tgctatttgc 7081 cgacccccaa ttgcacaaat gacttctcat agacgtagac aaggggggga tgtaggaccg 7141 tggtatcaaa aacgtcgata aattgaaaag gtccacgaaa actttatacc gatcgctgaa 7201 aaacaaaatc aaacattgca aaaaccgtgt tgaaatcaat ttcaccttaa cgttgacctt 7261 aaatactgat accccgaaga gccccctgtt cctaactgcg aagaaccaat cattctttgc 7321 accatcaaaa catgattatc tgaaattata aaatttaaac aaaaacaatt gacgtacgga 7381 tttactaaca ccgccatttc gtgatcagcg agtctatgtc catcaaaagt gttagaattt 7441 ggtggggctg actgaacctg ggttcgtccc tgtaaaaggt gatcatgata gccccttgca 7501 gggctttgtg tgagaagcgc ctctcgccgc gcgacattag agcctcatgg acctcaactt 7561 taaaaatcga ctcgtaaacc tcgcgtctct tctccaaatc gttcaatttg taacgcttta 7621 aagtctccgc ctcctccttc tccgccagct ctttttgctc ggttaaaagc ttctcgaccg 7681 ctttttggta cttcccccag aaattaaacc cctcgagctc cagccccggc gttcgctcca 7741 gccaccgctg gaccaaatcg gtcaaagacg gctctttctc cgacttggcg atttgctcga 7801 gcgctttttc gtcatttccg aacactttcg agtagttctg gttgtatttg acgcggtttt 7861 cctggcgcac cccgagcttg ttttccagga gcctgaactg caggctctgg aacccggaag 7921 cgggccgcag ataacaccgg aagtccatga aatcgagcgg tgtcatcgtt tccagaatca 7981 tcacttggtc caccaagacc tacaattgcg acagttttat ctcgtgaccg caatttgttg 8041 cgaaattgtg aaccttgagg atcaaaacaa cacgattgag gcgtttgagg atttccaaag 8101 tttgcgactc ttctaaaacg tcgctgaaaa tgttgcggat cgagtccagc tcgtagatta 8161 tttgtttgaa ccataactca tacgctggaa ttacttgtta tagttaatta cggttaagtg 8221 ggttttttac cttgatgggt cacaataaat aaatgctcgt cgtgcacggg ctggttattt 8281 tgctcgctga gtaatctttg ggcttctaaa atcttgtcca acatcaagta ttcaccgtaa 8341 agcatcccac attcttcgct cagttggtcg ccttcttggg cttcacttaa tttacgatta 8401 attgtaaatg gaataattaa attaaatcat acttacgagg gtctcagtgg gcaactcatt 8461 acgaccgcgg taccgtcgac gttctagcgg taccccgatt gtttagcttg ttcagctgcg 8521 cttgtttatt tgcttagctt tcgcttagcg acgtgttcac tttgcttgtt tgaattgaat 8581 tgtcgctccg tagacgaagc gcctctattt atactccggc ggtcgagggt tcgaaatcga 8641 taagcttgga tcctaattga attagctcta attgaattag tctctaattg aattagatcc // 8701 ccgggcgagc tcgaattaat tcaac 


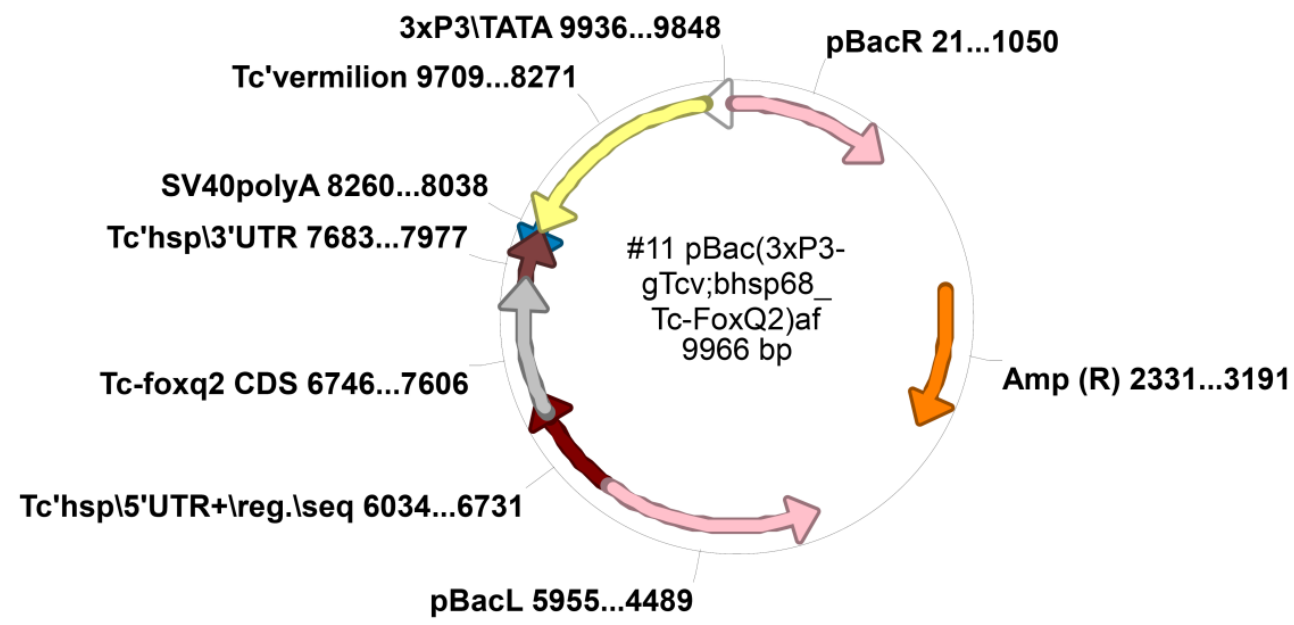

Figure S7.25 pB[3xP3-gTc'v;Tc'hsp68-Tc'foxq2].

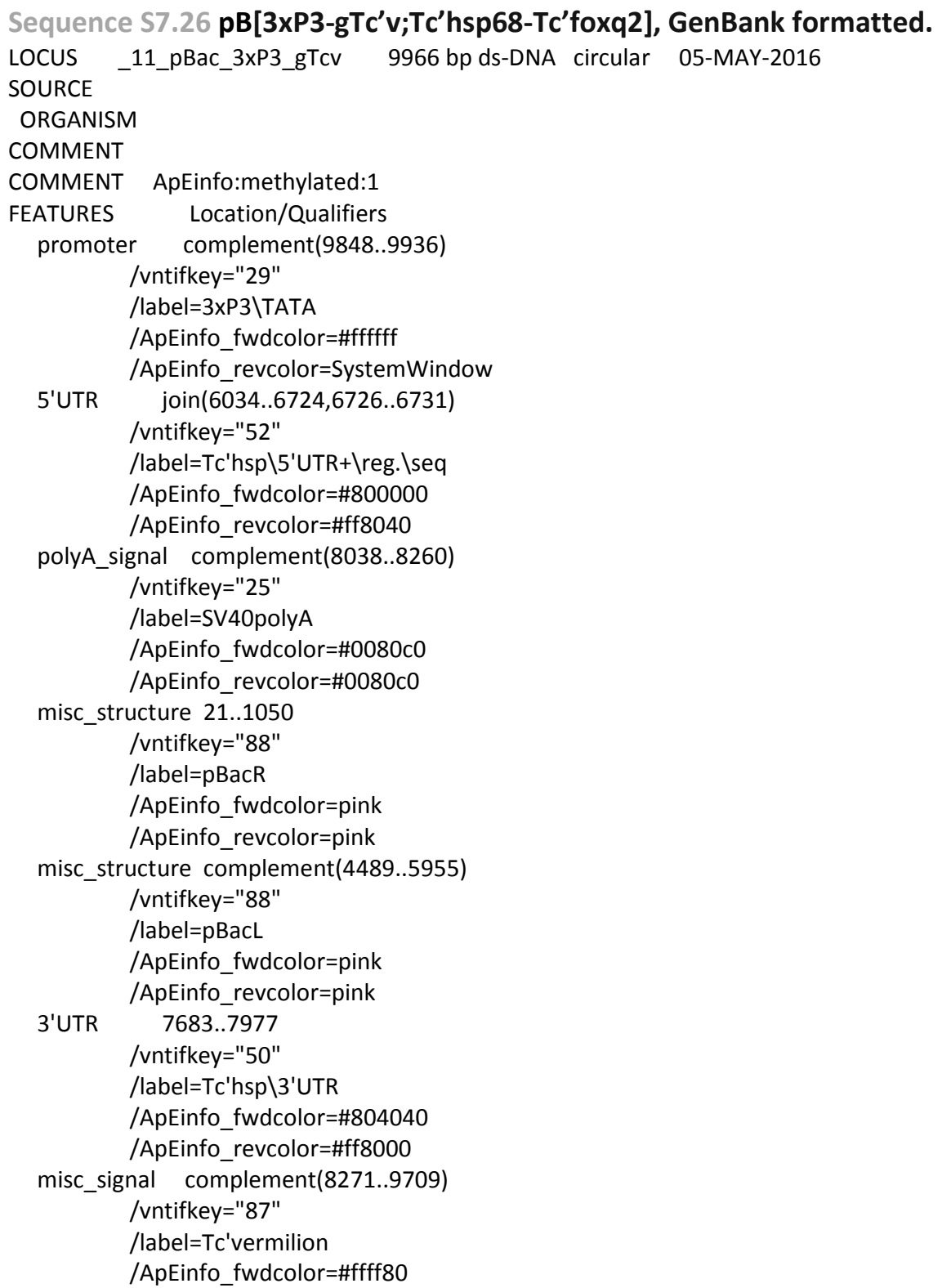


/ApEinfo_revcolor=\#ffff80

misc_feature 2331..3191

/label=Amp (R)

/ApEinfo_fwdcolor=\#ff8000

/ApEinfo_revcolor=green

misc_feature $67 \overline{4} 6 . .7606$

/label=Tc-foxq2 CDS

/ApEinfo_fwdcolor=\#c0c0c0

ORIGIN

/ApEinfo_revcolor=green

1 cattgtggga accgtgcgat caaacaaacg cgagataccg gaagtactga aaaacagtcg

61 ctccaggcca gtgggaacat cgatgttttg ttttgacgga ccccttactc tcgtctcata

121 taaaccgaag ccagctaaga tggtatactt attatcatct tgtgatgagg atgcttctat

181 caacgaaagt accggtaaac cgcaaatggt tatgtattat aatcaaacta aaggcggagt

241 ggacacgcta gaccaaatgt gttctgtgat gacctgcagt aggaagacga ataggtggcc

301 tatggcatta ttgtacggaa tgataaacat tgcctgcata aattctttta ttatatacag

361 ccataatgtc agtagcaagg gagaaaaggt tcaaagtcgc aaaaaattta tgagaaacct

421 ttacatgagc ctgacgtcat cgtttatgcg taagcgttta gaagctccta ctttgaagag

481 atatttgcgc gataatatct ctaatatttt gccaaatgaa gtgcctggta catcagatga

541 cagtactgaa gagccagtaa tgaaaaaacg tacttactgt acttactgcc cctctaaaat

601 aaggcgaaag gcaaatgcat cgtgcaaaaa atgcaaaaaa gttatttgtc gagagcataa

661 tattgatatg tgccaaagtt gtttctgact gactaataag tataatttgt ttctattatg

721 tataagttaa gctaattact tattttataa tacaacatga ctgtttttaa agtacaaaat

781 aagtttattt ttgtaaaaga gagaatgttt aaaagttttg ttactttata gaagaaattt

841 tgagtttttg ttttttttta ataaataaat aaacataaat aaattgtttg ttgaatttat

901 tattagtatg taagtgtaaa tataataaaa cttaatatct attcaaatta ataaataaac

961 ctcgatatac agaccgataa aacacatgcg tcaattttac gcatgattat ctttaacgta

1021 cgtcacaata tgattatctt tctagggtta aataatagtt tctaattttt ttattattca

1081 gcctgctgtc gtgaataccg tatatctcaa cgctgtctgt gagattgtcg tattctagcc

$1141 \mathrm{tttttagttt} \mathrm{ttcgctcatc} \mathrm{gacttgatat} \mathrm{tgtccgacac} \mathrm{attttcgtcg} \mathrm{atttgcgttt}$

1201 tgatcaaaga cttgagcaga gacacgttaa tcaactgttc aaattgatcc atattaacga

1261 tatcaacccg atgcgtatat ggtgcgtaaa atatattttt taaccctctt atactttgca

1321 ctctgcgtta atacgcgttc gtgtacagac gtaatcatgt tttctttttt ggataaaact

1381 cctactgagt ttgacctcat attagaccct cacaagttgc aaaacgtggc attttttacc

1441 aatgaagaat ttaaagttat tttaaaaaat ttcatcacag atttaaagaa gaaccaaaaa

1501 ttaaattatt taatcgacca gttaatcaac gtgtacacag agcgcaaaaa acacgcagcc

1561 cgacgtgttg gctaaaatta ttaaatcaac ttgtgttata gtcacgattt gccgtccaac

1621 gtgttcctca aaaagttgaa gaccaacaag tttacggaca ctagttaatt atttgatttt

1681 gccccacttc attttgtggg atcacaattt tgttatattt taaacaaagc ttggcactgg

1741 ccgtcgtttt acaacgtcgt gactgggaaa accctggcgt tacccaactt aatcgccttg

1801 cagcacatcc ccctttcgcc agctggcgta atagcgaaga ggcccgcacc gatcgccctt

1861 cccaacagtt gcgcagcctg aatggcgaat ggcgcctgat gcggtatttt ctccttacgc

1921 atctgtgcgg tatttcacac cgcatatggt gcactctcag tacaatctgc tctgatgccg

1981 catagttaag ccagccccga cacccgccaa cacccgctga cgcgccctga cgggcttgtc

2041 tgctcccggc atccgcttac agacaagctg tgaccgtctc cgggagctgc atgtgtcaga

2101 ggttttcacc gtcatcaccg aaacgcgcga gacgaaaggg cctcgtgata cgcctatttt

2161 tataggttaa tgtcatgata ataatggttt cttagacgtc aggtggcact tttcggggaa

2221 atgtgcgcgg aacccctatt tgtttatttt tctaaataca ttcaaatatg tatccgctca

2281 tgagacaata accctgataa atgcttcaat aatattgaaa aaggaagagt atgagtattc

2341 aacatttccg tgtcgccctt attccctttt ttgcggcatt ttgccttcct gtttttgctc

2401 acccagaaac gctggtgaaa gtaaaagatg ctgaagatca gttgggtgca cgagtgggtt

2461 acatcgaact ggatctcaac agcggtaaga tccttgagag ttttcgcccc gaagaacgtt

2521 ttccaatgat gagcactttt aaagttctgc tatgtggcgc ggtattatcc cgtattgacg

2581 ccgggcaaga gcaactcggt cgccgcatac actattctca gaatgacttg gttgagtact

2641 caccagtcac agaaaagcat cttacggatg gcatgacagt aagagaatta tgcagtgctg

2701 ccataaccat gagtgataac actgcggcca acttacttct gacaacgatc ggaggaccga

2761 aggagctaac cgcttttttg cacaacatgg gggatcatgt aactcgcctt gatcgttggg

2821 aaccggagct gaatgaagcc ataccaaacg acgagcgtga caccacgatg cctgtagcaa 2881 tggcaacaac gttgcgcaaa ctattaactg gcgaactact tactctagct tcccggcaac 2941 aattaataga ctggatggag gcggataaag ttgcaggacc acttctgcgc tcggcccttc 3001 cggctggctg gtttattgct gataaatctg gagccggtga gcgtgggtct cgcggtatca 3061 ttgcagcact ggggccagat ggtaagccct cccgtatcgt agttatctac acgacgggga 3121 gtcaggcaac tatggatgaa cgaaatagac agatcgctga gataggtgcc tcactgatta 
3181 agcattggta actgtcagac caagtttact catatatact ttagattgat ttaaaacttc 3241 atttttaatt taaaaggatc taggtgaaga tcctttttga taatctcatg accaaaatcc 3301 cttaacgtga gttttcgttc cactgagcgt cagaccccgt agaaaagatc aaaggatctt 3361 cttgagatcc tttttttctg cgcgtaatct gctgcttgca aacaaaaaa ccaccgctac 3421 cagcggtggt ttgtttgccg gatcaagagc taccaactct ttttccgaag gtaactggct 3481 tcagcagagc gcagatacca aatactgtcc ttctagtgta gccgtagtta ggccaccact 3541 tcaagaactc tgtagcaccg cctacatacc tcgctctgct aatcctgtta ccagtggctg 3601 ctgccagtgg cgataagtcg tgtcttaccg ggttggactc aagacgatag ttaccggata 3661 aggcgcagcg gtcgggctga acggggggtt cgtgcacaca gcccagcttg gagcgaacga 3721 cctacaccga actgagatac ctacagcgtg agcattgaga aagcgccacg cttcccgaag 3781 ggagaaaggc ggacaggtat ccggtaagcg gcagggtcgg aacaggagag cgcacgaggg 3841 agcttccagg gggaaacgcc tggtatcttt atagtcctgt cgggtttcgc cacctctgac 3901 ttgagcgtcg atttttgtga tgctcgtcag gggggcggag cctatggaaa aacgccagca 3961 acgcggcctt tttacggttc ctggcctttt gctggccttt tgctcacatg ttctttcctg 4021 cgttatcccc tgattctgtg gataaccgta ttaccgcctt tgagtgagct gataccgctc 4081 gccgcagccg aacgaccgag cgcagcgagt cagtgagcga ggaagcggaa gagcgcccaa 4141 tacgcaaacc gcctctcccc gcgcgttggc cgattcatta atgcagctgg cacgacaggt 4201 ttcccgactg gaaagcggge agtgagcgca acgcaattaa tgtgagttag ctcactcatt 4261 aggcacccca ggctttacac tttatgcttc cggctcgtat gttgtgtgga attgtgagcg 4321 gataacaatt tcacacagga aacagctatg accatgatta cgaattcgag ctcggtaccc 4381 ggggatcctc tagagtcgac gctcgcgcga cttggtttgc cattctttag cgcgcgtcgc 4441 gtcacacagc ttggccacaa tgtggttttt gtcaaacgaa gattctatga cgtgtttaaa 4501 gtttaggtcg agtaaagcgc aaatcttttt taaccctaga aagatagtct gcgtaaaatt 4561 gacgcatgca ttcttgaaat attgctctct ctttctaaat agcgcgaatc cgtcgctgtg 4621 catttaggac atctcagtcg ccgcttggag ctcccgtgag gcgtgcttgt caatgcggta 4681 agtgtcactg attttgaact ataacgaccg cgtgagtcaa aatgacgcat gattatcttt 4741 tacgtgactt ttaagattta actcatacga taattatatt gttatttcat gttctactta 4801 cgtgataact tattatatat atattttctt gttatagata tcgtgactaa tatataataa 4861 aatgggtagt tctttagacg atgagcatat cctctctgct cttctgcaaa gcgatgacga 4921 gcttgttggt gaggattctg acagtgaaat atcagatcac gtaagtgaag atgacgtcca 4981 gagcgataca gaagaagcgt ttatagatga ggtacatgaa gtgcagccaa cgtcaagcgg 5041 tagtgaaata ttagacgaac aaaatgttat tgaacaacca ggttcttcat tggcttctaa 5101 cagaatcttg accttgccac agaggactat tagaggtaag aataaacatt gttggtcaac 5161 ttcaaagtcc acgaggcgta gccgagtctc tgcactgaac attgtcagat ctcaaagagg 5221 tccgacgcgt atgtgccgca atatatatga cccactttta tgcttcaaac tattttttac 5281 tgatgagata atttcggaaa ttgtaaaatg gacaaatgct gagatatcat tgaaacgtcg 5341 ggaatctatg acaggtgcta catttcgtga cacgaatgaa gatgaaatct atgctttctt 5401 tggtattctg gtaatgacag cagtgagaaa agataaccac atgtccacag atgacctctt 5461 tgatcgatct ttgtcaatgg tgtacgtctc tgtaatgagt cgtgatcgtt ttgatttttt 5521 gatacgatgt cttagaatgg atgacaaaag tatacggccc acacttcgag aaaacgatgt 5581 atttactcct gttagaaaaa tatgggatct ctttatccat cagtgcatac aaaattacac 5641 tccaggggct catttgacca tagatgaaca gttacttggt tttagaggac ggtgtccgtt 5701 taggatgtat atcccaaaca agccaagtaa gtatggaata aaaatcctca tgatgtgtga 5761 cagtggtacg aagtatatga taaatggaat gccttatttg ggaagaggaa cacagaccaa 5821 cggagtacca ctcggtgaat actacgtgaa ggagttatca aagcctgtgc acggtagttg 5881 tcgtaatatt acgtgtgaca attggttcac ctcaatccct ttggcaaaaa acttactaca 5941 agaaccgtat aagttcgaga tcggccggcc gaattcgaat ggccatggga cgtcgacctg 6001 aggtaattat aacccgggcc ctatatatgg atcccaacct tcaataaaaa attgttcttt 6061 atttattatt aatcactatt atttaggcat cttctgcaaa agtaattgat tataaacgat 6121 aaaagtaaaa aaaaggatta atctaaaaca ttttacttaa agacctatta ataagtttaa 6181 aaagaaaaaa aataggaaaa ttgttttatt aaaattgcta tgacaatcca actttggcat 6241 atgtatatga aatcaaaaca attcactact cattcataat attgccgaac tttaccaaat 6301 taaaaataaa catgaaatag tcaacaactc attcgttatg taacgttttt tttttaaatg 6361 cattaatata taatgattgt ttttattttg gaagttgaaa taaattaggg cacaaccagt 6421 ttccactatt ctcctttatc tttacggaaa tagcatgaat aataccgaaa aggccgtttt 6481 aaaaataaaa attcgagaaa attcaaacgc aaatcaatgg aaacttcgag atcattcggt 6541 gaagttcgag acgaattccc cgtttcatat ataagcgcgg tctcgcggcg cgttgtcagt 6601 gaatttgaat caagcgaagc gaaagcaaca aagctaagca agcgactcaa agcaaatttt 6661 caaagcgact ttaacaaagt tgagtgaatt atttactagt gaattcaaag taaatcgaaa 6721 agtgtatcta gaggtaccgg ttgttATGTG CAGTAACGAG ACTCCGGCCA ACACCTCACC 6781 GAGGCTGCCG ATGCCCTTCG CGCTCGAAGG TGGCCCCCGG GCCCTGCTGC CCATCGACCA 6841 GTACCGGCTC CAGCTCTACC AGTATGCCGT CGCAGAAAGG CTTCGCTACC CGCTGCTGAA 6901 CCCTTTTCCG ACGCCGCTCA CCTGCTATCC GCTCTTCCCC AGGGCGCTAC AGCCGGAGGA 
6961 GCCCAAGCCC CAGCACAGCT ACATCGGCCT CATCGCCATG GCTATTCTAA GCTCTCCCGA 7021 AGGAAAACTG GTCCTTTCGG ACATCTATCA GCACATCCTC GACCACTATC CTTACTTCAG 7081 GACCCGGGGA CCGGGTTGGA GAAACTCCAT CAGGCATAAT TTGTCTCTCA ATGATTGTTT 7141 CATCAAGGCG GGAAGAAGCG CCAACGGAAA GGGACATTAC TGGGCAATTC ATCCCGCAAA 7201 TGTGGACGAC TTTAGAAAAG GGGACTTCAG GAGGAGGAAG GCACAAAGAA AGGTGAGGAA 7261 GCACATGGGG CTTGCCGTCG ATGAAGATGG GGCTGATTCG CCAAGTCCGC CGCCCTTGTC 7321 TGTGAGTCCG CCTGTCGTGC CAGGGCCTTC CACGTCCGTT TATCACACAG TGCCGGCTCG 7381 AGGTCCGTCT CGCAAGCGGC AGTTCGACGT GGCGTCGCTT TTGGCGCCGG ATTCCGGTGA 7441 AGACACCAAC GAAGAGGACA TCGACGTCGT CTCCAGTGAC CAACACCAAG AGACTTCACC 7501 CAAACAGTGG CCTAATATGT TTCCCATCGT TAATTATTAT CAAGCATTGT TACAAGCGAG 7561 GCCAGGAACG ACTGCAGAAA CTGCCACCGA CACCACAGAC TCTTAAccta ggatggcggc 7621 cgcctgcagc tggcgccatc gatacgcgta cgtcgcgacc gcggacatgt acagagctcg 7681 agatgatcgg tttttgttcc tacttagtta attgtgatag tttatttttg taaataatag 7741 tacgtaaagt tactttagtt ataataaagt agatagtgac tgcattaagt ttaaagactg 7801 atttttgtag attatttaaa aatttttaat aaattattta atttaaagat aaaaagttgt 7861 ttcattttaa aataataaaa aacttaccgc attagtcgtg gccttggcag tattaagcgc 7921 aaacgggctc tccttcgtat gcccatcagg aatatagaaa aaaacaactg gctgctcact 7981 agtggccacg tgggccgtgc accttaagct tggcgcgccg tacgcgatcg taagcttgat 8041 gagtttggac aaaccacaac tagaatgcag tgaaaaaaat gctttatttg tgaaatttgt 8101 gatgctattg ctttatttgt aaccattata agctgcaata aacaagttaa caacaacaat 8161 tgcattcatt ttatgtttca ggttcagggg gaagtgtggg aggtttttta aagcaagtaa 8221 aacctctaca aatgtggtat ggctgattat gatctagagt cgcggccgcc ttaacgaaat 8281 aattaattgt ttccattcga cacgatattg gtgctatttg ccgaccccca attgcacaaa 8341 tgacttctca tagacgtaga caaggggggg atgtaggacc gtggtatcaa aaacgtcgat 8401 aaattgaaaa ggtccacgaa aactttatac cgatcgctga aaaacaaaat caaacattgc 8461 aaaaaccgtg ttgaaatcaa tttcacctta acgttgacct taaatactga taccccgaag 8521 agccccctgt tcctaactgc gaagaaccaa tcattctttg caccatcaaa acatgattat 8581 ctgaaattat aaaatttaaa caaaaacaat tgacgtacgg atttactaac accgccattt 8641 cgtgatcagc gagtctatgt ccatcaaaag tgttagaatt tggtggggct gactgaacct 8701 gggttcgtcc ctgtaaaagg tgatcatgat agccccttgc agggctttgt gtgagaagcg 8761 cctctcgccg cgcgacatta gagcctcatg gacctcaact ttaaaaatcg actcgtaaac 8821 ctcgcgtctc ttctccaaat cgttcaattt gtaacgcttt aaagtctccg cctcctcctt 8881 ctccgccagc tctttttgct cggttaaaag cttctcgacc gctttttggt acttccccca 8941 gaaattaaac ccctcgagct ccagccccgg cgttcgctcc agccaccgct ggaccaaatc 9001 ggtcaaagac ggctctttct ccgacttggc gatttgctcg agcgcttttt cgtcatttcc 9061 gaacactttc gagtagttct ggttgtattt gacgcggttt tcctggcgca ccccgagctt 9121 gttttccagg agcctgaact gcaggctctg gaacccggaa gcgggccgca gataacaccg 9181 gaagtccatg aaatcgagcg gtgtcatcgt ttccagaatc atcacttggt ccaccaagac 9241 ctacaattgc gacagtttta tctcgtgacc gcaatttgtt gcgaaattgt gaaccttgag 9301 gatcaaaaca acacgattga ggcgtttgag gatttccaaa gtttgcgact cttctaaaac 9361 gtcgctgaaa atgttgcgga tcgagtccag ctcgtagatt atttgtttga accataactc 9421 atacgctgga attacttgtt atagttaatt acggttaagt gggtttttta ccttgatggg 9481 tcacaataaa taaatgctcg tcgtgcacgg gctggttatt ttgctcgctg agtaatcttt 9541 gggcttctaa aatcttgtcc aacatcaagt attcaccgta aagcatccca cattcttcgc 9601 tcagttggtc gccttcttgg gcttcactta atttacgatt aattgtaaat ggaataatta 9661 aattaaatca tacttacgag ggtctcagtg ggcaactcat tacgaccgcg gtaccgtcga 9721 cgttctagcg gtaccccgat tgtttagctt gttcagctgc gcttgtttat ttgcttagct 9781 ttcgcttagc gacgtgttca ctttgcttgt ttgaattgaa ttgtcgctcc gtagacgaag 9841 cgcctctatt tatactccgg cggtcgaggg ttcgaaatcg ataagcttgg atcctaattg 9901 aattagctct aattgaatta gtctctaatg aataatgatc cccgggcgag ctcgaattaa // 9961 ttcaac 
3xP3ITATA 10895...10807

Tc'vermilion 10668...9230

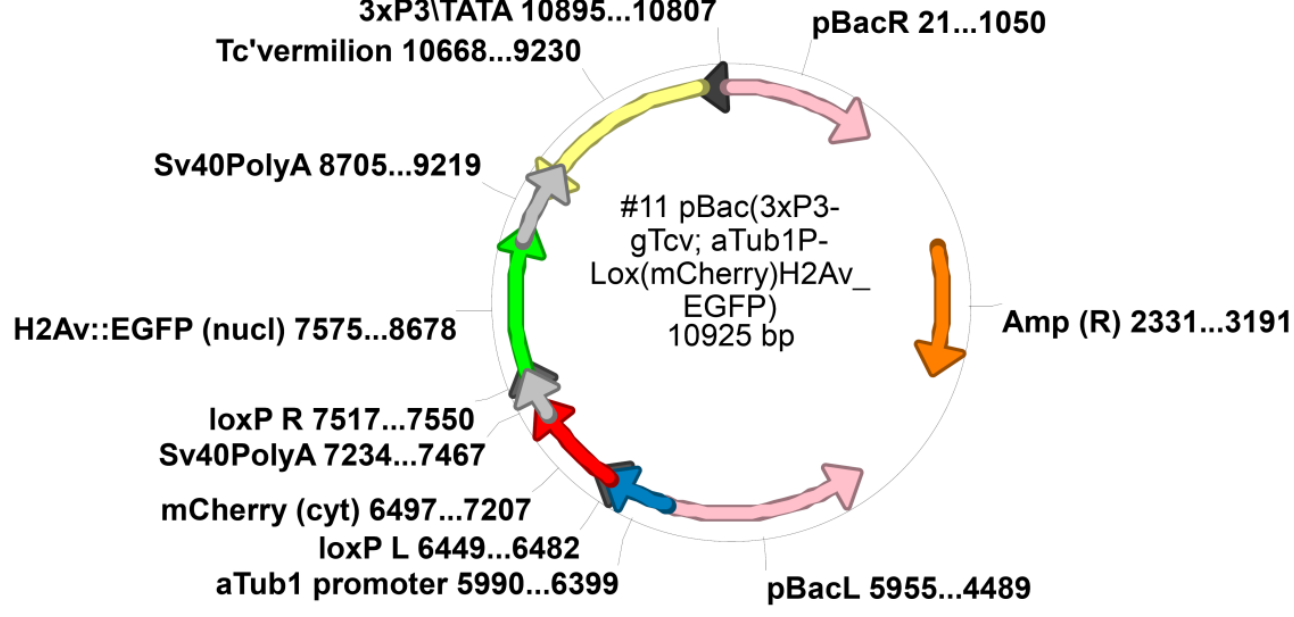

Figure S7.27 pB[3xP3-gTc'v;Tc' $\alpha$ Tub1P-IoxP(mcherry)-Tc'H2Av::EGFP].

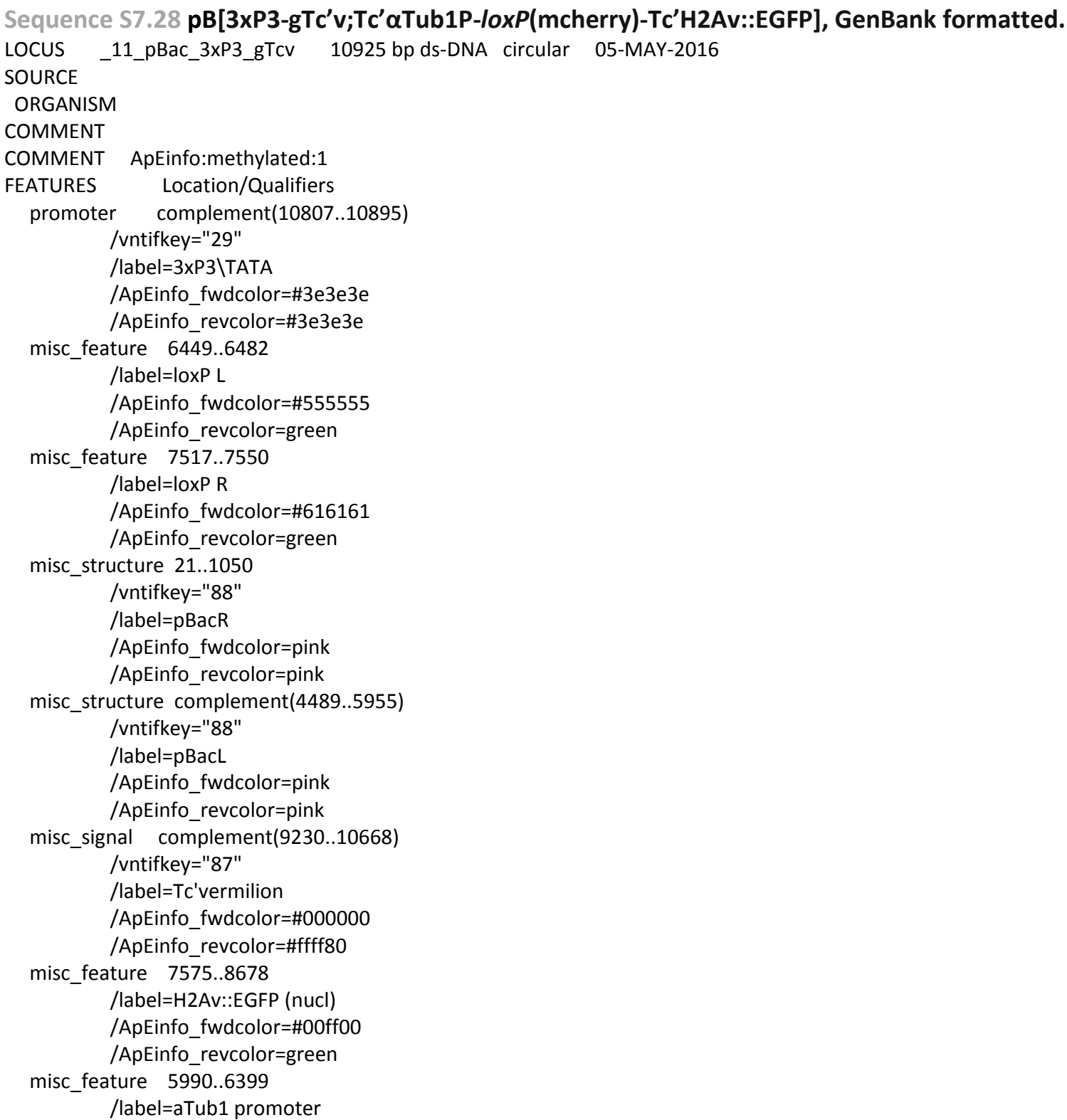

Sequence S7.28 pB[3xP3-gTc'v;Tc' $\alpha$ Tub1P-loxP(mcherry)-Tc'H2Av::EGFP], GenBank formatted.

ORGANISM

COMMENT

COMMENT ApEinfo:methylated:1

promoter complement(10807..10895)

vntifkey="29"

6449..6482

/ApEinfo_fwdcolor=\#555555

Evereolor

/ApEinfo fwdcolor=\#61616

/vntifkey="88"

/label=pBacR

/ApEinfo_fwdcolor=pink

ApEinfo revcolor=pink

/vntifkey="88"

/ApEinfo fwdcolor=pink

/vntifkey="87"

bel=Tc'vermilion

ture 7575..8678

/ApEinfo_fwdcolor=\#00ff00

ture 5990..6399

label=aTub1 promoter 


\author{
/ApEinfo_fwdcolor $=\# 0080 c 0$ \\ /ApEinfo_revcolor=green \\ misc_feature 6497..7207 \\ /label=mCherry (cyt) \\ /ApEinfo_fwdcolor=\#ff0000 \\ /ApEinfo_revcolor=green \\ misc_feature 2331..3191 \\ /label=Amp (R) \\ /ApEinfo_fwdcolor=\#ff8000 \\ /ApEinfo_revcolor=green \\ misc_feature $8705 . .9219$ \\ /label=Sv40PolyA \\ /ApEinfo_fwdcolor $=\# c 0 c 0 c 0$ \\ /ApEinfo_revcolor=green \\ misc_feature $7234 . .7467$ \\ /label=Sv4OPolyA(1) \\ /ApEinfo_label=Sv40PolyA \\ /ApEinfo_fwdcolor=\#c0c0c0 \\ /ApEinfo_revcolor=green
}

ORIGIN

1 cattgtggga accgtgcgat caaacaaacg cgagataccg gaagtactga aaaacagtcg 61 ctccaggcca gtgggaacat cgatgttttg ttttgacgga ccccttactc tcgtctcata 121 taaaccgaag ccagctaaga tggtatactt attatcatct tgtgatgagg atgcttctat 181 caacgaaagt accggtaaac cgcaaatggt tatgtattat aatcaaacta aaggcggagt 241 ggacacgcta gaccaaatgt gttctgtgat gacctgcagt aggaagacga ataggtggcc 301 tatggcatta ttgtacggaa tgataaacat tgcctgcata aattctttta ttatatacag 361 ccataatgtc agtagcaagg gagaaaaggt tcaaagtcgc aaaaaattta tgagaaacct 421 ttacatgagc ctgacgtcat cgtttatgcg taagcgttta gaagctccta ctttgaagag 481 atatttgcgc gataatatct ctaatatttt gccaaatgaa gtgcctggta catcagatga 541 cagtactgaa gagccagtaa tgaaaaaacg tacttactgt acttactgcc cctctaaaat 601 aaggcgaaag gcaaatgcat cgtgcaaaaa atgcaaaaaa gttatttgtc gagagcataa 661 tattgatatg tgccaaagtt gtttctgact gactaataag tataatttgt ttctattatg 721 tataagttaa gctaattact tattttataa tacaacatga ctgtttttaa agtacaaaat 781 aagtttattt ttgtaaaaga gagaatgttt aaaagttttg ttactttata gaagaaattt 841 tgagtttttg ttttttttta ataaataaat aaacataaat aaattgtttg ttgaatttat 901 tattagtatg taagtgtaaa tataataaaa cttaatatct attcaaatta ataaataaac 961 ctcgatatac agaccgataa aacacatgcg tcaattttac gcatgattat ctttaacgta 1021 cgtcacaata tgattatctt tctagggtta aataatagtt tctaattttt ttattattca 1081 gcctgctgtc gtgaataccg tatatctcaa cgctgtctgt gagattgtcg tattctagcc $1141 \mathrm{tttttagttt} \mathrm{ttcgctcatc} \mathrm{gacttgatat} \mathrm{tgtccgacac} \mathrm{attttcgtcg} \mathrm{atttgcgttt}$ 1201 tgatcaaaga cttgagcaga gacacgttaa tcaactgttc aaattgatcc atattaacga 1261 tatcaacccg atgcgtatat ggtgcgtaaa atatattttt taaccctctt atactttgca 1321 ctctgcgtta atacgcgttc gtgtacagac gtaatcatgt tttctttttt ggataaaact 1381 cctactgagt ttgacctcat attagaccct cacaagttgc aaaacgtggc attttttacc 1441 aatgaagaat ttaaagttat tttaaaaaat ttcatcacag atttaaagaa gaaccaaaaa 1501 ttaaattatt taatcgacca gttaatcaac gtgtacacag agcgcaaaaa acacgcagcc 1561 cgacgtgttg gctaaaatta ttaaatcaac ttgtgttata gtcacgattt gccgtccaac 1621 gtgttcctca aaaagttgaa gaccaacaag tttacggaca ctagttaatt atttgatttt 1681 gccccacttc attttgtggg atcacaattt tgttatattt taaacaaagc ttggcactgg 1741 ccgtcgtttt acaacgtcgt gactgggaaa accctggcgt tacccaactt aatcgccttg 1801 cagcacatcc ccctttcgcc agctggcgta atagcgaaga ggcccgcacc gatcgccctt 1861 cccaacagtt gcgcagcctg aatggcgaat ggcgcctgat gcggtatttt ctccttacgc 1921 atctgtgcgg tatttcacac cgcatatggt gcactctcag tacaatctgc tctgatgccg 1981 catagttaag ccagccccga cacccgccaa cacccgctga cgcgccctga cgggcttgtc 2041 tgctcccggc atccgcttac agacaagctg tgaccgtctc cgggagctgc atgtgtcaga 2101 ggttttcacc gtcatcaccg aaacgcgcga gacgaaaggg cctcgtgata cgcctatttt 2161 tataggttaa tgtcatgata ataatggttt cttagacgtc aggtggcact tttcggggaa 2221 atgtgcgcgg aacccctatt tgtttatttt tctaaataca ttcaaatatg tatccgctca 2281 tgagacaata accctgataa atgcttcaat aatattgaaa aaggaagagt atgagtattc 2341 aacatttccg tgtcgccctt attccctttt ttgcggcatt ttgccttcct gtttttgctc 2401 acccagaaac gctggtgaaa gtaaaagatg ctgaagatca gttgggtgca cgagtgggtt 2461 acatcgaact ggatctcaac agcggtaaga tccttgagag ttttcgcccc gaagaacgtt 2521 ttccaatgat gagcactttt aaagttctgc tatgtggcgc ggtattatcc cgtattgacg 
2581 ccgggcaaga gcaactcggt cgccgcatac actattctca gaatgacttg gttgagtact 2641 caccagtcac agaaaagcat cttacggatg gcatgacagt aagagaatta tgcagtgctg 2701 ccataaccat gagtgataac actgcggcca acttacttct gacaacgatc ggaggaccga 2761 aggagctaac cgcttttttg cacaacatgg gggatcatgt aactcgcctt gatcgttggg 2821 aaccggagct gaatgaagcc ataccaaacg acgagcgtga caccacgatg cctgtagcaa 2881 tggcaacaac gttgcgcaaa ctattaactg gcgaactact tactctagct tcccggcaac 2941 aattaataga ctggatggag gcggataaag ttgcaggacc acttctgcgc tcggcccttc 3001 cggctggctg gtttattgct gataaatctg gagccggtga gcgtgggtct cgcggtatca 3061 ttgcagcact ggggccagat ggtaagccct cccgtatcgt agttatctac acgacgggga 3121 gtcaggcaac tatggatgaa cgaaatagac agatcgctga gataggtgcc tcactgatta 3181 agcattggta actgtcagac caagtttact catatatact ttagattgat ttaaaacttc 3241 atttttaatt taaaaggatc taggtgaaga tcctttttga taatctcatg accaaaatcc 3301 cttaacgtga gttttcgttc cactgagcgt cagaccccgt agaaaagatc aaaggatctt 3361 cttgagatcc tttttttctg cgcgtaatct gctgcttgca aacaaaaaaa ccaccgctac 3421 cagcggtggt ttgtttgccg gatcaagagc taccaactct ttttccgaag gtaactggct 3481 tcagcagagc gcagatacca aatactgtcc ttctagtgta gccgtagtta ggccaccact 3541 tcaagaactc tgtagcaccg cctacatacc tcgctctgct aatcctgtta ccagtggctg 3601 ctgccagtgg cgataagtcg tgtcttaccg ggttggactc aagacgatag ttaccggata 3661 aggcgcagcg gtcgggctga acggggggtt cgtgcacaca gcccagcttg gagcgaacga 3721 cctacaccga actgagatac ctacagcgtg agcattgaga aagcgccacg cttcccgaag 3781 ggagaaaggc ggacaggtat ccggtaagcg gcagggtcgg aacaggagag cgcacgaggg 3841 agcttccagg gggaaacgcc tggtatcttt atagtcctgt cgggtttcgc cacctctgac 3901 ttgagcgtcg atttttgtga tgctcgtcag gggggcggag cctatggaaa aacgccagca 3961 acgcggcctt tttacggttc ctggcctttt gctggccttt tgctcacatg ttctttcctg 4021 cgttatcccc tgattctgtg gataaccgta ttaccgcctt tgagtgagct gataccgctc 4081 gccgcagccg aacgaccgag cgcagcgagt cagtgagcga ggaagcggaa gagcgcccaa 4141 tacgcaaacc gcctctcccc gcgcgttggc cgattcatta atgcagctgg cacgacaggt 4201 ttcccgactg gaaagcgggc agtgagcgca acgcaattaa tgtgagttag ctcactcatt 4261 aggcacccca ggctttacac tttatgcttc cggctcgtat gttgtgtgga attgtgagcg 4321 gataacaatt tcacacagga aacagctatg accatgatta cgaattcgag ctcggtaccc 4381 ggggatcctc tagagtcgac gctcgcgcga cttggtttgc cattctttag cgcgcgtcgc 4441 gtcacacagc ttggccacaa tgtggttttt gtcaaacgaa gattctatga cgtgtttaaa 4501 gtttaggtcg agtaaagcgc aaatcttttt taaccctaga aagatagtct gcgtaaaatt 4561 gacgcatgca ttcttgaaat attgctctct ctttctaaat agcgcgaatc cgtcgctgtg 4621 catttaggac atctcagtcg ccgcttggag ctcccgtgag gcgtgcttgt caatgcggta 4681 agtgtcactg attttgaact ataacgaccg cgtgagtcaa aatgacgcat gattatcttt 4741 tacgtgactt ttaagattta actcatacga taattatatt gttatttcat gttctactta 4801 cgtgataact tattatatat atattttctt gttatagata tcgtgactaa tatataataa 4861 aatgggtagt tctttagacg atgagcatat cctctctgct cttctgcaaa gcgatgacga 4921 gcttgttggt gaggattctg acagtgaaat atcagatcac gtaagtgaag atgacgtcca 4981 gagcgataca gaagaagcgt ttatagatga ggtacatgaa gtgcagccaa cgtcaagcgg 5041 tagtgaaata ttagacgaac aaaatgttat tgaacaacca ggttcttcat tggcttctaa 5101 cagaatcttg accttgccac agaggactat tagaggtaag aataaacatt gttggtcaac 5161 ttcaaagtcc acgaggcgta gccgagtctc tgcactgaac attgtcagat ctcaaagagg 5221 tccgacgcgt atgtgccgca atatatatga cccactttta tgcttcaaac tattttttac 5281 tgatgagata atttcggaaa ttgtaaaatg gacaaatgct gagatatcat tgaaacgtcg 5341 ggaatctatg acaggtgcta catttcgtga cacgaatgaa gatgaaatct atgctttctt 5401 tggtattctg gtaatgacag cagtgagaaa agataaccac atgtccacag atgacctctt 5461 tgatcgatct ttgtcaatgg tgtacgtctc tgtaatgagt cgtgatcgtt ttgatttttt 5521 gatacgatgt cttagaatgg atgacaaaag tatacggccc acacttcgag aaaacgatgt 5581 atttactcct gttagaaaaa tatgggatct ctttatccat cagtgcatac aaaattacac 5641 tccaggggct catttgacca tagatgaaca gttacttggt tttagaggac ggtgtccgtt 5701 taggatgtat atcccaaaca agccaagtaa gtatggaata aaaatcctca tgatgtgtga 5761 cagtggtacg aagtatatga taaatggaat gccttatttg ggaagaggaa cacagaccaa 5821 cggagtacca ctcggtgaat actacgtgaa ggagttatca aagcctgtgc acggtagttg 5881 tcgtaatatt acgtgtgaca attggttcac ctcaatccct ttggcaaaaa acttactaca 5941 agaaccgtat aagttcgaga tcggccggcc taggcgcgcc aagcttaagG CAACGACCGG 6001 GACCTCAGCT GTATAAAAAA TCTGAAAATA ATTGATAATG GTCGAAGGCT ACAAGGAAAA 6061 ATAATTCGAA AATTAAAGGC AAAGTGTGAA AATGCTGGAA ATAAATCGGG AAACACCGAA 6121 TTTTTGGTCA CAGGAACCAA CGAATTTTTT TTTGAATATT GATTTGAAAA GAGATCGAGA 6181 ACAAGGATAA CAATCTTCCG TTAGAGACCG TTGGTACCGA CATGGCGGGG AGCCAATCAG 6241 AAGTCTGGAA ATCGGGCGTT GGTTGCTGAC GTCAACAAGC TTATAAATAG CAACGACAGT 6301 TGAAAATCGA ATCAAAGTCG TTTGGAAAAA GCCAGAGCTT GTATTCCGAA GCGTACTCCC 
6361 GTTTTTCTGC TCTTTTGTGG GGTAATTTGT AAAACTCAAa ctagtcataa cttcgtatag 6421 gatactttat acgaagttat aactcgagat aacttcgtat agcatacatt atacgaagtt 6481 ataccggcag gagctcATGG TGAGCAAGGG CGAGGAGGAT AACATGGCCA TCATCAAGGA 6541 GTTCATGCGC TTCAAGGTGC ACATGGAGGG CTCCGTGAAC GGCCACGAGT TCGAGATCGA 6601 GGGCGAGGGC GAGGGCCGCC CCTACGAGGG CACCCAGACC GCCAAGCTGA AGGTGACCAA 6661 GGGTGGCCCC CTGCCCTTCG CCTGGGACAT CCTGTCCCCT CAGTTCATGT ACGGCTCCAA 6721 GGCCTACGTG AAGCACCCCG CCGACATCCC CGACTACTTG AAGCTGTCCT TCCCCGAGGG 6781 CTTCAAGTGG GAGCGCGTGA TGAACTTCGA GGACGGCGGC GTGGTGACCG TGACCCAGGA 6841 CTCCTCCCTG CAGGACGGCG AGTTCATCTA CAAGGTGAAG CTGCGCGGCA CCAACTTCCC 6901 CTCCGACGGC CCCGTAATGC AGAAGAAGAC CATGGGCTGG GAGGCCTCCT CCGAGCGGAT 6961 GTACCCCGAG GACGGCGCCC TGAAGGGCGA GATCAAGCAG AGGCTGAAGC TGAAGGACGG 7021 CGGCCACTAC GACGCTGAGG TCAAGACCAC CTACAAGGCC AAGAAGCCCG TGCAGCTGCC 7081 CGGCGCCTAC AACGTCAACA TCAAGTTGGA CATCACCTCC CACAACGAGG ACTACACCAT 7141 CGTGGAACAG TACGAACGCG CCGAGGGCCG CCACTCCACC GGCGGCATGG ACGAGCTGTA 7201 CAAGTAGgcg gccgcgtacg cgatcgtaag cttgaagata cattgatgag tttggacaaa 7261 ccacaactag aatgcagtga aaaaaatgct ttatttgtga aatttgtgat gctattgctt 7321 tatttgtaac cattataagc tgcaataaac aagttaacaa caacaattgc attcatttta 7381 tgtttcaggt tcagggggag gtgtgggagg ttttttaaag caagtaaaac ctctacaaat 7441 gtggtatggc tgattatgat ctagagtcat atgcataact tcgtatagga tactttatac 7501 gaagttataa ctcgagataa cttcgtatag catacattat acgaagttat accggcaggg 7561 taccCTAAAG AAAAATGGCT GGTGGCAAAG CAGGGAAAGA TTCGGGCAAA GCTAAAGCCA 7621 AGGCGGTGTC GCGCTCGGCG CGTGCCGGAC TCCAGTTCCC CGTGGGGAGG ATCCACCGCC 7681 ACTTGAAGAA TCGCACTACG AGCCATGGAC GCGTGGGGGC CACCGCGGCT GTGTATTCCG 7741 CCGCGATTTT GGAGTATTTG ACAGCTGAGG TCCTGGAGTT GGCCGGGAAC GCCAGTAAGG 7801 ACTTGAAGGT GAAGAGGATC ACCCCCAGGC ACTTGCAGCT GGCCATCAGA GGGGATGAGG 7861 AGCTGGACAG CCTGATCAAG GCCACTATCG CCGGCGGAGG CGTCATTCCA CACATCCACA 7921 AGAGTTTGAT CGGGAAAAAG GGCCACTCAC AGCCCGTCAT GGTGAGCAAG GGCGAGGAGC 7981 TGTTCACCGG GGTGGTGCCC ATCCTGGTCG AGCTGGACGG CGACGTAAAC GGCCACAAGT 8041 TCAGCGTGTC CGGCGAGGGC GAGGGCGATG CCACCTACGG CAAGCTGACC CTGAAGTTCA 8101 TCTGCACCAC CGGCAAGCTG CCCGTGCCCT GGCCCACCCT CGTGACCACC CTGACCTACG 8161 GCGTGCAGTG CTTCAGCCGC TACCCCGACC ACATGAAGCA GCACGACTTC TTCAAGTCCG 8221 CCATGCCCGA AGGCTACGTC CAGGAGCGCA CCATCTTCTT CAAGGACGAC GGCAACTACA 8281 AGACCCGCGC CGAGGTGAAG TTCGAGGGCG ACACCCTGGT GAACCGCATC GAGCTGAAGG 8341 GCATCGACTT CAAGGAGGAC GGCAACATCC TGGGGCACAA GCTGGAGTAC AACTACAACA 8401 GCCACAACGT CTATATCATG GCCGACAAGC AGAAGAACGG CATCAAGGTG AACTTCAAGA 8461 TCCGCCACAA CATCGAGGAC GGCAGCGTGC AGCTCGCCGA CCACTACCAG CAGAACACCC 8521 CCATCGGCGA CGGCCCCGTG CTGCTGCCCG ACAACCACTA CCTGAGCACC CAGTCCGCCC 8581 TGAGCAAAGA CCCCAACGAG AAGCGCGATC ACATGGTCCT GCTGGAGTTC GTGACCGCCG 8641 CCGGGATCAC TCTCGGCATG GACGAGCTGT ACAAGTAAgg cgcgccgtac gcgatcgtaa 8701 gcttgatgag tttggacaaa ccacaactag aatgcagtga aaaaaatgct ttatttgtga 8761 aatttgtgat gctattgctt tatttgtaac cattataagc tgcaataaac aagttaacaa 8821 caacaattgc attcatttta tgtttcaggt tcagggggaa gtgtgggagg ttttttaaag 8881 caagtaaaac ctctacaaat gtggtatggc tgattatgat ctagagtaga tctgtcgacg 8941 tcccatggcc attcgaattc ggccggccta ggcgcgccgt acgcgatcgt aagcttgatg 9001 agtttggaca aaccacaact agaatgcagt gaaaaaaatg ctttatttgt gaaatttgtg 9061 atgctattgc tttatttgta accattataa gctgcaataa acaagttaac aacaacaatt 9121 gcattcattt tatgtttcag gttcaggggg aagtgtggga ggttttttaa agcaagtaaa 9181 acctctacaa atgtggtatg gctgattatg atctagagtc gcggccgcct taacgaaata 9241 attaattgtt tccattcgac acgatattgg tgctatttgc cgacccccaa ttgcacaaat 9301 gacttctcat agacgtagac aaggggggga tgtaggaccg tggtatcaaa aacgtcgata 9361 aattgaaaag gtccacgaaa actttatacc gatcgctgaa aaacaaaatc aaacattgca 9421 aaaaccgtgt tgaaatcaat ttcaccttaa cgttgacctt aaatactgat accccgaaga 9481 gccccctgtt cctaactgcg aagaaccaat cattctttgc accatcaaaa catgattatc 9541 tgaaattata aaatttaaac aaaaacaatt gacgtacgga tttactaaca ccgccatttc 9601 gtgatcagcg agtctatgtc catcaaaagt gttagaattt ggtggggctg actgaacctg 9661 ggttcgtccc tgtaaaaggt gatcatgata gccccttgca gggctttgtg tgagaagcgc 9721 ctctcgccgc gcgacattag agcctcatgg acctcaactt taaaaatcga ctcgtaaacc 9781 tcgcgtctct tctccaaatc gttcaatttg taacgcttta aagtctccgc ctcctccttc 9841 tccgccagct ctttttgctc ggttaaaagc ttctcgaccg ctttttggta cttcccccag 9901 aaattaaacc cctcgagctc cagccccggc gttcgctcca gccaccgctg gaccaaatcg 9961 gtcaaagacg gctctttctc cgacttggcg atttgctcga gcgctttttc gtcatttccg 10021 aacactttcg agtagttctg gttgtatttg acgcggtttt cctggcgcac cccgagcttg 10081 ttttccagga gcctgaactg caggctctgg aacccggaag cgggccgcag ataacaccgg 
10141 aagtccatga aatcgagcgg tgtcatcgtt tccagaatca tcacttggtc caccaagacc 10201 tacaattgcg acagttttat ctcgtgaccg caatttgttg cgaaattgtg aaccttgagg 10261 atcaaaacaa cacgattgag gcgtttgagg atttccaaag tttgcgactc ttctaaaacg 10321 tcgctgaaaa tgttgcggat cgagtccagc tcgtagatta tttgtttgaa ccataactca 10381 tacgctggaa ttacttgtta tagttaatta cggttaagtg ggttttttac cttgatgggt 10441 cacaataaat aaatgctcgt cgtgcacggg ctggttattt tgctcgctga gtaatctttg 10501 ggcttctaaa atcttgtcca acatcaagta ttcaccgtaa agcatcccac attcttcgct 10561 cagttggtcg ccttcttggg cttcacttaa tttacgatta attgtaaatg gaataattaa 10621 attaaatcat acttacgagg gtctcagtgg gcaactcatt acgaccgcgg taccgtcgac 10681 gttctagcgg taccccgatt gtttagcttg ttcagctgcg cttgtttatt tgcttagctt 10741 tcgcttagcg acgtgttcac tttgcttgtt tgaattgaat tgtcgctccg tagacgaagc 10801 gcctctattt atactccggc ggtcgagggt tcgaaatcga taagcttgga tcctaattga 10861 attagctcta attgaattag tctctaatga ataatgatcc ccgggcgagc tcgaattaat 10921 tcaac

//

\section{Videofiles}

Videofile S7.29 Compacted LSFM video, ventral and dorsal view, maximum projections.

Videofile S7.30 Compacted LSFM video, lateral view, rendered 3D projection.

Videofile S7.31 Compacted LSM video, antero-frontal view, maximum projections.

Videofile S7.32 Compacted LSFM video, antero-frontal view, maximum projections 
8

\section{Curriculum Vitae}

\section{Personal Detalls}

$\begin{array}{ll}\text { NAME: } & \text { Peter Kitzmann } \\ \text { NATIONALITY: } & \text { German } \\ \text { DATE OF BIRTH: } & \mathbf{0 2 . 1 2 . 1 9 8 5} \\ \text { PLACE OF BIRTH: } & \text { Lübeck, Germany }\end{array}$

\section{AddRess of CURRent Place of Work}

UNIVERSITY: Georg-August-Universität Göttingen

INSTITUTE: Johann-Friedrich-Blumenbach-Institute of Zoology and Anthropology

DEPARTMENT: Department of Evolutionary Developmental Genetics

STREET: Justus-von-Liebig-Weg 11

Town: $\quad 37077$ Göttingen

COUNTRY: Germany

TelePHONE: + +49-551-3910124

E-MAIL: $\quad$ peter.kitzmann@stud.uni-goettingen.de

\section{UNIVERSITY STUDIES}

SINCE 08/12 Ph.D. Thesis "Morphogenesis and Genetic Regulation of the Insect Head" at the Göttingen Graduate School for Neurosciences and Molecular Biosciences (GGNB) in the group of Gregor Bucher, Department of Developmental Evolutionary Genetics, Georg-August-Universität Göttingen.

02/11-10/11 Master Thesis "Analysis of RNAi Phenotypes" in the group of Gregor Bucher, Department of Developmental Biology, Georg-August-Universität Göttingen 
10/09-10/11 M.Sc. Studies in the "Developmental, Neural and Behavioral Biology" program with emphasis on Cell and Developmental Biology, Georg-August-Universität Göttingen

04/09-08-09 Bachelor Thesis "Molekulare und phänotypische Analyse von Insertionsmutanten des rotbraunen Reismehlkäfers Tribolium castaneum" in the group of Gregor Bucher, Department of Developmental Biology, Georg-AugustUniversität Göttingen

10/06-08/09 B.Sc. Studies in the "Biology" program, Georg-August-Universität Göttingen

\section{SUPERVISION}

07/15-01/16 Master Thesis of Felix Kaufholz “CRISPR-mediated Transgene Knock-in to Disrupt and Investigate Function of FoxQ2 in the Beetle Tribolium castaneum"

08/14-11/14 Lab Rotation of Felix Kaufholz "Molecular Cloning of Two Fluorescent $\alpha$-Tubulin Fusions and Evaluation of Heat shock induced Cell Marking for Fate-mapping in Tribolium castaneum (Coleoptera)"

08/12-10/12 Lab Rotation of Julia Meyer "Improvement of the Visualizing Techniques to Study Insect Head Development in Tribolium castaneum"

INTERNSHIPS

01/11-03/11 Lab Rotation: "Characterization of the Annotated Lipase CG9186 in Drosophila melanogaster", in the group of Mathias Beller, Department of Molecular Developmental Biology, Max-Planck-Institute for Biophysical Chemistry.

09/10-11/10 Lab Rotation: "Molecular and Phenotypic Analysis of the Transgenic Line G11721 in Tribolium castaneum", in the group of Gregor Bucher, Department of Developmental Biology, Georg-August-Universität Göttingen.

\section{CONTRIBUTION TO CONFERENCES}

TALK

$03 / 13$

"RNAi Phenotypes are Influenced by the Genetic Background of the Injected Strain", iBeetle symposium "New Horizons in Molecular Zoology", Göttingen, Germany

09/12 "RNAi Phenotypes are Influenced by the Genetic Background of the Injected Strain", $9^{\text {th }}$ GfE Summer School, Günzburg, Germany 


\section{POSTER}

03/15 "Morphogenesis and Genetic Regulation of the Insect Head", Joint Meeting of the German and French Societies of Developmental Biologists, Nuremberg, Germany

07/14 "Morphogenesis and Genetic Regulation of the Insect Head", $5^{\text {th }}$ Meeting of the European Society for Evolutionary Developmental Biology, Vienna, Austria

12/13 "Morphogenesis and Genetic Regulation of the Insect Head", GGNB Science Day, Göttingen, Germany

\section{Publications}

Kitzmann P, Schwirz J, Schmitt-Engel C and Bucher G. 2013, RNAi phenotypes are influenced by the genetic background of the injected strain. BMC Genomics 2013, 14:5

Fu J, Posnien N, Bolognesi R, Fischer TD, Rayl P, Oberhofer G, Kitzmann P, Brown SJ, Bucher G. 2012, Asymmetrically expressed axin required for anterior development in Tribolium. PNAS 7782-7786 May 15, 2012 
